\section{NBS}

\section{PUBLICATIONS}

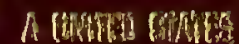

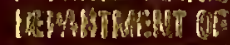

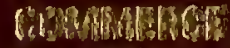

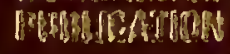

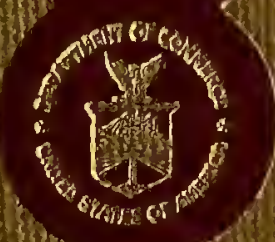

\section{AI1100 98976?}

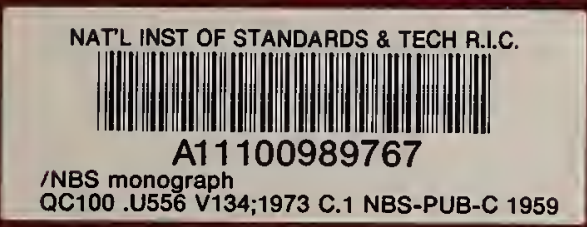

\section{MBS MONOGRAPH 134}

\title{
Space Groups and Lattice Complexes
}

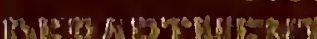

U.

Gorrartarea

$$
\text { Q }
$$

Nathongl Pureats of stanceros 

- 



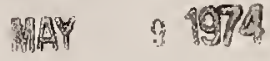 \\ 180724 \\ Space Groups and Lattice Complexes}

\section{Werner FISHER}

Mineralogisches Institut der Philipps-Universität

D-3550 Marburg/Lahn, German Federal Republic

\section{Hans BURZLAFF}

Lehrstuhl für Kristallographie der

Friedrich-Alexander-Universität Erlangen-Nürnberg

D-8520 Erlangen, German Federal Republic

\section{Erwin HELLNER}

Fachbereich Geowissenschaften der Philipps-Universität

D-3550 Marburg/Lahn, German Federal Republic

\section{J. D. H. DONNAY'}

Départment de Géologie, Université de Montréal

CP 6128 Montreal 101, Quebec, Canada

Formerly, The Johns Hopkins University, Baltimore, Maryland

Prepared under the auspices of the Commission on International Tables, International Union of Crystallography, and the Office of Standard

Reference Data, National Bureau of Standards.

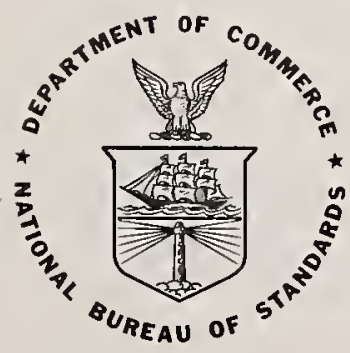

U.S. DEPARTMENT OF COMMERCE, Frederick B. Dent, Secretary NATIONAL BUREAU OF STANDARDS, Richard W. Roberts, Direcłor

Issued May 1973 
Library of Congress Catalog Number: 73-600099

\section{National Bureau of Standards Monograph 134}

Nat. Bur. Stand. (U.S.), Monogr. 134, 184 pages (May 1973)

CODEN : NBSMA6 
Abstract

The lattice complex is to the space group what the site set is to the point group - an assemblage of symmetry-related equivalent points. The symbolism introduced by Carl Hermann has been revised and extended. A total of 402 lattice complexes are derived from 67 Weissenberg complexes. The Tables list site sets and lattice complexes in standard and alternate representations. They answer the following questions: What are the co-ordinates of the points in a given lattice complex? In which space groups can a given lattice complex occur? What are the lattice complexes that can occur in a given space group? The higher the symmetry of the crystal structures is, the more useful the latticecomplex approach should be on the road to the ultimate goal of their classification.

Key words: Crystallography; crystal point groups; crystal structure; lattice complexes; site sets; space groups.

\section{Acknowledgments}

We wish to acknowledge support received from the PhilippsUniversität, The Johns Hopkins University, the National Bureau of Standards and the International Union of Crystallography.

Special thanks go to our students and assistants, both in Marburg and in Baltimore, for their enthusiastic participation in the construction and the checking of the tables.

The authors 
$\mathrm{T} 0$

T H E

M E M O R Y

O F

C A R L H E I N R I C H H E R M A N $(1898-1961)$

Dr.Phil. of the University of Göttingen, Professor of Crystallography at the University of Marburg-on-the-Lahn, Germany 
TEXT

Part I. SITE SETS

1. Generalities

1.1 Definitions

1.2 Rules of Splitting

1.3 Special Positions

2. Description of Site Sets

3. Occurrencess of Site Sets

4. Subgroup Relations

5. Site Sets and Their Occurrences in Oriented Subgroups 9

5.1 Cubic Degradation

5.2 Hexagonal Degradation

Part II. LATTICE COMPLEXES 11

6. Generalities

6.1 Definitions

6.2 The Lattice-Complex Symbol

6.21 Invariant Lattice Complexes

6.22 Variant Lattice Complexes

6.3 Rules for Selecting the Generating Lattice Complex 6.4 Distribution Symmetry

7. Weissenberg Complexes as Generating Complexes 18

7.1 The Weissenberg Complexes 18

7.2 Tables of Weissenberg Complexes 19

7.3 Figures of Weissenberg Complexes ig

8. List of the Lattice Complexes and Their 0ccurrences 20

g. List of Space Groups with Lattice-Complex Representations 23

Appendix. Subgroup Relations 23

References $\quad 26$

FIGURES

1-47 Site Sets in Stereographic Projection 29

48 Subgroup Relations in Point Groups 34

49-53 Cubic Lattice Complexes 35

54-57 Hexagonal Lattice Complexes 40

58-60 Tetragonal Lattice Complexes 44

61-63 Orthorhombic Lattice Complexes 47

64-65 Monoclinic Lattice Complexes 50

TABLES

1 Rules of Splitting 54

Site Sets:

2 Cubic

Hexagonal and Rhombohedral

55

Rhombohedral (in R co-ordinates) 58

Tetragonal $\quad 59$

Orthorhombic 60

Monoclinic 61

Anorthic (Triclinic) 61

g-15 Site Sets in Positions in Point Groups 62

$\begin{array}{lll}16 & \text { Cubic Degradation } & 64 \\ 17 & \text { Hexagonal Degradation } & 66\end{array}$

Weissenberg Complexes

18 Cubic

Hexagonal and Rhombohedral

Tetragonal

Orthorhombic

Monocl inic

Anorthic (Triclinic) 
Occurrence of Lattice Complexes

25 Cubic

Invariant $\quad 85$

Univariant $\quad 88$

$\begin{array}{ll}\text { 8ivariant } & 92\end{array}$

Trivariant 93

26-27 Hexagonal and Rhombohedral
Invariant

$\begin{array}{lr}\text { Invariant } & 95 \\ \text { Univariant } & 98\end{array}$

8ivariant 101

28 Trivariant 103

Invariant $\quad 105$

Univariant 109

8ivariant $\quad 114$

29 Orthorhombic

Invariant $\quad 119$

Univariant $\quad 121$

8ivariant $\quad 124$

Invariant $\quad 129$

Univariant $\quad 129$

8ivariant $\quad 130$

Trivariant 130

31 Anorthic (Triclinic) 131

Space Groups with Lattice-Complex Representations

Cubic

Hexagonal and Rhombohedral 138

Rhombohedral (in R co-ordinates) 148

$\begin{array}{ll}\text { Tetragonal } & 149\end{array}$

Orthorhombic 158

$\begin{array}{lll}37 & \text { Monoclinic } & 165 \\ 38 & \text { Anorthic (Triclinic) } & 167\end{array}$

Appendix

39-43 Subgroup Relations 169

$\begin{array}{ll}\text { Index } & 175\end{array}$

Errata 
A Zattice is defined, sensu stricto, as the assemblage of all the points that are the termini of the vectors

$$
\vec{L}(u v w)=u \vec{a}+v \vec{b}+w \vec{c},
$$

where $\vec{a}, \vec{b}, \vec{c}$ are three non-coplanar vectors and $u, v, w$ are integers that can take all possible values, positive, negative or zero. The lattice points are called nodes so they can be distinguished from other points in space. A lattice is the geometrical expression of a translation group: if a node is taken as origin, any otiner node can be obtained from it by means of a translation that is one of the vectors $\vec{L}(u v w)$.

As defined above, a lattice would always be primitive. In the case of symmetrical lattices, a multiple cell can be used to define the lattice; examples: cI, cubic body-centered; cF, cubic facecentered; etc. Centered lattice modes are useful in that they display the lattice symmetry, but they are no more than alternate descriptions of lattices that could be defined by primitive cells. Whatever description is chosen, the lattice nodes are the same, and, therefore, the translation group is also tine same.

After 1912 the word lattice was given various physical meanings, in addition to its original geometrical meaning, in the description of crystal structures. A structure in which all the atoms were of one kind, situated at the nodes of a lattice, could be described as a "lattice" of such atoms. For example the "lattice" of $\mathrm{Cu}$ atoms was used to designate the crystal structure of copper metal. Atoms of one kind were referred to as a "lattice" even in cases where they did not occupy the nodes of any lattice; as the carbon atoms in diamond, for instance, which -- to this day! -- are described as forming the "diamond lattice". In a crystal structure composed of more than one kind of atoms, CsCl for example, as many "atomic lattices" had to be distinguished as there were different kinds of atoms, whence "the cesium lattice" and "the chlorine lattice". The CsCl structure was thus described by means of "two interpenetrating lattices" instead of "two atoms repeated by a primitive cubic lattice". We realize nowadays how unfortunate this concept of interpenetration was, for there are as many interpentrating lattices as one cares to pick points in the cell, whether these points are occupied by atoms or not. As the crystal structures that were worked out increased in complexity, the word lattice came to be used for any set of equivalent atoms in a structure, whether the atoms were equivalent by lattice translations or by other symmetry operations in the space group. Even when the atomic sets did occupy the nodes of lattices (s.s.), different "lattices" were found to occur in the same structure; as in fluorite, for instance, where the "calcium lattice" is cF (cubic face-centered) with cell edge $a$, whereas the "fluorine lattice", meaning the "lattice" of fluorine atoms, is cP (cubic primitive) with cell edge $a / 2$. Confusion reached a climax when the word "lattice" was given another additional meaning to designate the collection of all the sets of atomic centers in the crystal structure, in other words, the crystal structure itself!

Paul Niggli (1919) introduced the term Zattice complex to designate a set of crystallograpinically equivalent atoms in a crystal structure. Examples: the $\mathrm{Na}$ atoms in $\mathrm{NaCl}$, the $\mathrm{C}$ atoms in diamond, the Cs atoms in $\mathrm{CsCl}$, etc. Consider the carbon atoms in diamond. They can be described in two ways: either eight "interpenetrating carbon lattices" or a cell content of 8 carbon atoms repeated by one lattice. 1 Niggli visualized the former, as was common at that time among German-speaking crystallographers. Not only are the carbon atoms of any one of the eight "lattices" equivalent (both crystallographically and chemically) by lattice translations, but the "carbon lattices" themselves are equivalent, either by lattice translation or by some other spacegroup-symmetry operation. The set of "atomic lattices" could thus be called a complex of equivalent lattices or a lattice complex for short. By the same token.a crystal form, being a set of equivalent faces, could be dubbed a face complex.

Niggli discusses the analogy between the crystal forms of crystal morphology and the lattice complexes

1 Note that this lattice is taken as primitive $(\mathrm{cP})$; with a face-centered lattice (cF), two atoms are repeated by the lattice. 
of crystal structure, pointing out that this analogy is not complete. He also snows the importance of 1attice complexes ${ }^{1}$ in three fields: (1) the description of structures; (2) the crystal-structure determination itself, in winich structure factors $F(h k l)$ should be computed by summing over lattice complexes rather than individual atoms; (3) the description of space groups.

Wiggli broaches the subject over and over again: in the several editions of his Lehrbuch der Uineralogie $(1920,1924,1941)$ and in his Gmondbegriffe (1928). Although he attempts to systematize the nomenclature, he does not have any easy symbolism for lattice complexes.

Carl Hermann recognized the importance of the lattice complexes and devised a method for symbolizing invariant and univariant lattice complexes, which appeared in the 1935 edition of the Intermational Tables (see Chapters VI and VII). Sucin a complex was designated by the symbol of the space group of niginest symetry that contains the lattice complex as a point position ${ }^{2}$ of inighest symmetry; the space-group symbol being followed by tine conventional Wyckoff letter of tnis point position. If more tinan one such position is available, the one that includes the origin (000) among its sites is the one to be chosen. This scheme is applicable to all lattice complexes, including the trivariant ones.

1 For practical applications of lattice complexes in 2 and 3 dimensions, see Burzlaff, Fischer and Hellner (1968 and 1969); Fischer (1968); Smimova (1966): Smirnova and Poteshnova (1966); Hellner (1965): Loeb (1967 and 1970).

2 Point position or, more simply, position is used as a collective noun to designate a set of equivalent points (either in a point group or in a space group). It corresponds to the German Punktlage and the French position. The term point position was used in the English text of the 1935 edition of the Intemational Taoles; it has priority over the periphrase "set of positions" used in the 1952 edition.
In 1953, in a paper given at the Erlangen meeting of the Crystallography Section of the German Mineralogical Society, Hermann outlined a symbolism for univariant lattice complexes, based on the splitting of the points of an invariant lattice complex in a number of directions depending on the available degrees of freedom. Hermann published this talk in 1960, adding new designations for the bivariant lattice complexes, the space-group symbol being slightly altered by the underlining of the appropriate $m$.

P. Niggli's original concept of lattice complex was somewhat modified by Hermann (1935) and by J.D.H. Donnay, E. Hellner and A. Niggli (1966). This last publication is a report on a Symposium held at the University of Kiel, February 17-28, 1964, at which the symbolism of lattice complexes was discussed by A. de Vries and $P$. Th. Beurskens (University of Utrecht), R. Allmann, H. Burzlaff, W. Fischer, U. Wattenberg, the late Gudrun Weiss, and the three rapporteurs.

The fundamental feature of a lattice complex; which was pointed out by Hermann in 1953, is that its points either are themselves invariant or are distributed in symmetrical clusters around the (invariant) points of an invariant complex. Each invariant point in its surroundings possesses its own point symmetry, so that the problem 1 argely reduces to symbolizing sets of equivalent points in a point group. Part I, accordingly, deals with site sets in point groups. If we draw a sphere around a point taken as the origin and let the symmetry elements of a point group go througin the origin, symmetry-related points will lie on the surface of the sphere. The poles of the faces of a crystal form constitute a familiar example of a set of equivalent points. The co-ordinates of one face pole are of the form $h a^{\star}, k b^{\star}$, $z c^{*}$, with $h, k, l$ integers; they are the co-ordinates of a node in the reciprocal lattice. In this work the co-ordinates will be taken in direct space: $x=u^{\prime} a$, $y=v^{\prime} b, z=w^{\prime} c$, where $u^{\prime}, v^{\prime}, w^{\prime}$ need not 'je integers.

Part II applies the results of part I to lattice complexes. 


\section{GENERALITIES}

1.1 Symbolism and definitions

The lattice, the infinite assemblage of points that geometrically expresses the triple periodicity of a crystal, is the basis for Bravais' definition of the crystal systems. It possesses, at any one of its nodes, a point symmetry which is one of the 32 crystal point groups. There exist seven such lattice symmetries, to which Bravais' seven crystal systems correspond. The point group of a lattice, together with the subgroups that constitute its merohedries, are all the point groups that are possible as crystal symmetries in the corresponding crystal system. When the crystal symmetry is the same as the lattice symmetry, it is called holohedral symmetry or holohedry. Examples: $\overline{3} 2 / \mathrm{m}$ is the rhombohedral-1attice symmetry; in the rhombohedral system it is the holohedry; $3 m$, 32,5 and 3 are its merohedries; any substance with a rhombohedral lattice can only have, as crystal symmetry, one of tirese five trigonal symmetries. The hexagonal-lattice symmetry, on the other hand, is $6 / m 2 / m 2 / m$; it has eleven merohedries (down to its ogdonedry 3); any substance with a hexagonal lattice can have, as crystal symmetry, any one of these twelve point groups, which include the five trigonal symmetries.

The Hermann-Mauguin symbolism rests on the fundamental concept of the symmetry directions in the lattice. A symmetry direction is defined as a symmetry axis of proper or improper rotation; in other words, a rotation axis ${ }^{1}$ or the normal to a mirror. Equivalent symmetry directions that pass through the node taken as the origin of a lattice, are symbolized in a column of the Hermann-Mauguin symbol: an Arabic numeral indicates a symmetry axis along the direction under consideration, the letter $m$ represents a mirror perpendicular to it. The number of columns in the Hermann-Mauguin symbol is the number of kinds of equi-

1 An altemating axis lof rotatory inversion or rotatory reflection) is a symmetry direction since it contains a rotation axis as a subgroup. Examples: $\overline{4}$ contains $2 ; \overline{6}$ and $\overline{3}$ both include 3 . Note that $\overline{2}$ is the normal to a mirror. valent symmetry directions. Example: in the hexagonal system, the three columns of the symbol refer to: (1) the principal direction (or primary axis); (2) the lateral axes of the 1st kind (Neben axes or secondary axes); and (3) the lateral axes of the 2nd kind (zwischen axes or tertiary axes). The most symmetrical point group is the holohedry, $6 / \mathrm{m} 2 / \mathrm{m} 2 / \mathrm{m}$ or $6 /$ mom for short. The symmetry directions of the lattice are what Carl Hermann called the Blickrichtungenthe directions in which you look to see the symmetry.

Note that, in the five trigonal classes, the Hermann-Mauguin symbol can easily be made to distinguish between the case of a rhombohedral lattice and that of an hexagonal one (Donnay, 1969): two-column symbols (Table 11) include 31 and 31 ; three-column symbols (Table 10) are explicited as $\overline{3} 11$ and 311 . In every case the figure 1 stands for symmetry directions of the lattice that are not symmetry directions for the crystal as a whole.

A crystallographic site set (here called simply site set for short) is defined as a set of $N$ equivalent points that are symmetry related in at least one of the 32 crystallographic point groups and can, therefore, occupy a position 1 in this point group. A site set will be called an N-pointer (after the German $\mathrm{N}$-Punktner or $\mathrm{N}$-Punkter). Every site of the $\mathrm{N}$-pointer is invariant under certain symmetry operations, which constitute a subgroup of order $N$ of the crystallographic point group. This subgroup is called point symmetry of the site or site symmetry of the point group. A site thus lies on one, or more than one, symmetry element, except in the trivial case where the site symmetry reduces to identity. (Symmetry elements are mirrors, axes and the center.) The actual symmetry elements in the various sites of a site set differ from site to site: although equivalent mirrors and equivalent axes are found in every site, they are in general differently oriented from one site to another. Only one point can have the center as an element of the site symmetry -- it is the fixed point in the point group, taken as the origin 000. P. Niggli calls it Hauptsymmetriepunkt (HSP), which we propose to translate by highest symmetry point.

I see Introduction, last footnote. 
Examples. A site set is provided by the corners of the cube. The eight points have the following coordinates in the usual Cartesian system, with the origin in the center:

$x \bar{x}, \bar{x} \bar{x}, \bar{x} x \bar{x}, \bar{x} \bar{x} x, \bar{x} \bar{x} \bar{x}, \bar{x} x x, x \bar{x} x, x \bar{x} \bar{x}$.

They constitute a set of equivalent sites in any one of three point groups: $m 3 m$ (of order 48), 432 (of order 24) and $m^{3}$ (of order 24), with site symmetry $3 m$ (of order 6) in $m 3 m$ and 3 (of order 3 ) in both 432 and $m 3$. In all three point groups, each site thus lies on a 3 -fold rotation axis, which coincides with the line of intersection of three mirrors in the holohedral point group $m 3 m$. In every case the site symmetry is a subgroup of index 8 of the appropriate point group.

Another example is the assemblage of the 48 points whose co-ordinates are obtained by replacing $x x x$, in the first example, by each of the six complete permutations of xyz. These points are equivalent sites in point group $m 3 m$, and their site symmetry is 1 , the trivial subgroup of index 48 .

In a point group, as a limiting case resulting from a specialization of site co-ordinates, a position may, without change in multiplicity or site symmetry, simulate a position that actually exists, with a different site symmetry, in another point group within the same crystal system. The site set that is realized in the latter position is called the limiting site set. Example. In point group 23, the co-ordinates xyz may be made to obey the conditions $y=x, z=0$; the general position :xyz: then simulates the special position : $x x 0$ : of point groups $m 3 m$ and 432 . In this limiting case the site set in : $x x 0$ : is the limiting site set that is normally realized in the other point groups; it will be designated by the same site-set symbol, which, however, will be enclosed between parentheses. 1

The site set realized in the general position will be called general site set; that realized in any special position, special site set. This terminology is analogous to one that is applied to crysta? forms

1 In Table 9 (point group 23) the parentheses around $12 \mathrm{xx}$ recall the fact that $12 \mathrm{xx}$ occurs as a limiting site set of $6 z 2 x y$. The multiplicity and the site symmetry of position:xyz:, where $6 z 2 x y$ is realized, remain the same in column:xxa:, where (12xx) occurs. in morphological crystallography. ${ }^{1}$

A limiting site set can occur only in a special position in certain point groups, but not ever special position in a given group can receive a limiting site set (see Tables 9-15).

In the Tables the site symmetry is given in its oriented form. In an oriented symbol dots stand for Blickrichtzongen that are not used. In the hexagonal crystal system, for instance site symmetry $m$ may appear as $m . .$, . .m., or ..m, according as the mirror is the primary mirror, one of the secondary, or one of the tertiary mirrors. In the rhombohedral crystal system, the $m$ of the oriented symbol must always appear in the second column of the two-column symbol (.m).

The oriented symbol is easily recognized as the Hermann-Mauguin symbol of the site symmetry, simply by omitting the dots. By taking the dots into account, the elements of symmetry of the site are identified among those of the point group. Examples. In the tetragonal system, $2 m m$. indicates that the 2 is a subgroup of the 4 , the two mirrors are the axial mirrors (represented by a single letter in the second column of the tetragonal symbol) and the dot expresses the fact that the diagonal mirrors of the tetragonal symmetry are not part of this site symmetry. Note that $2 \mathrm{~mm}$. is the tetragonal way of writing the orthornombic mm2; in both symbols the 2 -fold axis is directed vertically, that is, parallel with $z$.

Systems of co-ordinate axes.- The co-ordinate axes used in the present Tables are those of International Tables (1952). In the monoclinic crystal system, we follow the 1951 Stockholm convention ( $b$ unique). In the orthorhombic crystal system, the three columns of a symmetry symbol refer to $a, b, c$, in this order. In the tetragonal crystal system, the smallest cell is used, so that provision must be made

1 Note, however, a different usage in Int. Tab. (1935), where the crystal forms are listed on pp. 49-63: here the term "special form" is used in all limiting cases, while "general form" applies to all other forms. We follow the older conventional usage: general form $\{h k l\}$, a form whose faces are oblique to every element of symmetry; any other form is a special form. 
for the two settings $\overline{4} 2 m$ and $\overline{4}_{m} 2$; the co-ordinate axes being taken along the cell edges. In the hexagonal crystal system, the smallest cell is used and designated $\mathrm{P}$ (formerly called $\mathrm{C}$ ); as in the tetragonal system, provision must be made for two possible settings, $\overline{6} m 2$ and $\overline{6} 2 m$. The rhombohedral crystal system is treated twice: once in rhombohedral co-ordinates, the co-ordinate axes being taken along the lower edges of the primitive cell, so that they look like the three legs of an inverted stool, and with the $x_{r}$ axis pointing towards the observer; and again in hexagonal co-ordinates, with a triple cell resulting from the so-called "R centering". The relations between the two systems of co-ordinates are shown in Intemational Tabies (1952, vol. 1, p. 20, Fig. (a)), where the orientation of the rhombohedral axes with respect to the hexagonal ones is called obverse, positive or direct. The additional nodes are $\mathrm{placed}$ at $2 / 3,1 / 3$, $1 / 3$ and $1 / 3,2 / 3,2 / 3$, so that the face (10T1) slopes toward the observer.

Al1 our systems of co-ordinates are right-handed.

\subsection{Rules of Splitting.}

If the point at the fixed origin is made to move a way from it, the symmetry operations that are acting on it will generate $\mathrm{N}$ equivalent points. This is what we mean when we say that the point at the origin split splits into the $\mathrm{N}$ points. If the point at the origin is made to stay on an element of symmetry as it moves away from the origin, partial splitting takes place, and furtier splitting will be necessary to reach complete splitting, when the site symetry of the final points is reduced to identity.

Splitting of points is the basis of our symbolism of site sets. It will be carried out according to the following rules (see Table 1).

First Splitting.- Two cases are distinguished according as the point group does or does not possess at least one homopolar axis; in the second case the point group possesses a single polar axis (also called heteropolar axis).

A. At least one homopolar axis. In this case there exists only a single point, taken as the origin, that is invariant under all the symetry operations of the point group.

$A(a)$. In point groups $\overline{1}, \overline{4}$ and $\overline{3}$, complete splitting is immediate; the first splitting yields, respectively, the 2,4 and 6 sites of the general position. $n(b)$. In $2 / m, 4 / m, 3 / m(=\overline{6})$ and $6 / m$, as well as in settings $4 m 2,42 m, 3 m 1,31 m$ and $3 m$ (which is treated in rhombohedral co-ordinates), the splitting point is made to stay in a mirror, which is either normal to, or contains, tire principal axis.

$A(c)$. In the remaining point groups (Table 1 , column 1), the point is made to move in the plane that passes througin the origin and is perpendicular to a primary symmetry axis; in addition the moving point must retain the highest possible site symmetry, that is to say, it must move along a secondary or tertiary symmetry axis, or along another primary symetry axis if available (along $x$ or $x \bar{x}$ in noncubic groups, along $z$ in cubic groups).

This moving point reaches a site that is designated by its literal co-ordinates; symmetry related points move symuetrically and complete the assemblage of equivalent sites produced by the first splitting.

B. A single polar axis. When there exists more than one invariant point, the fixed origin of the point group is not completely determined by the symmetry. One invariant point is arbitrarily chosen as origin. The first "splitting" then consists in letting the point move in the invariant space: in point group 1, the moving point can reach any point in three-dimensional space; in point group $m$, any point in the mirror; in any other hemimorphic point group, any point on the polar symmetry axis.

In both cases $A$ and $B$, the first splitting is described by the number of equivalent points, followed by the variable co-ordinates of one of the points. Examples: $2 x y z, 4 x z, 2 x z, 4 x, 6 x ; 1 x y z, 1 x z$, $1 y, 1 z, 1 \times x x$ (Table 1). Note that, in the cubic system, in order to bring out analogies with the tetragonal system, the co-ordinate chosen to symbolize the first splitting is $z$, and the resulting sixpointer ${ }^{1}$ is $6 z$.

Second Splitting.- If, after the first splitting, the site symmetry is not the identity, the site set that was produced by this splitting becomes a generating site set, in that its sites will serve as starting points for further splitting.

1 In crystal morphology the cube, or hexahedron, is the crystal form that corresponds to the six-pointer 6z. The Dana System of Mineralogy symbolizes the form as $\{001\}$ in preference to $\{100\}$, which is the symbol used by many other authors. similar problems call for similar solutions! 
A. If the site symmetry contains a single element $(m, 2,3,4$ or 6$)$, only one more splitting, by means of this element, is needed to bring the site symmetry down to 1 .

B. In every remaining case, the site symmetry consists of $n$ mirrors intersecting in an $n$-fold axis. If the mirrors are of one kind, the second splitting takes place in these mirrors (example: $3 x \vec{x}$ in $3 m 1$, but $3 x$ in $31 \mathrm{~m})$. If the mirrors are of two kinds, the point is made to move in the mirror that contains the next co-ordinate axis, in the cyclic sequence (example: splitting $2 x$ follows splitting $1 z$ in $m m 2$ ). In case $B$, a third splitting is necessary to reduce the site symmetry to 1 .

Third Splitting.- When the generating point still lies in a mirror after the second splitting, the third splitting takes it off the mirror, by making use of the third co-ordinate. Although the last splitting is taking place astride the mirror, the two resulting points need not lie on a line perpendicular to the mirror. This V-shaped splitting simplifies the use of the third co-ordinate. The site symmetry is now reduced to 1 (Table 1).

The final site-set symbol, after complete splitting, is given (column 9, Table 1) for each of the 32 crystallographic point groups and for both settings wherever two settings are possible. The rhombohedral co-ordinates (Miller axes) are provided for, as well as the hexagonal ones (Bravais axes), in trigonal point groups.

\subsection{Special Positions}

In the process of deriving the site-set symbols for the general positions in the crystallographic point groups, a number of special positions have been encountered as intermediate steps. Additional special positions occur in certain point groups.

Some of the additional special positions possess one degree of freedom and, in them, the site symmetry reduces to a hemimorphic subgroup of the point group. The symbols of the corresponding site set are given here, each one followed by the oriented symbols ${ }^{1}$ of its possible site symmetries, enclosed between parentheses: in the cubic point groups, $8 \times x \times(.3 m, .3$.$) ,$ $4 x \times x(.3 m, .3),. 12 x x(m . m 2, . .2)$; in the hexagonal point groups, $2 z(6 m m, 3 . m, 3 m ., 6 \ldots, 3 .),. 6 x \bar{x}$ ( $m m 2$, ..2); in the tetragonal point groups, $2 z(4 \mathrm{~mm}, 2 \mathrm{~mm}$.,

1 See $\mathrm{Sn} .1 .1$ 2.mm, 4.., 2.. ); $4 \times x(m . m 2, . .2)$; in the rhombohedral point groups, $2 x \times x(3 m, 3),. 6 \times \bar{x}(.2)$. Note that the hemimorphic symmetries, $2,4,3,6, \mathrm{~mm} 2,4 \mathrm{~mm}, 3 \mathrm{~m}, 6 \mathrm{~mm}$, are given their oriented symbols in the above 1 ist.

other special positions have two degrees of freedom and, in them, the site symmetry goes down to $m$, either..m in $6 z 4 x x$ (cubic), 1z4xx, $4 x \times 2 z$ (tetragonal), $6 \times 2 z$ and $3 \times 2 z$ (hexagonal); or . $m$. in $4 \times 2 z$ (tetragonal), $1 z 6 x \bar{x}, 6 x \bar{x} 2 z$ and $3 x \bar{x} 2 z$ (hexagonal).

Note that the letters $z, x, y$ that occur in a site-set symbol express successive degrees of freedom, as opposed to co-ordinates of points. Note also, however, that points on the Zwischen axes are symbolized $3 x \bar{x}$, where the letter $x$ indicates that the first degree of freedom is being used and $x \bar{x}$ indicates that the second co-ordinate is negative and has the same numerical value as the first one.

\section{DESCRIPTION OF SITE SETS}

The site sets occur in the 32 crystallographic point groups as assemblages of equivalent points. These symmetry-related points lie on a sphere around the origin. If they are considered to be the poles of crystal faces, the assemblage is a representation, in spherical projection, of a crystal form. The lines radiating from the origin to the face poles would thus be face normals. If, on the other hand, the symmetry-related points on the sphere are considered to be the vertices of a polyhedron, the assemblage becomes a representation of the co-ordination around the origin. In the polyhedron that idealizes a crystal form, the faces are equivalent, and the polyhedron is called isohedraz; in the co-ordination polyhedron the vertices are equivalent, and the polyhedron is called isogonal (referring to the solid angles at the vertices). The two polyhedra are thus dual of each other.

It follows that we have two ways of describing co-ordination of sites around the origin: (1) the co-ordination polyhedron of the chemists; (2) the dual polyhedron, whose face normals are the equivalent elements.

The site sets are shown in stereographic projections (Figs. 1-47); they are classified on the basis of their multiplicity. A site set of multiplicity $N$ is called an N-pointer. It is easy to see how the crystal-form polyhedron gives rise to the co-ordination polyhedron and to coin a name or provide a description for the latter. 
2.1 The One-pointer.- The only one-pointer is the IOINT (Fig. 1), corresponding to the MONOHEDRON (or pedion).

2.2 The Two-pointers.- The two two-pointers are the LINE SEGMENTS, one of wicil is collinear with the origin (Fig. 2) and corresponds to the PINACOID (or parallelehedron), while the other is not collinear with the origin (Fig. 3) and corresponds to the DIHEDRON ${ }^{1}$.

2.3 The Three-pointers.- The two three-pointers are the IRIGONS (or equilateral triangles), one coplanar with the origin (Fig. 4), the other not (Fig. 5). Their duals are, respectively, the TRIGONAL PRISM and tile TRIGONAL PYRAMID.

2.4 The Four-pointers.- There exist seven fourpointers: two RECTANGLES, one coplanar with the origin (Fig. 6) (dual, RHOMBIC PRISM), the other not (Fig.7) (dual, RHOMBIC PYRAMID); two TETRAGONS (or squares), likewise coplanar with 0 (Fig. 8) or not (Fig. 9) (duals: TETRAGONAL PRISM and TETRAGONAL PYRAMID, respectively); and three tetrahedra, which have the same names as their duals: RHOMBIC TETRAHEDRON (or disphenoid) (Fig. 10), TETRAGONAL TETRAHEDRON (or disphenoid) (Fig. 11), and REGULAR TETRAHEDRON (Fig. 12).

2.5 The Six-pointers.- Eight six-pointers comprise: two TRUNCATED TRIGONS, coplanar with 0 (Fig. 13) (dual, DITRIGONAL PRISM) or not (Fig. 14) (dual, DITRIGONAL PYRAMID); two HEXAGONS, coplanar with 0 (Fig. 15) or not (Fig. 16), corresponding to the HEXAGONAL PRISM and HEXAGONAL PYRAMID, respectively; the OCTAHEDRON (Fig. 17), whose dual is the CUBE (or hexahedron); the TRIGONAL ANTIPRISM (Fig. 18), whose dual is the RHOMBOHEDRON (in crystal-form language, a trigonal antiprism is described as the combination of a rhombonedron with the pinacoid); and two more co-ordination polyhedra tinat are vest described as combinations of forms: TRIGONAL PRISM WITH PINACOID (Fig. 19) and TRIGONAL TRAPEZOHEDRON WITH PINACOID (Fig. 20), whose duals are the TRIGONAL DIPYRAMID and TRIGONAL TRAPEZOHEDRON, respectively.

\subsection{The Eigint-pointers.- The seven eight-pointers}

1 The single term dihedron replaces the two terms used by Groth, dome and sphenoid. A crystal form should have the same name regardless of the face symmetry (Boldyrev, 1925). and their duals are as follo:vs: TRUNCATED TETRAGONS, coplanar with 0 (Fig. 21) or not (Fig. 22) (DITETRAGONAL PRISM and DITETRAGONAL PYRAMID); three TETRAGONAL site sets that are described by combinations of TETRAHEDRON (Fig. 23), PRISM (Fig. 24), and TRAPEZOHEDRON (Fig. 25), each with the PINACOID, corresponding to TETRAGONAL SCALENOHEDRON, TETRAGONAL DIPYRAMID and TETRAGONAL TRAPEZOHEDRON, respectively; the RECTANGULAR PARALLELEPIPEDD (Fig. 26)(RHOMBIC DIPYRAMID); the CUBE (OCTAHEDRON) (Fig. 27).

2.7 The Twelve-pointers.- Ten twelve-pointers, six of which belong to the nexagonal system and four to the cubic system, have the following designations. The names of the duals are enclosed in parentheses. TRUNCATED HEXAGON, coplanar with 0 (Fig. 28) (DIHEXAGONAL PRISM) or not coplanar with O (Fig. 29) (DIHEXAGONAL PYRAMID); PINACOID WITH RHOMBOHEDRON (Fig. 30) (HEXAGONAL SCALENOHEDRON); TRUNCATED TRIGONAL PRISM WITH PINACOID (Fig. 31) (DITRIGONAL DIPYRAMID); HEXAGONAL PRISM WITH PINACOID (Fig. 32) (HEXAGONAL DIPYRAMID); HEXAGONAL TRAPEZOHEDRON WITH PINACOID (Fig. 33) (HEXAGONAL TRAPEZOHEDRON); TRUNCATED TETRAHEDRON (Fig. 34) (TRIGON-TRITETRAHEDRON) ${ }^{1}$; CUBE WITH TWO TETRAHEDRA (Fig. 35) (TETRAGON-TRITETRAHEDRON) ${ }^{1}$; CUBOCTAHEDRON (Fig. 36) (RHOMB-DODECAHEDRON); PENTAGONTRITETRAHEDRON WITH TWO TETRAHEDRA (Fig. 37) (PENTAGONTRITETRAHEDRON); DIHEXAHEDRON WITH OCTAHEDRON (Fig. 38) (DIHEXAHEDRON Or PENTAGON-DODECAHEDRON).

2.8 The Sixteen-pointer.- The only sixteen-pointer is a combination of the TRUNCATED TETRAGONAL PRISM WITH THE PINACOID (Fig. 39), whose dual is the DITETRAGONAL DIPYRAMID.

2.9 The 24-pointers.- There exist six 24-pointers. They are listed here with the dual polyhedron shown between parentheses: TRUNCATED HEXAGONAL PRISM WITH PINACOID (Fig. 40) (DIHEXAGONAL DIPYRAMID); TRUNCATED CUBE (Fig. 41) (TRIGON-TRIOCTAHEDRON) ${ }^{2}$; RHOMBICUBOCTAHEDRON (Fig. 42) (TETRAGON-TRIOCTAHEDRON) ${ }^{2}$; CUBE WITH TWO TETRAHEDRA (Fig. 43) (HEXATETRAHEDRON); TRUNCATED OCTAHEDRON (Fig. 44) (TETRAHEXAHEDRON); CUBE WITH OCTAHEDRON AND DIHEXAHEDRON (Fig. 45) (DIDODECAHEDRON); SNUB CUBE (Fig. 46)(PENTAGON-TRIOCTAHEDRON).

1 The two polyhedra belong to the same site set $6 z 2 \times x$ (Table 2).

2 Two two polyhedra belong to the same site set $6 z 4 \times x$ (Table 2). 
2.10 The 48-pointer.- The only 48-pointer is the TRUNCATED CUBOCTAHEDRON (Fig. 47), whose dual is the HEXAOCTAHEDRON.

Note that, in the above nomenclature, a truncated polygon is symmetrically truncated on the angles, a truncated polyhedron (tetrahedron, cube, octahedron) is likewise symmetrically truncated on the corners, but a prism, being an open form with no vertices, is truncated on its edges. The rhombicuboctahedron results from the combination of a cube with octahedron and rnomb-dodecainedron. The snub cube is a cube modified by octahedron and pentagon-trioctahedron. The truncated cuboctahedron shows the twelve rhomb-dodecahedral faces truncating the twelve corners of the cuboctahedron.

The site sets that occur in the 32 crystallographic point groups are listed in Tables 2 to 8 , 1 imiting cases not included. Under a subheading consisting of the names of the co-ordination polyhedron and dual crystal form, the symbol of the site set is followed by the co-ordinates of the equivalent sites, expressed in a condensed manner (see below), and the symbols of the positions where the site set occurs. The position is given by the point-group symbol and the position letter between parentheses. This letter is taken from Carl Hermann's description of the point groups (so-called crystal classes) ${ }^{1}$.

The multiplicity of each site set can be obtained immediately by making the product of the numerals that indicate splitting in the site-set symbol. Example: $6 z 2 x$ has multiplicity 12. No explicit mention of the multiplicity is made in the Tables. A given site set may occur in different positions, in the same point group or in different point groups, and can be described by different symbols, which will be found listed under one another, together with the co-ordinates of the sites and the positions of occurrence. When a site-set symbol corresponds to two names, these are 1 isted under each other in the subheading. Alternate orientations of point groups $(\overline{4} m 2, \overline{4} 2 m$, for instance) have been taken into consideration. The five trigonal point groups are listed twice: once in rhombohedral co-ordinates (Miller axes) under rhombohedral system and again in hexagonal co-ordinates (Bravais axes) under hexagonal system.

The above presentation is largely based on the Report of the Kiel Symposium on Co-ordination Poly- hedral, especially as regards the nomenclature of the polyhedra and the occurrence of the site sets in point groups. For additional data, such as topological and metrical site-set symbols, and limiting cases, this Report should be consulted.

The following remarks will help in the understanding of the tabular presentation.

Condensed description of the site co-ordinates. Instead of listing the co-ordinates of all the equivalent sites in the position, it may be advantageous to use a scheme of condensation. One triplet of coordinates specifies one of the sites of the position, various signs explain how the other sites are derived from the first.

The Greek letter kappa $(x)$ stands for the initial of kyklos, circle, and indicates the three cyclic permutations of the preceding triplet or of two (or more) triplets enclosed between parentheses.

The Greek letter pi $(\pi)$ indicates the six complete permutations of the preceding triplet.

The Greek letter tau $(\tau)$ is used to signify that the first two co-ordinates must be interchanged.

In the hexagonal system when three co-ordinates are used $\left(a_{1}, a_{2}, c\right.$ axes) (in contradistinction to Weber's four co-ordinates), the Greek letter iota (2) indicates the following operations: $(x y z)_{1}=x y z$; $\bar{y}, x-y, z ; y-x, \bar{x}, z$. (They correspond to the cyclic permutations of the first three co-ordinates in a fourco-ordinate symbol in which the third co-ordinate is zero: $x y 0 ; 0 x y=0-y, x-y, y-y ; y 0 x=y-x, 0-x, x-x$.

The \pm sign, which snould be read as "plus and minus", is the expression of centrosymmetry; it doubles the number of equivalent sites. The \pm sign may precede all other statements or a single letter (as in $\pm x, y, z$ ).

Triplets of signs (example: +--) can be followed by a Greek letter, with the same obvious meaning.

Example: the last site set in Table 2 is the 48pointer; the 48 triplets of co-ordinates that would be necessary to give all the co-ordinates explicitly can be condensed into the statement

$$
\pm(+++,+--x)(x y z \pi) \text {, }
$$

which is easily deciphered as follows: take the six complete permutations of the triplet $x y z$, first with the signs +++ and then with the three cyclic permutations of +--; double the number of points, from 24 to 48 , by changing all the signs. Another way of

1 Chapter III in Int. Tab. (1935).

1 J.D.H. Donnay, E. Hellner and A. Niggli (1964). 
condensing would be $\pm(x, y, \pm z ; x, \bar{y}, \pm z) \pi$, which is just as easily interpreted. We consider such statements helpful in evoking the picture of the assemblage of points. Stimulating and interpretative, as well as space-saving, they are preferable to the bland catalog,

$\begin{array}{llllll}x y z & y z x & z x y & y x z & x z y & z y x \\ x \bar{y} \bar{z} & y \bar{z} \bar{x} & z \bar{x} \bar{y} & y \bar{x} \bar{z} & x \bar{z} \bar{y} & z \bar{y} \bar{x} \\ \bar{x} y \bar{z} & \bar{y} z \bar{x} & \bar{z} x \bar{y} & \bar{y} x \bar{z} & \bar{x} z \bar{y} & \bar{z} y \bar{x} \\ \overline{x y} z & \bar{y} \bar{z} x & \bar{z} \overline{x y} & \bar{y} \bar{x} z & \bar{x} \bar{y} y & \bar{z} \bar{y} x \\ \bar{x} \bar{y} \bar{z} & \bar{y} \bar{z} \bar{x} & \bar{z} \overline{x y} & \bar{y} \bar{x} \bar{z} & \bar{x} \bar{y} \bar{y} & \bar{z} \bar{y} \bar{x} \\ \overline{x y z} & \bar{y} z x & \bar{z} x y & \bar{y} x z & \bar{x} z y & \bar{z} y x \\ x \bar{y} z & y \bar{z} x & z \bar{x} y & y \bar{x} z & x \bar{z} y & z \bar{y} x \\ x y \bar{z} & y \bar{x} & z x \bar{y} & y x \bar{z} & x z \bar{y} & z y \bar{x},\end{array}$

which dulls the senses and discourages visualization.

In the description of hexagonal site sets, and of rhombohedral ones in hexagonal co-ordinates, we recommend the elegant Weber four-index symbol $x y j z$ for the co-ordinates of points. All four Bravais axes must be used: the third co-ordinate, $j$, which is made equal to the negative sum of the first two, is not superfluous: it cannot be left out at will, as can the $i$ index in a face symbol ( $h k i z$ ). The advantage of these symbols (Weber, 1922; Donnay, 1947a, b) is that permutations of the first three co-ordinates are made available to express the symmetry of the point assemblage. In fact, the parallelism between the presentation of the co-ordinates $x y j z$ of equivalent points and that of the indices hkiz of equivalent faces is so perfect that the two symbols are interchangeable. This property is the consequence of the mathematical symmetry of the equation of zone control in four-index symbolism,

$$
h x+k y+i j+z_{z}=0,
$$

which expresses the condition for the face (hkil) to be contained in the zone [xyjz].

For calculations, however, symbols with three coordinates are more convenient. For this reason they are used in Table 3 . The transformation from fourindex to three-index symbols is immediate; it consists in subtracting the third co-ordinate $j$ from each of the first three, so that $x y j z$ becomes $x-j, y-j$, $(0)$,$z . Example: 5 T 42$ gives $5-4, T-4,2=932$. Conversely, transforming from three to four co-ordinates: 932 gives $9-(9+3) / 3,3-(9+3) / 3,-(9+3) / 3,2=5 \overline{1} \overline{4} 2$.

It may not be superfluous to stress that any coordinate can be assigned negative or positive values. It follows that the single point of the site set $1 z$, which may have a negative as well as a positive value for $z$ in the co-ordinate triplet $00 z$, corresponds to two crystal forms in morphology: the upper monohedron
$\{001\}$ and the lower monohedron $\{00 \overline{1}\}$. This is the reason why two positions are listed in such a case. Example: 4 rom(a) and 4rm(b) (Table 5).

\section{OCCURRENCES OF SITE SETS}

Site sets occur in positions in point groups. For each crystal system (Tables 9-15), we give a double entry tabulation: each row stands for one point group, each column for a type of co-ordinate triplet. At the intersection of row and column, the symbol of the site set ${ }^{1}$ appears over the corresponding site symmetry of the occupied position. This site symmetry is symbolized in the format of the point-group symbol, that is to say that it is oriented with respect to the symmetry directions of the crystal lattice (see Sn. 1.1).

\section{SUBGROUP RELATIONS}

The relations between groups and subgroups among the 32 crystallographic point groups have been presented, in the form of a line diagram, by Carl Hermann in the 1935 edition of the International Tables.

This diagram is reproduced here as Fig. 48.

\section{SITE SETS AND THEIR OCCURRENCES IN ORIENTED SUBGROUPS}

\subsection{Cubic Degradation (Tab7e 16).-}

The top lines show the site sets and the positions in the cubic point groups. Going down in the first column, we list the various subgroups in order of decreasing symmetry. The tetragonal point groups, for instance, are listed in the first column in their cubic orientation; they are repeated in the second column in their standard tetragonal notation.

Further down the Table, the trigonal point groups are likewise shown twice: as subgroups of the cubic groups, in cubic orientation, in the first column and as standard trigonal point groups, in the third column.

The orthorhombic point groups appear three times: in the first column, as oriented subgroups of the cubic symmetries; in the second column, as oriented

1 or the symbol of the limiting site set, which is written between parentheses where it appears as a limiting case (see Sn. 1.1). 
tetragonal subgroups; and, in the fourth column, as orthorhombic point groups in their own orientation.

The same scheme is carried out all the way down to the triclinic point groups.
5.2 Hexagonal Degradation (Table 17).-

The subgroups of the hexagonal holohedry are listed, including the orthorhombic, monoclinic, and triclinic point groups. 


\section{GENERALITIES}

\subsection{Definitions.-}

A configuration means a set of $n$ specified ${ }^{1}$ congruent primitive lattices, whose nodes are symmetryrelated sites in at least one of the 230 space groups. Every one of the $n$ lattices may be represented by one node that is a site in the conventionally chosen cell. Every site in the configuration is invariant under certain symmetry operations, whicn constitute a subgroup of the point group to which the space group belongs. The subgroup, which may be the trivial subgroup 1, is called point symmetry of the site or site symmetry. A site may happen to lie on an element of symmetry whose operation involves a translation component (a screw axis or a glide plane of symmetry), but such an element cannot be part of the site symmetry, which by definition is a point group.

In a given space group, two configurations are said to occupy one and the same point position (or position for short) if, to every site in the one configuration, there corresponds, in the other configuration, a site that lies on the very same elements of site symmetry.

The condition for two positions, in tile same space group or in different space groups, to belong to the same lattice complex (or complex for short) is that, to every configuration of the first position, there corresponds, in the second position, a congruent or enantiomorphous, configuration -- and conversely. As a consequence of this definition, all the positions in which a lattice complex can be realized occur in one and tine same crystal system. ${ }^{2}$

The positions that belong to the same lattice

1 Specified in the sense that the co-ordinates of the nodes have specified numerical values, in any convenient system of absolute co-ordinates.

2 The two lattice complexes $t P$ and $c P$ are distinct complexes because $t P$ (class of all the sets of points that occupy the nodes of a tetragonal primitive lattice) contains, in addition to all the configurations of $\mathrm{cP}$, configurations that are not included in $\mathrm{cP}$, i.e. configurations of points that do not occupy the nodes of a cubic primitive lattice. complex, eitier in a given space group or in different space groups, may be differently oriented and their site symmetries may also be different. Such positions constitute different representations of the lattice complex.

One of the representations of a lattice complex, in tire space group where it sinows the highest site symmetry, is cnosen as the standard representation. Our choice follows, in general, that already made by Carl Hermann (Int. Tao., 1935). The multiplicity of a lattice complex is the number of its equivalent points comprised within the conventional cell of its standard representation.

General lattice complexes are trivariant; special lattice complexes can be bivariant, univariant or invariant. If a position contains all the configurations of a second position, but the second position does not contain all the configurations of the first, then the lattice complex realized in the second position will be a limiting lattice complex (LLC) of the lattice complex of the first position. The subset of configurations (in the first position) tirat coincide with the set of configurations of the limiting lattice complex will simulate this complex as a limiting case.

Example. In space group 23 the lattice complex that is realized in position $(j)$ :xyz: possesses a subset of configurations that coincides with the set of configurations of another complex, which is realized in positions $(i): x \times 0$ : of space groups $P m 3 m$ and $P 432$. In space group $P 23$, position $(j)$, for $y=x, z=0$, simulates position ( $i$ ) of $P \mathrm{~m} 3 \mathrm{~m}$ and $\mathrm{P432}$, while retaining its own multiplicity and site symmetry.

The lattice complex of only one special position, namely, position $48(f): x 0 \frac{1}{4}$ : in Ia3d is not a limiting lattice complex: it cannot occur as limiting case in any position of any space group. All other special lattice complexes are limiting lattice complexes.

The space group in which a lattice complex is defined in its standard (or normal) representation is called the characteristic space group of the lattice complex. Other representations for which the site symmetry is equal can only occur in the same characteristic space group; other representations, with lower site symmetry, may be found in another space group or in several other space groups, as well as in 
the characteristic space group; nonstandard representations will be referred to as altemate representations.

\subsection{The Lattice-Complex Symbol.-}

6.21 Invariant Lattice Complexes.- An invariant lattice complex, that is to say, a complex that can occupy a parameterless position in a space group, is designated by a short symbol, which consists in a single letter when referring to the standard representation. Example: in the anorthic (triclinic) system (Table 25), $P$ is the symbol of an invariant lattice complex in standard representation, which occurs in space group $\bar{P} \overline{1}$ as position $(\alpha)$, with site symmetry $\overline{7}$ and multiplicity 1. $P \bar{j}$ is its characteristic space group.

The same lattice complex occurs in an alternate representation in $P](a)$, with site symmetry 1 . In this space group the complex occupies a general position :xyz: and is symbolized $P[x y z]$. The coordinates are placed between brackets to indicate that they represent another origin for the complex $P$, which remains invariant in spite of this choice of origin.

In the monoclinic system (Table 26) $P$ and $C$ are invariant lattice complexes in standard orientations. Among the lattice-complex letters, we find all the letters that are used to characterize lattice centerings, since the nodes of any lattice, according to definition, are the equivalent points of a lattice complex.

Some of the symbols listed (Table A) for invariant lattice complexes are topological symbols, which means that they can be used in more than one crystal system. The symbol must be expanded in order to specify the system in which the complex is to be considered. This is achieved simply by using the initial of the name of the system as a prefix to the lattice-complex symbol. Examples: CP, hP, rP, tP, $O P, \mathrm{mP}, \mathrm{aP}$. Note that $t$ stands for tetragonal, $r$ for rhombohedral, a for cnorthic (which is used instead of triclinic in order to avoid conflict between initials). The system need not be specified, of course, unless there is danger of confusion. 


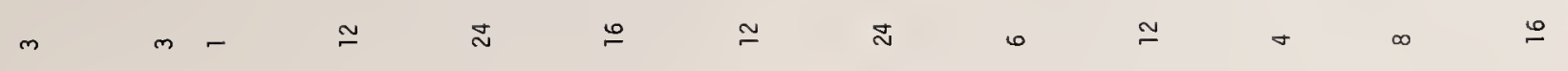
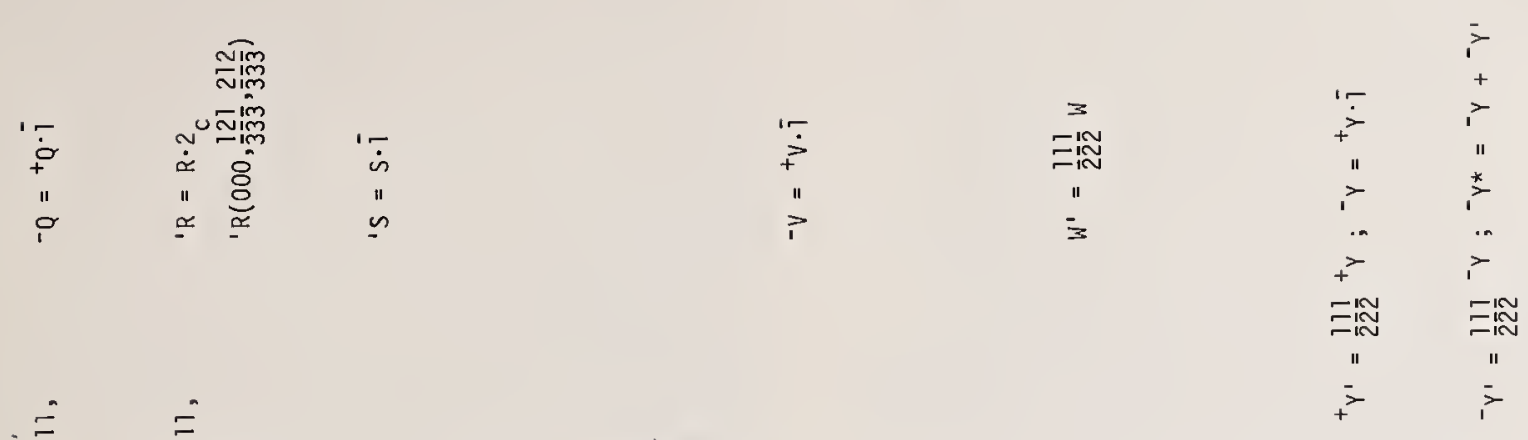

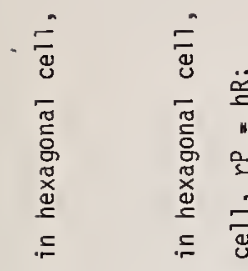

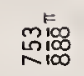

กิ100

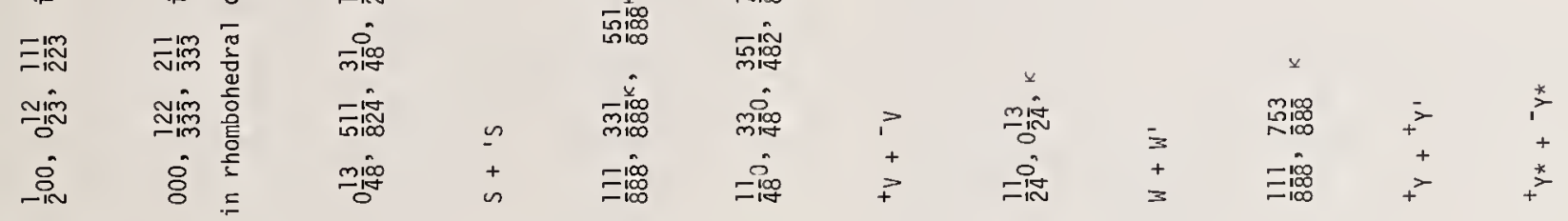

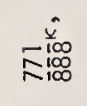

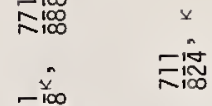

inco

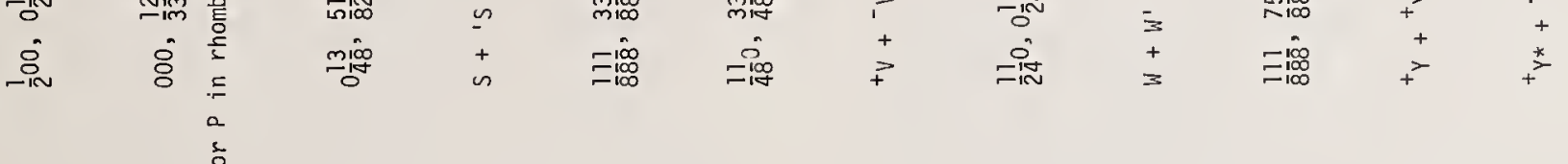

o

$+\infty \quad \propto$
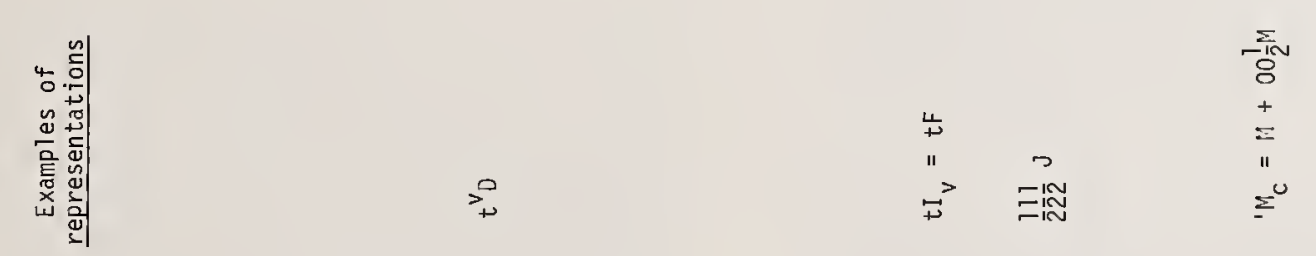

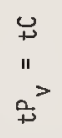

哭

$\underset{5}{5}$

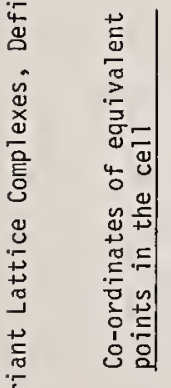

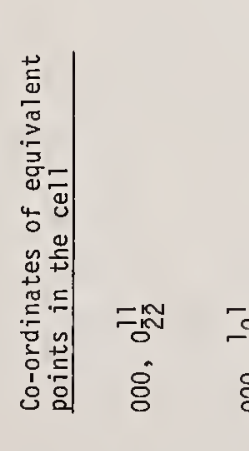

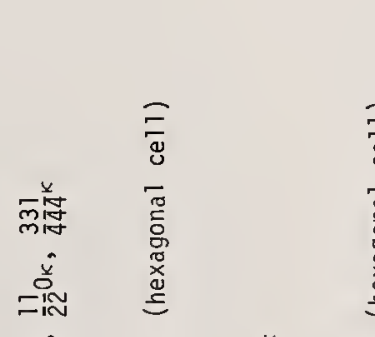

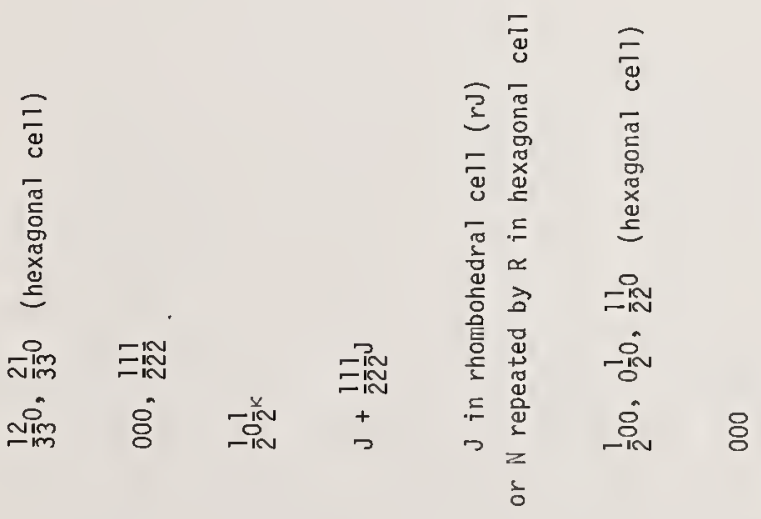

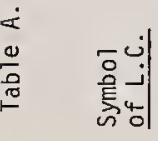

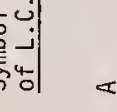

$\infty$ 
Distinction between a lattice complex and its representations.- We may distinguish between the concept of the lattice complex and the various representations that the complex can have in one space group or in different space groups. The standard representation will be designated by the symbol of the lattice complex itself. The symbols of all the invariant lattice complexes in their standard representations are listed alphabetically in Table A (column 1). Examples of alternate representations will be found in column 3. The co-ordinates of the equivalent points in the conventional cell (column 2) and the multiplicity $m$ (column 4) complete the Table.

The symbols used for the invariant lattice complexes are essentially a typographical simplification of tinose that were proposed by Carl Hermann (1960).

The new symbols were agreed upon at the Kiel Symposium (Donnay et al., 1966). They can be remembered without too much difficulty, as each letter chosen recalls some structural feature of the complex. When two representations of a lattice complex of multiplicity $m$ combine to form a new complex with multiplicity $2 m$, the complex letter is usuallyl retained but an asterisk follows it in superscript. Example: J* (pronounce: $\mathrm{J}$ star). If the process is repeated, the complex of multiplicity $4 m$ still has the same letter, this time with a double asterisk. Whenever a complex possesses two enantiomorphous representations, the standard representation is symbolized by a letter preceded by a plus sign in superscript. Example: ${ }^{+} Q$. The representation chosen as standard is the one that contains left-handed screw axes. In this convention, the sign placed in superscript is that of the optical activity.

Alternate representations can be enantiomorphous or congruent to the standard orientation. The minus representation of a plus complex will carry the minus sign in superscript in front of the letter. A congruent representation that is obtained from the standard representation by a translation will retain the same letter, but it will be preceded by the shift vector expressed by its three components in fractions of the cell edges. Examples: $\frac{171}{2} \frac{1}{2} \mathrm{~J}$ is a shifted representation of $\mathrm{J} ; \frac{1}{4} \frac{1}{4} \frac{1}{4} \mathrm{~F}$ and $\frac{3}{4} \frac{3}{4} \frac{3}{4} \mathrm{~F}$ are shifted representations of $F$. A11 other congruent representations will have their symbolic letter of the standard representation preceded by a prime sign. Examples: ' $R$ is

1 Examples of exceptions: $F+\frac{1}{2} \frac{1}{2} \frac{1}{2} F$ is not designated $F^{*}$, it is called $P_{2}$ instead (see below); $\mathrm{P}+\frac{1}{2} \frac{1}{2} \frac{1}{2} \mathrm{P}$ is $I$, not $\mathrm{P} *$. obtained from $\mathrm{R}$ by a $180^{\circ}$ rotation around the $c$ axis (in hexagonal co-ordinates); 's is derived from $S$ by a screw rotation of power 1 or 3 around a $4_{1}$ axis. It is true that ' $S$ can also result from $S$ by an inversion through the origin (or the center of the ce11), but this inversion can always be combined with another operation of the 2 nd kind that is a symmetry operation of the complex to produce an operation of the 1st kind. To obtain a minus representation from the plus representation, on the other hand, the only operation available is one of the 2 nd kind, the inversion through the origin, for instance.

Other alternate representations can be obtained by changing the scale in certain directions (not necessarily all directions), in such a way that the crystal system is preserved. The transformation matrix that expresses the cell edges $\vec{a}^{\prime}, \vec{b}^{\prime}, \vec{c}^{\prime}$ in terms of the edges $\vec{a}, \vec{b}, \vec{c}$, of the complex in standard representation is given in Tables 25-31. Where no matrix is 1 isted, unit matrix is to be understood.

Examples: $P_{b}$ (Table 26) is a representation of $P$ with $b=b^{\prime} / 2$, so that two standard representations of the complex occupy the volume of the cell. The transformation matrix ${ }^{1}$ that gives the cel 1 edges from the edges of the complex is 100/020/001.

A small letter in subscript thus indicates that the corresponding edge of the complex is to be multiplied by 2 in order to give the length of the cell of the lattice in which the complex is represented. Two small letters in subscript, as in $\mathrm{P}_{a b}$, mean that both $a$ and $b$ are to be doubled to give the dimensions of the cell. If all three edges are to be doubled, the subscript used is 2. $P_{2}$, for instance (Table 31 ), is a representation in which the cell volume contains 8 times the $P$ complex in standard representation. The transformation matrix "complex to cel1" is 200/020/002.

Examples of representations that result from a rotation combined with a contraction or an expansion of a mesh are found in the tetragonal system (Table 28) and in the hexagonal system (Table 29-30). Consider a tetragonal $F$ cell, with its 4 nodes. It has twice the volume of an $I$ cell, which is oriented at $45^{\circ}$ to it. The standard representation of the lattice complex is I, with multiplicity 2 . The alternate representation, in the $F$ cell, has multiplicity 4 . The $F$ cell is obtained from the cell of the standard complex by transformation $110 / \overline{1} 10 / 001$. It is rather infelicitous to describe this transformation as a $45^{\circ}$

1 See Sn. 8.1, footnote. 
counterclockwise rotation of the mesh accompanied by an expansion of the lengths in the ratio 1 to $\sqrt{ } 2$. While it is true that, if the $c$ length is kept constant, this peculiar operation will transform the small cell into the large one, it is also true that the same operation will not turn an $I$ cell into an $F$ cell! The symbols proposed by Carl Hermann make use of the letters $v$ and $V$ (from the Latin vertere, to turn) to indicate the $45^{\circ}$ "turn" in the tetragonal system and the $30^{\circ}$ "turn" in the hexagonal system, respectively. We did not feel it would be justified to modify this symbolism, misleading though it might be.

In the above example, the I complex in the $F$ representation could have been designated $I_{v}$. Likewise a $P$ complex in the $C$ representation could have been called $P_{V^{*}}$ (The familiar lattice letters $F$ and $C$ have been retained for the sake of convenience, even though the unity of the symbolism is thereby impaired.)

Consider now a D complex in a tetragonal cell, that is, a tD complex. (In the cubic system, $C D$ is exemplified by the diamond structure.) The tetragonal cell in question will be an $F$ cell, which we will want to describe in the conventional $I$ setting. The transformation matrix $\frac{1}{2} \frac{1}{2} 0 / \frac{1}{2} \frac{1}{2} 0 / 001$ expresses the $45^{\circ}$ clockwise rotation with shrinkage of the cell. The representation of the $D$ complex in the small cell will have multiplicity 4 , instead of 8 , and the complex symbol will be ${ }^{v_{D}}$.

A mnemonic rule may be found useful: to go to a larger cell, the co-ordinate axes $a_{1}$ and $a_{2}$ are rotated counterclockwise, that is, to the right, and the subscript $v$ is placed to the right of the letter; to go to a smaller cell, the co-ordinate axes $a_{1}$ and $a_{2}$ are rotated clockwise, that is, to the left, and the superscript $v$ is placed to the left of the letter. The same rule applies to the hexagonal case, where the capital letter $V$ is used for the $30^{\circ}$ turn.

In the hexagonal system (Table 29-30) one additional symbol is needed whenever the $c$ axis of the $c$ complex in standard orientation is to be multiplied by 3 to give the $c^{\prime}$ length of the cel1. $P_{C}$ indicates this transformation. $P_{C}$ indicates the doubling of $c$. It follows that $P_{C c}$ stands for a multiplication of $c$ by $3 \times 2$ or 6 .

Relations between invariant lattice complexes.Using the abbreviation $P^{\prime}$ to mean $\frac{11}{2} \frac{1}{2} P, F^{\prime \prime}$ to mean $\frac{111}{4} 4 \frac{F}{4} F F^{\prime \prime \prime}$ to mean $\frac{33}{4} \frac{3}{4} F$, and similar abbreviations $F^{\prime}, J^{\prime}, D^{\prime}$, etc., we can write the following equations:

$$
\begin{aligned}
P+P^{\prime} & \left.=I \text { (note tilat } I \text { is preferred to } P^{*}\right), \\
J+J^{\prime} & =J^{*}, \\
F+F^{\prime} & =P_{2}\left(\text { note that } F^{*}\right. \text { is not used), } \\
P+J= & F, \quad P^{\prime}+J^{\prime}=F^{\prime}, \\
F+J^{*}=P_{2}, & F^{\prime}+F^{\prime \prime}=D^{\prime}, \\
h P+G= & h P_{V}=H, \quad h P+N=h P_{a_{1} a_{2} .}
\end{aligned}
$$

Explanation of the symbolic letters used for invariant lattice complexes.- The letters that also describe the variously centered cells of the Bravais lattices are, of course, self-explanatory. The other letters recall some structural feature of the complex, as follows:

D Diamond structure.

E Hexagonal close packing (Italian: esagonale).

The ing atoms in the ing structure occupy the equivalent points of the $E$ lattice complex.

$G \quad$ Graphite structural layer. The $B$ atoms in $\mathrm{AlB}_{2}$ occupy the $G$ complex.

$J$ Defined in standard representation as 3 points in the midpoints of the cell faces. Consider the six half-points within the cell and connect opposite half-points: the resulting figure has the shape of the American toy called "Jack". (The center point of the jack is the center of the cel1, $\frac{1}{2} \frac{1}{2} \frac{1}{2}$.) The atoms of oxygen in perovskite occupy the $J$ complex.

$M$ Complex that results from the repetition of the equipoints of $\mathrm{N}$ by a rhombohedral lattice, in a hexagonal cell. The $\mathrm{Ni}$ atoms in $\mathrm{Ni}_{3} \mathrm{Pt}_{2} \mathrm{~S}_{2}$ occupy the $M$ complex.

$\mathrm{N}$ Net. Three points per mesh, extending into a two-dimensional 4-connected net, are repeated by the hexagonal lattice. The Co atoms in CoSi illustrate the $\mathrm{N}$ complex.

${ }^{+} Q$ Quartz. The Si atoms in high-quartz occupy the ${ }^{+} Q$ complex.

$S$ This complex has site symmetry $\overline{4}$ and contains tetragonal tetrahedra that have 4 symmetry, $\mathrm{S}_{4}$ in Schoenflies notation, formerly called sphenohedral. The Th atoms in $T h_{3} P_{4}$ illustrate the $S$ complex.

$\mathrm{T}$ Tetrahedra sharing all four corners. The $\mathrm{Cu}$ atoms in $\mathrm{MgCu}_{2}$ occupy a $\mathrm{T}$ complex, and so do the oxygen atoms in cristobalite.

${ }^{+} V$ This complex has site symmetry 222 and contains rhombic tetrahedra that have symmetry 222 , or $V$ in Schoenflies notation. The $C a$ atoms in garnet occupy the $V^{*}$ complex $\left(V^{*}={ }^{+} V+{ }^{-} V\right)$. 
Non-intersecting (German: windschief), mutually perpendicular rows of equivalent points. The $\mathrm{Cr}$ atoms in $\mathrm{Cr}_{3} \mathrm{Si}$ occupy the $\mathrm{W}$ complex.

${ }^{+} Y$ In the invariant complexes ${ }^{+} Y+{ }^{+}{ }{ }^{\prime}={ }^{+} Y^{*}$ and ${ }^{-} Y+{ }^{-} Y^{\prime}={ }^{-} Y^{*}$, three equipoints of $Y$ surround one equipoint of $Y^{\prime}$, and vice versa, so as to form the letter "Y". The $\mathrm{Li}$ atoms in $\mathrm{LiFe}_{5} \mathrm{O}_{8}$ occupy the ${ }^{+} Y$ ' complex. The Au atoms in $\mathrm{Ag}_{3} \mathrm{AuTe}_{2}$ occupy ${ }^{+}{ }^{*}$. The $K$ atoms in high-temperature leucite, $\left.K(A] S i_{2}{ }_{6}\right)$, occupy $Y * *$.

As further examples, $J^{*}$ is illustrated by the Pt atoms in $\mathrm{Pt}_{3} \mathrm{O}_{4}, \mathrm{~S}^{*}$ is occupied by $\mathrm{Si}$ in garnet $\mathrm{Ca}_{3} \mathrm{Al}_{2}\left(\mathrm{SiO}_{4}\right)_{3}$, and $\mathrm{W}^{*}$ accommodates the $\mathrm{B}$ atoms in $\mathrm{Zn}_{4} \mathrm{O}\left(\mathrm{BO}_{2}\right)_{6}$.

6.22 Variant Lattice Complexes.- We have seen in Part I that the splitting of the point at the fixed origin of a point group gives rise to a site set, which is an assemblage of equivalent points around the origin. Likewise the points of an invariant lattice complex may be made to split into as many assemblages of equivalent points, the sum of which constitute a variant complex. Examples: Starting from the invariant lattice complex $P$, in the cubic system (Table 31), splitting along the principal symmetry directions of the site symmetry gives $6 z$ (see Table 1, last line) and the result is the univariant lattice complex P6z. If we take I, instead of $P$, as the generating complex, the resulting univariant complex will be $16 z$, which is composed of two octahedra of equivalent points, one around each of the two points of I.

The direction in which splitting is to take place is expressed by using, once, twice, or tnree times, tne letter that designates the variable, as was done for site sets in point groups. Examples: P12xx is a univariant complex composed of points that lie on the bisectrices of the interaxial angles and thus are the apices of a cuboctahedron. I8xxx is also univariant, but the length $x$ is plotted three times in succession, parallel to the three unit vectors $\vec{a}_{1}, \vec{a}_{2}$ and $\vec{a}_{3}$, so that the resultant lies along the body diagonal of the cell and, within the cell, the complex consists of two cubes, one around each of the two points of I, the generating complex. In the orthorhombic system (Table 27), $F 2 y 2 z$ is made up of four site sets $2 y 2 z$, one around each of the four points of the generating complex $F$; it is a bivariant complex, because $y$ and $z$ have different independent numerical values. In the same system, a simple trivariant complex is $P 2 x 2 y 2 z$, the symbol of which is self-explanatory.
It may happen that any one of several lattice complexes could be used as generating complex to produce, by splitting, a given variant complex. Our first task is to set up rules, according to which a unique choice of generating complex can be made in all cases.

\subsection{Rules for selecting the generating lattice com-} plex (GLC).-

The problem may be stated as follows: Given a variant lattice complex, select a generating lattice complex, that is, either an invariant lattice complex (ILC) or a limiting lattice complex (LLC), whose points will by splitting produce the given variant complex. (We note that, if the characteristic space group of the variant lattice complex is different from that of the generating lattice complex, the generating lattice complex can be an ILC or a LLC.)

If more than one generating complex is available, consider the site symmetries of the several possible generating complexes, taken as positions in the space group, and use as many of the following rules as needed.

Rule 1: If the site symmetries are of different orders, take the highest order ( $n$ in Table $B$ ).

\section{TABLE B}

Possible site symmetries for an invariant lattice complex listed according to decreasing Laue symmetry for each order $n$ of the point group and each number $F$ of degrees of freedom

\begin{tabular}{|c|c|c|c|c|}
\hline$n$ & $F=0$ & $F=1$ & $F=2$ & $F=3$ \\
\hline 48 & $4 / m \overline{3} 2 / m$ & --- & --- & --- \\
\hline 24 & $432, \overline{4} 3 m ; 2 / m \overline{3} ; 6 / m 2 / m 2 / m$ & --- & --- & --- \\
\hline 16 & $4 / m 2 / m 2 / m$ & --- & --- & --- \\
\hline 12 & $23 ; 622, \overline{6} 2 m ; 6 / m ; \overline{3} 2 / m$ & $6 m m$ & --- & -- \\
\hline 8 & $422, \overline{4} 2 m ; 4 / m ; 2 / m 2 / m 2 / m$ & $4 m m$ & -- & -- \\
\hline 6 & $\overline{6} ; 32 ; \overline{3}$ & $6 ; 3 m$ & --- & --- \\
\hline 4 & $4 ; 222 ; 2 / m$ & $4 ; m m 2$ & --- & --- \\
\hline 3 & --- & 3 & --- & --- \\
\hline 2 & T & 2 & $m$ & --- \\
\hline 1 & --- & --- & --- & 1 \\
\hline
\end{tabular}

Example: In space group $\mathrm{Pm} 3 \mathrm{~m}$, position $6(e) \times 00$ is the characteristic position for a univariant lattice complex. This LC may be described from two different generating complexes, both of which are invariant lattice complexes: $P$ in $1(a)$ with site symmetry $m 3 m$ and $\frac{11}{2} \frac{1}{2} \mathrm{~J}$ in $3(d)$ with site symmetry $4 / m m m$. The 
order of the group of site symmetry is 48 in $I(a)$ and 16 in $3(d)$. P must be chosen as generating complex, and the univariant lattice complex will be written P6z.

If the site symmetries have the same order, it may be usefut to consider synonyms, that is to say, to provide, for the variant LC, alternate descriptions based on different generating complexes that belong to the same crystal system (see Tables 25-31). Two cases can occur: the site symmetries are different point groups or they are the same point group. The following rules indicate which of the synonyms must be selected in order to get as short a symbol as possible.

Rule 2: If the site symmetries are different point groups of the same order, take the smallest number of degrees of freedom.

Example: To describe the trivariant LC that is realized in the general position $8(e)$ in $\mathrm{Pbcm}$, we choose site symmetry $\bar{T}$ in $4(a), 000$, with no degree of freedom, in preference to $2 .$. in $4(c), x, 1 / 4,0$, which has one degree of freedom, and ..m in $4(d)$, $x, y, 1 / 4$, which has two.

RuTe 3: If the site symmetries are different point groups of the same order and rule 2 does not apply, take the highest Laue symmetry (see Table $B$ and note that $6 / \mathrm{m}$ arbitrarily takes precedence over $\overline{3} 2 / \mathrm{m}$ ).

Example: For $16(n)$ in $P 4 / m e c$, site symmetry 422 in $2(a)$ is chosen in preference to $4 / m$ in $2(b)$ because it has a higher Laue symmetry.

Rule 4: If the site symmetries are different point groups of the same order and rule 3 does not apply, take the group of proper rotations.

Example: For $16(n)$ in $P 4 / n b m$, site symmetry 422 in $2(a)$ takes precedence over $42 \mathrm{~m}$ in $2(c)$.

Rule 5: If the site symmetries are one and the same point group in different orientations, take the coordinate axes of the LC parallel to those of the space group, rather than parallel to the diagonal directions.

Example: For $8(p)$ in $P_{4} 22$, site symmetry 222 oriented 222. in $2(a)$ and $2(c)$ is preferred to 222 oriented 2.22 in $2(e)$; the Neben axes are taken rather than the zwischen axes.

Rule 6: If the site symmetries are one and the same point group in different orientations and rule 5 does not apply, the $x$ direction takes precedence over the $y$ direction, which takes precedence over the $z$ direction.

Example: For $8(d)$ in $\left.I 2{ }_{1}{ }^{2}{ }^{2}\right\}$, site symmetry 2 is available in three orientations; $2 .$. in $4(a)$ is chosen rather than .2 . in $4(b)$ or ..2 in $4(c)$.

Rule 7: If the site symmetries are one and the same point group in only one orientation, give two or more GLC symbols, writing first the GLC obtained by setting $x=y=z=0$ in the co-ordinates of the given variant position. (If there are more than two generating complexes, list all but the first one in alphabetic order.)

Example: In the example given for rule 5, there were two positions with site symmetry 222 ., namely 2(a) with the ILC $P_{c}$ and $2(c)$ with $0 \frac{1}{2} 0 \mathrm{I}$. The symbol ..2 $P_{c} I 2 \times 2 y z$ of the complex of the general position $8(p)$ in $P 42_{2} 22$, therefore, makes use of two generating complexes, of which $\mathrm{P}_{\mathrm{c}}$ comes first.

In Tables 18-24, which relate the lattice complexes to the Weissenberg complexes, symbols are given for alt synonyms. Synonyms that violate rules 2-6 are written in full and placed between brackets. If the same site set can be used after any of the generating complexes, the synonym is given in abbreviated notation. Example: $S *\left[V^{*}\right] 2 x$ instead of $S * 2 x\left[V^{*} 2 x\right]$.

Remark.- The shift vector that is placed in front of the LC symbol to designate a shifted representation of the LC will always refer to the first GLC when there is more than one in the symbol.

\subsection{Distribution symmetry.-}

We have designated $(S n .1 .1)$ the fixed point of a point group by the term high symotry point (HSP). The generation of a variant lattice complex may be visualized as consisting of the following steps: the splitting, into a set of equivalent points, of the HSP of the point group that belongs to the space group under consideration; (2) the subdivision of this set into a number of subsets to be distributed in the cell so that each one becomes associated with one (or more than one) point of the GLC. The subsets may have more than one orientation.

The symmetry operation that carries a subset from near the HSP to its assigned location near a point of the GLC is represented by the symbot of a symmetry element of the space group. Such a symbol will stand for the first power of the symmetry operation if the symmetry element is of order 2; if the order is greater than 2, the power to be used witl be explicitiy stated 
(if no power is stated, all the powers are meant to be used). The symbol of the symmetry element will be oriented by means of dots that stand for the several columns of the Hermann-ilauguin symbol. It will be written in front of the symbol of the GLC. Enough symmetry operations must be stated to specify the orientations of all the subsets. Together they constitute the distribution symmetry, which is not a group.

The following rules have been applied in expressing the distribution symmetries.

Rule 1: All the symmetry elements used to express the distribution symetry should pass between the equivalent points of the GLC.

RuTe 2: The number of symmetry elements used must be kept to a minimum.

Rule 3: The number of transiation components must be kept to a minimum.

RuTe 4: Symmetry planes are to be preferred to axes; axes to center; among axes, $\overline{4}$ to 4 .

Rule 5: In order of preference the directions are $x, y, z$.

Rule 6: Whenever possible, splitting is done from the standard representation of the GLC, except when the chirality of an enantiomorphous GLC is lost in the resulting variant lattice complex.

Example. In $I 4,32$ the sites of $24(f) 2 \ldots$ can be obtained oy splitting from the standard representation ${ }^{+} V$ or from the alternate representation ${ }^{-} V$. (Either one will do.) In such a case, instead of using both representations and writing .3. ${ }^{+} V^{\top} V 2 z$, we omit the sign and write $.3 . v 2 z$. (In so doing, we follow a rule proposed by Carl Hermann.) In the same space group, however, we write $4_{1} .{ }^{-} \gamma * 3 x \bar{x}$ and $4_{3} .{ }^{+} \gamma * 3 x \bar{x}$ for the two univariant lattice complexes realized in $24(g)$ and $24(h)$, each of which retains the chirality of its GLC: ${ }^{-} \gamma *$ and ${ }^{+} \gamma *$, respectively. ${ }^{1}$

Rule 7: In a $C$ centered space group, it is not allowed to bring 000 to coincidence witn $00 \frac{1}{2}$ by an

1 It would be superfluous to repeat the sign in front of the univariant complex symbol and write: $-\left(4_{1} \ldots-{ }_{Y * 3 \times \vec{X}}\right)$ and ${ }^{+}\left(4_{3} \ldots+Y * 3 \times \bar{x}\right)$. operation of the distribution symmetry; the latter must bring 000 onto $\frac{1}{2} \frac{1}{2} \frac{1}{2}$ instead. Likewise in an I centered space group, the distribution symmetry should bring 000 onto $\frac{1}{2} \frac{1}{2} 0$, not onto $00 \frac{1}{2}$.

Rule 7 is necessary to avoid ambiguity whenever $C_{c}$ is the GLC. Without this rule, two different complexes would get the same symbol, namely: ..mc $c^{2 x 2 y z}$ for both $\operatorname{Cecm}(m)$ and Ibam $(k)$. This situation arises from the fact that $C_{c}$ may be constructed as $c+00 \frac{1}{2} C$ or as $I+00 \frac{1}{2} I$.

The complete lattice-complex symbol may contain up to four parts: (1) the generating-lattice-complex symbol, which consists of a letter for an invariant lattice complex (possibly modified by a subscript or a superscript), followed by (2) the site set that indicates the splitting at each point of the GLC, and preceded by (3) the distribution symmetry. To avoid ambiguity it may be necessary to specify the crystal system; this is done by prefixing (4) the initial letter of the name of the system, in lower case, and hyphenating it to the symbol. In addition, in case of a representation symbol, parts (3), (1), (2) may be enclosed in a parenthesis modified by a subscript or a superscript, and the symbol so-far described may be preceded by a shift vector.

\section{WEISSENBERG COMPLEXES AS GENERATING COMPLEXES}

\subsection{The Weissenberg complexes..-}

There exist lattice complexes, 67 in a 11 , for which the multiplicity does not decrease for any special values of the co-ordinates. Such a lattice complex can simulate an invariant lattice complex as $\underline{a}$ limiting complex. These 67 complexes were first recognized by Weissenberg, who gave them the name Houptgitter. We propose to call them the Weissenberg complexes.

The Weissenberg complexes may be regarded as a basic set of generating complexes. By splitting, they lead to 402 lattice complexes. The Weissenberg complexes include all the invariant lattice complexes and also some univariant, bivariant and trivariant ones. All of them, except the trivariant ones, could be used as generating complexes; according to our rules, however, a few of them are not needed. The reason for this is that we give synonyms of lattice-complex symbols only when the site symmetry is of the same muitiplicity for all the possible generating lattice complexes; if we were to give ail possible synonyms, all the Weissenberg compiexes would be necessary. 
7.2 Tables of Weissenberg complexes.-

The Tables of Weissenberg complexes (Tables 1824) give the following information, under each crystal system.

Each Weissenberg complex appears as a subheading, in which its symbol is followed by its characteristic space group, data pertaining to the position it occupies in its standard representation (multiplicity, Wyckoff letter and site symmetry) and the co-ordinates of the equivalent points.

Under this subheading comes the list of all the lattice complexes that are derived by splitting. Each one is given in its standard representation only, its symbol being followed by that of the characteristic space group with Wyckoff position letter, and the coordinates of the equivalent sites.

It may happen that the co-ordinates of the sites of the position given in the second column are not those listed in the third column but are shifted by a constant vector. 1 In such a case, the Wyckoff letter is marked by a prime ('). Example: the sixth lattice complex listed under $P$ in Table 18 is 4.. $P_{2} 3 \times \bar{x}, I 432\left(i^{\prime}\right)$; the co-ordinates given in the International Tables (1952) under I432 (i) are those of the shifted representation $11114 P_{2} 3 \times \bar{x}$.

The co-ordinates are presented in condensed form. Moreover, the several origins that are provided by a centered lattice mode will be indicated by means of the conventional lattice letter: $I+(x x x ; \ldots)$ instead of $\left(000 ; \frac{1}{2} \frac{1}{2} \frac{1}{2}\right)+(x x x ; \ldots)$.

\subsection{Figures of Weissenberg complexes.-}

7.31 Cubic system.- The $\boldsymbol{z}$ co-ordinates are expressed in eighths of the cell edge.

The 16 invariant lattice complexes are figured in standard orientation (Fig. 49). They are repeated, with examples of their representations, on Figs.50-51. The equations that relate the complexes and their representations can be read off these figures.

There are five univariant cubic lattice complexes that are Weissenberg complexes (Figs. 52-53). They are drawn in standard representation, each in its characteristic space group. The full range of values of the parameter that is permitted by the one degree of freedom cannot be shown graphically, but arrows indicate the range between the two or three limiting cases that are used as generating complexes. Example:

1 The shift vector is not given in Tables 18-24; it is specified in Tables $25-31$ and $32-38$.
${ }^{2}{ }_{1}{ }_{1}$. FYlXxx in $22_{1} 3$. In this example, position $(\alpha)$ of $\mathrm{P}^{2} 1^{3}$ has co-ordinates $x x x ;\left[\frac{1}{2} 0 \frac{1}{2}+(\vec{x} x)\right] \pi$. For special values of the co-ordinates, the position simulates two invariant complexes, $F$ and $Y$, each one in four different representations, as follows: for $x=0, \mathrm{~F}$; for $x=1 / 8,{ }^{+} \mathrm{Y}$; for $x=1 / 4, \frac{11}{4} \frac{1}{4} \mathrm{~F}$; for $x=3 / 8, \frac{1}{2} \frac{1}{2} \frac{1}{2}-Y$; for $x=1 / 2, \frac{1}{2} \frac{1}{2} \frac{1}{2} \mathrm{~F}$; for $x=5 / 8$, $\frac{1}{2} \frac{1}{2} \frac{1}{2}+Y$; for $x=3 / 4, \frac{3}{4} \frac{3}{4} \frac{3}{4} F$; for $x=7 / 8,-Y$. According to our rules ( $S n .6 .3$ ), we use $F$ as the first generating complex and $Y$, which stands for the four representations of ${ }^{+} Y$, as the second one. The distribution symmetry ${ }^{2}{ }_{1}{ }_{1} \ldots$ is found by applying the rules (Sn. 6.4). Note that the figure illustrates only the standard representations of $F$ and ${ }^{+} Y$; this is done to avoid cluttering up the drawing.

\subsection{Hexagonal and rhombohedral systems, in} hexagonal description.- The z co-ordinates are expressed in twelfths of the cell edge.

There are 7 invariant complexes: $P, G, E, N,{ }^{+} Q$ in the hexagonal system; $R$ and $M$ in the rhombohedral system (Fig. 54).

Examples of non-shifted representations are i1lustrated (Fig. 55).

There are 5 univariant and 2 bivariant complexes, in these two systems, that are Weissenberg complexes (Figs. 56-57).

In case of a bivariant complex, the range of variation is indicated by hachured triangles. Note that these triangles do not give the complete 2-dimensional range. The height of each triangle can be read at its three corners. Any site inside one of the hachured triangles will have corresponding sites in all the others.

7.33 Tetragonal system.- The $z$ co-ordinates are expressed in eighths of the $c$ cell edge.

The tetragonal invariant lattice complexes are: $P$ (alternate representation $C=P_{v}$ ), I (alternate representation $F=I_{V}$ ), $D$ (which occurs only as $v_{D}$ ), $T$ (which occurs only as $v_{T}$ ). Both $v_{D}$ and $v_{T}$ are used as generating complexes. Another representation of $P$ is $C_{c}$. These tetragonal lattice complexes and the alternate representation $F$ can be found among the figures of the cubic complexes (Figs. 49-51): only the $c / a$ ratio must be taken $\neq 1$. Additional representations $C, C_{C}, V_{D}$, and ${ }^{V_{T}}$ do not appear on the cubic drawings and are presented in Fig. 58 ,

There are eight variant tetragonal Weissenberg complexes: 7 univariant and one bivariant (Figs. 59- 
60). The symbol is that of the complex in its standard orientation; the drawing figures the complex in the representation realized in the position of the space group, and the necessary shifts are indicated in the legend of the signs used to show the equivalent points of the generating complexes. The shift of the first generating complex is thus specified and the symbol of the figured representation can be obtained by prefixing this shift to the given Weissenberg-complex symbol.

\subsection{Orthorhombic system.- The $z$ co-ordinates} are expressed in eighths of the cell edge.

The orthorhombic invariant lattice complexes are: $P, I, F, D, T$, which result from easily visualized deformations of the cubic lattice complexes that are designated by the same letters (Fig. 49), and C, which results from the orthorhombic deformation of the tetragonal C (Fig. 58), itself a representation of the tetragonal lattice complex $P$.

Alternate representations of orthorhombic invariant lattice complexes are as follows: $P_{2}$, the orthorhombic analog of the cubic $P_{2}$ (Fig. 50); $C_{C}$, the orthorhombic analog of the tetragonal $C_{c}$ (Fig.58); and additional representations $P_{a}, P_{b}, P_{c}, P_{b c}, P_{a c}$, $P_{a b}, I_{a}, I_{b}, I_{c}, A, B, A_{b}, A_{a}, B_{b}(F i g .61)$.

There are 5 univariant orthorhombic Weissenberg complexes, 2 bivariant an'd one trivariant (Figs. 6263). Two of them need special explanations.

The bivariant complex $T .2, B_{b} A_{a} F I$ is illustrated in shifted representation by means of two orthogonal projections; the ca projection showing the partial range of site location by means of hachured 2-dimensional fields.

The drawing of the trivariant complex, which is realized in $\mathrm{P}_{7}{ }_{1}{ }_{1}{ }_{1}(a)$, shows partial 3 -dimensional range of site location by means of irregular tetrahedra.

7.35 Monoclinic system.- The $y$ co-ordinates are expressed in eighths of the $b$ cell edge.

There are two monoclinic invariant complexes, $P$ and $C$ in standard representations (Fig. 64). Examples of alternate representations, $P_{b}, P_{c}, P_{a b}$ and $A, I$, $C_{C}, F$; for $P$ and $C$, respectively, are also $i 17$ ustrated tecause they are used in the text (Tables 23, 30 and 37).

There are three variant monoclinic Weissenberg complexes: two univariant and one bivariant (Fig.65).
7.36 Anorthic system.- The anorthic system has only one Weissenberg complex. It is the anorthic $P$, which results from anorthic deformation of any other P.

\section{LIST OF THE LATTICE COMPLEXES AND THEIR OCCURRENCES}

\subsection{Arrangement of the Tables.-}

The crystal systems are listed in order of decreasing symmetry (Tables 25-31).

The tables can have from 2 to 5 columns. The first column lists the lattice complexes, each one followed by its alternate representations. If needed a footnote or a column gives the transformation matrix ${ }^{1}$ from the cell of the standard representation to the cell of the space group in which the occurrences of the alternate representation are being considered. Unit transformations are not explicitiy given.

The next column gives the multiplicity of the lattice complex, in each of its representations, referred to the cell of the space group. Example: in the tetragonal system (Table 28), the multiplicity is 1 for $P, 2$ for $P_{C}$ and $C, 4$ for $P_{a b}$ and $C_{c}$.

The next column gives the site symmetry, oriented in the symbolism of the relevant crystal system. Example: in the orthorhombic system (Table 29), the site symmetry of $\mathrm{P}_{\mathrm{ab}}$ is 222 in Cmma(a) and Cmma(b), $2 / m$. . in Cmma $(c)$ and Cmma(d), .2/m. in Cmma(e) and Cmma(f), ..2/m in Chmom(e) and Crmm $(f)$, and $T$ in $\operatorname{Pban}(e), \operatorname{Pban}(f), \operatorname{Prmm}(c)$ and $\operatorname{Prmm}(d)$.

The last column lists a11 the occurrences of every lattice complex, in its standard and al ternate representations. The first space group mentioned is the characteristic space group and the first Wyckoff letter is that of the position which defines the standard representation. A11 representations that are shifted with respect to the standard one have the shift specified after the Wyckoff letter by its three fractional components.

1 The matrices are presented in the linear form, long ago proposed by T.V. Barker, in which the three rows are written on one line, separated by oblique fraction bars. Example: letting $\vec{a}^{\prime}, \vec{b}^{\prime}, \vec{c}^{\prime}$ be the vectors that define the conventional cell of the space group and $\vec{a}, \vec{b}, \vec{c}$ those that define the cell of the lattice complex in standard representation, the transformation

$$
\vec{a}^{\prime}=\vec{a}, \vec{b}^{\prime}=\vec{b}, \vec{c}^{\prime}=2 \vec{c}-\vec{a}
$$

will. be represented by the matrix 100/010/102 . 
Each crystal system has its table subdivided into four parts, which gather, respectively, the invariant, univariant, bivariant and trivariant complexes. The only exception is the anorthic system, in which only one invariant and one trivariant complex occur.

\subsection{Remark. -}

In the tetragonal system Carl Hermann defined $V_{D}$ and $v_{T}$ in the smaller cell, whereas we define $D$ and $T$ in the larger cell. This difference in outlook accounts for the fact that, in Hermann's tables, the transformation matrix for these two complexes is the unit matrix, whereas it is $\frac{1}{2} \frac{1}{2} 0 / \frac{1}{2} \frac{1}{2} 0 / 001$ in our own tabulation.

\section{LIST OF SPACE GROUPS}

\section{WITH LATTICE-COMPLEX REPRESENTATIONS}

The crystal systems are here listed in order of decreasing symmetry (Tables 32-38).

Each system is subdivided into as many parts as there are point groups in it. Under each point group, the space groups are taken up in the sequence of increasing superscripts in the Schoenflies symbols.

For every space group, we give, for each position, the lattice complex in the appropriate representation. The position data appear under the lattice-complex symbol. In order to save space, when two consecutive positions representing the same lattice complex have the same multiplicity and the same oriented site symmetry, the multiplicity is written in front of the first Wyckoff letter and the site symmetry, behind the second. This typographical presentation retains the rule, followed by the International Tables, that the multiplicity precedes the Wyckoff letter and the site symmetry follows it. The site symmetry is oriented by means of dots, which stand for the unused symmetry directions of the lattice in the crystal system being considered. This scheme is particularly useful to symbolize point groups that belong to lower crystal systems: it helps bring out subgroup relations.

For the sake of brevity, only the standard lattice-complex symbol is given - no synonym, no abbreviated notation of synonyms. The shift vector, of course, cannot be dispensed with. The positions are listed in the alphabetical order of the Wyckoff letters, with a few exceptions. In $\mathrm{Em} 3$, for instance, it is clear that position (h) must immediately follow position (e), since the two positions accommodate the same lattice complex $\mathrm{P} 6 \mathrm{z},(e)$ in its standard representation and $(h)$ in a shifted representation.
The position that serves to define a lattice complex in its characteristic space group is enclosed in a frame. It is the first occurrence of the lattice complex in the characteristic space group. Our choice is, in most cases, the same as that made by Carl Hermann. Note that, when the LC occurs in several representations, the standard one is chosen. Note also that tinere are cases where the LC is defined by means of a shifted representation. Example: in Pcem, position $8(r) 1$. This is a consequence of the choice of origin. In the example given, if the origin were to be chosen at 222 instead of $2 / m$, the standard representation would be obtained.

In any centrosymmetric space group, the origin is chosen at a center, unless otherwise stated.

\subsection{Remarks.-}

Even though our method of expressing the orientation of tine site symmetry enables us to distinguish between $2 .$. and. .2 in the cubic system, some ambiguity persists in each case. Examples: In $\mathrm{P4}_{2} 32$, the oriented site-symmetry symbol is $2 .$. for both I6z, in $12(h)$, and .3.W2x, in 12(i). In I432, bot'h I1 $2 x \mathrm{x}$, in $24(h)$, and $\frac{11}{4} \frac{1}{4} 4 \ldots P_{2} 3 x \bar{x}$, in $24(i)$, have .2 as site symmetry. The differences between the 2-fold axes in the two positions in each space group stem from their relations to the origin.

In the hexagonal system, space group $P 312$, the co-ordinates of the sites of position $3(a)$ were given as follows, in the 1935 edition of the International Tables:

$$
x, \bar{x}, 0 ; \quad x, 2 x, 1 / 3 ; \quad 2 \bar{x}, \bar{x}, 2 / 3 .
$$

In the 1952 edition, the $z$ co-ordinates were increased by $1 / 3$, so that the sites are now written:

$$
x, \bar{x}, 1 / 3 ; \quad x, 2 x, 2 / 3 ; 2 \bar{x}, \bar{x}, 0 \text {; }
$$

and agree with the co-ordinates given for position $3(a)$ in space group $P_{3}{ }^{21}$ in the 1935 edition:

$$
x, 0,1 / 3 ; 0, x, 2 / 3 ; \quad \bar{x}, \bar{x}, 0 \text {. }
$$

In the tetragonal system, space group $P 4 /$ mee, note that P2z occurs in $4(g)$ as $(P 2 z)_{C}$. This should not be written $\mathrm{P}_{\mathrm{C}} 2 \mathrm{z}$ : the subscript must modify the whole $L C$, which is $P 2 z$, defined by position $2(g)$ in its characteristic space group $P 4 / \mathrm{mmm}$.

In $P 4 / m n c$, position $8(g) . .2$, the LC is written $0 \frac{1}{2} \frac{1}{4}$ (.b.c $\left.2 x x\right)_{c}$. Note that the distribution symmetry comes from the characteristic space group $P 4 / \mathrm{mbm}$. The subscript $c$, being attached to the parenthesis, recalls the fact that the LC inside the parenthesis was defined in another space group; in other words, that the space group in which it occurs here is not its 
cnaracteristic space group.

When several lattice complexes can be chosen as generating lattice complexes, we do not explicitly symbolize those that would be designated by the same letter. Example: In $84 /$ nec, $4(c) \overline{4} \ldots$ is described as $0 \frac{1}{2} \mathrm{O}(. .2 \mathrm{CI} 1 \mathrm{z})_{c}$. (The complex has been defined in P4/nmm, position $2(c) 4 m m$, as $0 \frac{1}{2} 0.2 \mathrm{CI} 7 \mathrm{z}$.) If we set $z=0,1 / 8$ and $1 / 4$, successively, in the co-ordinates: $0 \frac{1}{2} z ; \frac{1}{2} 0 \bar{z} ; 0, \frac{1}{2}, \frac{1}{2}+z ; \frac{1}{2}, 0, \frac{1}{2}-z$, we obtain three possible generating complexes: $C_{c} I_{c} C_{c}$. This fact is not explicitly expressed in our symbolism, not even by the synonyms of the standard symbol of the LC.

In hemimorphic space group $\mathrm{P}_{2} \mathrm{me}$, positions $4(e) . m$. and $8(f) 1$ correspond to the LC symbols $\frac{1}{2} \frac{1}{2} J .2 P_{c} I 2 x[z]$ and $\ldots 2 P_{c} I 2 x 2 y[z]$. In both of these the distribution symmetry. .2 comes from $P 4_{2} / m m c$, position $8(q) m .$. , which defines the lattice complex $.2 P_{c} I 2 x 2 y$. Note that the $[z]$ operates on the whole complex, which can be shifted by any arbitrary $z$ value, and that we have $z=0$ in the characteristic space group. It follows that the 2 -fold axis (..2) must move with the complex.

In the orthorhombic system, we keep the holohedral space group in the same setting as in the 2nd edition of the International Tables (1952), but for hemimorphic space groups, we select a setting that retains the same systematic omissions and, therefore, shows the subgroup relations. Example: Space group $\mathrm{D}_{2 h}{ }^{5}$ is described in setting Emma. Space group $\mathrm{C}_{2 v}{ }^{4}$ is described in setting Pma2 in the International Tables, we keep this setting to facilitate comparisons, but we also give an alternate setting, $E_{m} 2 a$. 
SUBGROUP RELATIONS

(TABLES 39-43)

The consanguinity between space groups is determined by their subgroup relations. The description of the positions by means of lattice complexes provides a method to follow the effects of a certain subgroup relation on all the positions of a space group.

A complete discussion of the questions raised by subgroup relations for all the space groups would be an enormous task. We sha11, therefore, limit ourselves to a presentation of a few examples to show what types of relations are to be expected and the kind of results that can be obtained.

In a first example (Fig. Al), we consider the cubic asymmorphic ${ }^{1}$ space groups and show subgroup relations connection $\mathrm{R2}^{3}$ to its minimal supergroups, the latter to their minimal supergroups, and so on, up to the common symmorphic supergroup $\operatorname{Im} 3 \mathrm{~m}$. In a second example (Fig. A2), the filiation goes from $F d a d$, through its minimal supergroups and the supergroups of tine latter, to the common supergroup $P n 3 m$. In preparing these examples, we were able to take advantage of a pre-publication copy of an article on subgroup relations by Neubüser and Wondratschek (Priv. Comm., 1968), which was of great help to us. In the figures, a solid line connects a group to a subgroup of index 2, while a dashed line goes to a subgroup of index 4 (Fig. A1) or of index 3 (Fig. A2). Along the left margin, the scale indicates the multiples of the lowest order, 12, of group 22 3. A space-group symbol enclosed between parentheses and followed by a subscript 2 indicates that a block of eight cells, two in each of the three co-ordinate directions, is being

1 2. space group is called symmorphic if it contains a position whose site symmetry is the same as the point group isomorphic with the space group; hemisymmorphic, if it is of the second kind and contains a position whose site symmetry is the highest proper subgroup of the first kind of the isomorphic point group; asymmorphic, if neither of the above. Examples: Pm3m is symmorphic, Pn3n is hemisymmorphic, Pm3n is asymmorphic. Site symmetry $m 3 m$ is available in Pm3m, 432 in $P n 3 n$, but neither $m 3 m$ nor 432 is site symmetry in $P m 3 n$. considered.

If we compare corresponding positions in a space group and in one of its subgroups, we see that one of three situations may obtain:

1. The site symmetry of the position remains unchanged in the subgroup, but the multiplicity is decreased.

2. The multiplicity remains unchained, then it is the site symmetry that is decreased.

3. Both site symmetry and multiplicity can be decreased at the same time; this may happen when the subgroup is of index 4 or index greater than 4 .

These three rules apply vithout any modification to the description of positions by means of lattice complexes, whenever the positions have no degree of freedom. Examples: the relation between $\mathrm{I}_{2}$ and $\mathrm{P}_{2}$, which occur as $16(\alpha)$ in Ia $3 d$ and $8(\alpha)$ in Ia 3 respectively, is an example of Rule 1; the complex I, which occurs as $2(a)$ in $\mathrm{Im} 3 m$ and as $2(a)$ in $\mathrm{Em} 3 n$, illustrates Rule 2; and the relation between $W_{2}$ in $(\operatorname{Pm} 3 n)_{2}(c)$ and $V^{*}$ and $S^{*}$, which occur in $I a 3 d$ as $24(c)$ and $24(d)$, respectively, is explained by Rule 3 (Table 40).

For positions with degrees of freedom, on the other hand, the naming of the corresponding complex depends not only on the site symmetry, but also on the behavior of the generating (invariant) complex. Rule 1 can be subdivided into two cases:

1a. The site symmetry of the invariant position remains unchanged; then the invariant complex splits into complexes of lesser multiplicity. Example: $\overline{4} . . \gamma * * 2 x x x$ in Ia $3 d$ and $22 . . \gamma * 2 x x x$ in I4 32 (Table 39).

lb. The site symmetry of the invariant position does not remain uncilanged; then both the splitting number and the invariant complex change. Example: $\overline{4} . . Y * \star 2 x x x$ in Ia $3 d$ and $\left.\overline{4} . . I_{2}{ }^{* *}\right] \times x x$ in I $43 d$ (Table 39).

Rule 2 gives rise to three cases.

2a. The position and the invariant generating complex suffer a decrease in site symmetry without any change in the naming of the generating complex; then the variant lattice complex remains the same. Example: $16 z$ in $I m 3 m, 12(e) 4 m . m$, remains $16 z$ in $\operatorname{Pm} 3 n, 12(f) m m 2 .$. (Table 40).

2b. In the naming of the position, the invariant 
generating complex remains unchanged, but the point complex changes; then some distribution symmetry may have to be specified. Example: $112 x x$ in $\operatorname{Im} 3 m(h)$ and .2 I6z2x in $\operatorname{Pm} 3 n(k)$ (Table 40).

$2 c$. The change in site symmetry requires a completely different generating complex. Example:

$\overline{4} a . . Y * * 3 x \bar{x}$ in $\operatorname{Ia} 3 d(g)$ and $22 \ldots P_{2} 6 x y z$ in $\operatorname{Ia} 3(e)$ (Table 40).

Relationships are shown between asymmorphic cubic body-centered space groups, which have the same cell: $\left.I a 3 d, I \overline{4} 3 d, I 4\}^{32}, I a 3, I 2\right\rceil^{3}$ (Table 39). The series $\operatorname{Im} 3 m, \operatorname{Rm} 3 n, I a 3 d, I a 3, P a 3, P 2,3$ is taken from Fig. Al and the subgroup relations are explained in Table
40. Here a set of points in the supergroup, Ia3d or $(\operatorname{Im} 3 m)_{2}$, is followed in all the subgroups until the general position is reached in a subgroup. In Table 41 the positions in space group $P a 3$ are related to those in the minimal supergroups $I a 3$ and $\mathrm{Fm} 3$. In Table 42 two space groups, $F 4{ }_{1} 32$ and $I 4{ }_{1} 32$, have a common subgroup $\mathrm{P}_{3} 32$; the solid lines show where the special positions of $\left.F^{4}\right]^{32}$ and $\left.I^{4}\right]^{32}$ are to be found in the subgroup. Finally, Table 43 shows the relationships between positions in different crystal systems, illustrated by the space groups $F d 3 m, F d 3, I 4{ }_{1} /$ and and Fddd. 
Fig. Al. Subgroup relations in cubic asymmorphic space groups;

$m 3 m$

$\overline{4} 3 m$

432

m3

23

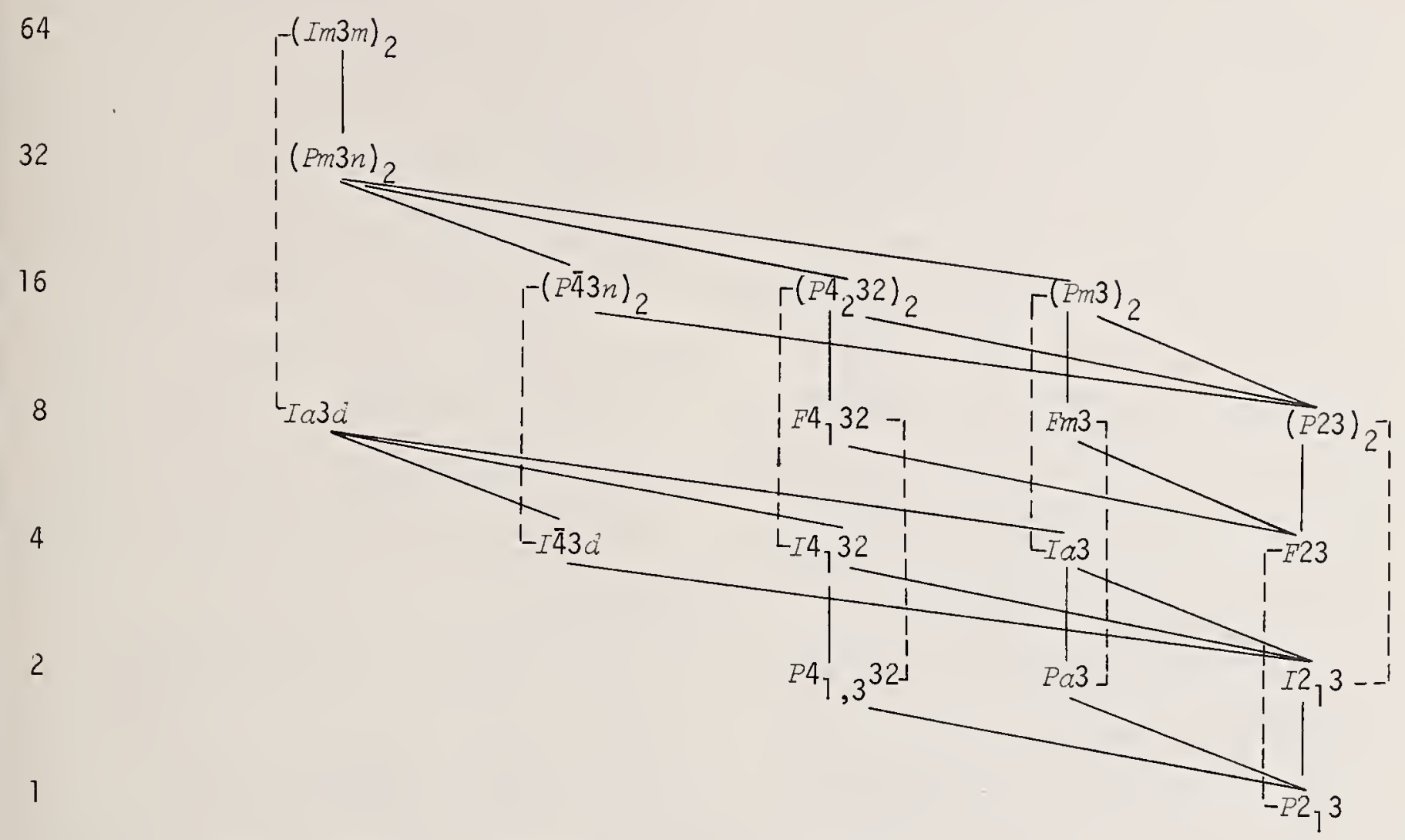

Fig. A2. Subgroup relations in space groups of different systems.

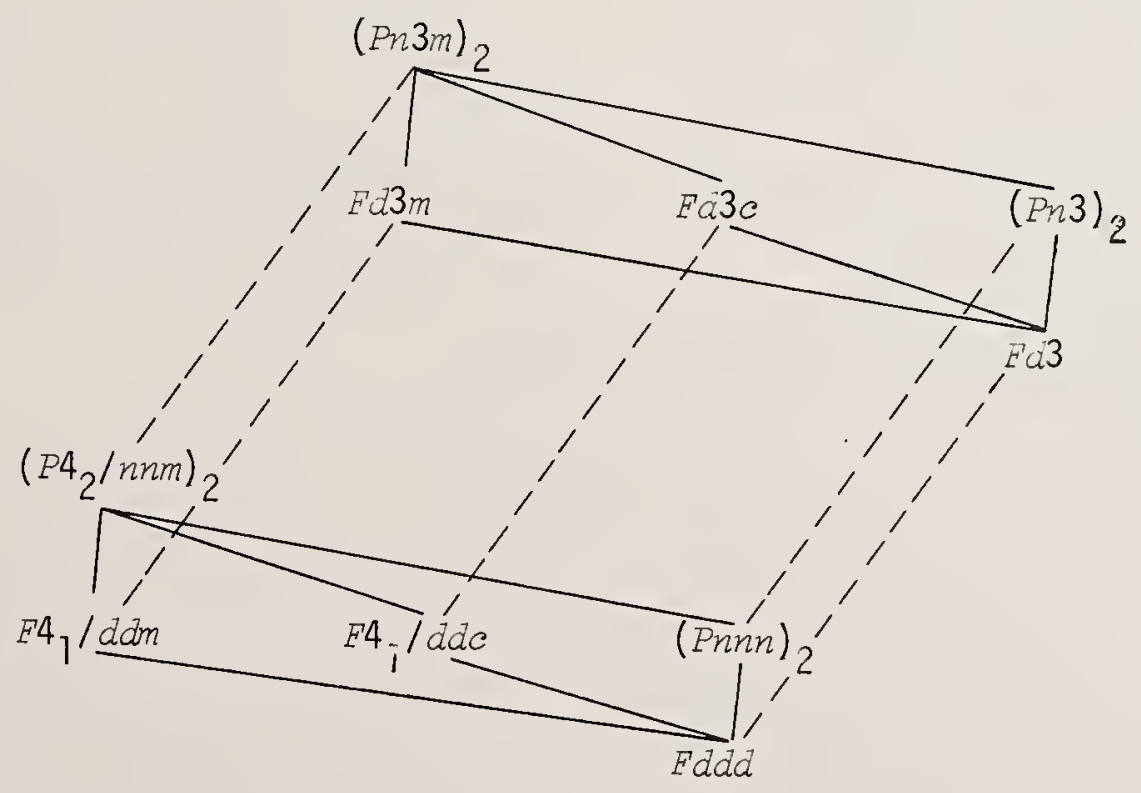


Belov, N.V., N.N. Neronova, J.D.H. Donnay and G. Donnay (1960) Tables of magnetic space groups. Acta Cryst. $13,1085$.

Belov, N.V., N.N. Neronova, J.D.H. Donnay and G. Donnay (1962) Tables of magnetic space groups. II. Special positions. Jour. Phys. Soc. Japan 17 (B-II), 332-335.

Boldyrev, A.K. (1925) Die vom Fedorow-Institut angenommene kristallographische Nomenklatur. Z. Krist. $62,145-150$.

Burzlaff, H., W. Fischer and E. Hellner (1968) Die Gitterkomplexe der Ebenengruppen. Acta Cryst. A24, 57-67.

Burzlaff, H., W. Fischer and E. Hellner (1969) Bemerkungen zu "Die Gitterkomplexe der Ebenengruppen" (Acta Cryst. A24, 57, 1968) und "Kreispackungsbedingungen in der Ebene" (Acta Cryst. A24, 67, 1968). Acta Cryst. A25, 710-711.

Donnay, J.D.H. (1947a) Hexagonal four-index symbols. Amer. Mineral. 32, 52-58.

Donnay, J.D.H. (1947b) The superabundant index in the hexagonal Bravais symbol. Amer. Mineral. 32, 477-478.

Donnay, J.D.H. (1969) Symbolism of rhombohedral space groups in Miller axes. Acta Cryst.A25, 715-716.

Donnay, J.D.H., E. Hellner and A. Niggli (1964) Coordination polyhedra. Z. Krist. 120, 364-374. Donnay, J.D.H., E. Hellner and A. Niggli (1966) Symbolism for lattice complexes revised by a Kiel Symposium. Z. Krist. 123, 255-262.

Fischer, W. (1968) Kreispackungsbedingungen in der Ebene. Acta Cryst. A24, 67-81.

Hellner, E. (1965) Descriptive symbols for crystalstructure types and homeotypes based on lattice complexes. Acta Cryst. 19, 703-712.

Hermann, C. (1935) Gitterkomplexe. Int. Tab. zur Bestimmung von Kristallstrukturen, vol. 1, chapters VI and VII.
Hermann, C. (1953) Paper presented at the Deutsche Mineralogische Gesellschaft, Sektion für Kristallkunde, Erlangen meeting.

Hermann, C. (1960) Zur Nomenklatur der Gitterkomplexe. Z. Krist., 113, 142-154.

Internationale Tabellen zur Bestimmung von Kristallstrukturen (1935) 2 vols. Berlin, Borntraeger. International Tables for X-ray Crystallography, vol. 1 (1952, 1965, 1969). Birmingham, England, The Kynoch Press.

Loeb, A.L. (1967a) Crystal algebra of cubic lattice complexes. Tech. Rept. 130 , Ledgemont Laboratory, Lexington, Mass.

Loeb, A.L. (1967b) Domains, interstices and lattice complexes: derivatives from the b.c.c. lattice. Tecn. Rept. 133, Ledgemont Laboratory, Lexington, Mass.

Loeb, A.L. (1970) A systemative survey of cubic crystal structures. J. Solid State Chem. 1, 237-267.

Niggli, P. (1919) Geometrische Kristallographie des Diskontinuums. Leipzig, Bornträger.

Niggli, P. (1920) Lehrbuch der Mineralogie. Berlin, Borntraeger. (1st ed., pp. 125 et ss.); 2nd ed., 1924, pp. 168 et ss.; 3rd ed., 1941,pp.275 et ss. Niggli, P. (1928) Kristallographische und strukturtheoretische Grundbegriffe. Leipzig, Akad. Verlagsges.

Smirnova, N.L. (1966) An analytical method of representation of inorganic crystal structures. Acta Cryst. 21, A33.

Smirnova, N.L., and L.I. Poteshnova (1966) Vestnik Moskovskogo Univ., Ser. Geol. No. 6.

Weber, L. (1922) Das viergliedrige Zonensymbol des hexagonalen Systems. Z. Krist. 57, 200-203. 
FIGURES

$501-5630-73-3$ 
Figs. 1-47. Site sets in stereographic projection.

Name of site set as co-ordination polyhedron and of its dual as crystal form.

Fig. 1. Point. Monohedron.

2. Line segment (through 0). Pinacoid.

3. Line segment (off 0 ). Dihedron.

4. Trigon (through 0 ). Trigonal prism.

5. Trigon (off 0). Trigonal pyramid.

6. Rectangle (through 0). Rhombic prism.

7. Rectangle (off 0). Rhombic pyramid.

8. Tetragon (through 0). Tetragonal prism.

9. Tetragon (off 0 ). Tetragonal pyramid.

10. Rhombic tetrahedron. Rhombic tetrahedron.

11. Tetragonal tetrahedron. Tetragonal tetrahedron.

12. Regular tetrahedron. Regular tetrahedron.

13. Truncated trigon (through 0 ). Ditrigonal prism.

14. Truncated trigon (off 0 ). Ditrigonal pyramid.

15. Hexagon (through 0 ). Hexagonal prism.

16. Hexagon (off 0 ). Hexagonal pyramid. 

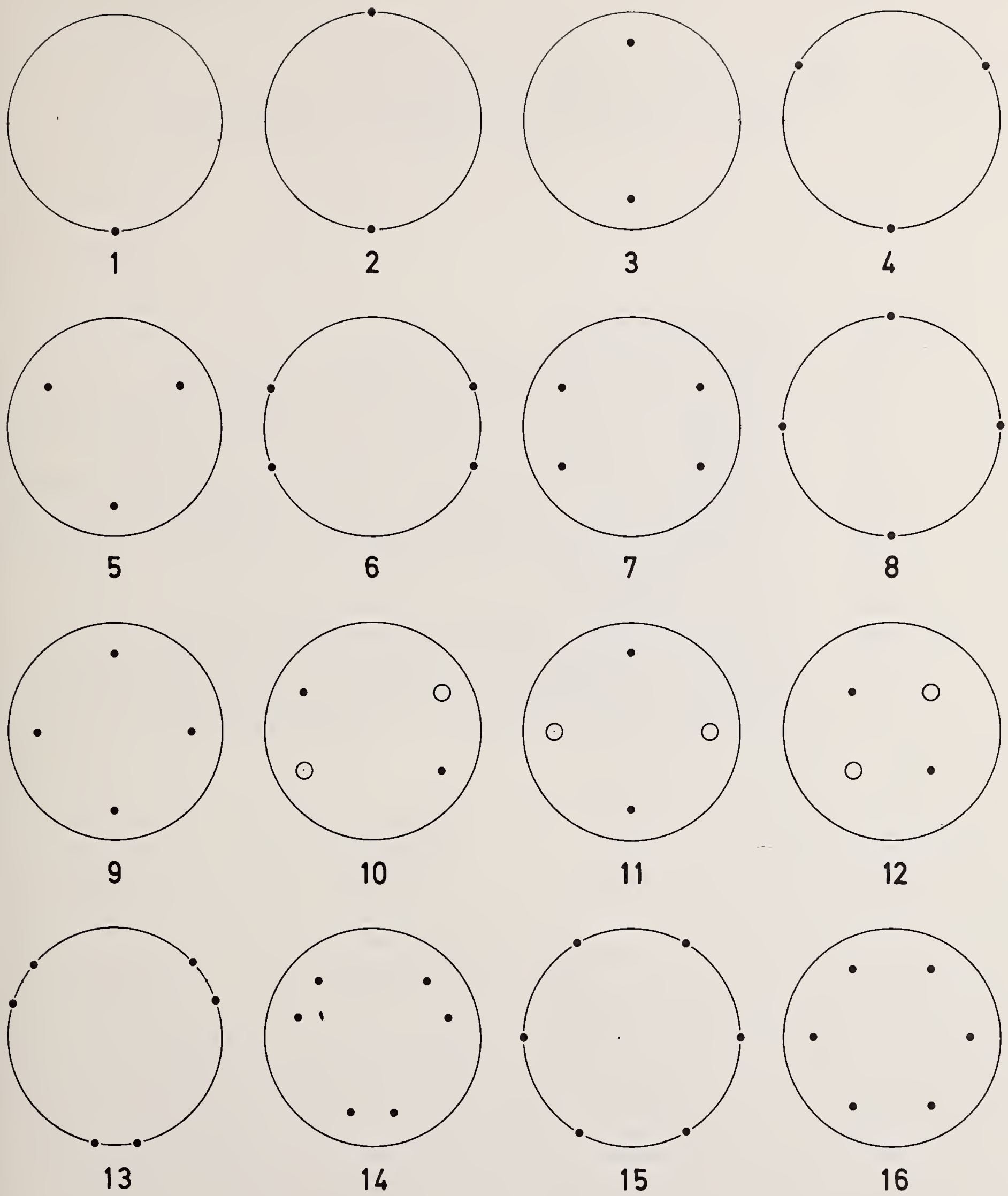
Figs. 1-47. Site sets in stereographic projection.

Name of site set as co-ordination polyhedron and of its dual as crystal form.

Fig. 17. Octahedron. Cube.

18. Trigonal antiprism. Rhombohedron.

19. Trigonal prism \& pinacoid. Trigonal dipyramid.

20. Trigonal trapezohedron \& pinacoid. Trigonal trapezohedron.

21. Truncated tetragon (through 0 ). Ditetragonal prism.

22. Truncated tetragon (off 0 ). Ditetragonal pyramid.

23. Tetragonal tetrahedron \& pinacoid. Tetragonal scalenohedron.

24. Tetragonal prism \& pinacoid. Tetragonal dipyramid.

25. Tetragonal trapezohedron \& pinacoid. Tetragonal trapezohedron.

26. Rectangular parallelepiped. Rhombic dipyramid.

27. Cube. Octahedron.

28. Truncated hexagon (through 0). Dihexagonal prism.

29. Truncated hexagon (off 0 ). Dihexagonal pyramid.

30. Pinacoid \& rhombohedron. Hexagonal scalenohedron.

31. Truncated trigonal prism \& pinacoid. Ditrigonal dipyramid.

32. Hexagonal prism \& pinacoid. Hexagonal dipyramid. 

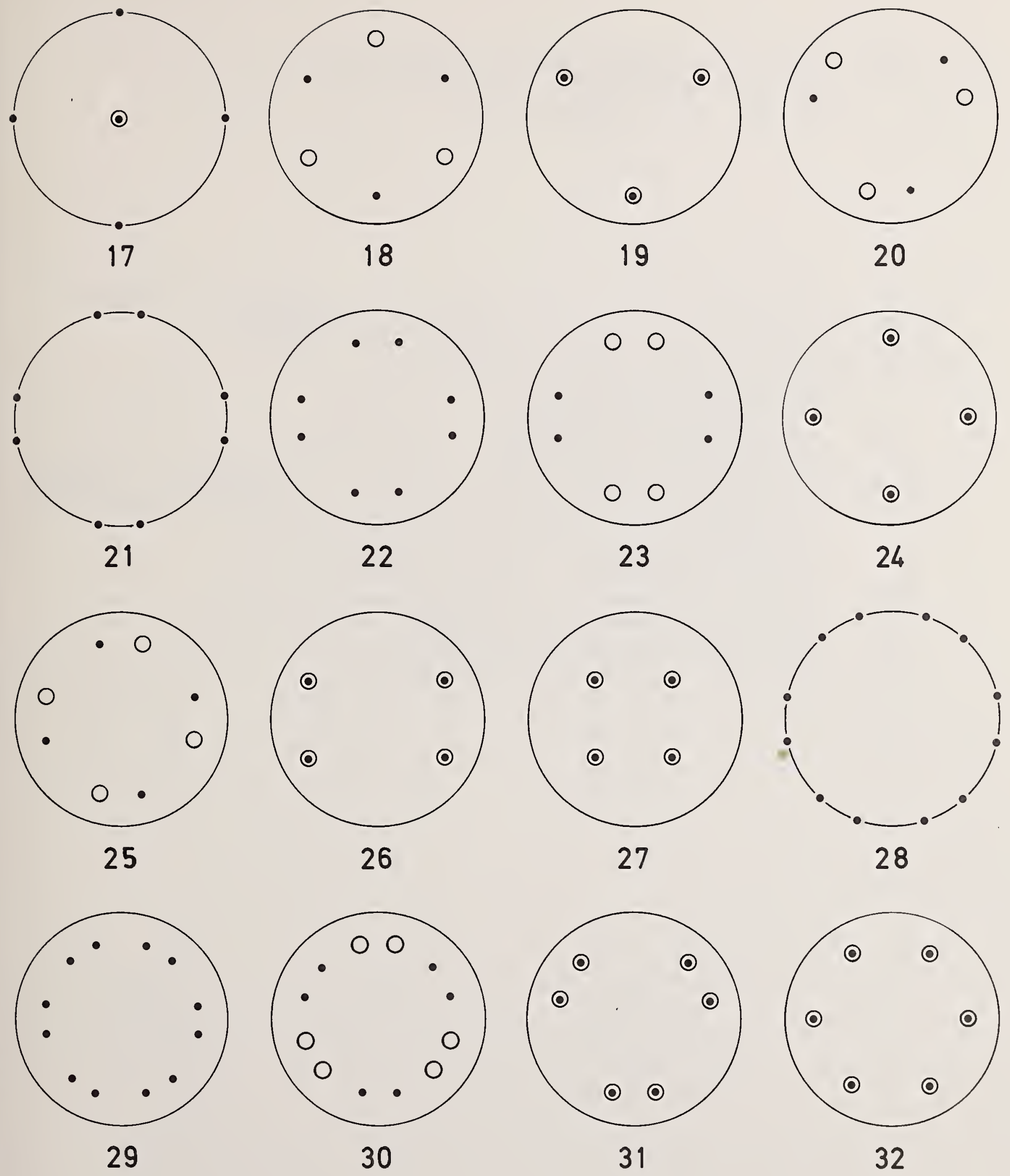
Figs. 1-47. Site sets in stereographic projection. Name of site sets as co-ordination polyhedron and of its dual as crystal form.

Fig. 33. Hexagonal trapezohedron \& pinacoid. Hexagonal trapezohedron.

34. Truncated tetrahedron. Trigon-tritetrahedron.

35. Cube \& 2 tetrahedra. Tetragon-tritetrahedron.

36. Cuboctahedron. Rhomb-dodecahedron.

37. Pentagon-tritetrahedron \& 2 tetrahedra.

Pentagon-tritetrahedron.

38. Dihexahedron \& octahedron. Dihexahedron.

39. Truncated tetragonal prism \& pinacoid. Ditetragonal dipyramid.

40. Truncated hexagonal prism \& pinacoid. Dihexagonal dipyramid.

41. Truncated cube. Trigon-trioctahedron.

42. Rhombicuboctahedron. Tetragon-trioctahedron.

43. Cube \& 2 tetrahedra. Hexatetrahedron.

44. Truncated octahedron. Tetrahexahedron.

45. Cube \& octahedron \& dihexahedron. Didodecahedron.

46. Snub cube. Pentagon-trioctahedron.

47. Truncated cuboctahedron. Hexaoctahedron. 

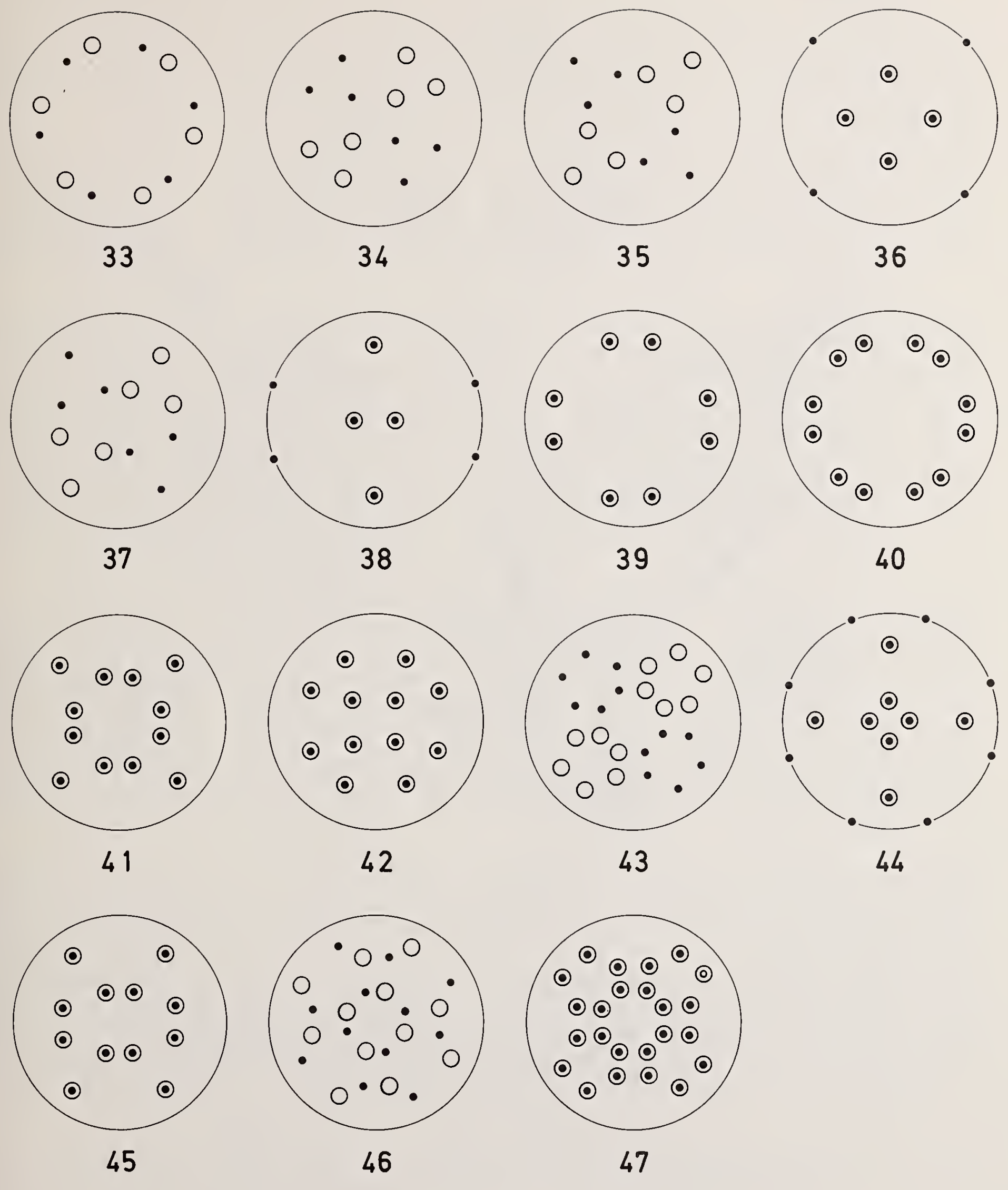


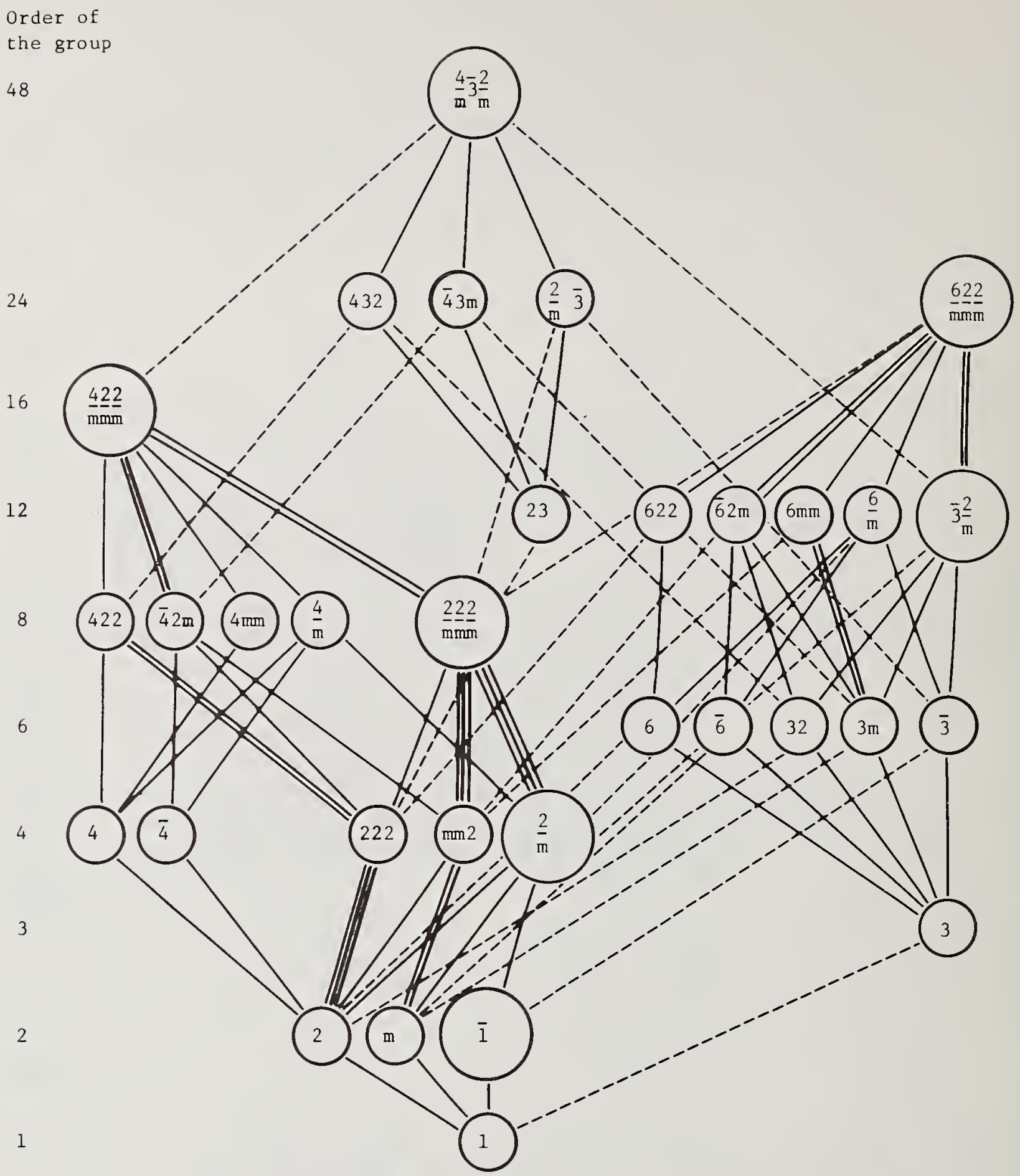

Fig. 48. The point group at the lower end of a connecting line is a subgroup of that at the upper end. Double (or triple) lines mean that the lower group is subgroup of the upper one in two (or three) non-equivalent orientations. Dashed lines show subgroups of index $>2$.

(After C. Hermann, Int. Tab., vol. I, p. 49, 1935.) 

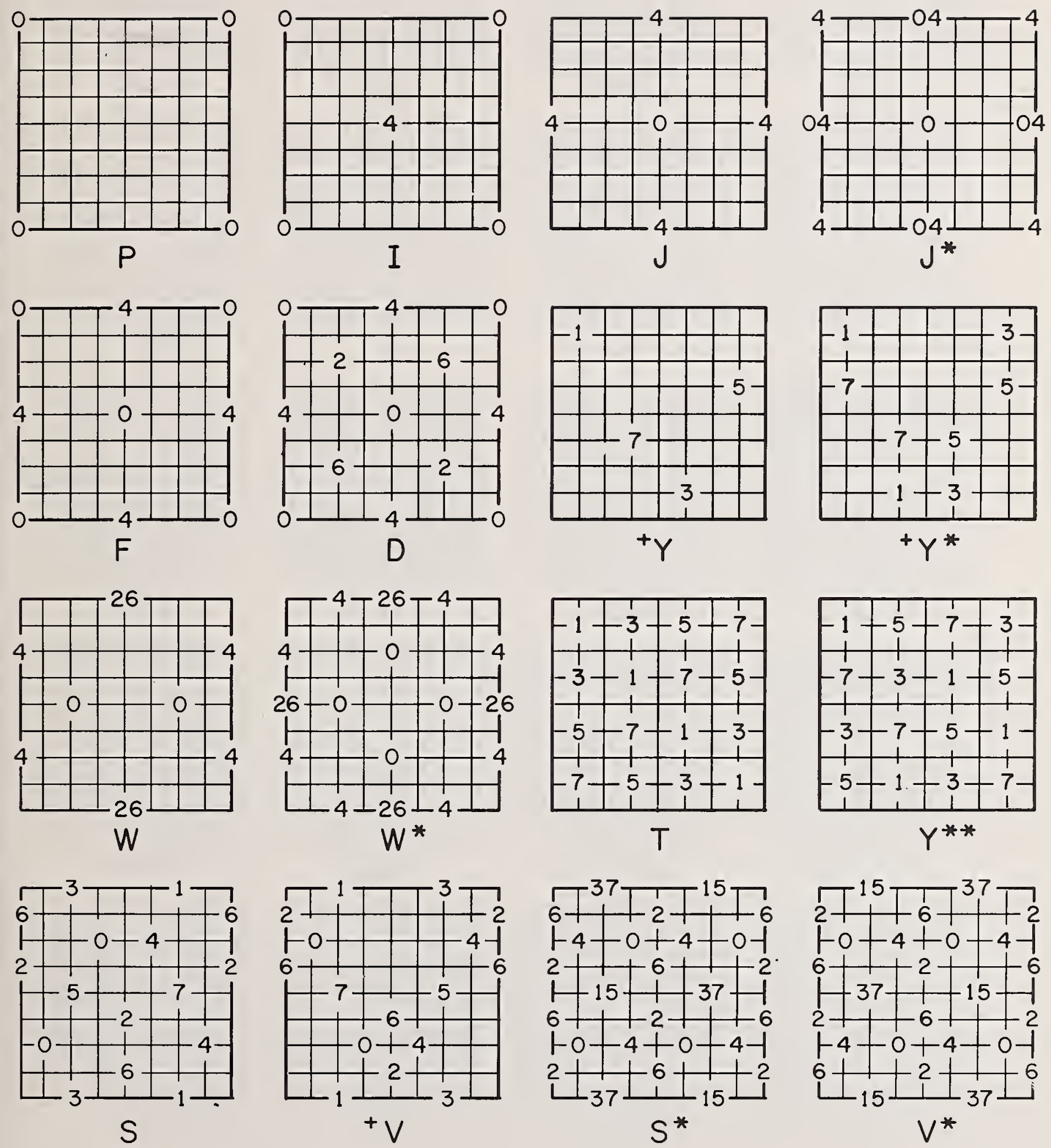

Fig. 49.

The 16 cubic invariant lattice complexes in standard orientation. Heights in eighths of cell edge. 

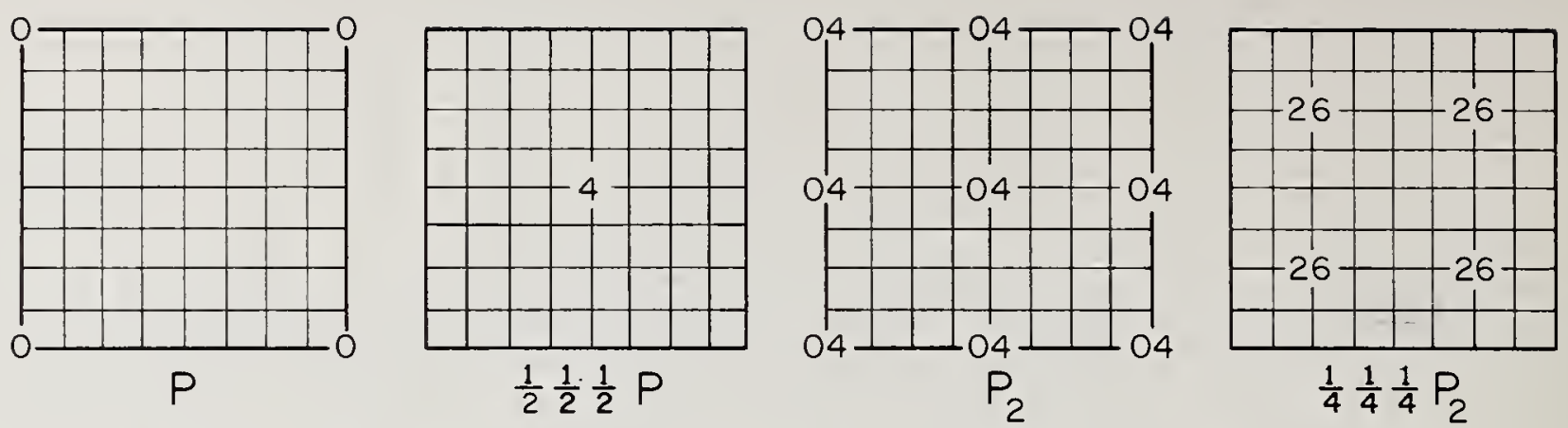

$\frac{1}{2} \frac{1}{2} \frac{1}{2} P$
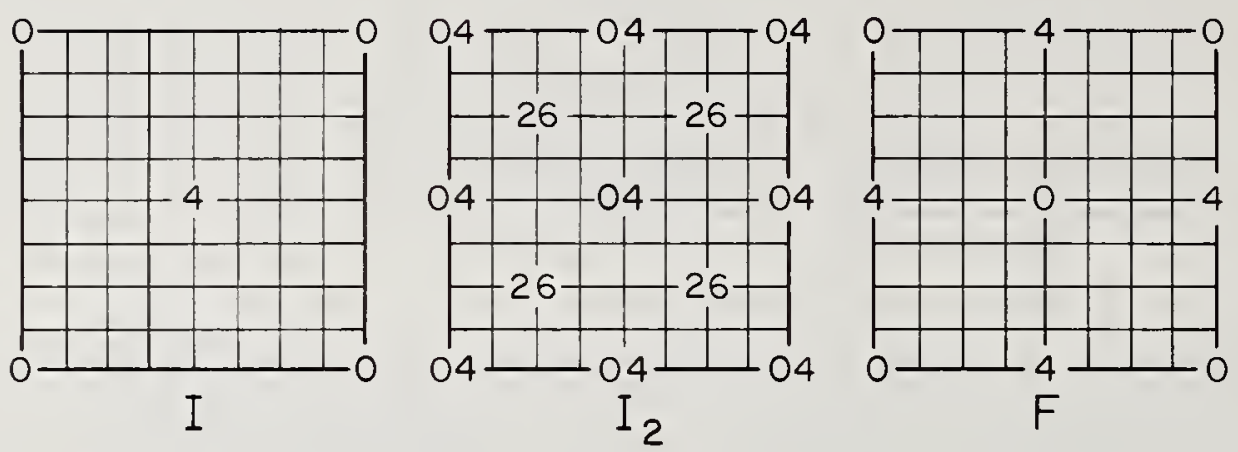

$\frac{1}{4} \frac{1}{4} \frac{1}{4} P_{2}$

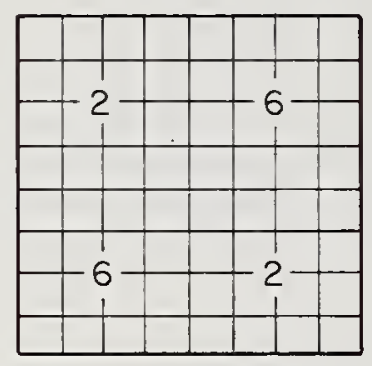

$\frac{1}{4} \frac{1}{4} \frac{1}{4} \mathrm{~F}$

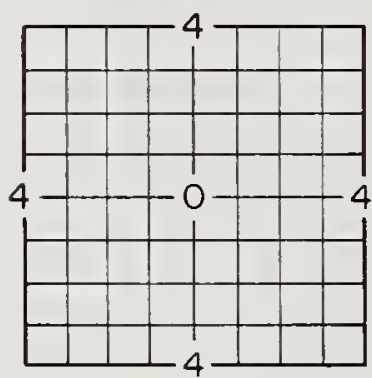

J

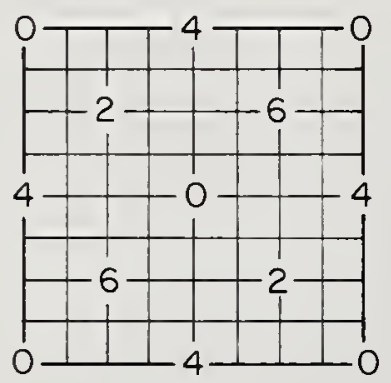

D

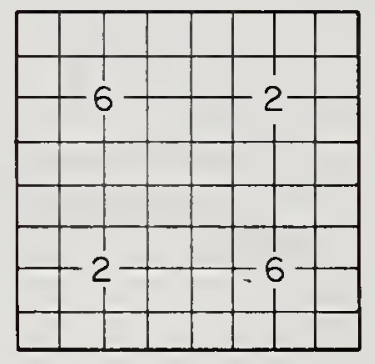

$\frac{3}{4} \frac{3}{4} \frac{3}{4} F$

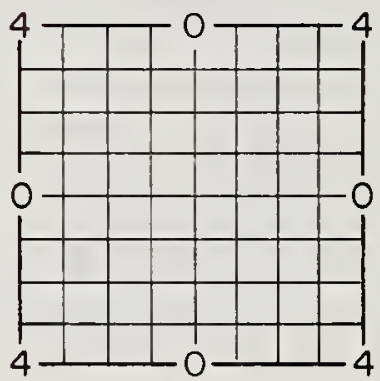

$\frac{1}{2} \frac{1}{2} \frac{1}{2} \mathrm{~J}$

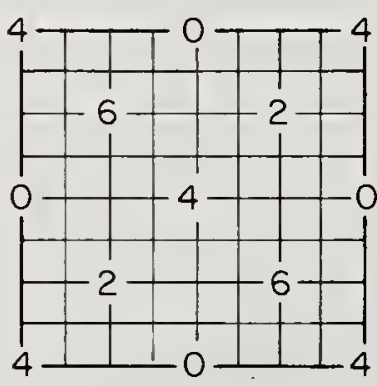

$\frac{1}{2} \frac{1}{2} \frac{1}{2} D$

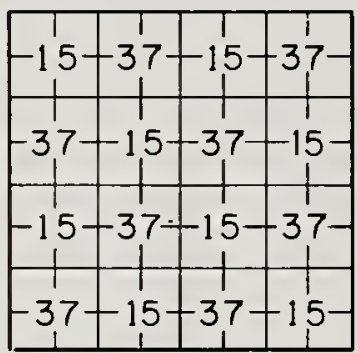

$\frac{1}{8} \frac{1}{8} \frac{1}{8} F_{2}$

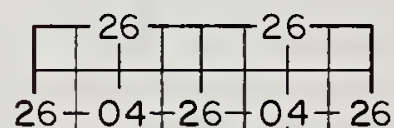

$26-04-26-04-26$

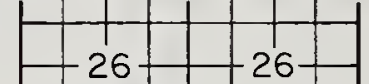

$26-04-26-04-26$

\begin{tabular}{llllll}
$y_{26}$ & & & 1 & \\
\hline
\end{tabular}

$\mathrm{J}_{2}$

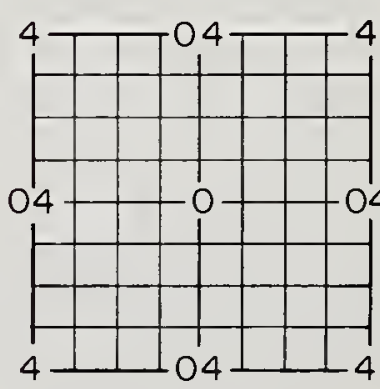

$J^{*}$

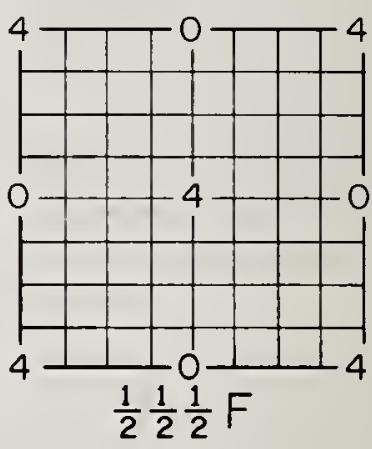

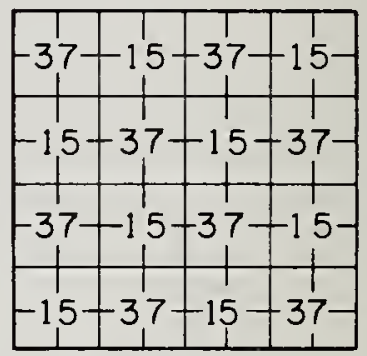

$\frac{3}{8} \frac{3}{8} \frac{3}{8} F_{2}$

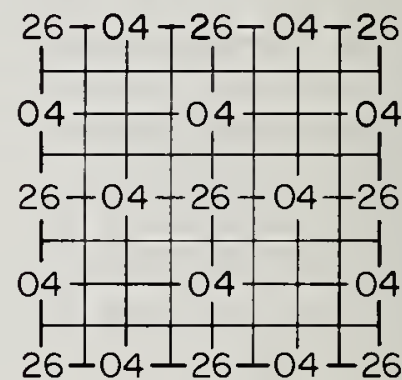
$\frac{1}{4} \frac{1}{4} \frac{1}{4} \mathrm{~J}_{2}$

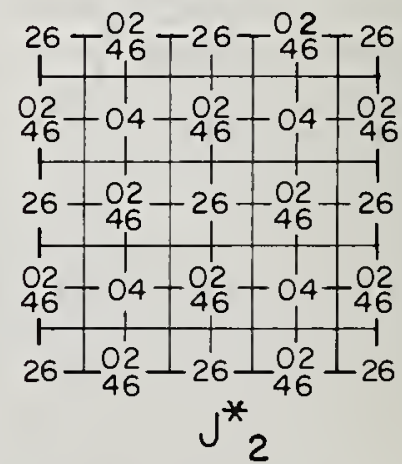

Fig. 50. Cubic invariant lattice complexes and representations. Heights in eighths of cell edge. 

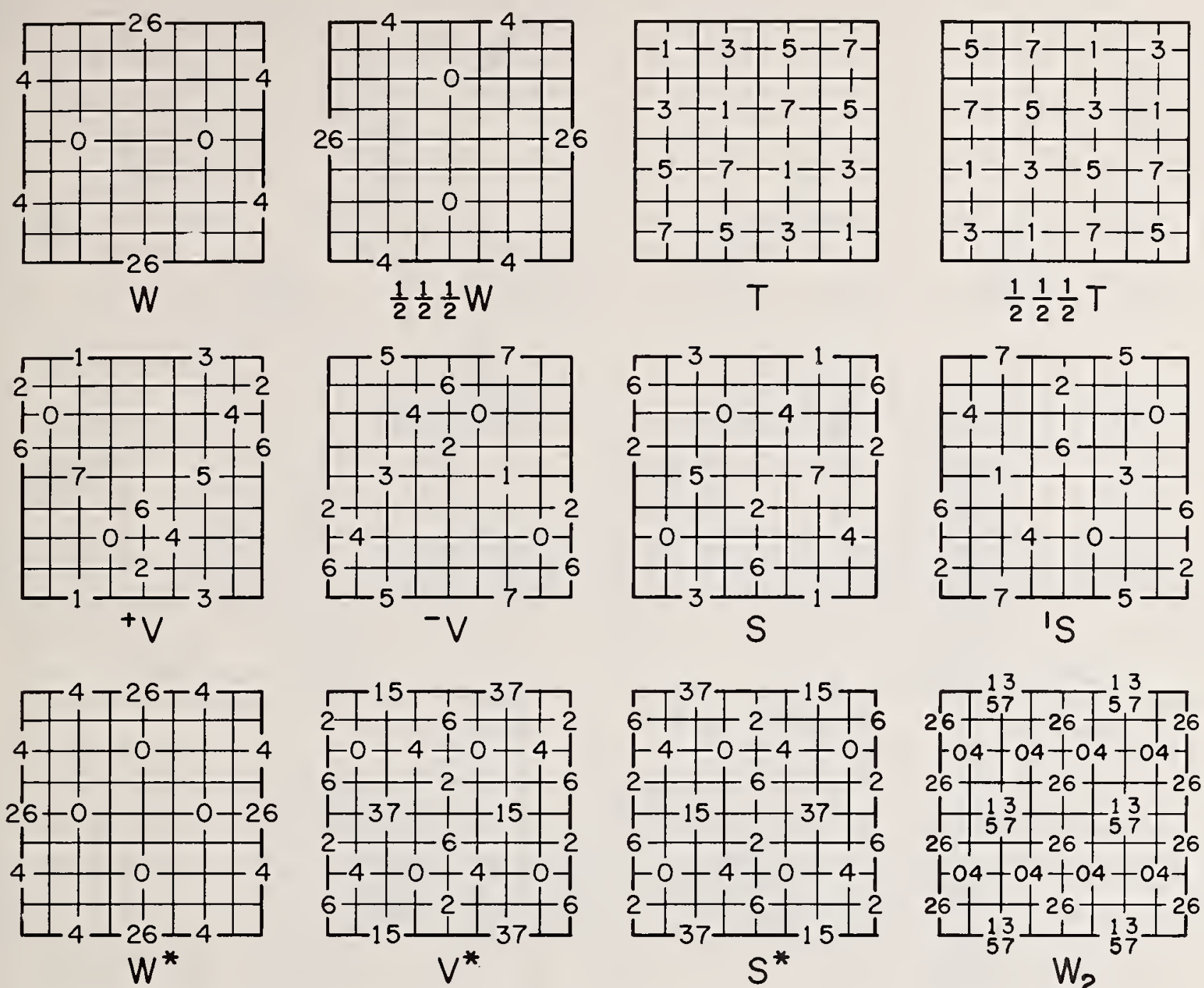

$6+37+2+15+6$

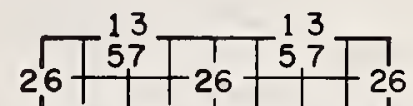
$14-0+4+0-1$-04-04+04-04-1

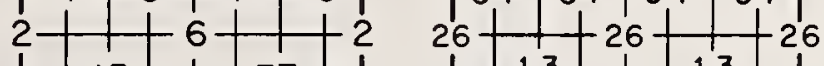

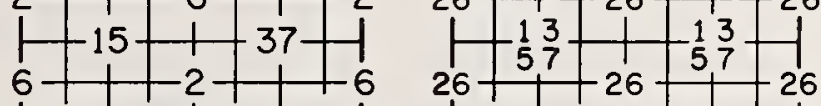
$10-4+0-4-\quad$ Ho4-04 $+04-04-1$ $2+\frac{6}{1}+6+I_{15}+2$ $S^{*}$
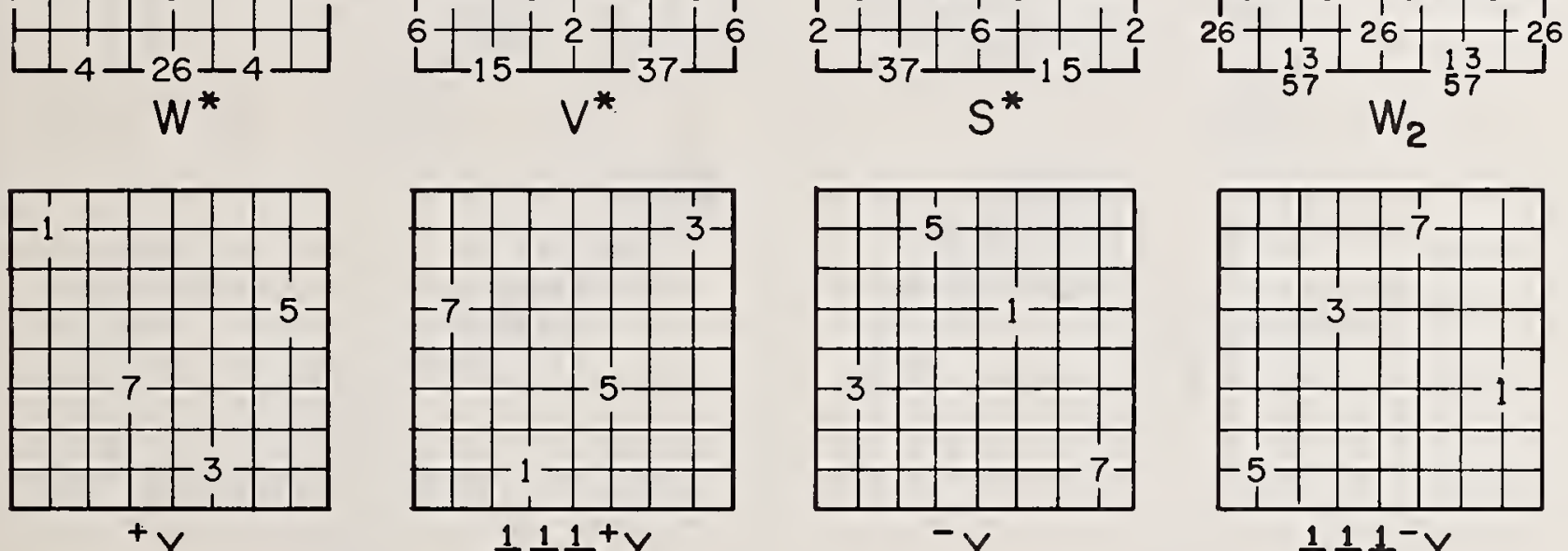

$$
\frac{1}{2} \frac{1}{2}^{+}{ }^{+} Y
$$
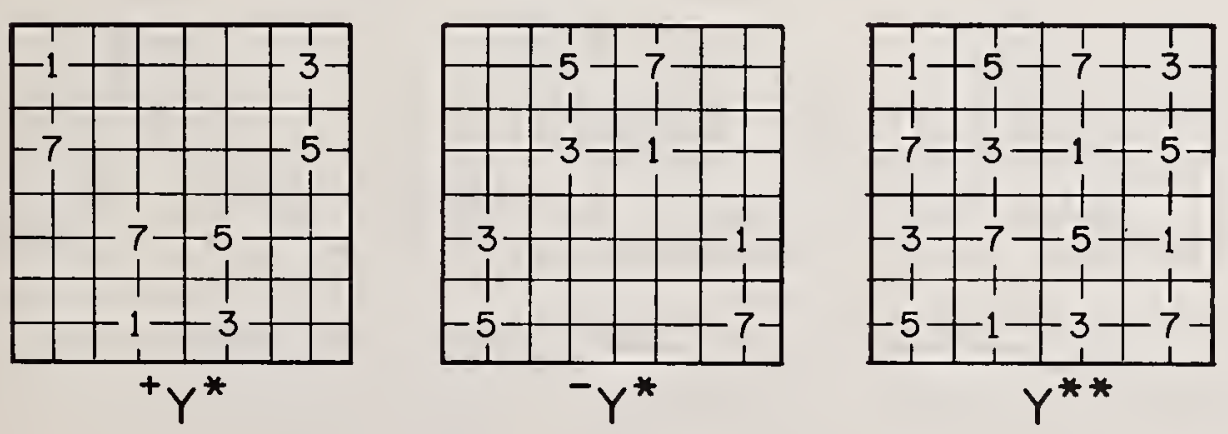

\section{$\frac{1}{2} \frac{1}{2} \frac{1}{2}^{-} Y$}

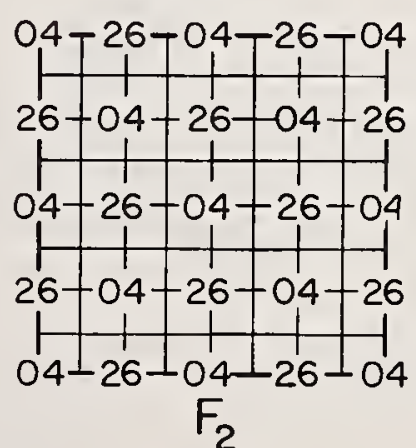

Fig. 51

Cubic invariant lattice complexes and representations (continued). Heights in eighths of cell edge. 

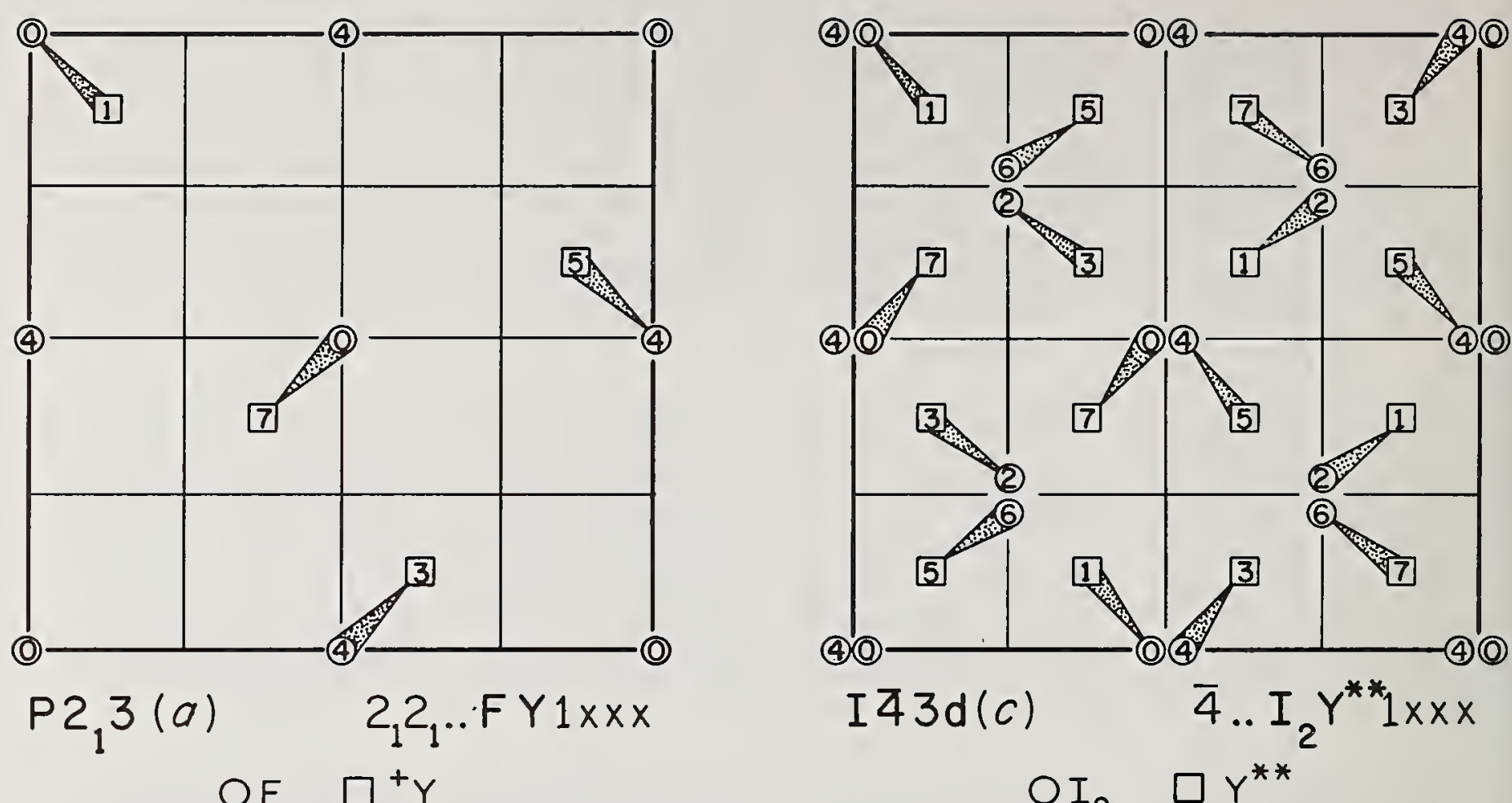

$\mathrm{OI}_{2} \square Y^{* *}$
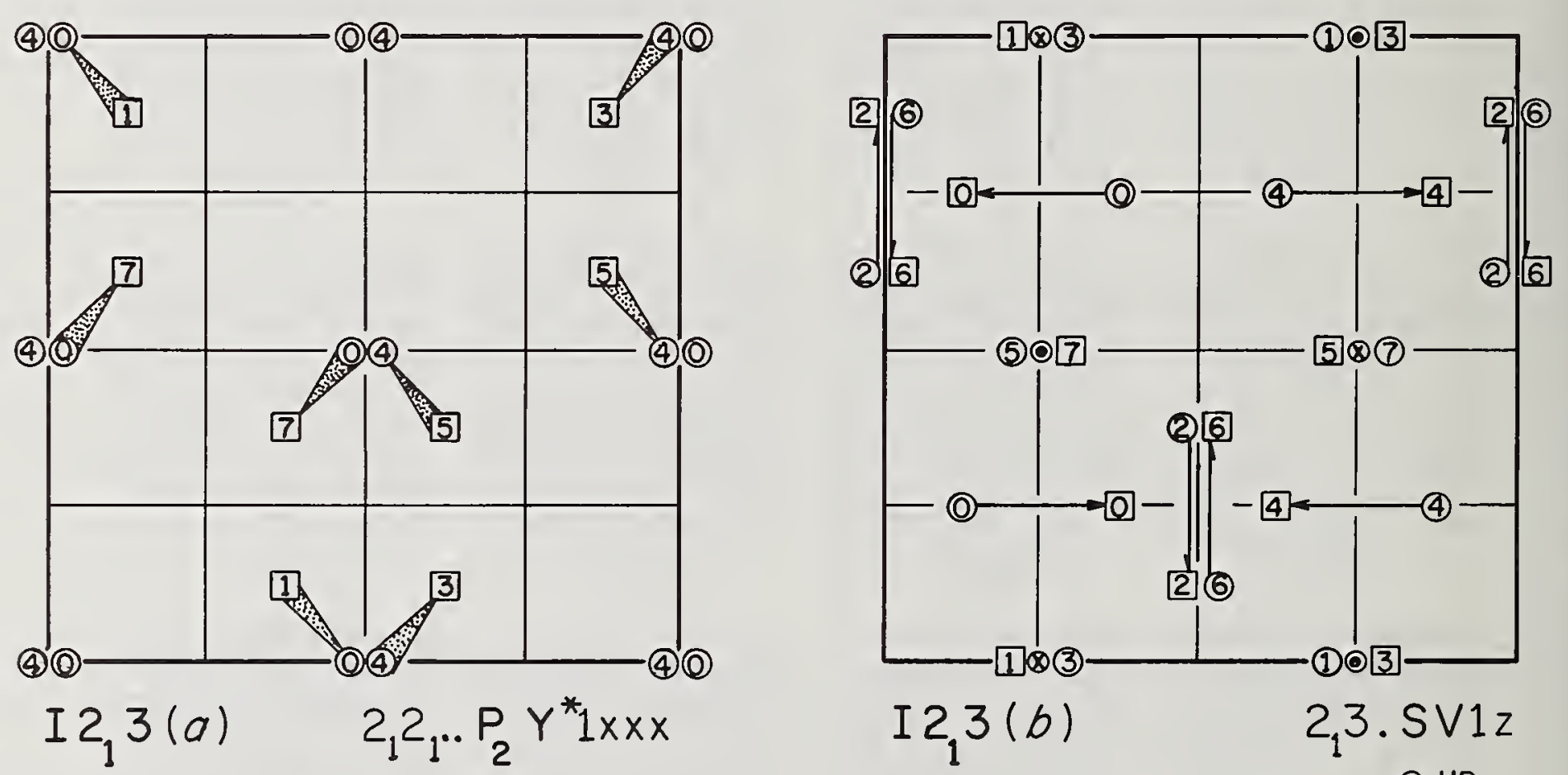

$I 2_{1} 3(a) \quad 2_{1} 2_{1} \ldots P_{2} Y^{*} 1 \times x x$

$O P_{2} \quad \square Y^{*}$

os $\square^{+} v$

U DOWN

Fig. 52. Cubic univariant Weissenberg complexes in standard representation and characteristic space group.

Heights in eighths of cell edge. (See section 7.31 , p. 19.) 


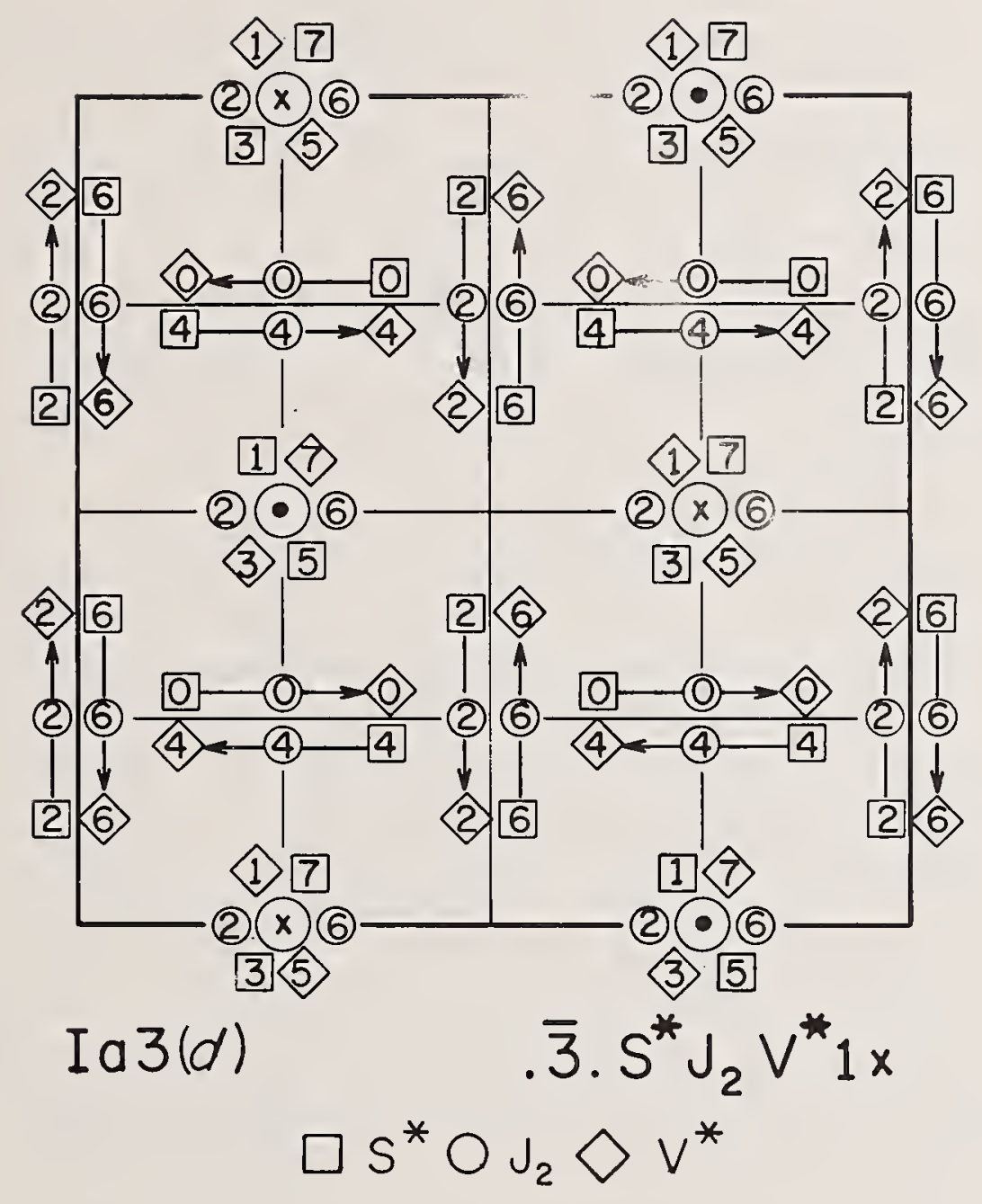

Fig. 53. Cubic univariant Weissenberg complexes in standard representation and characteristic space group. Heights in eighths of cell edge. (See Section 7.31, p. 19.) (continued). 

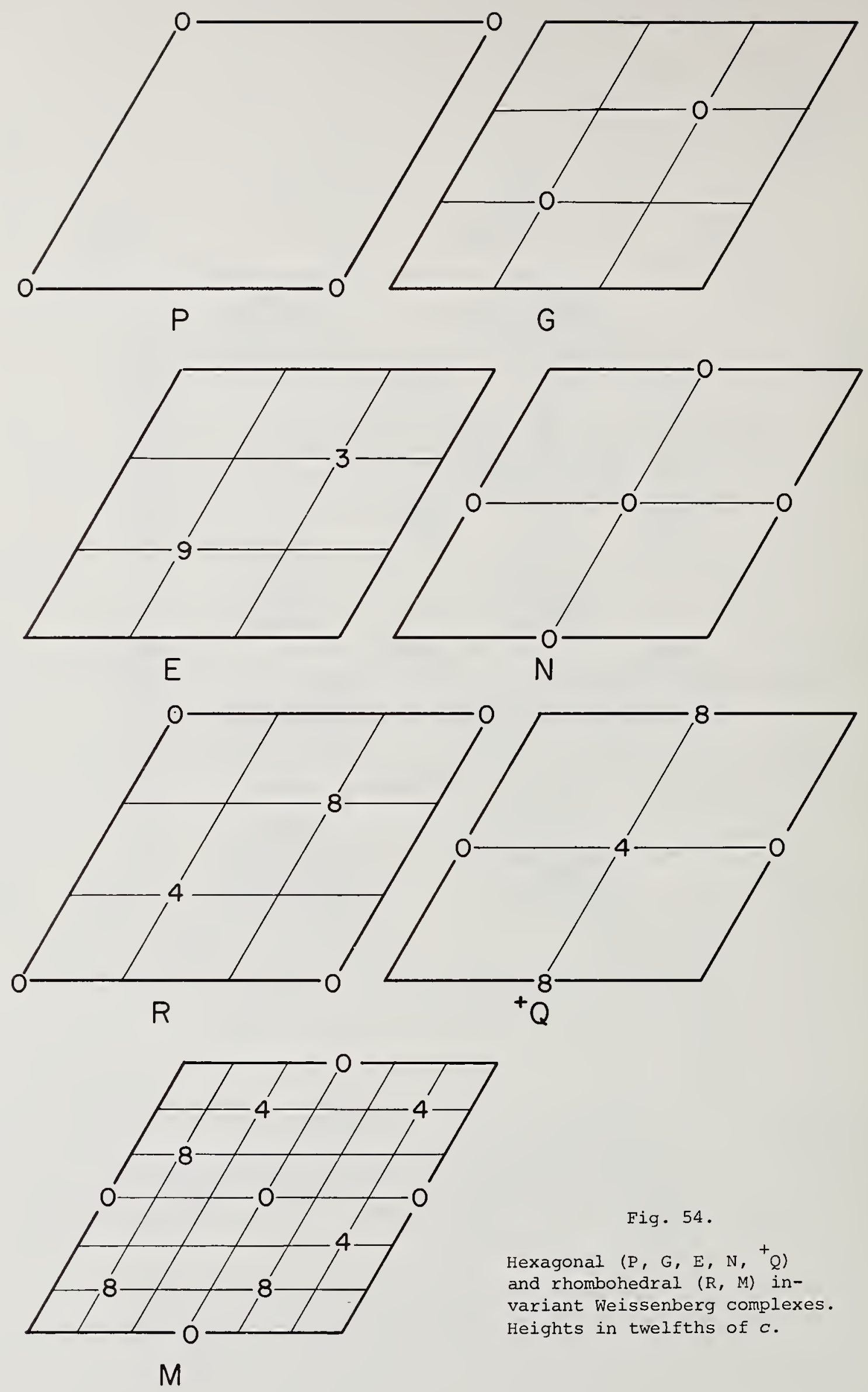

Fig. 54 .

Hexagonal (P, G, E, N, ${ }^{+} \mathrm{Q}$ ) and rhombohedral ( $R, M$ ) invariant Weissenberg complexes. Heights in twelfths of $c$. 

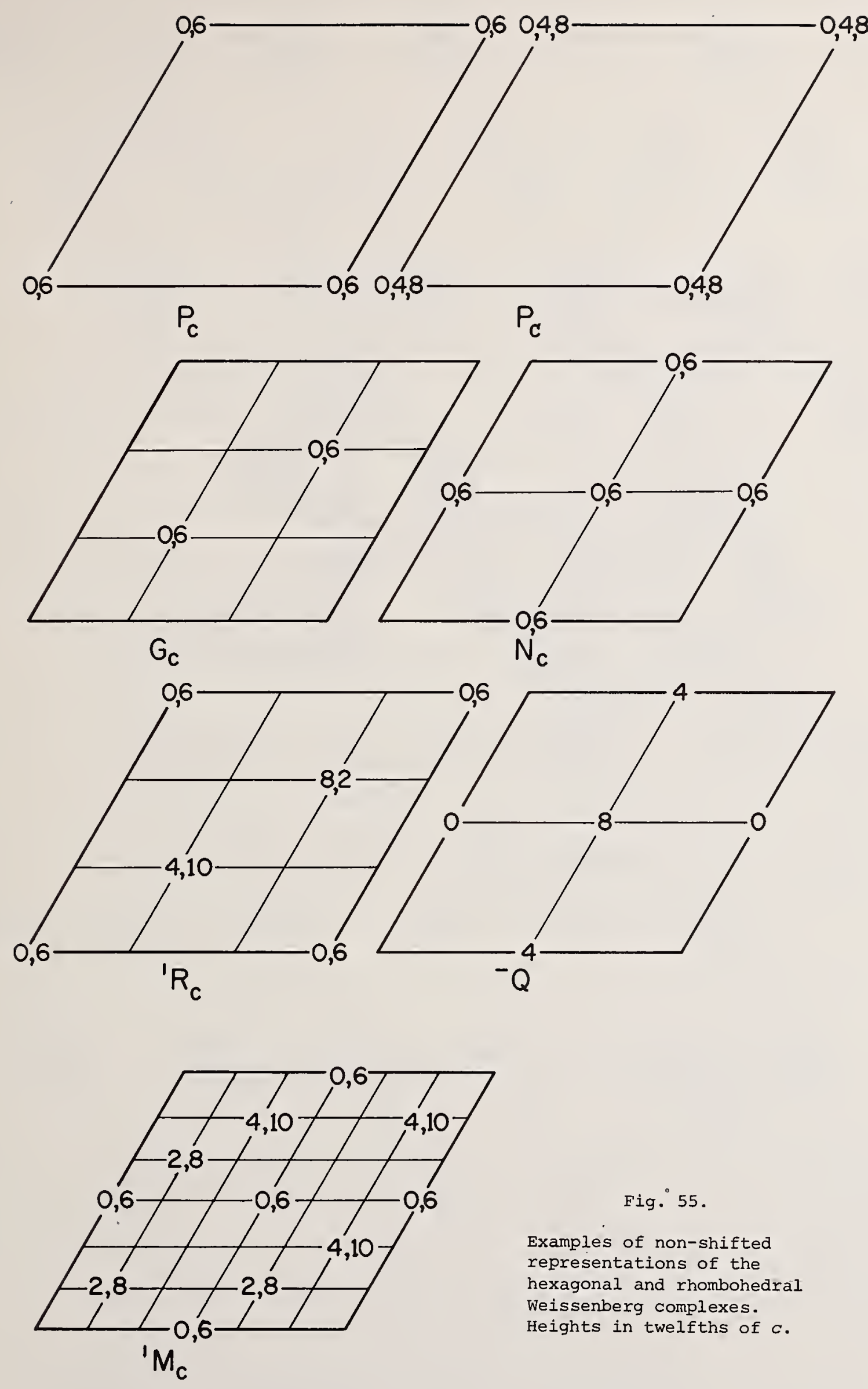

Fig. 55.

Examples of non-shifted representations of the hexagonal and rhombohedral Weissenberg complexes. Heights in twelfths of $c$. 


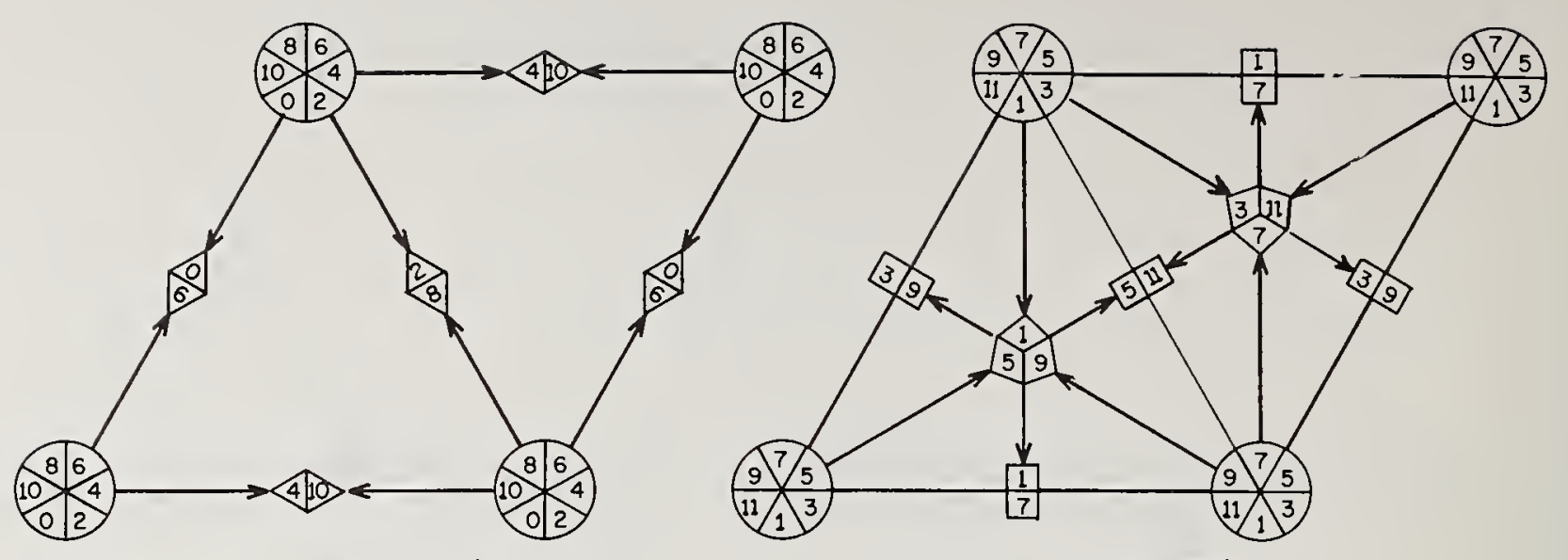

$$
P 6,22 \text { (a) } 3,2 P_{C c}{ }^{+} Q_{c} 1 x
$$
$\notin \quad P_{C} \triangleright{ }^{+} Q_{c}$

$P 6,22(b) 3,2 \cdot P_{c c} E_{c}^{+} Q_{c} 1 \times \bar{x}$
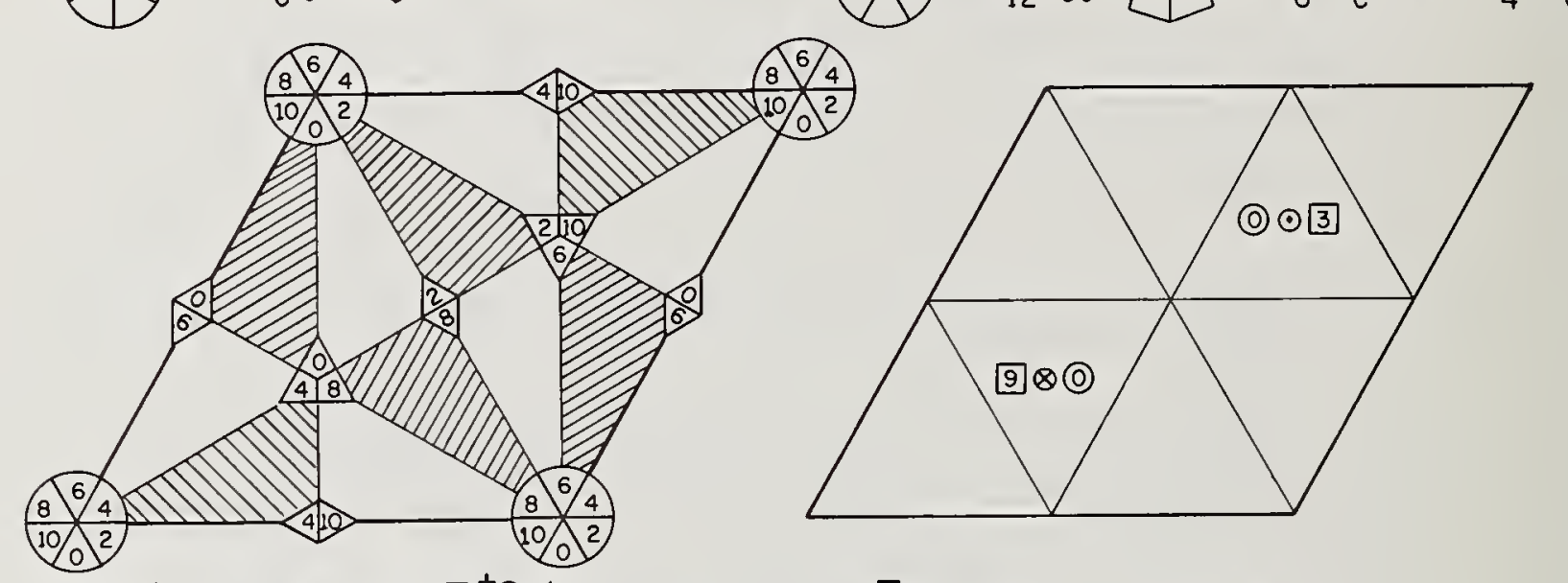

$P 6_{1}(a) \quad 3,2_{1} \ldots P_{C c} E_{c}^{+} Q_{c} 1 x y$

$\otimes P_{c c} \quad A O O_{12} E_{c} \theta^{+} Q_{c}$

$\bigotimes 00 \frac{11}{12} P_{C_{c}} \otimes 00 \frac{1}{6} E_{c} \square 00 \frac{1}{4}^{+} Q_{c}$

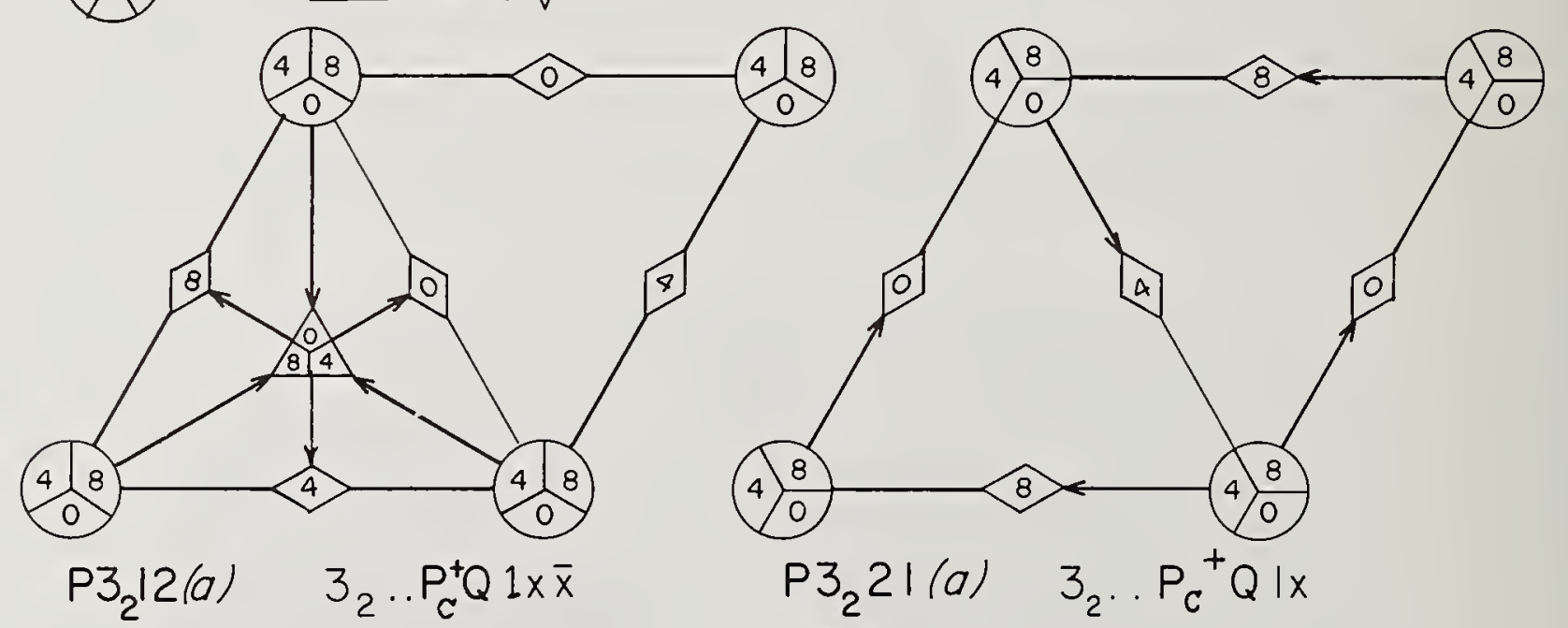

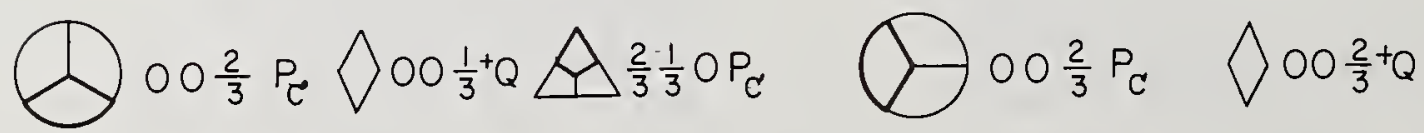

Fig. 56. Hexagonal univariant and bivariant Weissenberg complexes. Heights in twelfths of $c$. (See Section 7.32 , p. 19.) 


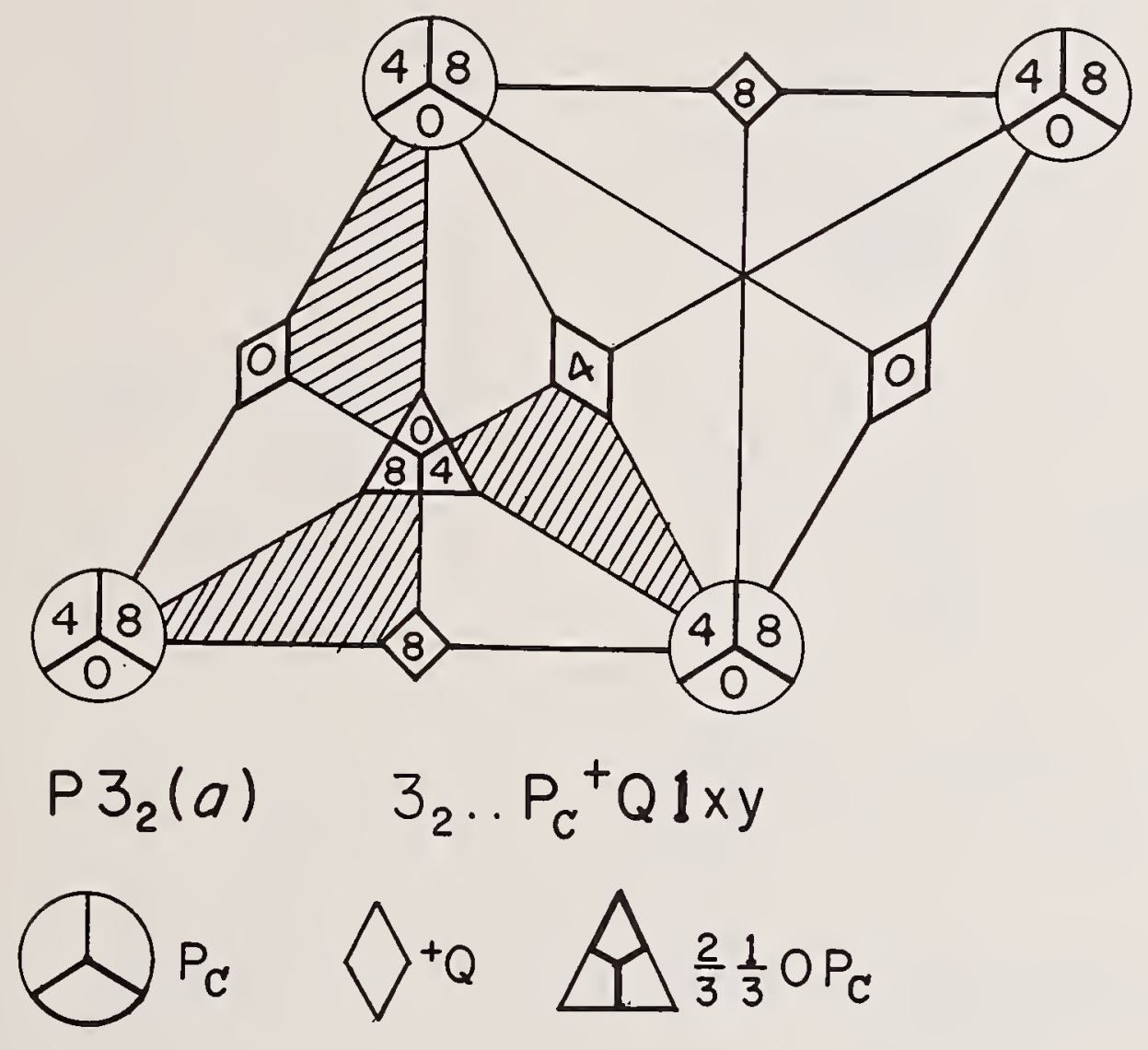

Fig. 57. One more hexagonal bivariant Weissenberg complex. Heights in twelfths of $c$. (See Section $7.32, p$. 19.) 

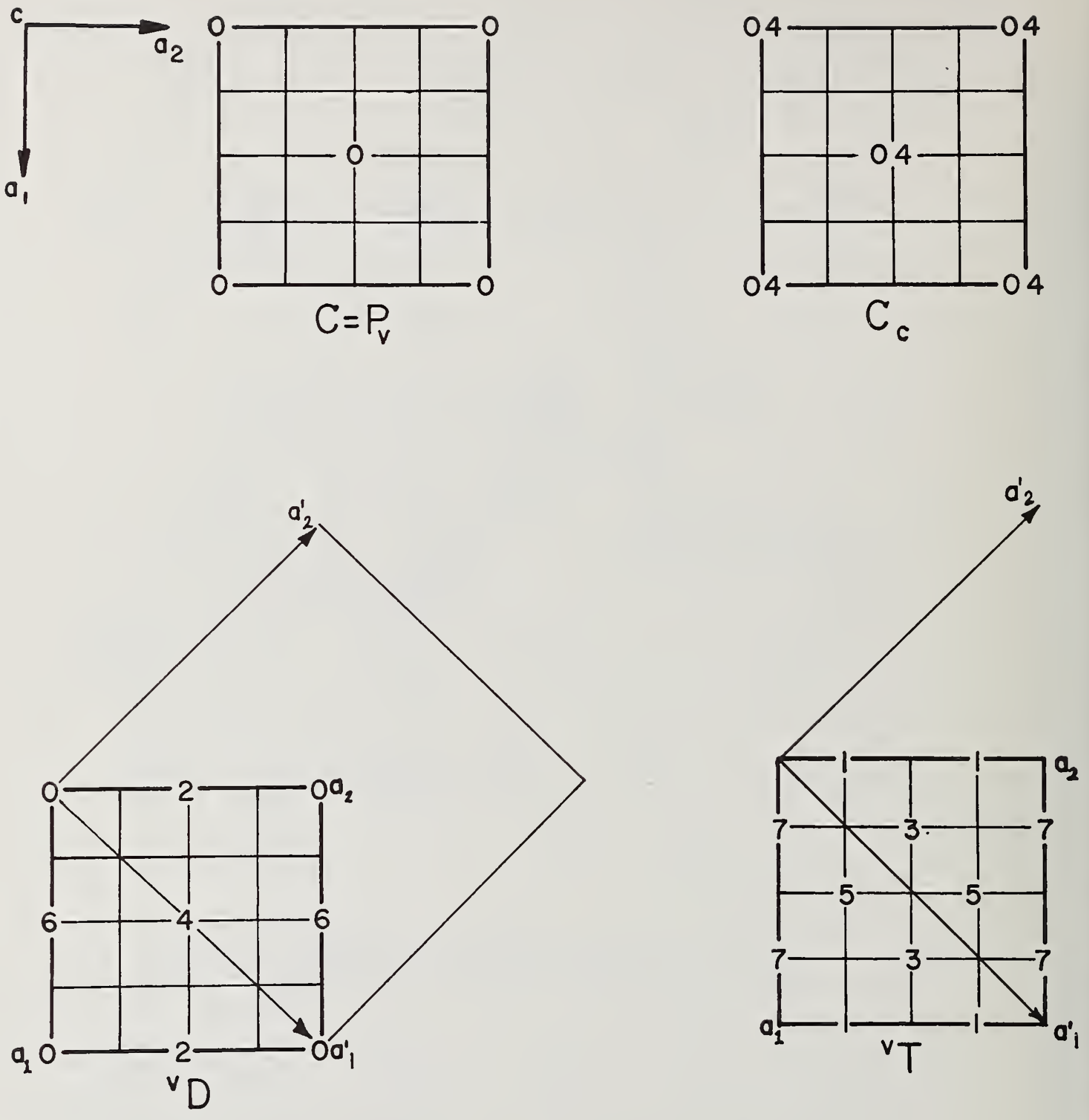

Fig. 58. Four representations of tetragonal invariant Weissenberg complexes that do not appear on the cubic drawings. Heights in eighths of $c$. 


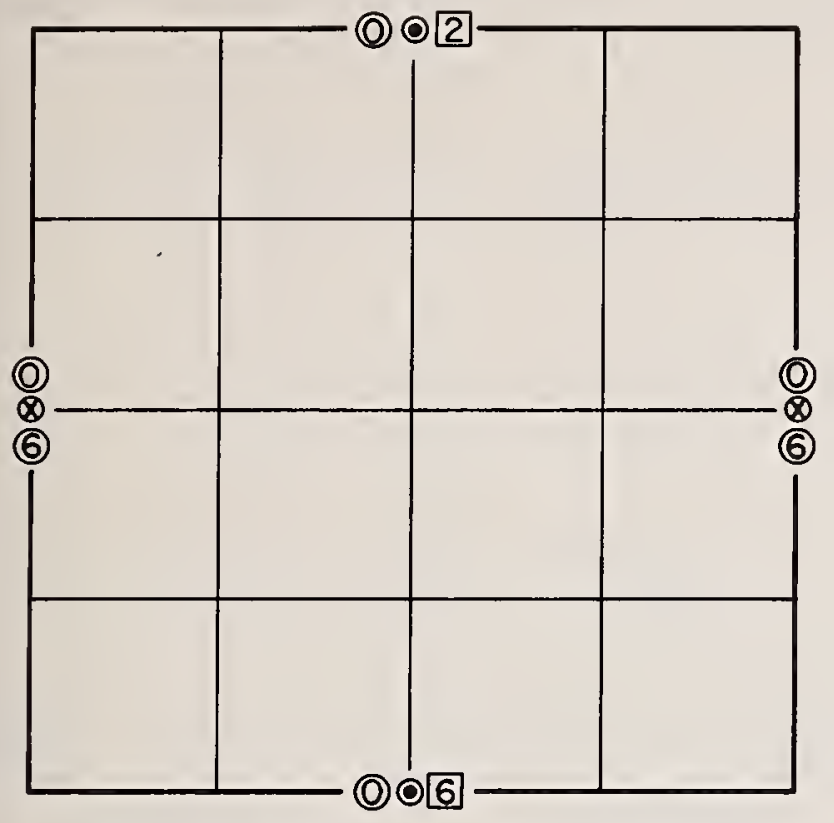

$\mathrm{P} 4 / \mathrm{nmm}(\mathrm{c}) \quad .2 \mathrm{CI} 1 \mathrm{z}$

$\bigcirc \circ \frac{1}{2} O C \quad \square O \frac{1}{2} \frac{1}{4} I$
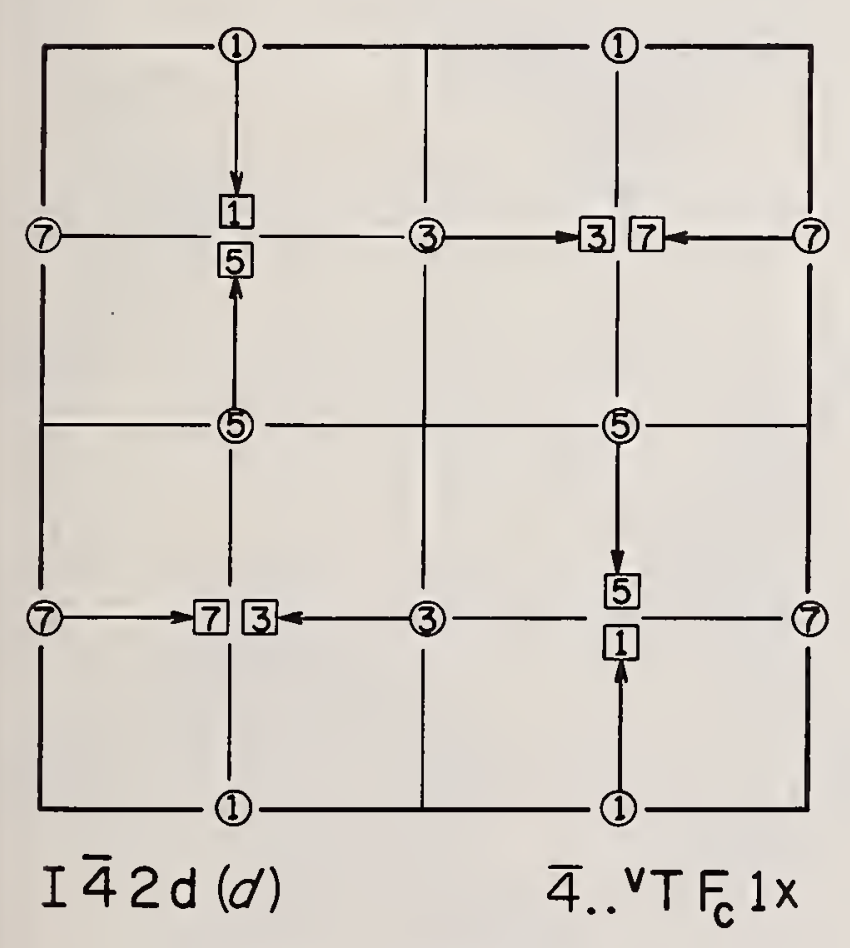

O VT $\square \frac{1}{4} \frac{1}{4} \frac{1}{8} \mathrm{~F}_{\mathrm{c}}$
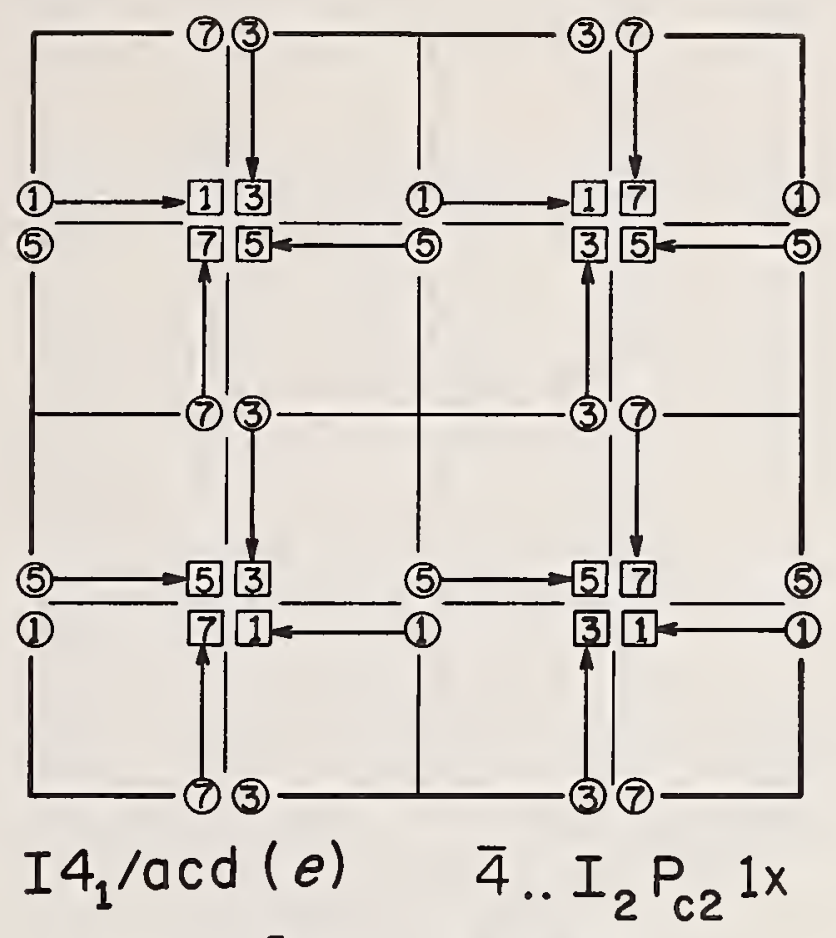

$\bigcirc \circ \frac{1}{4} \frac{3}{8} I_{2} \quad \square \frac{1}{4} \frac{1}{4} \frac{3}{8} P_{c 2}$

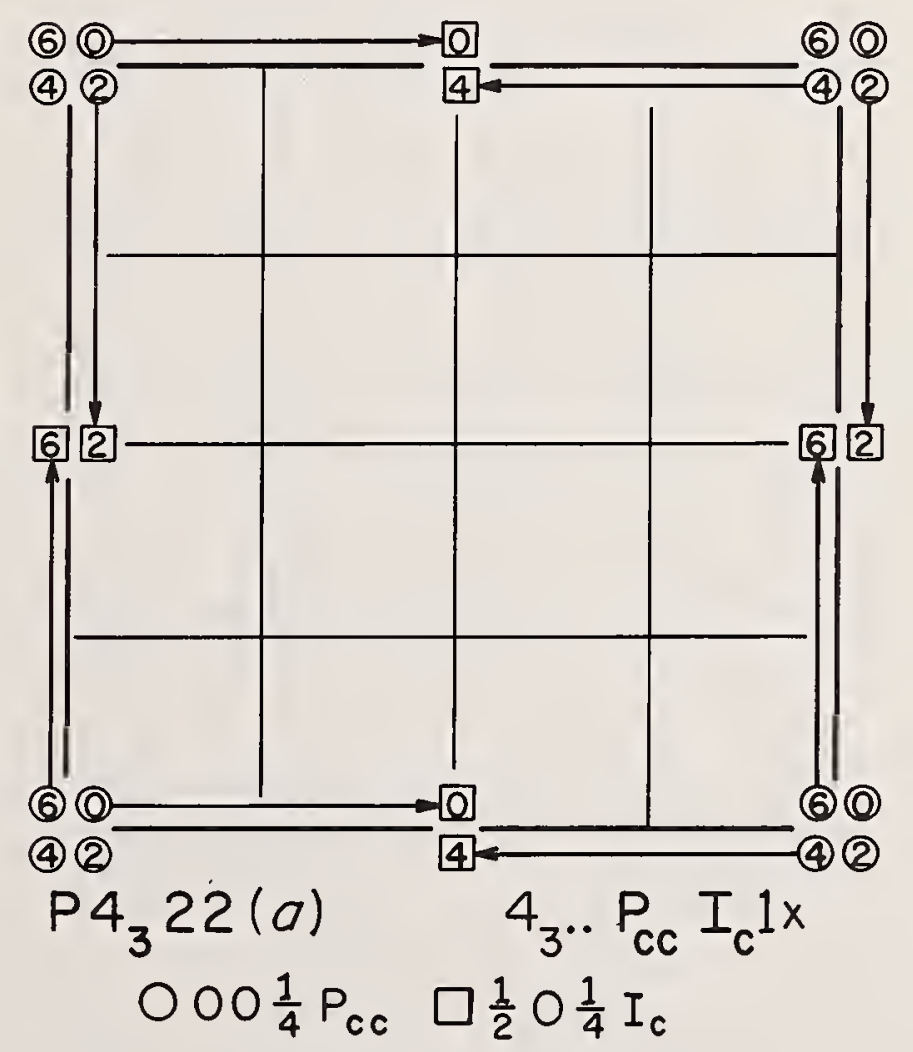

Fig. 59. Four tetragonal univariant Weissenberg complexes. Heights in eighths of $c$. (See Section 7.33.) 
(4)
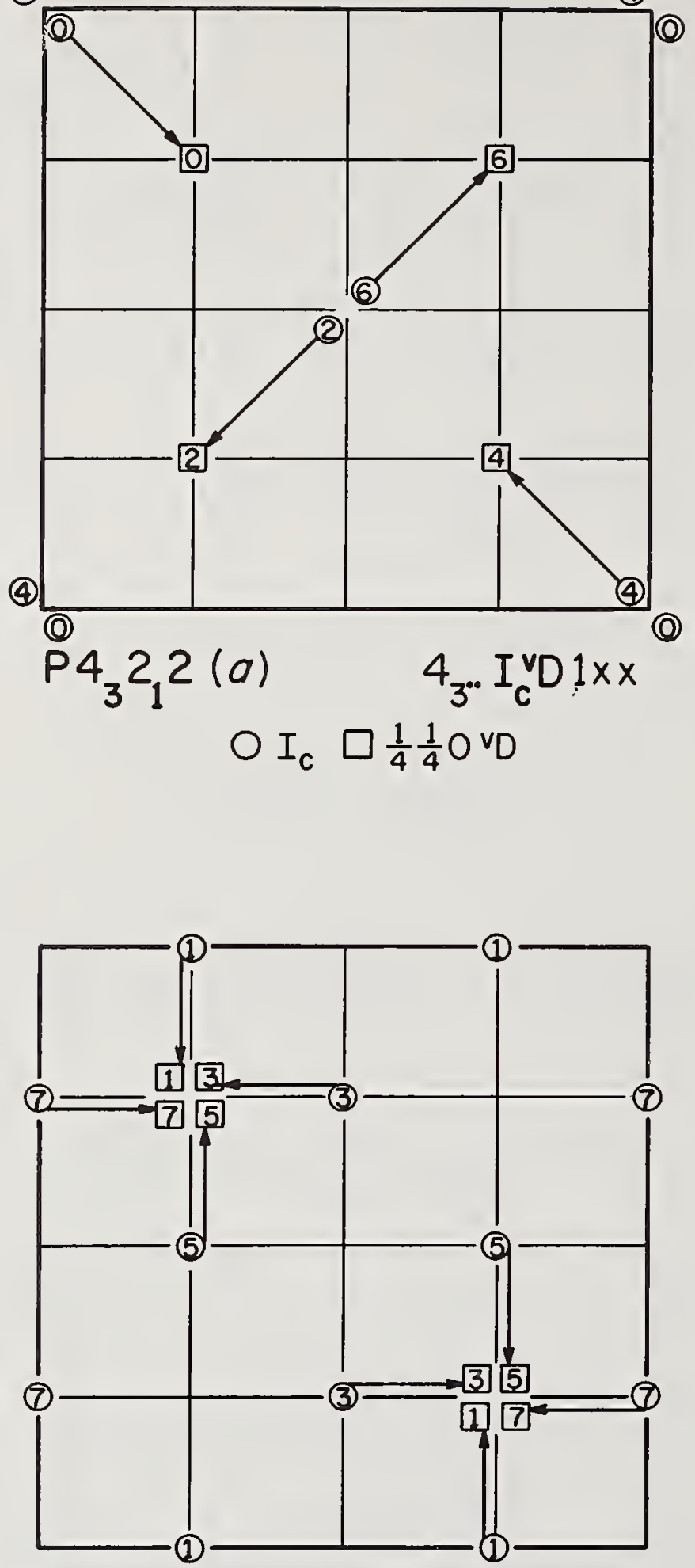

I 4 22 $(f)$

OVT $\square \frac{1}{4} \frac{1}{4} \frac{1}{8} C_{c c}$
(1) (3) (1) (3)

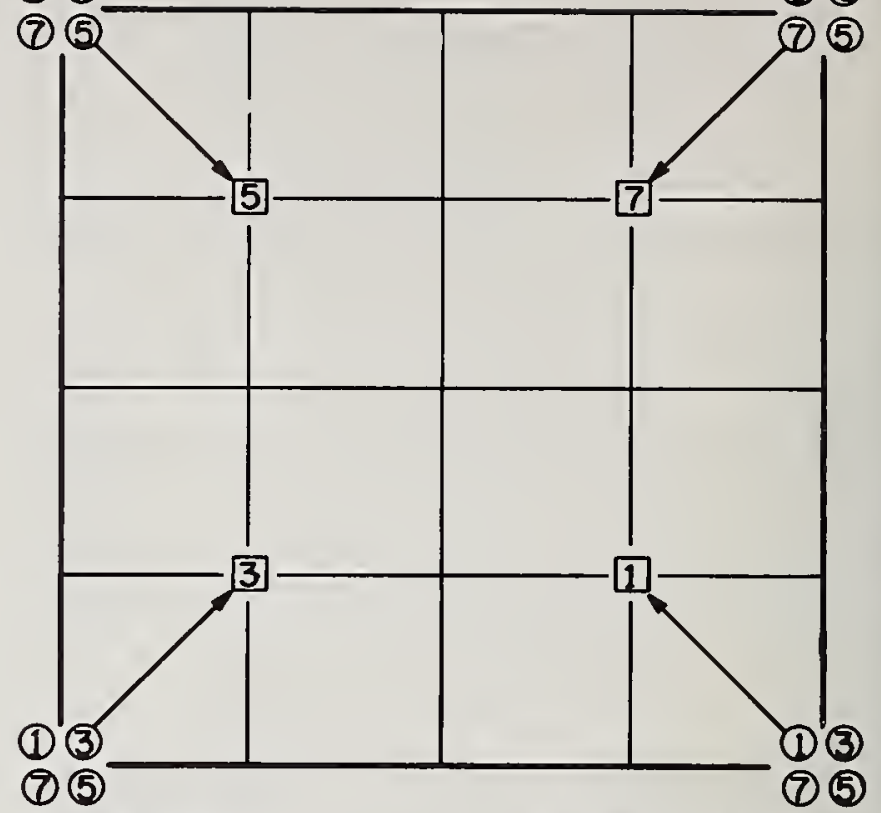

$\mathrm{P}_{3} 22(c)$

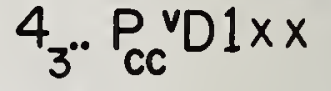

$\bigcirc 00 \frac{5}{8} P_{C C} \quad \square \frac{1}{4} \frac{1}{4} \frac{5}{8}{ }^{V} D$

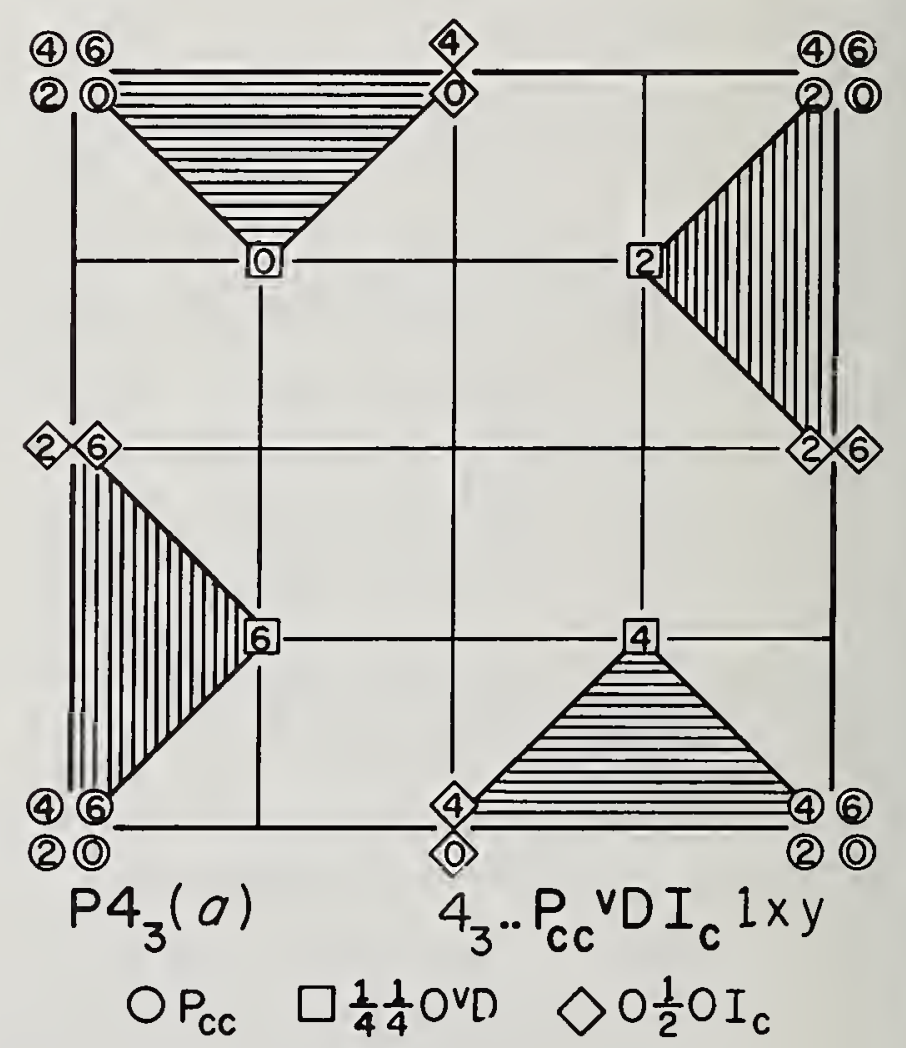

Fig. 60. Three more univariant tetragonal Weissenberg complexes and the only bivariant one. Heights in eighths of $c$. (See section 7.33.) 

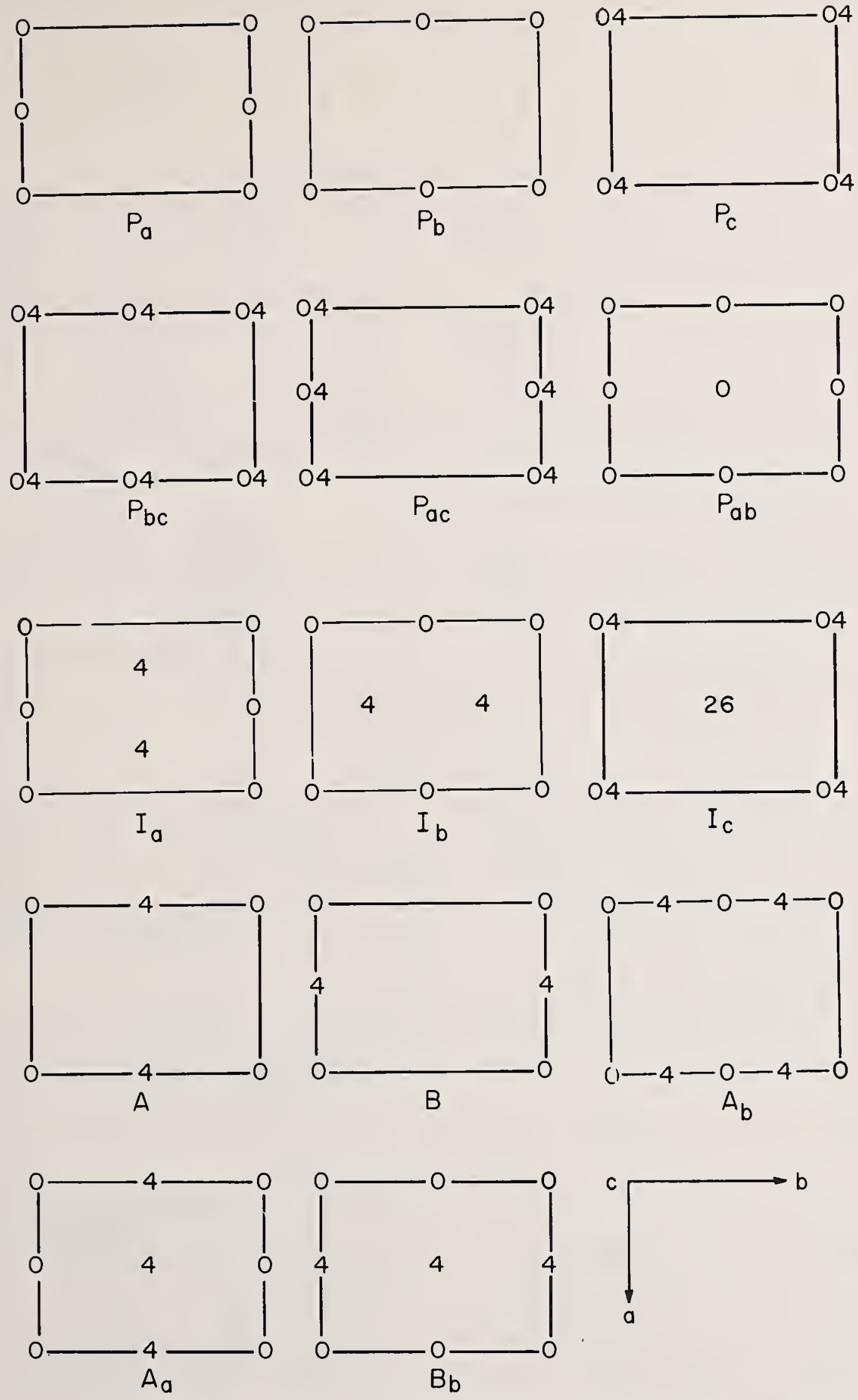

Fig. 61. Representations of orthorhombic invariant weissenberg complexes. Heights in eighths of $c$. For the invariant complexes themselves, look up cubic P, I, F, D, T (Fig.16) and tetragonal representation $\mathrm{C}$ (Fig.58), which are their topological analogs. 

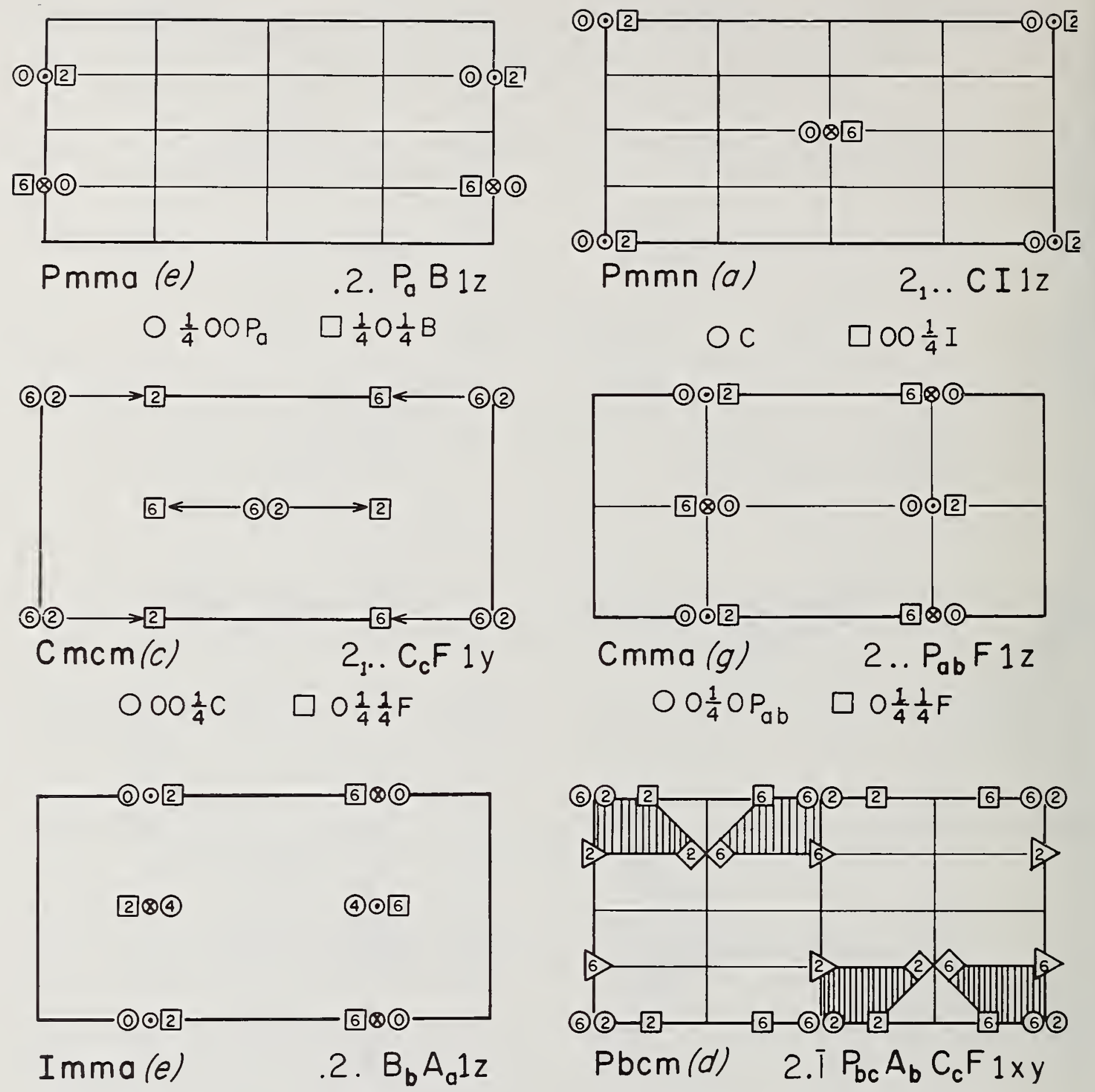
$\odot O \frac{1}{4} O B_{b}$
$\square \circ \frac{1}{4} \frac{1}{4} A_{a}$
Fig. 62. Five univariant and one bivariant orthorhombic Weissenberg complexes. Heights in eighths of $c$.

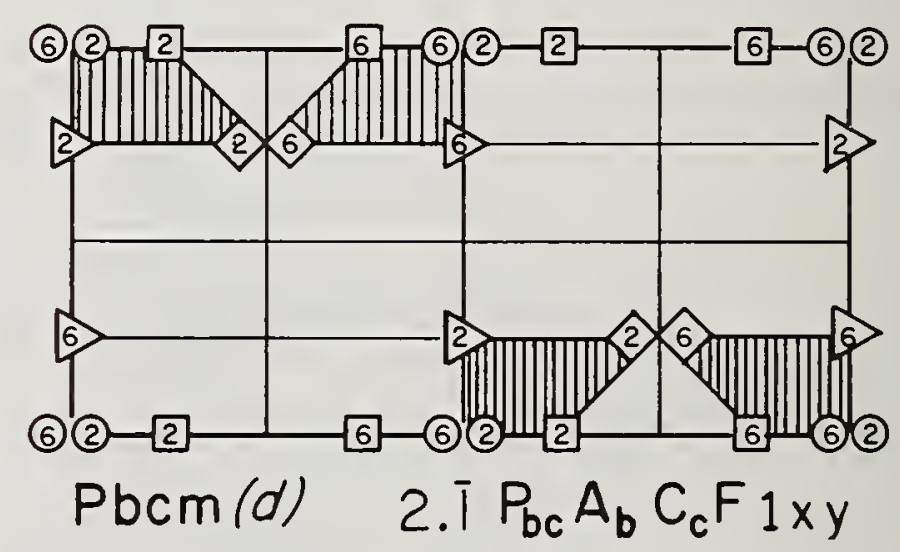



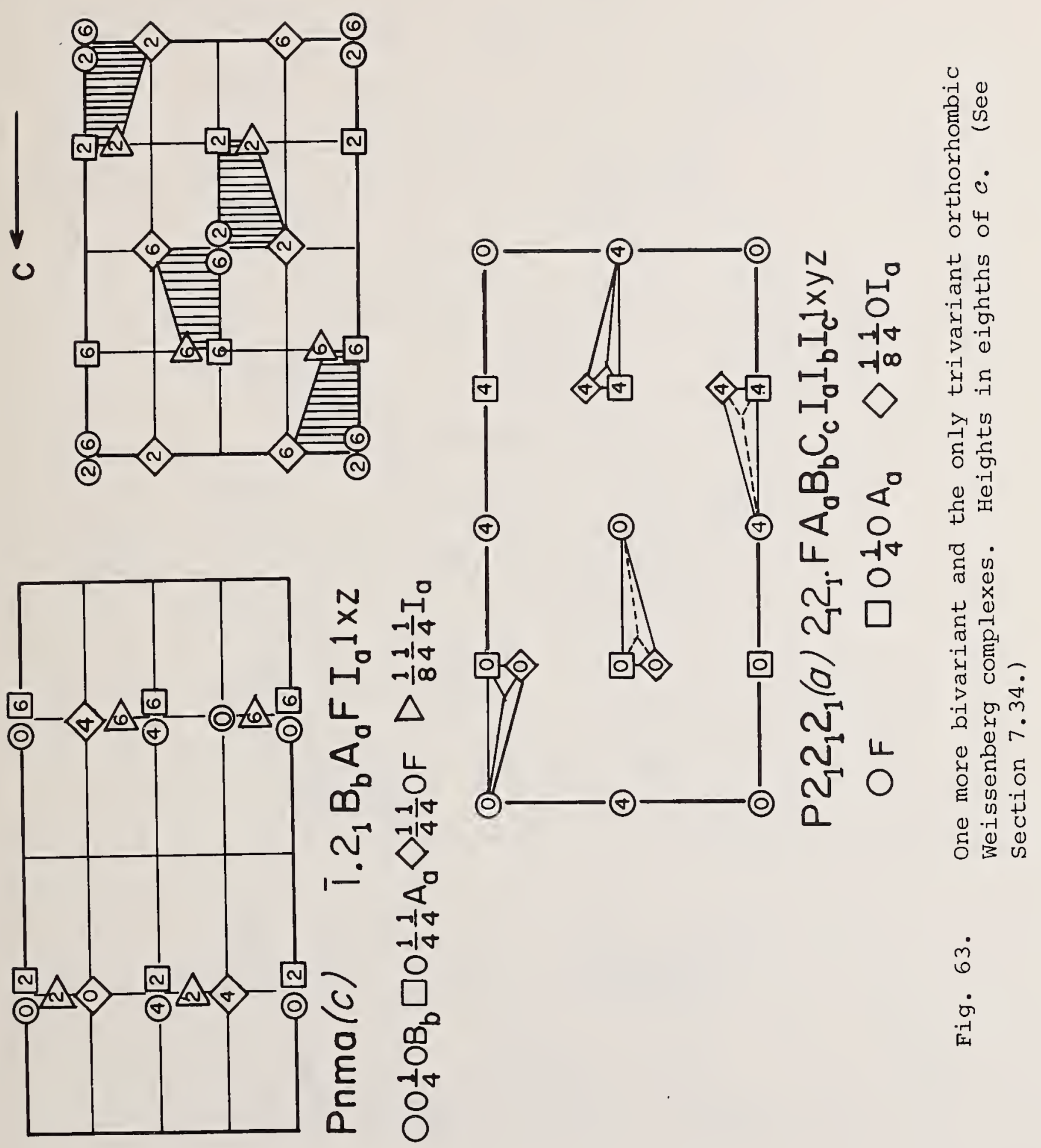

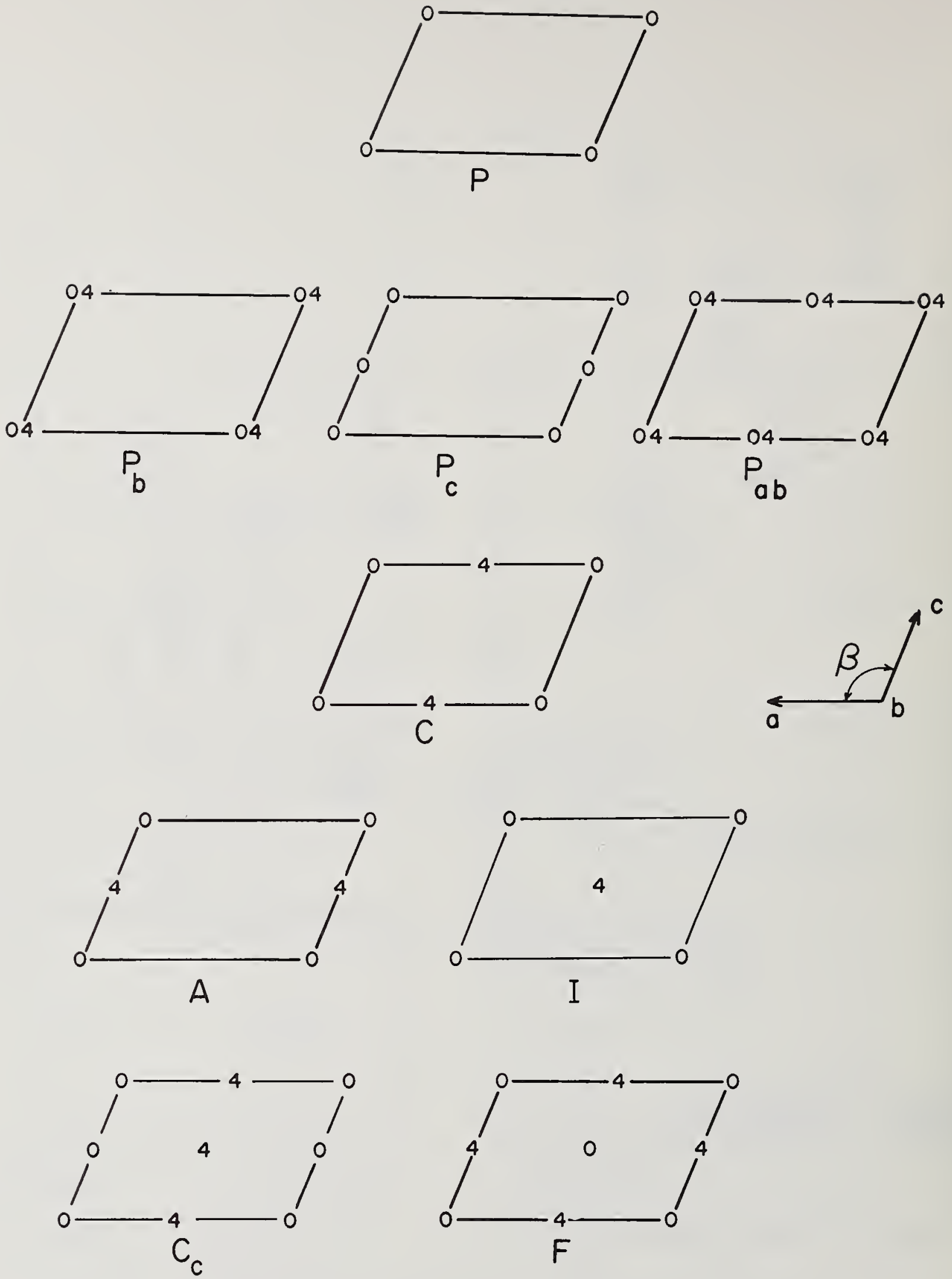

Fig. 64. The two monoclinic invariant Weissenberg complexes, in standard and alternate representations. Heights in eighths of $b$. 

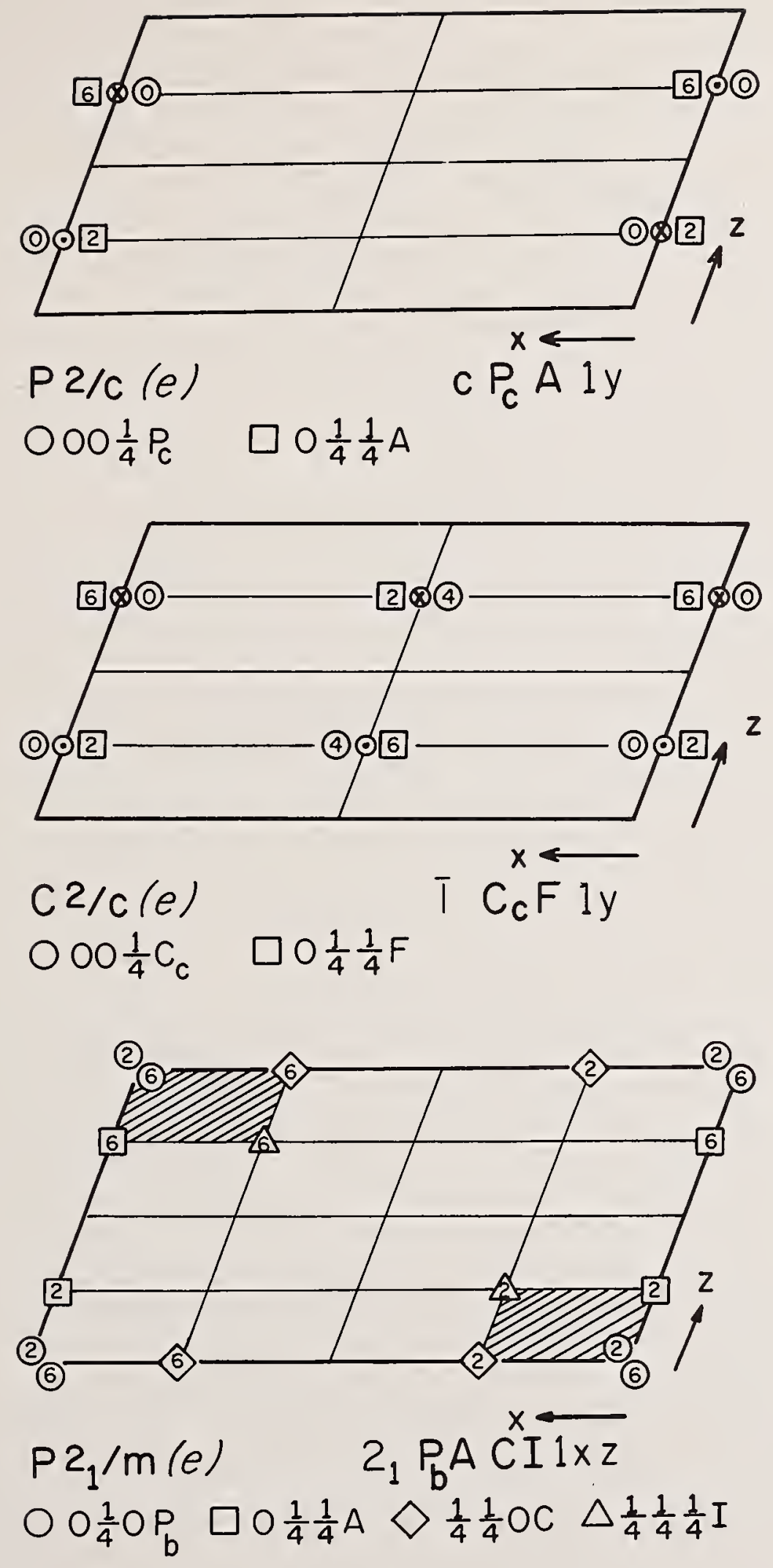

Fig. 65. The two univariant and the only bivariant monoclinic Weissenberg complexes. Heights in eighths of $b$. 

TABLES 
Table 1. Rules of Splitting

Point group:

nomopolar single. lst Site 2nd Site 3rd Site Point-complex primary polar split. Symm. Split. Symm. Split. Symm. symbol axis axis

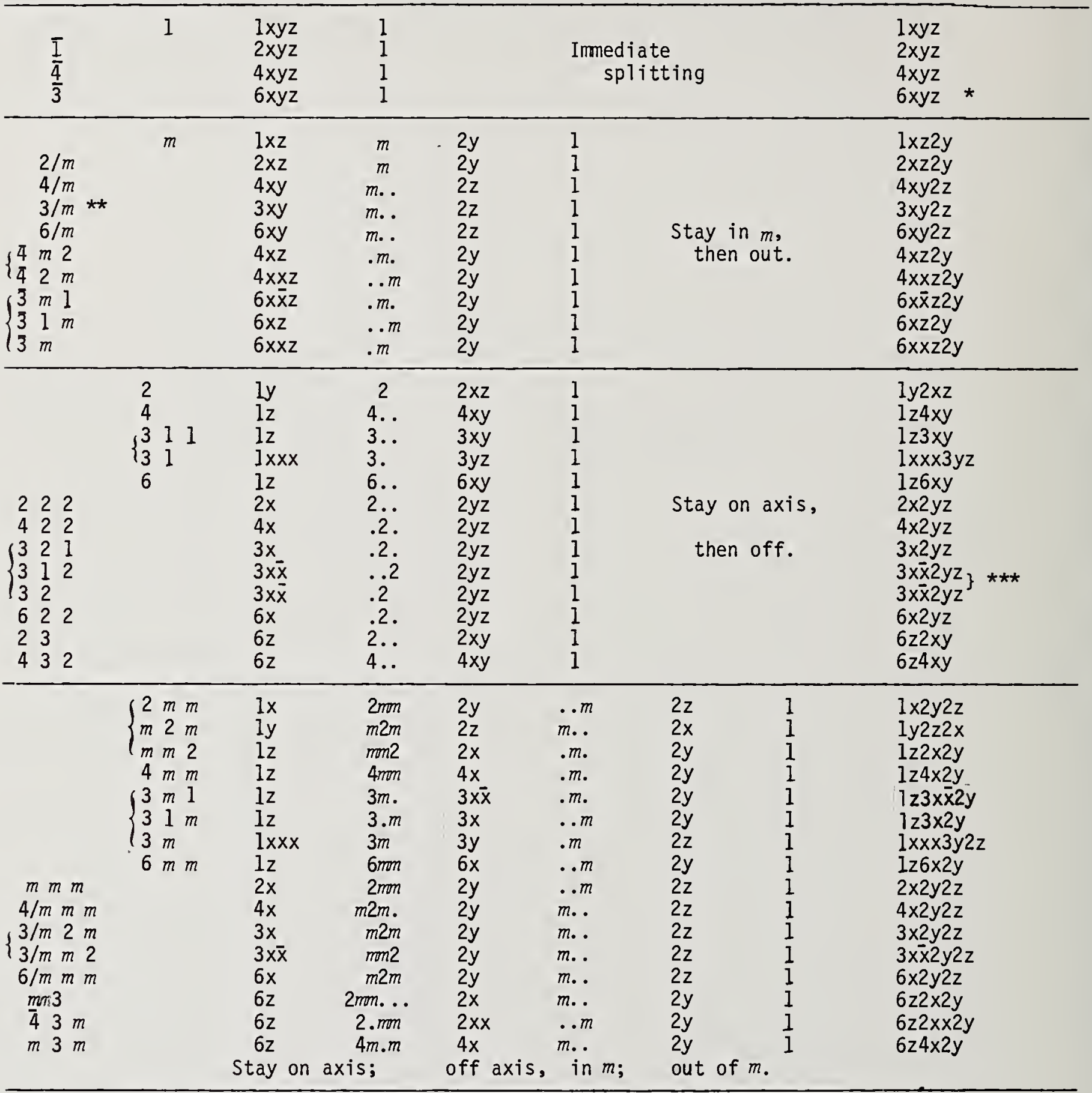

* The symbol is the same in hexagonal and rhombohedral axes.

$\star * \quad 3 / m=\overline{6}$

*** The same symbol serves for 312 in the hexagonal system and for 32 in rhombohedral co-ordinates. The preceding symbol is used both for 321 in the hexagonal system and for 32 in hexagonal co-ordinates. 
Co-ordination polyhedron. Dual crystal form. Site-set symbol. Co-ordinates. 0ccurrences.

Tetrahedron. Tetrahedron.

4xxx: $\quad x x x ; x \bar{x} \bar{x} \kappa \quad \overline{4} 3 m(a) ; \quad \overline{4} 3 m(b) ; \quad 23(a) ; \quad 23(b)$.

Octahedron. Cube.

6z: $\quad \pm(00 z) \kappa \quad m 3 m(a) ; \quad \overline{4} 3 m(c) ; \quad 432(a) ; \quad m 3(a) ; \quad 23(c)$.

Cube. Octahedron.

8xxx: $\quad \pm(x x x ; x \bar{x} \bar{x} k) \quad m 3 m(b) ; \quad 432(b) ; \quad m 3(b)$.

Cuboctahedron. Rhomb-dodecahedron.

12xx: $\quad \pm(x \times, 0) \quad m 3 m(c) ; \quad 432(c)$.

Dihexahedron + octahedron. Dihexahedron (or pentagon-dodecahedron).

6z2x: $\quad \pm(x, 0, \pm z) \kappa \quad m 3(c)$

Truncated tetrahedron. Trigon-tritetrahedron. (For $\quad \mid \begin{aligned} & x \\ & z|<| z \mid \\ & \text { Cube }+2 \text { tetrahedra. }\end{aligned}$.)
Tetragon-tritetrahedron.

6z2xx: $\quad(x x z ; \bar{x} \bar{x} z)_{k} ; x \bar{x} \bar{z} \pi \quad \overline{4} 3 m(d)$.

Pentagon-Tritetrahedron +2 tetrahedra. Pentagon-Tritetrahedron.

6z2xy: $\quad(x y z ; \bar{x} \bar{y} z ; \bar{x} y \bar{z} ; \bar{x} \bar{y} z) \kappa 23(d)$.

Truncated octahedron. Tetrahexahedron.

6z4x: $\quad \pm(x, 0, \pm z) \pi \quad m 3 m(d)$.

Truncated cube. Trigon-trioctahedron. (For $|z|<|x|+$ )

Rhombicuboctahedron. Tetragon-trioctahedron. (For $|x|<|z|$.)

6z4xx: $\quad \pm(x, x, \pm z k ; x \bar{x} z \pi) \quad m 3 m(e)$.

Snub cube. Pentagon-trioctahedron.

6z4xy: $\quad(x y z ; x \bar{y} \bar{z} ; \bar{x} y \bar{z} ; \bar{x} \bar{y} z ; \bar{y} \bar{x} \bar{z} ; \bar{y} x z ; y \bar{x} z ; y x \bar{z})_{\kappa} \quad 432(d)$.

Cube + octahedron + dihexahedron. Didodecahedron.

6z2x2y: $\quad \pm(x, y, \pm z ; x, \bar{y}, \pm z) \kappa m 3(d)$.

Cube +2 tetrahedra. Hexatetrahedron.

6z2xx2y: $\quad(x y z ; x \bar{y} \bar{z} ; \bar{x} y \bar{z} ; \bar{x} \bar{y} z) \pi \overline{4} 3 m(e)$.

Truricated cuboctahedron. Hexoctahedron.

6z4x2y: $\quad \pm(x, y, \pm z ; x, \bar{y}, \pm z) \pi m 3 m(f)$.

1 Limiting cases not included. 
Table 3. Site Sets ${ }^{1}$ Found in the Hexagonal and Rhombohedral Systems (in hexagonal co-ordinates)

Co-ordination polyhedron. Dual crystal form. Site-set symbol. Co-ordinates. Occurrences.

Point. Monohedron.

1z: $\quad 00 z$

$\begin{array}{llll}6 \mathrm{~mm}(\mathrm{a}) ; & 6 \mathrm{~mm}(\mathrm{~b}) ; & 6(\mathrm{a}) ; & 6(\mathrm{~b}) ; \\ 3 \mathrm{ml}(\mathrm{a}) ; & 3 \mathrm{ml}(\mathrm{b}) ; & & \\ 31 \mathrm{~m}(\mathrm{a}) ; & 31 \mathrm{~m}(\mathrm{~b}) ; & 311(\mathrm{a}) ; & 311(\mathrm{~b})\end{array}$

Line segment (collinear with origin). Parallelehedron.
$2 z:$
$0,0, \pm z$
$6 / \mathrm{mmm}(\mathrm{a}) ; \quad \overline{6} \mathrm{~m} 2(\mathrm{a}) ;$
$6 / \mathrm{m}(\mathrm{a}) ; \quad \overline{6}(\mathrm{a})$
$321(a) \quad 312(a)$
$\overline{6} 2 m(a) ; \quad 622(a) ;$
$3 \mathrm{ml}(\mathrm{a}) ; \quad \overline{3} 1 \mathrm{~m}(\mathrm{a})$;
$311(a)$.

Trigon coplanar with origin. Trigonal prism.

$\begin{array}{lllll}3 x \bar{x}: & x \bar{x} 0 & \overline{6} m 2(b) ; & \overline{6} m 2(c) ; & \\ 3 x: & x 00^{2} & \overline{6} 2 m(b) ; & 321(c) ; & \overline{6} 2 m(c) ; 321(b) .\end{array}$

Trigon. Trigonal pyramid.

$\begin{array}{lll}1 z 3 x \bar{x}: & x \bar{x} z & 3 m 1(c) . \\ 1 z 3 x & x 0 z^{\imath} & 31 m(c) . \\ 1 z 3 x y: & x y z z_{1} & 311(c) .\end{array}$

Truncated trigon coplanar with origin. Ditrigonal prism.

$3 x \bar{x} 2 y:$

$3 x 2 y:$

$$
\begin{array}{ll}
(x y 0, \bar{y} \bar{x} 0)_{1} & \overline{6 m} 2(e) . \\
\left(x y{ }_{\tau}\right)_{1} & 62 m(e) .
\end{array}
$$

Truncated trigon. Ditrigonal pyramid.

$\begin{array}{lll}1 z 3 x \bar{x} 2 y: & (x y z, \bar{y} \bar{x} z)_{1} & 3 m l(d) . \\ 1 z 3 x 2 y: & \left(x y O_{\tau}\right)_{l} & 31 \mathrm{~m}(d) .\end{array}$

Hexagon coplanar with origin. Hexagonal prism.
$6 x:$
$( \pm x, 0,0)$
$6 x \bar{x}:$
$\pm\left(x \times 0^{2}\right)^{2}$
$\pm\left(x y 0^{2}\right)$
$6 / \mathrm{mmm}(\mathrm{b}) ; \quad 622(\mathrm{~b}) ; \quad 3 \mathrm{ml}(\mathrm{b})$.
$6 x y:$
$6 / \mathrm{mmm}(\mathrm{c}) ; 622(\mathrm{c}) ; \quad \overline{3} 1 \mathrm{~m}(\mathrm{~b})$.

Hexagon. Hexagonal pyramid.

$\begin{array}{lll}1 z 6 x \bar{x}: & \left(x \bar{x} z_{\tau}\right)_{1} & 6 m(c) . \\ 1 z 6 x: & ( \pm x, 0, z)_{2} & 6 m(d) . \\ 1 z 6 x y: & (x y z, x y z)_{2} & 6(c) .\end{array}$

Trigonal prism + pinacoid. Trigonal dipyramid.
$3 \times \bar{x} 2 z:$
$3 \times 2 z:$
$3 x y 2 z:$

$$
\begin{aligned}
& (x, \bar{x}, \pm z) \tau \\
& (x, 0, \pm z)^{\tau} \\
& (x, y, \pm z)_{\tau}^{\tau}
\end{aligned}
$$
$\overline{6} \mathrm{~m} 2(\mathrm{~d})$
$\overline{6} 2 \mathrm{~m}(\mathrm{~d})$.
$\bar{\sigma}(\mathrm{c})$.

1 Limiting cases not included. 
Table 3 (concluded).

Trigonal trapezohedron + pinacoid. Trigonal trapezohedron.
$3 \times 2 y z:$
$3 x \bar{x} 2 y z:$
$(x y z ; y x \bar{z}) \tau$
321 (d).
$312(d)$.

Trigonal antiprism. Rhombohedron.
ōxēz:
$6 x z:$

$$
\begin{aligned}
& \pm(x \bar{x} z) \\
& \pm(x 0 z) \tau \\
& \pm(x y z) \tau \\
& \tau
\end{aligned}
$$
$3 \mathrm{ml}(\mathrm{c})$.
$6 x y z:$$$
\overline{3} 7 \mathrm{~m}(\mathrm{c}) \text {. }
$$$$
\text { उิ11(b). }
$$

Truncated hexagon coplanar with origin. Dihexagonal prism.
$6 x 2 y:$
$\pm\left(\mathrm{xyO}_{\tau}\right)_{\mathrm{l}}$
$6 / \mathrm{mmm}(f)$.

Truncated hexagon. Dihexagonal pyramid.

$1 z 6 \times 2 y:$

$$
\left((x y z ; \bar{x} \bar{y} z)_{\tau}\right)_{l} \quad 6 m m(e) .
$$

Pinacoid + rhombohedron. Hexagonal scalenohedron.

$\begin{array}{lll}6 x \bar{x} z 2 y: & \pm(x y z ; \bar{y} \bar{x} z)_{\tau} & \overline{3} m 7(d) . \\ 6 x z 2 y: & \pm\left(x y z{ }_{\tau}{ }_{l}\right. & \overline{3} 7 m(d) .\end{array}$

Truncated trigonal prism + pinacoid. Ditrigonal dipyramid.
$3 x \bar{x} 2 y 2 z:$
$3 x 2 y 2 z$ :
$(x, y, \pm z ; \bar{y}, \bar{x}, \pm z)_{\tau}$
$\left(x, z, \pm z_{\tau}\right)_{\tau}$
$\overline{6} \mathrm{~m} 2(f)$.
$\overline{6} 2 \mathrm{~m}(f)$.

Hexagonal prism + pinacoid. Hexagonal dipyramid.
$6 \times 2 z:$
$6 x \bar{x} 2 z:$
$6 x y 2 z:$

$$
\begin{aligned}
& ( \pm x, 0, \pm z)^{\tau} \\
& (x, x, \pm z \tau)^{\tau} \\
& \pm(x, y, \pm z)_{2}
\end{aligned}
$$
$6 / \mathrm{mmm}$ (d).
$6 / \mathrm{mmm}$ (e).
$6 / m(c)$.

Hexagonal trapezohedron + pinacoid. Hexagonal trapezohedron.

6x2yz: $\quad(x y z, \bar{x} \bar{y} z, y x \bar{z}, \bar{y} \bar{x} \bar{z})_{\tau} \quad$ 622(d).

Truncated hexagonal prism + pinacoid. Dihexagonal dipyramid.

6x2y2z: $\quad \pm\left(x, y, \pm z_{\tau}\right)_{2} \quad 6 / \mathrm{mmm}(\mathrm{g})$. 
Table 4. Site Sets ${ }^{1}$ Found in the Rhombohedral System (in rhombohedral co-ordinates)

Co-ordination polyhedron. Dual crystal form. Site-set symbol. Co-ordinates. Occurrences. Point. Monohedron (pedion).
Ixxx:
$x x x$
$3 m(a) ; \quad 3 m(b) ; \quad 31(a) ; \quad 31(b)$.

Line segment (collinear with origin). Parallelehedron (pinacoid).

2xxx: $\pm(x x x) \quad \overline{3} m(a) ; 32(a) ; \quad \overline{3} l(a)$.

Trigon coplanar with origin. Trigonal prism.

$3 x \bar{x}: \quad x \bar{x} 0 \kappa \quad 32(b) ; 32(c)$.

Trigon. Trigonal pyramid.

$\begin{array}{lll}\text { Ixxx3z: } & x x z \kappa & 3 m(c) . \\ 1 \times x \times 3 y z: & x y z \kappa & 3 l(c) .\end{array}$

Hexagon coplanar with origin. Hexagonal prism.

$6 x \bar{x}: \quad \pm(x \bar{x} 0 k) \quad 3 m(b)$.

Truncated trigon. Ditrigonal pyramid.

1xxx3y2z: $\quad x y z \pi \quad 3 m(d)$.

Trigonal trapezohedron + pinacoid. Trigonal trapezohedron.

$\begin{array}{lll}3 x \bar{x} 2 y z: & (x y z, \bar{x} \bar{z} \bar{y})_{\kappa} & 32(d) .\end{array}$

Trigonal antiprism. Rhombohedron.
6xxz:
6xyz:

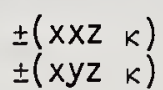
$3 m(c)$.
31(b).

Pinacoid + rhombohedron. Hexagonal scalenohedron.

6xxz2y: $\quad \pm(x y z \pi) \quad 3 m(d)$.

1 Limiting cases not included. 
Co-ordination polyhedron. Dual crystal form. Site-set symbol. Co-ordinates. Occurrences

Point. Monohedron (pedion).

1z: $-00 z \quad 4 m m(a) ; 4 m m(b) ; 4(a) ; \quad 4(b)$.

Line segment (collinear with origin). Parallelehedron (pinacoid).

2z: $\quad \pm(00 z) \quad 4 / m m m(a) ; \quad \overline{4} 2 m(a) ; \quad \overline{4} 2(a) ; \quad 422(a) ; 4 / m(a) ; \overline{4}(a)$.

Tetragon (square) coplanar with origin. Tetragonal prism.

$\begin{array}{lllll}4 x: & \pm(x 00) \tau & 4 / m m m(b) ; & \overline{4} 2 m(b) ; & 422(b) . \\ 4 x x: & \pm(x, \pm x, 0) & 4 / m m(c) ; & 4 m 2(b) ; & 422(c) . \\ 4 x y: & \pm(x y 0, y x 0) & 4 / m(b) . & & \end{array}$

Tetragon. Tetragonal pyramid.

1z4x: $\quad \pm x, 0, z ; 0, \pm x, z . \quad 4 m m(c)$.

1 z4xx: $\quad \pm x, \pm x, z$. $\quad 4 \mathrm{~mm}(\mathrm{~d})$.

lz4xy: $\quad(+++,-+)(x y z, y \bar{x} z) . \quad 4(c)$.

Tetragonal tetrahedron. Tetragonal tetrahedron.

$\begin{array}{lll}4 x z: & \pm x, 0, z ; 0, \pm x, \bar{z} . & \overline{4} m 2(c) . \\ 4 x x z: & x x z ; x \bar{x} z ; x \bar{z} \tau, \overline{4} 2 m(c) . \\ 4 x y z: & x y z, x y z, y x z, \bar{y} x \bar{z} & \overline{4}(b) .\end{array}$

Truncated tetragon coplanar with origin. Ditetragonal prism.

$4 \times 2 y: \quad \pm(x y 0 ; \bar{y} \times 0)_{\tau} \quad 4 / \mathrm{mmm}(f)$.

Tetragonal tetrahedron + pinacoid. Tetragonal scalenohedron.

4xz2y: $\quad \pm x, \pm y, z ; \underline{z} \pm y, \pm \underline{x}, \bar{z} \dot{\bar{z}}-\quad \overline{4} m 2(\mathrm{~d})$.

4xxz2y: (xyz; $\bar{x} \bar{y} ; y \bar{x} \bar{z} ; \bar{y} x \bar{z})_{\tau} \quad \overline{4} 2 \mathrm{~m}(\mathrm{~d})$.

Truncated tetragon. Ditetragonal pyramid.

1z4x2y: $\quad \pm x, \pm y, z ; \pm y, \pm x, z . \quad 4 m m(e)$.

Tetragonal prism + pinacoid. Tetragonal dipyramid.
$4 x 2 z: \quad \pm(x, 0, \pm z)_{\tau}$
4xx2z: $\quad \pm(x, x, \pm z ; \underline{x}, \bar{x}, \pm z)$
$4 x y 2 z: \quad \pm(x, y, \pm z ; \bar{y}, x, \pm z)$
$4 / \mathrm{mmm}(\mathrm{d})$.
$4 / \mathrm{mmm}(\mathrm{e})$.
$4 / m(c)$.

Tetragonal trapezohedron + pinacoid. Tetragonal trapezohedron.

4x2yz: $\quad x y z ; \bar{x} \bar{y} z ; \bar{x} y \bar{z} ; x \bar{y} \bar{z} ; \bar{y} \bar{x} \bar{z} ; y x \bar{z} ; y \bar{x} z ; \bar{y} x z \quad 422(d)$.

Truncated tetragonal prism + pinacoid. Ditetragonal dipyramid.

4x2y2z: $\quad(x, y, z ; \bar{y}, x, \pm z)_{\tau} \quad 4 / \mathrm{mmm}(g)$.

1 Limiting cases not included. 
Table 6. Site Sets 1 Found in the Orthorhombic System

Co-ordination polyhedron. Dual crystal form. Site-set symiol. Co-ordinates. Occurrences.

Point. Monohedron (pedion).

1z: $\quad 00 z . \quad m m 2(a), \quad m m 2(b)$

Line segment (collinear with origin). Parallelehedron (pinacoid).

\begin{tabular}{|c|c|c|}
\hline $2 x:$ & $\pm \times 00$ & $\mathrm{mmm}(\mathrm{a})$ \\
\hline $2 y:$ & $\pm 0 y 0$. & $\mathrm{mmm}(\mathrm{b})$ \\
\hline $2 z:$ & $\pm 00 \mathrm{z}$. & $\mathrm{mmm}(\mathrm{c})$ \\
\hline
\end{tabular}

Line segment. Dihedron.
1z2y:
$0, \pm y, z$
$\mathrm{mm} 2(\mathrm{c})$.
$1 z 2 x$ :
$\pm x, 0, z$
$\mathrm{mm} 2(\mathrm{~d})$.

Rectangle coplanar with origin. Rhombic prism.

$\begin{array}{lll}2 y 2 z: & \pm(0, y, \pm z) & m m(d) . \\ 2 x 2 z: & \pm( \pm x, 0, z) & m m m(e) . \\ 2 x 2 y: & \pm(x, \pm y, 0) & m m m(f) .\end{array}$

Rectangle. Rhombic pyramid.

1z2x2y: $\quad \pm x, y, z ; \pm x, \bar{y}, z \quad m m 2(e)$

Rhomb ic tetrahedron. Rhombic tetrahedron.

$\begin{array}{lll}2 x 2 y z: & x y z ; x \bar{y} \bar{z} ; \bar{x} y \bar{z} ; \bar{x} \bar{y} z & 222(d) .\end{array}$

Rectangular parallelepiped. Rhombic dipyramid.

2x2y2z: $\quad \pm(x, y, \pm z ; x, \bar{y}, \pm z) \quad m m(g)$.

1 Limiting cases not included. 
Co-ordination polyhedron. Dual crystal form. Site-set symbol. Co-ordinates. Occurrences. Point. Monohedron (pedion).

$\begin{array}{lll}\text { ly: } & \text { yyo } & 2(a), 2(b) . \\ \text { lxz: } & \text { xoz } & \text { m(a). }\end{array}$

Line segment (collinear with origin). Parallelehedron (pinacoid).

$\begin{array}{lll}2 y: & \pm(0 \mathrm{y} 0) & 2 / \mathrm{m}(\mathrm{a}) . \\ 2 \mathrm{xz}: & \pm(\mathrm{xOz}) & 2 / \mathrm{m}(\mathrm{b}) .\end{array}$

Line segment. Dihedron.
1y2xz:
1xz2y:
$x y z, \bar{x} y \bar{z}$
$2(c)$.

Rectangle coplanar with origin. Rhombic prism.

2xz2y: $\quad \pm(x, \pm y, z) \quad 2 / m(c)$.

Table 8. Site Sets ${ }^{1}$ Found in the Anorthic (Triclinic) System

Co-ordination polyhedron. Dual crystal form. Site-set symbol. Co-ordinates. Occurrences.

Point. Monohedron (pedion).

1xyz: $\quad x y z \quad 1(a)$.

Line segment (collinear with origin). Parallelehedron (pinacoid).

2xyz: $\quad \pm(x y z) \quad i(a)$.

1 Limiting cases not included. 


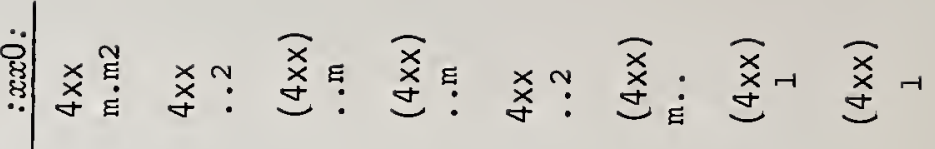

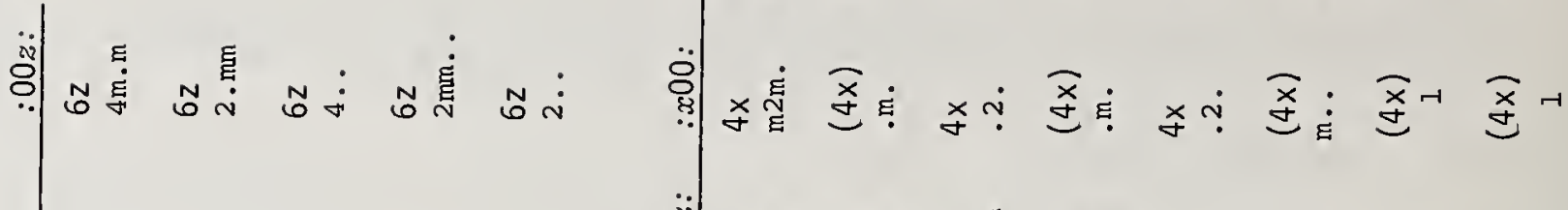

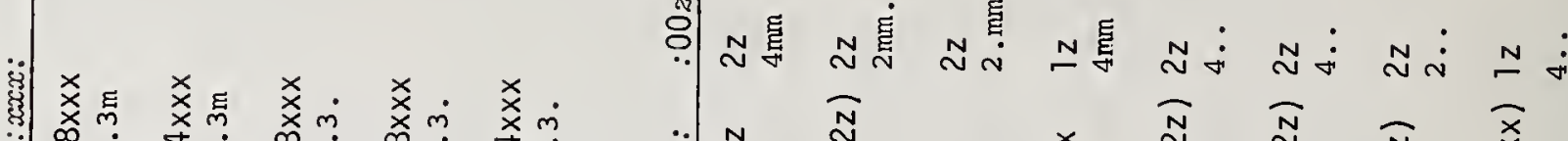

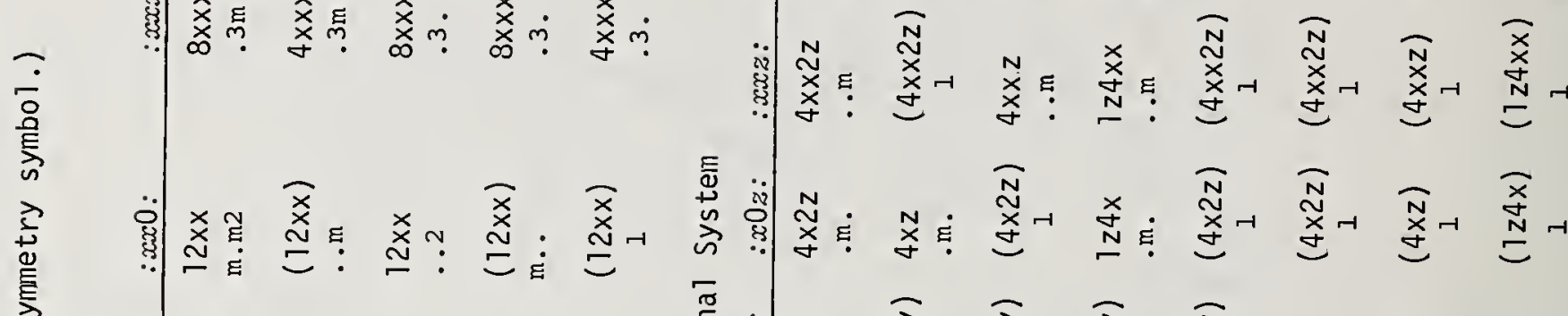

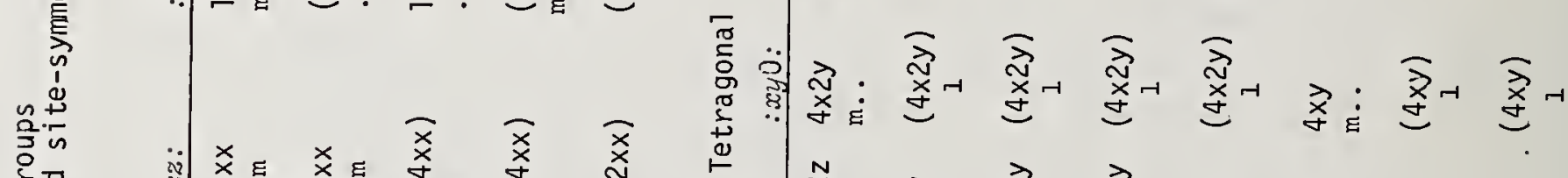

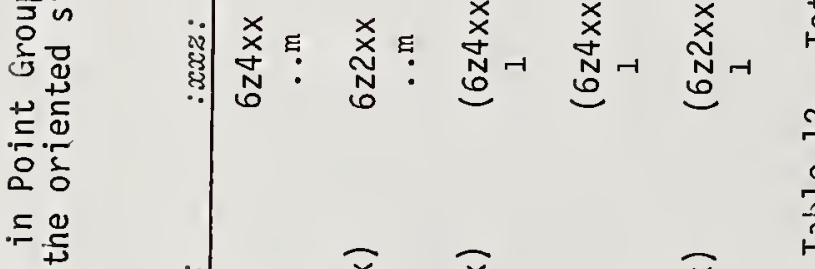

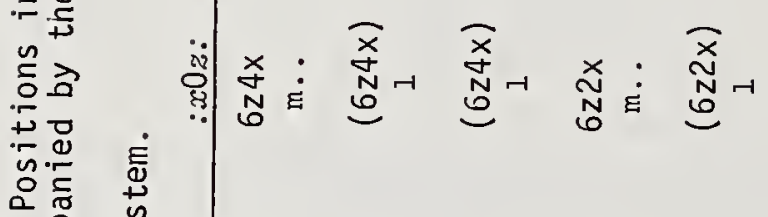

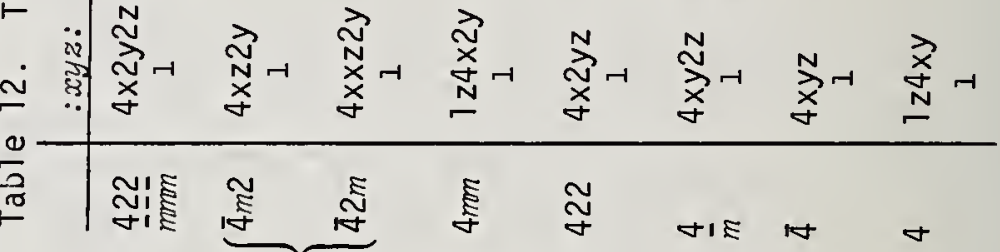

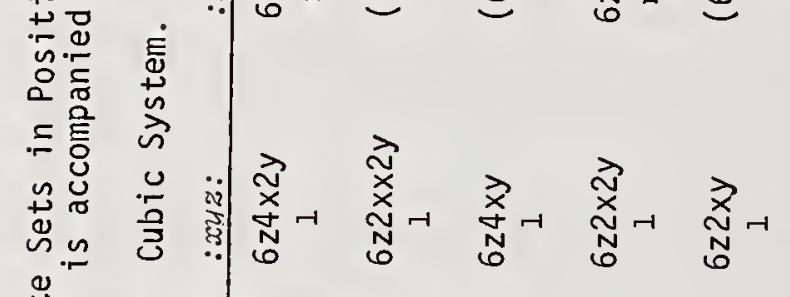
总兽

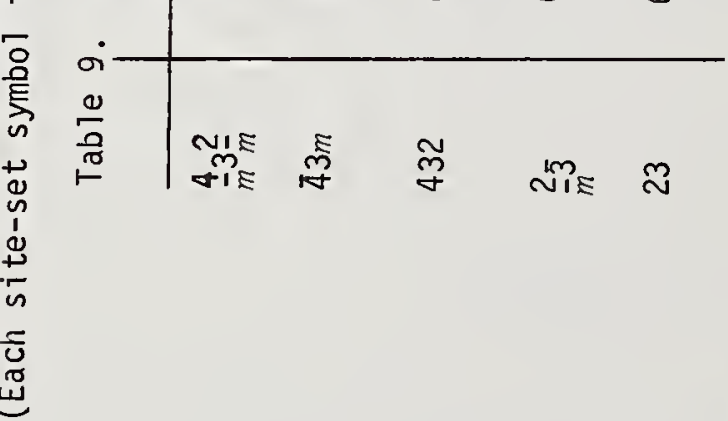

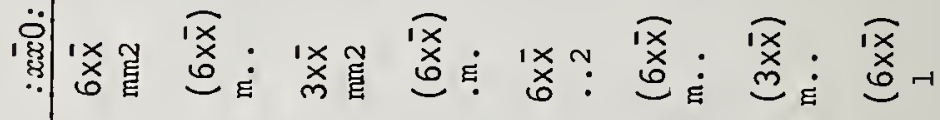

ดุ $ก$ ก

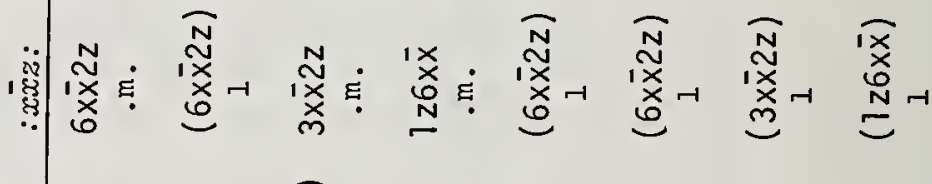

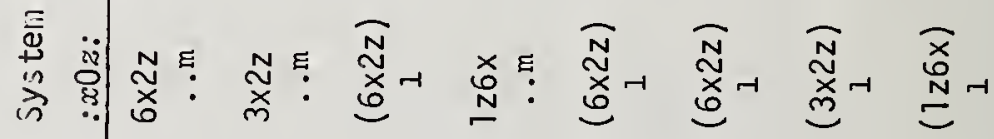

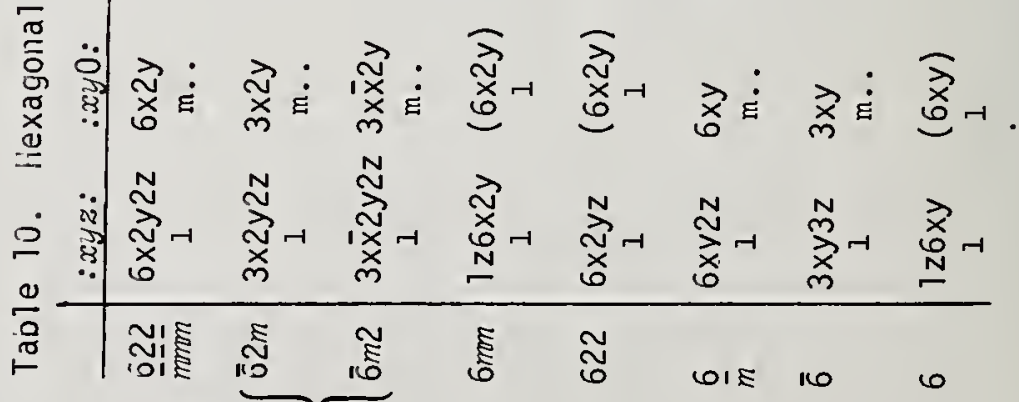




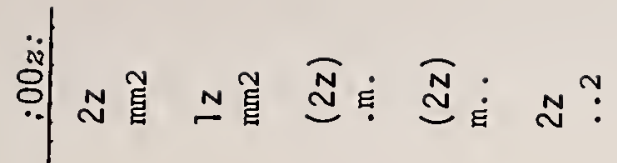

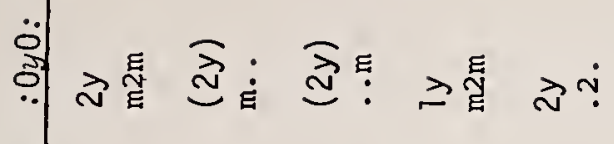

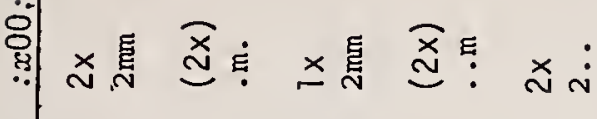

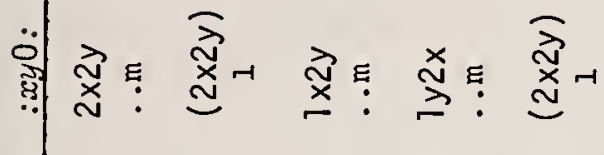

焉

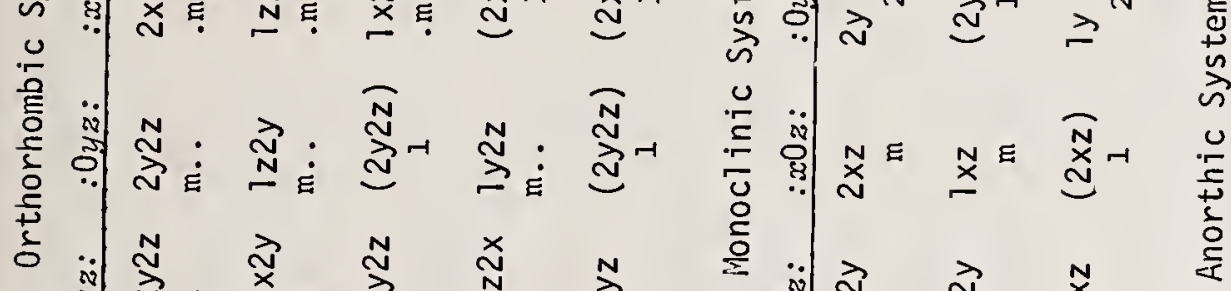

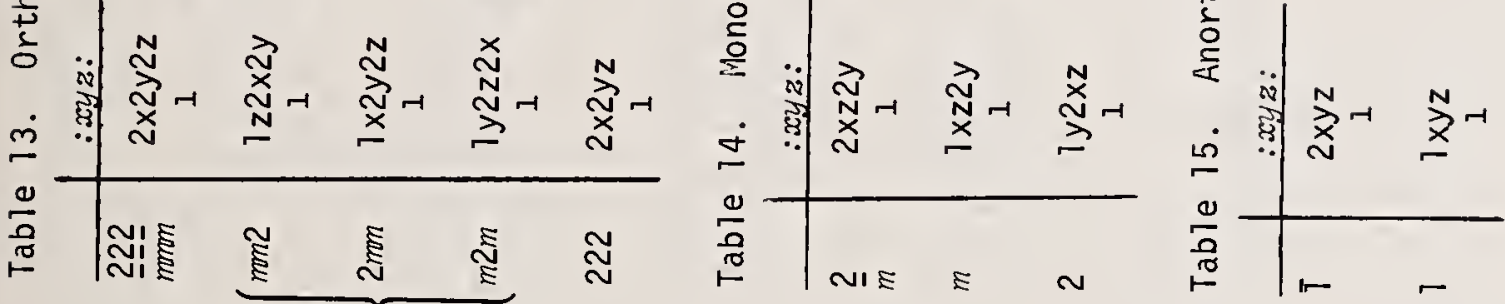

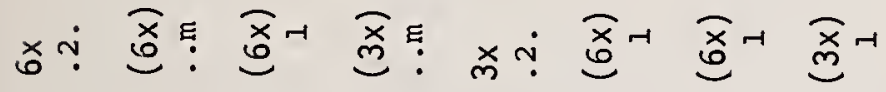

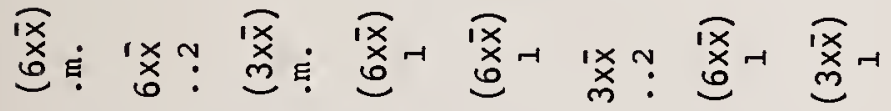

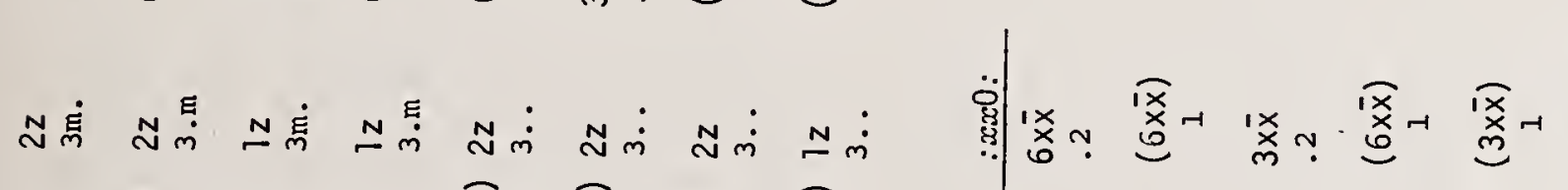

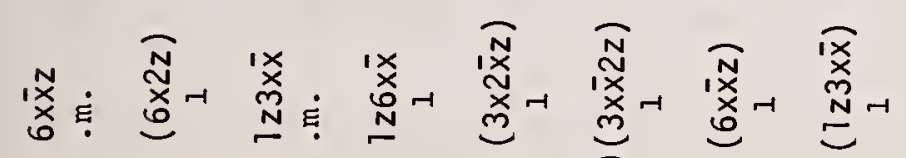

蒙

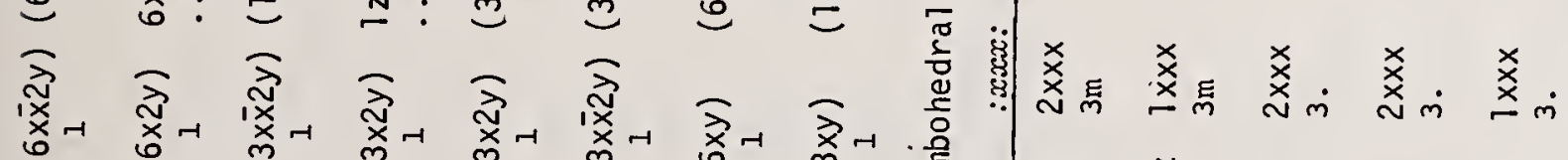

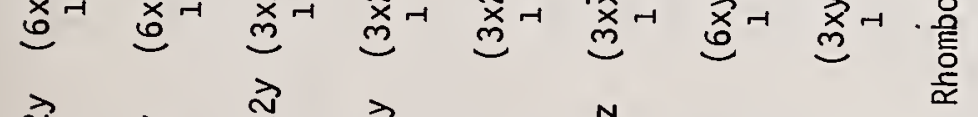

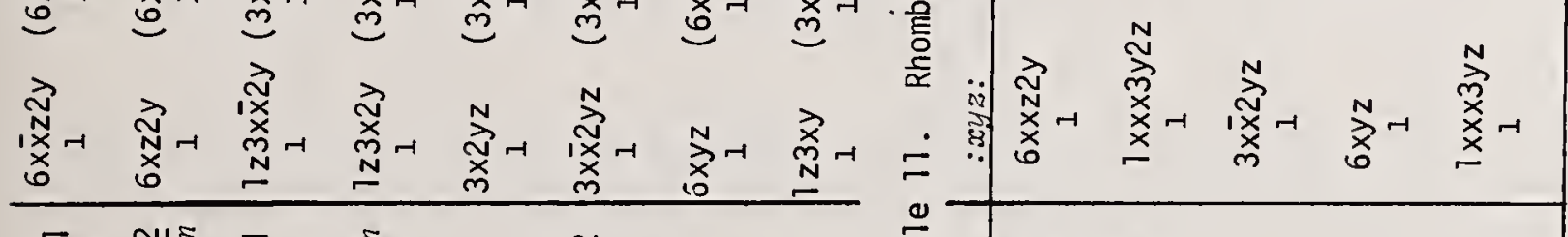

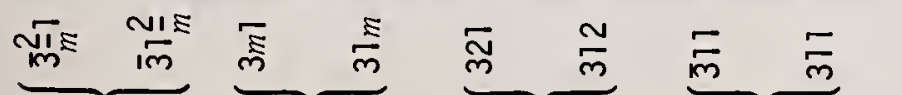

总

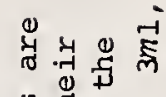

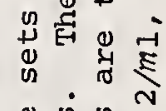
g. का.

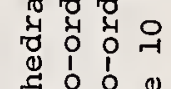

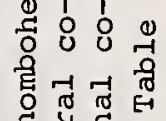

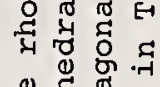

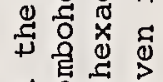
न व्वृत के

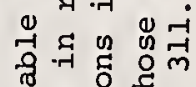
⿶凵

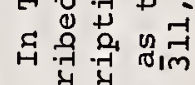
- $\rightarrow$ : 


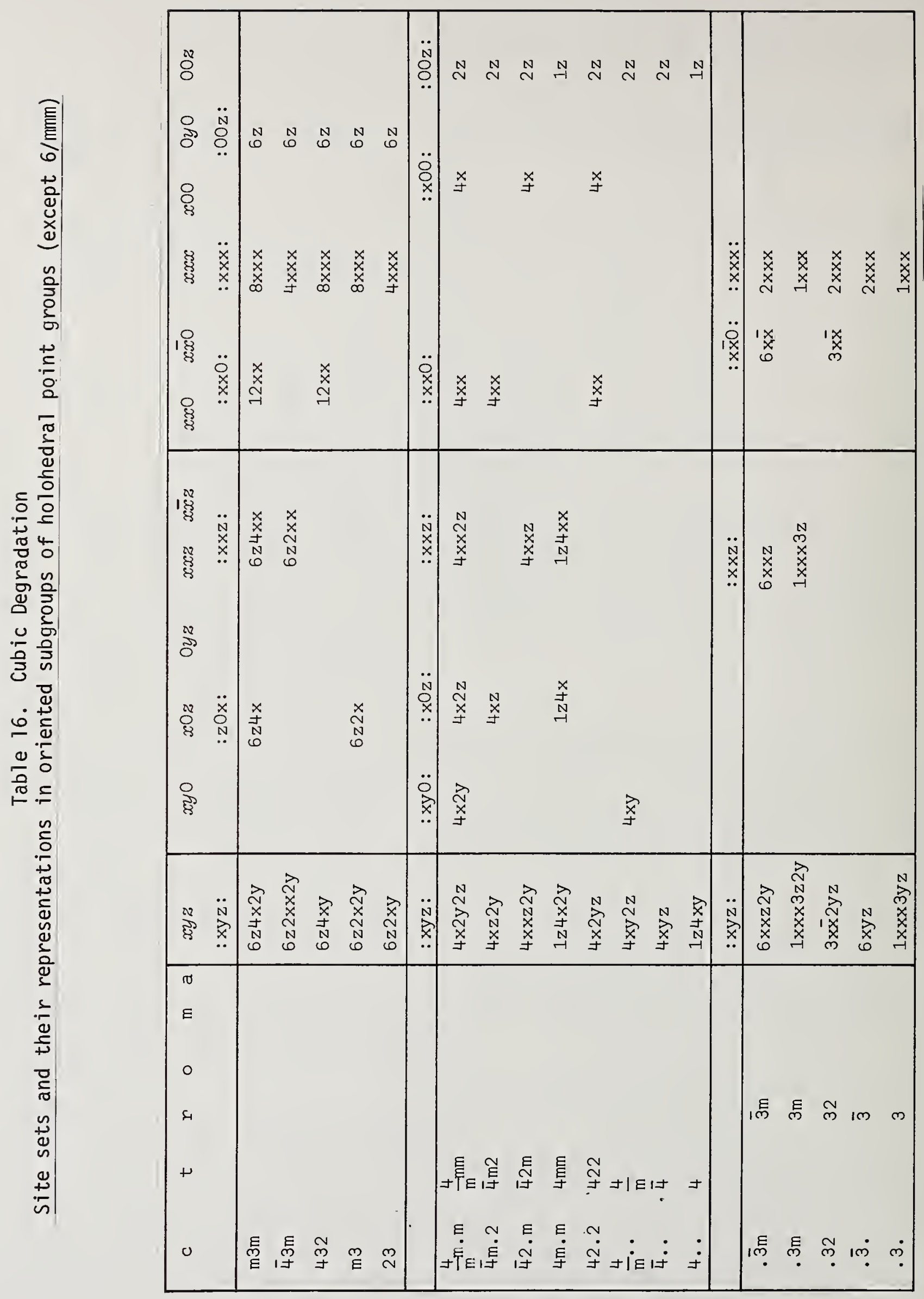




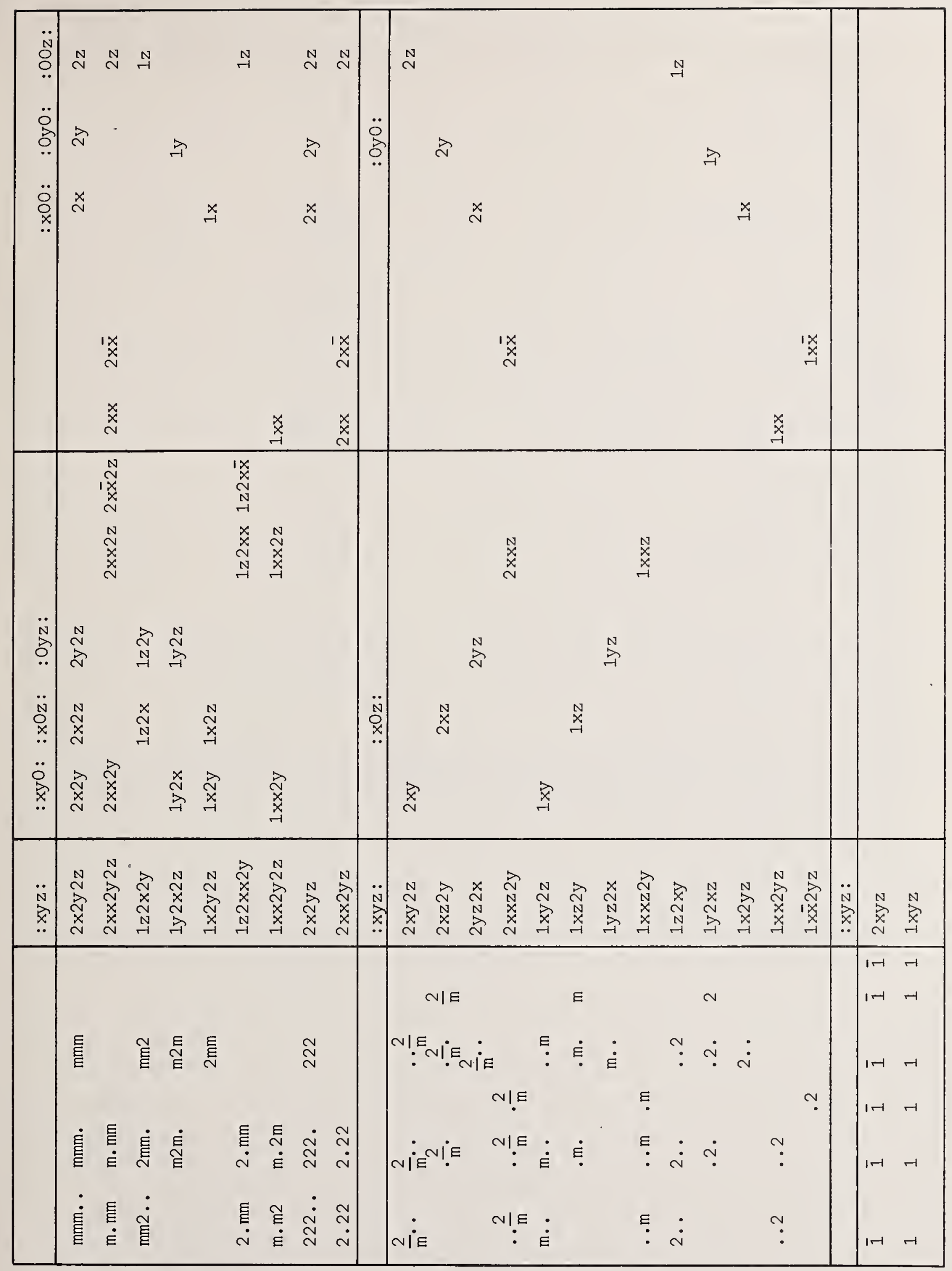




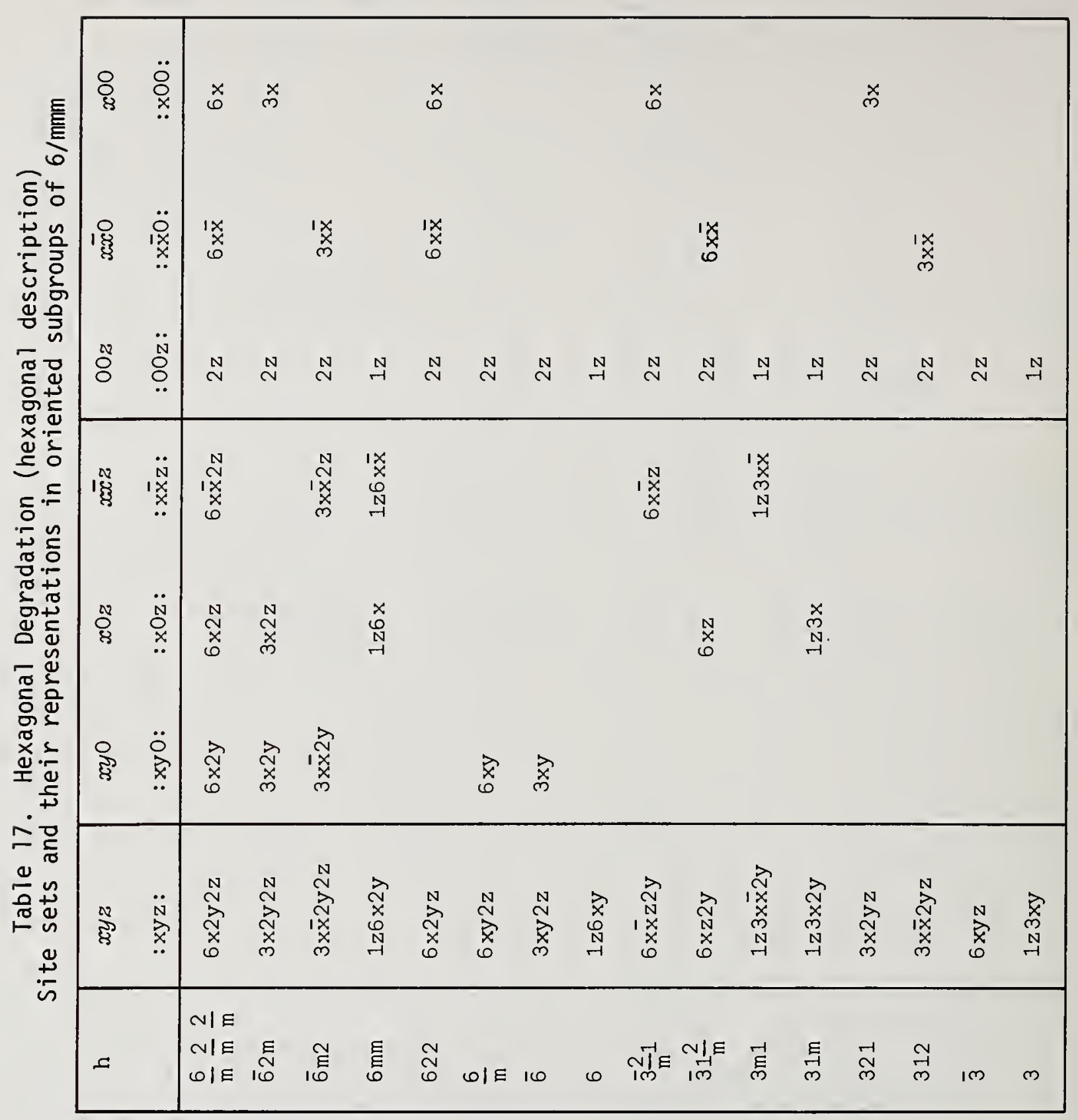




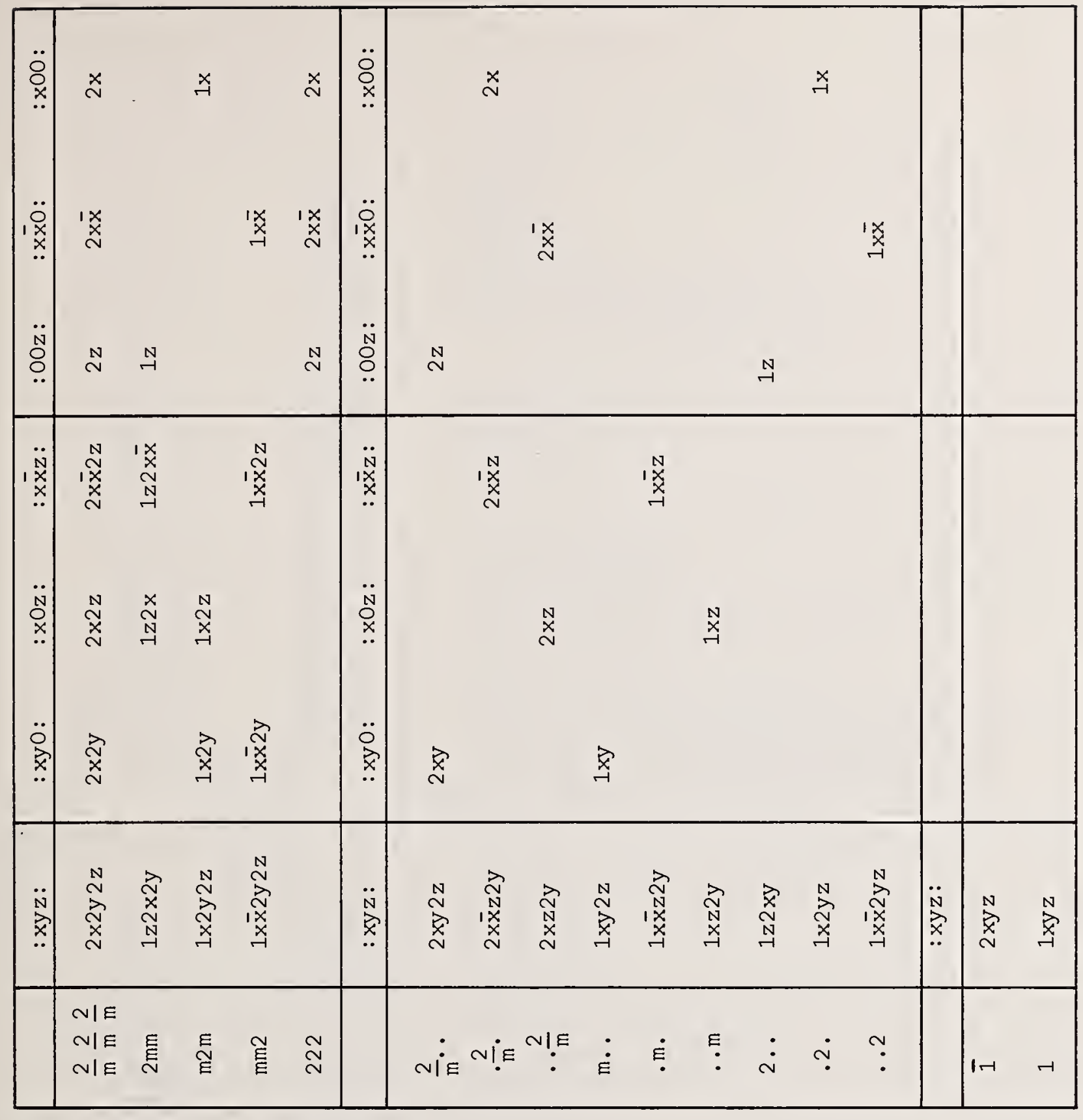



Table 18: Cubic system

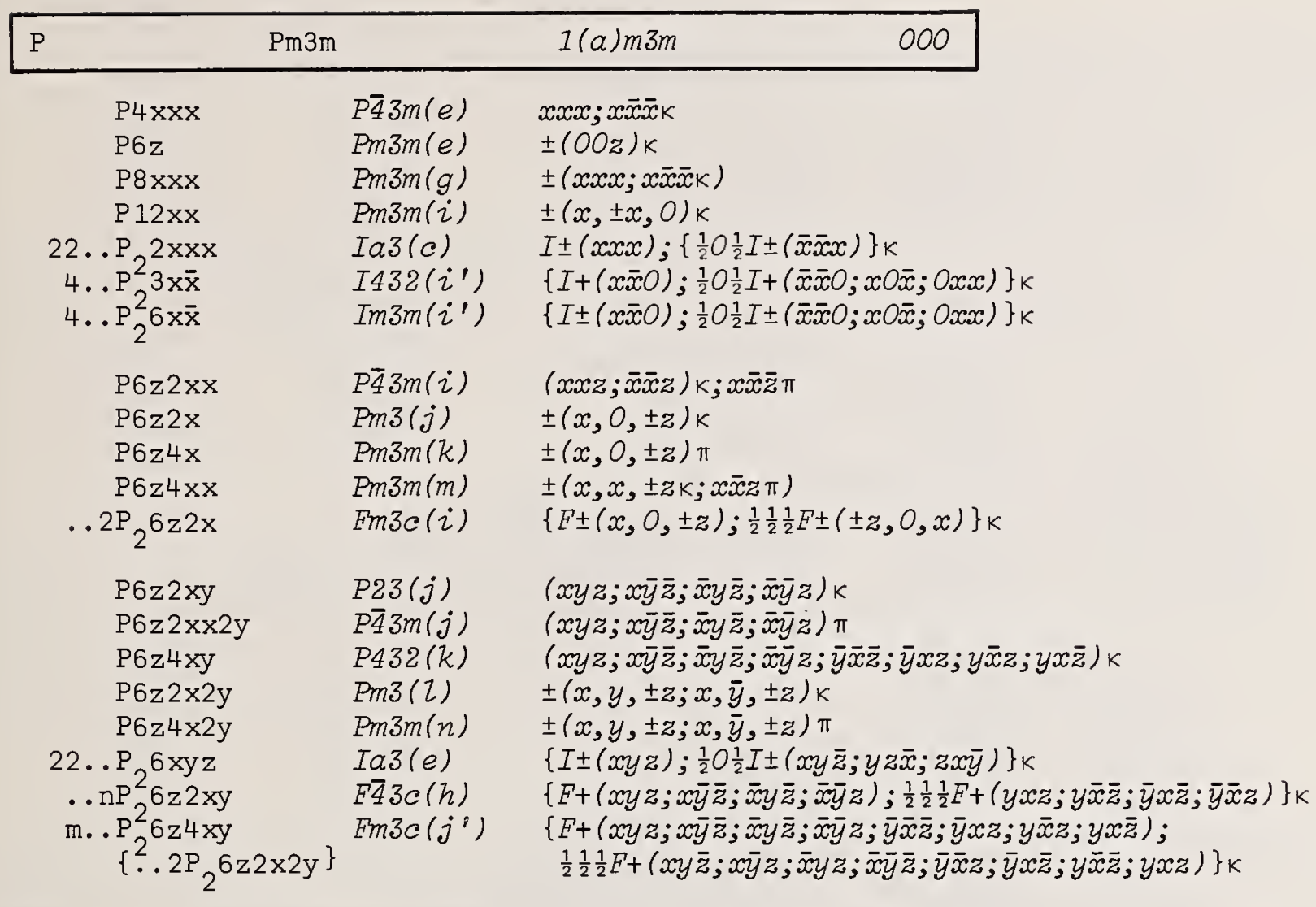

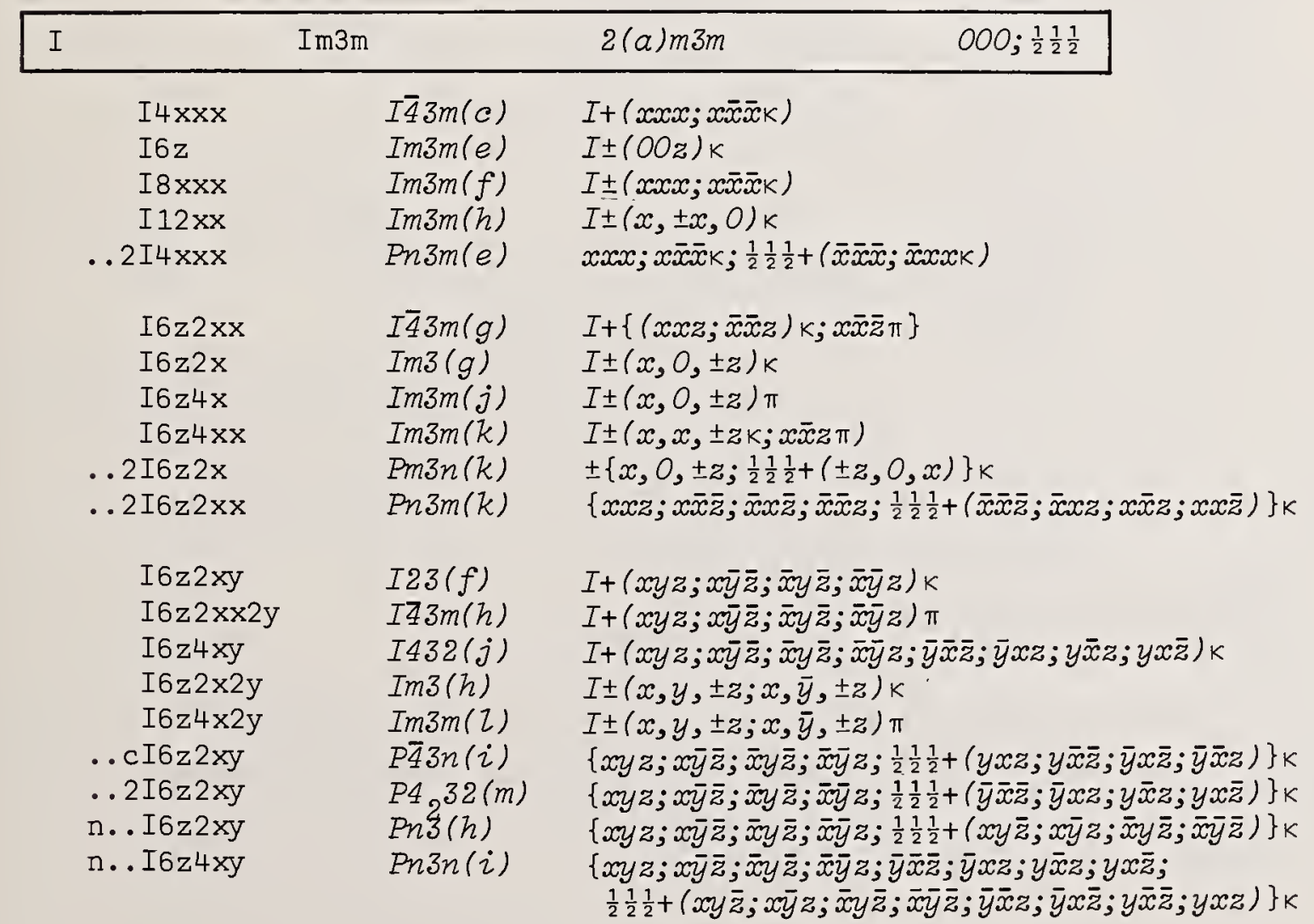


$\ldots 2 I 6 z 2 x 2 y$

. . 2I6z2xx2y

d. $2 I_{2} 6 z 2 x y$
$\operatorname{Pm} 3 n(Z)$

$\operatorname{Pn} 3 m(Z)$

$F d 3 c(h)$ $\pm\left\{x, y, \pm z ; x, \bar{y}, \pm z ; \frac{1}{2} \frac{1}{2} \frac{1}{2}+(y, x, \pm z ; \bar{y}, x, \pm z)\right\}_{k}$ $\left\{x y z ; x \bar{y} \bar{z} ; \bar{x} y \bar{z} ; \bar{x} \bar{y} z ; \frac{1}{2} \frac{1}{2} \frac{1}{2}+(\bar{x} \bar{y} \bar{z} ; \bar{x} y z ; x \bar{y} z ; x y \bar{z})\right\} \pi$ $F+\left\{x y z ; x \bar{y} \bar{z} ; \bar{x} y \bar{z} ; \bar{x} \bar{y} z ; \frac{1}{4} \frac{1}{4} \frac{1}{4}+(\bar{y} \bar{x} \bar{z} ; \bar{y} x z ; y \bar{x} z ; y x \bar{z}) ;\right.$

$\left.\frac{1}{2} \frac{1}{2} \frac{1}{2}+(y x z ; y \bar{x} \bar{z} ; \bar{y} x \bar{z} ; \bar{y} \bar{x} z) ; \frac{3}{4} \frac{3}{4} \frac{3}{4}+(\bar{x} \bar{y} \bar{z} ; \bar{x} y z ; x \bar{y} z ; x y \bar{z})\right\}_{k}$

\begin{tabular}{|llll|}
\hline $\mathrm{J}$ & $\mathrm{Pm} 3 \mathrm{~m}$ & $3(\mathrm{c}) 4 / \mathrm{mm} \cdot \mathrm{m}$ & $\frac{1}{2} \mathrm{O} \frac{1}{2} \mathrm{~K}$ \\
\hline
\end{tabular}

.3. $\mathrm{J} 2 \mathrm{x}$

$\operatorname{Pm} 3(f) \quad \pm\left(\frac{1}{2}+x, 0, \frac{1}{2}\right) k$

$.3 . \mathrm{J} 4 \mathrm{x}$

$\operatorname{Pm} 3 m(h) \quad \pm\left(\frac{1}{2}+x, 0, \frac{1}{2}\right) \pi$

\begin{tabular}{|c|c|c|}
\hline $\mathrm{F}$ & $\mathrm{Fm} 3 \mathrm{~m}$ & $000 ; \frac{1}{2} O \frac{1}{2} K$ \\
\hline$F 4 x \times x$ & $F \overline{4} 3 m(e)$ & $F+(x x x ; x \bar{x} \bar{x} \kappa)$ \\
\hline F6z & $\operatorname{Em} 3 m(e)$ & $F \pm(O O z) K$ \\
\hline F8xxx & $\operatorname{Em} 3 m(f)$ & $F \pm(x x x ; x \bar{x} \bar{x} \kappa)$ \\
\hline $\mathrm{F} 12 \mathrm{xx}$ & $\operatorname{Em} 3 m(h)$ & $F \pm(x, \pm x, 0) k$ \\
\hline bc..F $2 x \times x$ & $\operatorname{Pa3}(\mathrm{c})$ & $\pm(x x x) ;\left\{\frac{1}{2} O \frac{1}{2} \pm(x x \bar{x})\right\} \kappa$ \\
\hline$\underline{4}, \ldots F 3 x \bar{x}$ & $P 4_{2} 32\left(k^{\prime}\right)$ & $\left\{x \bar{x} O ; \frac{1}{2} O \frac{1}{2}+(\bar{x} \bar{x} O ; x O \bar{x} ; O x x)\right\}_{\kappa}$ \\
\hline$\overline{4}^{2} . F 6 \times \bar{x}$ & $\operatorname{Pn} 3 m\left(i^{\prime}\right)$ & $\pm\left\{x \bar{x} O ; \frac{1}{2} O \frac{1}{2}+(\bar{x} \bar{x} O ; x O \bar{x} ; O x x)\right\}_{\kappa}$ \\
\hline$\overline{4} 2 \ldots F_{2} 3 x \bar{x}$ & $F d 3 c\left(g^{\prime}\right)$ & $\begin{array}{l}F+\left\{x \bar{x} O ; \frac{1}{4} O \frac{1}{4}+(\bar{x} \bar{x} O ; x O \bar{x} ; O x x) ; \frac{1}{2} \frac{1}{2} \frac{1}{2}+(\bar{x} x O) ;\right. \\
\left.\quad \frac{1}{4} \frac{1}{2} \frac{1}{4}+(x x O ; \bar{x} O x ; O \bar{x} \bar{x})\right\}_{K}\end{array}$ \\
\hline F6z2xx & $F \overline{4} 3 m(h)$ & $F+\{(x x z ; \bar{x} \bar{x} z) \kappa ; x \bar{x} \bar{z} \pi\}$ \\
\hline F6z2x & $\operatorname{Fm} 3(h)$ & $F \pm(x, 0, \pm z) \kappa$ \\
\hline F6z4x & $\operatorname{Em} 3 m(j)$ & $F \pm(x, 0, \pm z) \pi$ \\
\hline F6 $z 4 x x$ & $\operatorname{Em} 3 m(k)$ & $F \pm(x, x, \pm z \kappa ; x \bar{x} z \pi)$ \\
\hline F6z2xy & $F 23(h)$ & $F+(x y z ; x \bar{y} \bar{z} ; \bar{x} y \bar{z} ; \bar{x} \bar{y} z) k$ \\
\hline F6 z $2 x \times 2 y$ & $F \overline{4} 3 m(i)$ & $F+(x y z ; x \bar{y} \bar{z} ; \bar{x} y \bar{z} ; \bar{x} \bar{y} z) \pi$ \\
\hline F6z $4 x y$ & $F 432(j)$ & $F+(x y z ; x \bar{y} \bar{z} ; \bar{x} y \bar{z} ; \bar{x} \bar{y} z ; \bar{y} \bar{x} \bar{z} ; \bar{y} x z ; y \bar{x} z ; y x \bar{z}) k$ \\
\hline F $6 z 2 x 2 y$ & $\operatorname{Em} 3(i)$ & $F \pm(x, y, \pm z ; x, \bar{y}, \pm z)_{\kappa}$ \\
\hline$F 6 z 4 x 2 y$ & $\operatorname{Em} 3 m(Z)$ & $F \pm(x, y, \pm z ; x, \bar{y}, \pm z) \pi$ \\
\hline bc..F6xyz & $\operatorname{Pa3}(d)$ & $\pm\left\{x y z ; \frac{1}{2} O \frac{1}{2}+(x y \bar{z} ; z x \bar{y} ; y z \bar{x})\right\}_{\kappa}$ \\
\hline
\end{tabular}

\begin{tabular}{|llll|}
\hline${ }^{+} \mathrm{P}$ & $\mathrm{P}_{3} 3^{32}$ & $4(a) .32$ & $\frac{1}{8} \frac{1}{8} \frac{1}{8} ; \frac{7}{8} \frac{5}{8} \frac{3}{8} \mathrm{~K}$ \\
\hline
\end{tabular}

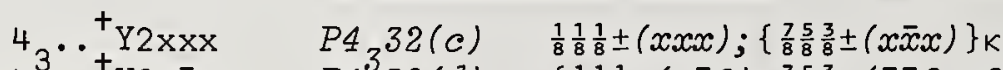

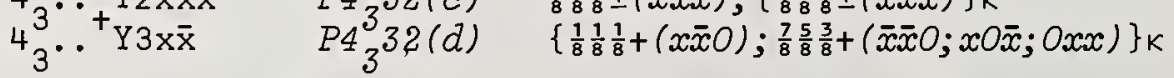

${ }_{3}{ }^{+}+{ }^{+} \mathrm{Y} 3 \mathrm{x} \overline{\mathrm{x}} 2 \mathrm{yz} \quad P 4{ }_{3} 32(e) \quad\left\{\frac{1}{8} \frac{1}{8} \frac{1}{8}+(x y z ; \bar{y} \bar{x} \bar{z}) ; \frac{7}{8} \frac{5}{8} \frac{3}{8}+(\bar{x} y \bar{z} ; \bar{z} x \bar{y} ; \bar{y} z \bar{x} ; y \bar{x} z ; z \bar{y} x ; x \bar{z} y)\right\} \kappa$

\begin{tabular}{|c|c|c|}
\hline $\operatorname{Pm} 3 n$ & $6(c) \overline{4} m .2$ & $\pm\left(O \frac{1}{2} \frac{1}{4}\right) k$ \\
\hline
\end{tabular}
.3. $\mathrm{W} 2 \mathrm{z}$
$\{.3 . J * 2 z\}$
$\operatorname{Pm} 3 n(g) \quad \pm\left(0, \frac{1}{2}, \frac{1}{4} \pm z\right) k$
.3. $14 \mathrm{xx}$
$\operatorname{Pm} 3 n(j) \quad \pm\left(x, \frac{1}{2} \pm x, \frac{1}{4} ; \bar{x}, \frac{1}{2} \pm x, \frac{1}{4}\right)_{k}$

\begin{tabular}{|c|c|c|c|}
\hline$J^{*}$ & $\operatorname{Im} 3 \mathrm{~m}$ & $6(\mathrm{~b}) 4 / \mathrm{mm} \cdot \mathrm{m}$ & $I+\left(\frac{1}{2} O \frac{1}{2}\right) K$ \\
\hline
\end{tabular}

-3. $J^{*} 2 \mathrm{x} \quad \operatorname{Im} 3(e) \quad I \pm\left(\frac{1}{2}+x, 0, \frac{1}{2}\right) K$




\begin{tabular}{|llll|}
\hline$D$ & Fd3m & $8(a) \overline{4} 3 m$ & $F+\left(000 ; \frac{1}{4} \frac{1}{4} \frac{1}{4}\right)$ \\
\hline
\end{tabular}

\begin{tabular}{|c|c|c|}
\hline $\begin{array}{c}D 6 z \\
.2 D 4 x \times x\end{array}$ & $\begin{array}{l}F d 3 m(f) \\
F d 3 m(e)\end{array}$ & $\begin{array}{l}F+\left(0,0, \pm z ; \frac{1}{4}, \frac{1}{4}, \frac{1}{4} \pm z\right) k \\
F+\left\{x x x ; x \bar{x} \bar{x} \kappa ; \frac{1}{4} \frac{1}{4}+(\bar{x} \bar{x} \bar{x} ; \bar{x} x x \kappa)\right\}\end{array}$ \\
\hline. $.2 \mathrm{D} 6 \mathrm{z} 2 \mathrm{xx}$ & $F d 3 m(g)$ & $F+\left\{x x z ; x \bar{x} \bar{z} ; \bar{x} x \bar{z} ; \bar{x} \bar{x} z ; \frac{1}{4} \frac{1}{4} \frac{1}{4}+(\bar{x} \bar{x} \bar{z} ; \bar{x} x z ; x \bar{x} z ; x x \bar{z})\right\}_{k}$ \\
\hline $\begin{array}{l}.2 \mathrm{D} 6 \mathrm{z} 2 \mathrm{xy} \\
\mathrm{d} . \mathrm{D} 6 \mathrm{z} 2 \mathrm{xy} \\
. \mathrm{D} \mathrm{D} 6 \mathrm{z} 2 \mathrm{xx} 2 \mathrm{y}\end{array}$ & $\begin{array}{l}F 432(h) \\
F d 3(g) \\
F d 3 m(i)\end{array}$ & 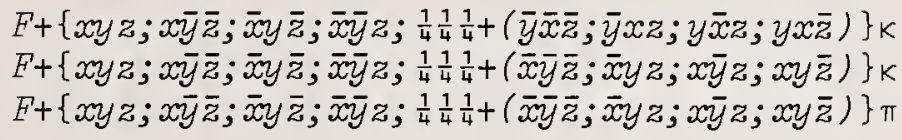 \\
\hline
\end{tabular}

\begin{tabular}{|llll|}
\hline${ }^{+}{ }^{*}$ & ${ }^{14}{ }_{1} 32$ & $8(a) .32$ & $I+\left(\frac{1}{8} \frac{1}{8} \frac{1}{8} ; \frac{75}{8} \frac{5}{8} \mathrm{~K}\right)$ \\
\hline
\end{tabular}

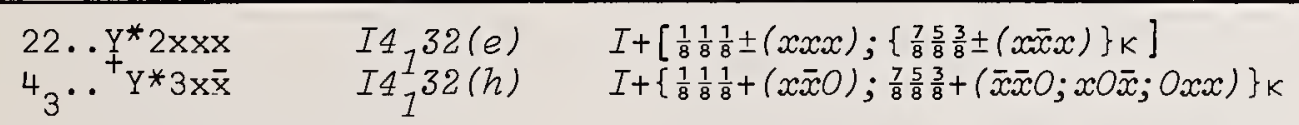

$22 . . Y * 3 x \bar{x} 2 y z \quad I 4{ }_{1} 32(i) \quad I+\left\{\frac{1}{8} \frac{1}{8} \frac{1}{8}+(x y z ; \bar{y} \bar{x} \bar{z}) ; \frac{7}{8} \frac{5}{8} \frac{3}{8}+(\bar{x} y \bar{z} ; \bar{z} x \bar{y} ; \bar{y} z \bar{x} ; y \bar{x} z ; z \bar{y} x ; x \bar{z} y)\right\}_{K}$

\begin{tabular}{|llll|}
\hline $\mathrm{W}^{*}$ & $\mathrm{Im} 3 \mathrm{~m}$ & $12(\mathrm{~d}) \overline{4} \mathrm{~m} .2$ & $I \pm\left(O \frac{1}{2} \frac{1}{4}\right) \mathrm{K}$ \\
\hline $\mathrm{S}$ & $\mathrm{I \overline {4 }} 3 \mathrm{~d}$ & $12(\mathrm{a}) \overline{4} .$. & $I+\left(O \frac{1}{4} \frac{3}{8} ; O \frac{3}{4} \frac{1}{8}\right) \mathrm{K}$ \\
\hline
\end{tabular}

.3. $22 \mathrm{z} \quad I \overline{4} 3 d(d) \quad I+\left(0, \frac{1}{4}, \frac{3}{8} \pm z ; 0, \frac{3}{4}, \frac{1}{8} \pm z\right)_{K}$

.3dS4xyz $I \overline{4} 3 d(e) \quad I+\left\{0 \frac{1}{4} \frac{3}{8}+(x y z ; \bar{x} \bar{y} z ; y \bar{x} \bar{z} ; \bar{y} x \bar{z}) ; O \frac{3}{4} \frac{1}{8}+(y x z ; \bar{y} \bar{x} z ; x \bar{y} \bar{z} ; \bar{x} y \bar{z})\right\} k$

\begin{tabular}{|llll|}
\hline${ }^{\mathrm{V}}$ & $\mathrm{I}_{4} 1^{32}$ & $12(\mathrm{c}) 2.22$ & $I+\left(0 \frac{1}{4} \frac{1}{8} ; O \frac{3}{4} \frac{3}{8}\right) \mathrm{K}$ \\
\hline
\end{tabular}

.3. v2z

$I 4 I_{1}^{32(f) \quad I+\left(0, \frac{1}{4}, \frac{1}{8} \pm 2 ; 0, \frac{3}{4}, \frac{3}{8} \pm 2\right) \mathrm{K}}$

\begin{tabular}{|llll|}
\hline $\mathrm{T}$ & $\mathrm{Fd} 3 \mathrm{~m}$ & $16(c) . \overline{3} \mathrm{~m}$ & $F+\left(\frac{1}{8} \frac{1}{8} \frac{1}{8} ; \frac{3}{8} \frac{1}{8} \frac{3}{8} \mathrm{~K}\right)$ \\
\hline
\end{tabular}

\begin{tabular}{|c|c|c|}
\hline $\begin{array}{r}22 \ldots \text { T } 3 \times \bar{x} \\
\overline{4} \ldots \text { T } 6 x \bar{x}\end{array}$ & $\begin{array}{l}F 432(g) \\
F d 3 m(h)\end{array}$ & $\begin{array}{l}F+\left\{\frac{1}{8} \frac{1}{8}\right. \\
F+\left\{\frac{1}{8}\right.\end{array}$ \\
\hline
\end{tabular}

\begin{tabular}{|c|c|c|c|}
\hline$Y^{* *}$ & Ia3d & $16(b) .32$ & $I \pm\left(\frac{1}{8} \frac{1}{8} \frac{1}{8} ; \frac{75}{8} \frac{3}{8} K\right)$ \\
\hline
\end{tabular}

\begin{tabular}{|c|c|c|}
\hline \multirow{2}{*}{$\begin{array}{c}\overline{4} \ldots Y^{* *} 2 x x x \\
\{\overline{4} . . I 22 x x x\} \\
\overline{4} a . . Y^{* *} 3 x \bar{x}\end{array}$} & $\operatorname{Ia} 3 d(e)$ & $I \pm\left[\frac{1}{8} \frac{1}{8} \frac{1}{8} \pm(x x x) ;\left\{\frac{7}{8} \frac{5}{8} \frac{3}{8} \pm(x \bar{x} x)\right\}_{K}\right]$ \\
\hline & $\operatorname{Ia} 3 d(g)$ & $I \pm\left\{\frac{1}{8} \frac{1}{8} \frac{1}{8}+(x \bar{x} O) ; \frac{7}{8} \frac{5}{8} \frac{3}{8}+(\bar{x} \bar{x} O ; x O \bar{x} ; O x x)\right\}_{K}$ \\
\hline $\begin{aligned} \overline{4} a . . & y^{* *} 3 x \bar{x} 2 y z \\
& \left\{\overline{4} .2 \mathrm{I}{ }_{2} 6 \mathrm{xyz}\right\}\end{aligned}$ & $\operatorname{Ia} 3 d(h)$ & $I \pm\left\{\frac{1}{1} \frac{1}{8} \frac{1}{8}+(x y z ; \bar{y} \bar{x} \bar{z}) ; \frac{75}{8} \frac{3}{8}+(\bar{x} y \bar{z} ; \bar{z} x \bar{y} ; \bar{y} z \bar{x} ; y \bar{x} z ; z \bar{y} x ; x \bar{z} y)\right\}_{k}$ \\
\hline
\end{tabular}

\begin{tabular}{|c|c|c|c|}
\hline$s^{*}$ & Ia3d & $24(d) \overline{4} .$. & $I \pm\left(O \frac{1}{4} \frac{3}{8} ; O \frac{3}{4} \frac{1}{8}\right) \mathrm{K}$ \\
\hline
\end{tabular}
.3. $5 * 2 \mathrm{z}$
$\{.3 . V * 2 z\}$
$\operatorname{Ia} 3 d(f)$
$I \pm\left(0, \frac{1}{4}, \frac{3}{8} \pm z ; 0, \frac{3}{4}, \frac{1}{8} \pm z\right)_{K}$ 


\begin{tabular}{|c|c|c|c|}
\hline$V^{*}$ & $\operatorname{Ia} d \mathrm{~d}$ & $24(c) 2.22$ & $I \pm\left(O \frac{1}{4} \frac{1}{8} ; O \frac{3}{4} \frac{3}{8}\right) \mathrm{K}$ \\
\hline
\end{tabular}

\begin{tabular}{|c|c|c|c|}
\hline${ }^{2}{ }_{1}{ }^{2} \ldots F Y 1 x x x$ & $\mathrm{P}_{1}{ }_{1}^{3}$ & $4(a) .3$ & $x x x ;\left\{\frac{1}{2} O \frac{1}{2}+(\bar{x} \bar{x} x)\right\}_{k}$ \\
\hline
\end{tabular}

${ }_{2}{ }_{1}{ }_{1} \ldots$ FY $1 \mathrm{xxx} 3 \mathrm{yz} \quad P 2{ }_{1} 3(b) \quad\left\{x y z ; \frac{1}{2} O \frac{1}{2}+(\bar{x} \bar{y} z ; \bar{y} \bar{z} x ; \bar{z} \bar{x} y)\right\}_{k}$

\begin{tabular}{|c|c|c|c|}
\hline${ }^{2}{ }_{1}{ }_{1} \cdots P_{2} Y^{*} 1 x x x$ & $\operatorname{I2}_{1} 3$ & $8(a) .3$ & $I+\left[x x x ;\left\{\frac{1}{2} O \frac{1}{2}+(\bar{x} \bar{x} x)\right\}_{k}\right]$ \\
\hline
\end{tabular}

${ }^{2}{ }_{1}{ }_{1} \ldots \mathrm{P}_{2} \mathrm{Y}^{*} 1 \times x \times 3 y z \quad I 2{ }_{1} 3(c) \quad I+\left\{x y z ; \frac{1}{2} O \frac{1}{2}+(\bar{x} \bar{y} z ; \bar{y} \bar{z} x ; \bar{z} \bar{x} y)\right\}_{K}$

\begin{tabular}{|c|c|c|c|}
\hline $21^{3 . S V 1 z}$ & $\operatorname{I2}_{1}^{3}$ & $12(b) 2 \ldots$ & $\left(0, \frac{1}{4}, \frac{3}{8}+z ; 0, \frac{3}{4}, \frac{1}{8}-z\right) k$ \\
\hline
\end{tabular}

\begin{tabular}{|c|c|c|c|}
\hline$\overline{4} \ldots I_{2} Y^{* *} 1 \mathrm{xXX}$ & $1 \overline{4} 3 \mathrm{~d}$ & $16(c) .3$. & $\begin{aligned} I+ & {\left[x x x ; \frac{1}{4} \frac{1}{4}+(x x x) ;\right.} \\
& \left.\left\{\frac{1}{2} O \frac{1}{2}+(\bar{x} \bar{x} x) ; \frac{3}{4} \frac{1}{4} \frac{3}{4}+(x \bar{x} \bar{x})\right\} K\right]\end{aligned}$ \\
\hline
\end{tabular}

\begin{tabular}{|llll}
\hline$\cdot \overline{3} \cdot \mathrm{J}_{2} \mathrm{~S}^{*} \mathrm{~V}^{*} 1 \mathrm{x}$ & $\mathrm{Ia} 3$ & $24(\mathrm{~d}) 2 .$. & $I \pm\left(\frac{1}{4}+x, 0, \frac{1}{4} ; \frac{1}{4}+x, \frac{1}{2}, \frac{3}{4}\right) \mathrm{K}$ \\
\hline
\end{tabular}

Table 19: Hexagonal System

(and rhombohedral system in hexagonal description)

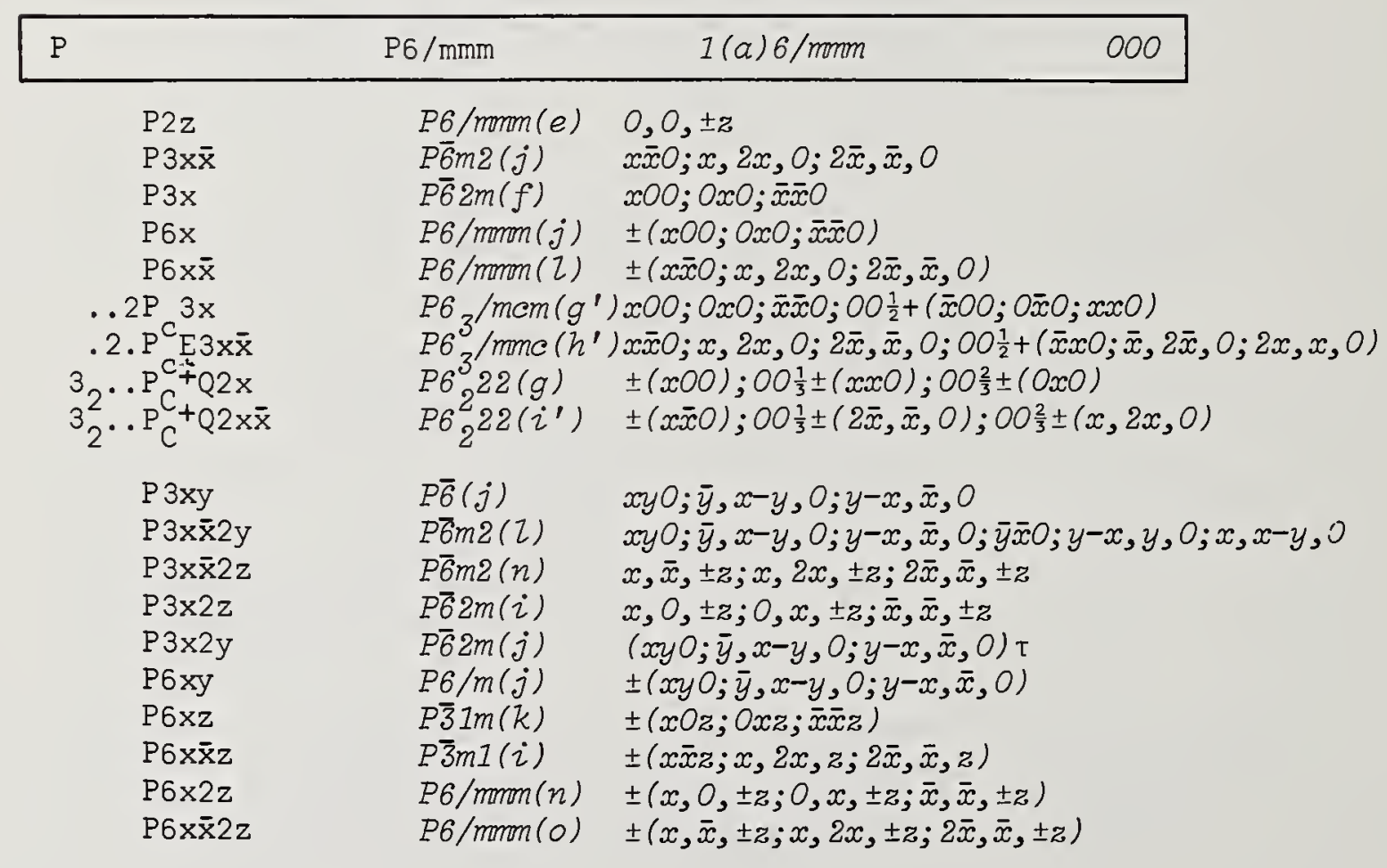


P6x2y

. 2P $3 x y$

. 2. $P^{C} E 3 x y$

2... $P_{E 3 x y}$

$3_{2}^{1} \cdot P_{C}^{C+Q 2 x y}$

2. $P^{6} 6 x y$

. $2 \mathrm{P}^{\mathrm{C}} 3 \mathrm{x} 2 \mathrm{y}$

. $2 \mathrm{P}^{\mathrm{C}} 3 \mathrm{x} 2 \mathrm{z}$

$\{\mathrm{c}$. P6xz $\}$

.2. $P_{c} E 3 x \bar{x} 2 y$

.2.P E3x $\bar{x} 2 z$

$\{\stackrel{c}{\mathrm{~m}} . . \mathrm{P} 6 \times \bar{x} z\}$

P3xy $2 z$

P $3 x \bar{x} 2 y z$

P $3 x 2 y z$

P6xyz

P $3 x \bar{x} 2 y 2 z$

P. $3 x 2 y 2 z$

P6x2yz

P6xy 2z

P6xz2y

P $6 x \bar{x} z 2 y$

P6x2y $2 z$

. 2P $3 x y 2 z$

$\{\mathrm{c} . . P 3 x \bar{x} 2 \mathrm{yz}\}$

.2. P E3xy $2 z$

$\{\stackrel{c}{m}$.. P 3x2yz $\}$

${ }_{2} \ldots \mathrm{P}_{\mathrm{C}} \mathrm{F}_{\mathrm{Q} 2} \mathrm{x}_{2 \mathrm{yz}}$

. 2P $3 x 2 y z$

$\{$ C. $2 . P$ E $3 x \bar{x} 2 y z\}$

$2 \ldots P$ E $3 x y$ y $2 z$

$1\left\{\stackrel{c}{m} . . P_{c} 6 x y z\right\}$

..cP E3x $2 y z$

$\{$ C $2 P$ S $6 x y z\}$

.n. $\mathrm{P} 3 \mathrm{x} 2 \mathrm{yz}$

$\left\{\stackrel{C}{C} \cdot P_{6 x y z}\right\}$

m. P $6 \times 2$ yz

$\left\{{ }^{C} 2 . P c_{c} 6 x y 2 z\right\}$

. 2 P $3 x 2 y 2 z$

$\left\{\stackrel{\mathrm{c}}{\mathrm{m}} . \mathrm{P}_{\mathrm{c}} 6 \mathrm{xz} 2 \mathrm{y}\right\}$

. 2. P E3x 2 2y $2 z$

$\left\{\stackrel{c}{m} \ldots P_{c} \delta x \bar{x} z 2 y\right\}$
P6/mmm ( $p)$

Pद्6c2 (k')

$P \overline{6} 2 c\left(h^{\prime}\right)$

$P 6_{3} / m\left(h^{\prime}\right)$

$P 6_{2}^{3}\left(c^{\prime}\right)$

P64mec (Z)

$P 6_{3} / \operatorname{mcm}\left(j^{\prime}\right)$

$P 6_{3}^{3} / \operatorname{mcm}\left(k^{\prime}\right)$

$P 6_{3} / \operatorname{moc}\left(j^{\prime}\right)$

$P 6_{3} / \operatorname{mmc}\left(k^{\prime}\right)$

$P \overline{6}(Z)$

P312(2)

P321 $(\mathrm{g})$

$P \overline{3}(g)$

Pद्6m2 (o)

$P \overline{6} 2 m(Z)$

$P 622(n)$

$P 6 / m(Z)$

$P \overline{3} \operatorname{Im}(\mathrm{Z})$

$P \overline{3} m 1(j)$

$P 6 / \operatorname{mrm}(r)$

Pद्6c2( (2')

$P \overline{6} 2 c\left(i^{\prime}\right)$

$P 62^{22(k)}$

${ }^{P 6}{ }_{3}^{22(i)}$

$P 6_{3} / m\left(i^{\prime}\right)$

$P \overline{3} 1 c\left(i^{\prime}\right)$

$P \overline{3} c 1\left(g^{\prime}\right)$

$P 6 / \operatorname{mec}\left(m^{\prime}\right)$

$\mathrm{P6}_{3} / \mathrm{mem}\left(Z^{\prime}\right)$

$P 6_{3} /$ mme (2') $\pm(x y 0 ; \bar{y}, x-y, 0 ; y-x, \bar{x}, 0) \tau$

$x y 0 ; \bar{y}, x-y, 0 ; y-x, \bar{x}, 0 ; 00 \frac{1}{2}+(\bar{y} \bar{x} 0 ; y-x, y, 0 ; x, x-y, 0)$

xy $0 ; \bar{y}, x-y, 0 ; y-x, \bar{x}, 0 ; 00 \frac{1}{2}+(y x 0 ; x-y, \bar{y}, 0 ; \bar{x}, y-x, 0)$

$x y 0 ; \bar{y}, x-y, 0 ; y-x, \bar{x}, 0 ; 00 \frac{1}{2}+(\bar{x} \bar{y} 0 ; y, y-x, 0 ; x-y, x, 0)$

$\pm(x y 0) ; 00 \frac{1}{3} \pm(y-x, \bar{x}, 0) ; 00 \frac{2}{3} \pm(\bar{y}, x-y, 0)$

$\pm(x y 0 ; \bar{y}, x-y, 0 ; y-x, \bar{x}, 0) ; 00 \frac{1}{2} \pm(y x 0 ; x-y, \bar{y}, 0 ; \bar{x}, y-x, 0)$

$\left\{x y 0 ; \bar{y}, x-y, 0 ; y-x, \bar{x}, 0 ; 00 \frac{1}{2}+(\bar{x} \bar{y} 0 ; y-x, y, 0 ; x, x-y, 0)\right\} \tau$

$x, 0, \pm z ; 0, x, \pm z ; \bar{x}, \bar{x}, \pm z ; 00 \frac{1}{2}+(\bar{x}, 0, \pm z ; 0, \bar{x}, \pm z ; x, x, \pm z)$

$x y 0 ; \bar{y}, x-y, 0 ; y-x, \bar{x}, 0 ; \bar{y} \bar{x} 0 ; y-x, y, 0 ; x, x-y, 0$;

$00 \frac{1}{2}+(\bar{x} \bar{y} 0 ; y, y-x, 0 ; x-y, x, 0 ; y x 0 ; x-y, \bar{y}, 0 ; \bar{x}, y-x, 0)$

$x, \bar{x}, \pm z ; x, 2 x, \pm z ; 2 \bar{x}, \bar{x}, \pm z ; 00 \frac{1}{2}+(\bar{x}, x, \pm z ; \bar{x}, 2 \bar{x}, \pm z ;$

$2 x, x, \pm z)$

$x, y, \pm z ; \bar{y}, x-y, \pm z ; y-x, \bar{x}, \pm z$

$x y z ; \bar{y}, x-y, z ; y-x, \bar{x}, z ; \bar{y} \bar{x} \bar{z} ; y-x, y, \bar{z} ; x, x-y, \bar{z}$

$x y z ; \bar{y}, x-y, z ; y-x, \bar{x}, z ; y x \bar{z} ; x-y, \bar{y}, \bar{z} ; \bar{x}, y-x, \bar{z}$

$\pm(x y z ; \bar{y}, x-y, z ; y-x, \bar{x}, z)$

$x, y, \pm z ; \bar{y}, x-y, \pm z ; y-x, \bar{x}, \pm z ; \bar{y}, \bar{x}, \pm z ; y-x, y, \pm z ;$

$x, x-y, \pm z$

$(x, y, \pm z ; \bar{y}, x-y, \pm z ; y-x, \bar{x}, \pm z) \tau$

$x y z ; \bar{y}, x-y, z ; y-x, \bar{x}, z ; \bar{x} \bar{y} z ; y, y-x, z ; x-y, x, z$;

$y x \bar{z} ; x-y, \bar{y}, \bar{z} ; \bar{x}, y-x, \bar{z} ; \bar{y} \bar{x} \bar{z} ; y-x, y, \bar{z} ; x, x-y, \bar{z}$

$\pm(x, y, \pm z ; \bar{y}, x-y, \pm z ; y-x, \bar{x}, \pm z)$

$\pm(x y z ; \bar{y}, x-y, z ; y-x, \bar{x}, z) \tau$

$\pm(x y z ; \bar{y}, x-y, z ; y-x, \bar{x}, z ; y x \bar{z} ; x-y, \bar{y}, \bar{z} ; \bar{x}, y-x, \bar{z})$

$\pm(x, y, \pm z ; \bar{y}, x-y, \pm z ; y-x, \bar{x}, \pm z) \tau$

$x, y, \pm z ; \bar{y}, x-y, \pm z ; y-x, \bar{x}, \pm z ; 00 \frac{1}{2}+(\bar{y}, \bar{x}, \pm z ; y-x, y, \pm z ;$

$x, x-y, \pm z)$

$x, y, \pm z ; \bar{y}, x-y, \pm z ; y-x, \bar{x}, \pm z ; 00 \frac{1}{2}+(y, x, \pm z ; x-y, \bar{y}, \pm z ;$ $\bar{x}, y-x, \pm z)$

$x y z ; \bar{x} \bar{y} z ; x-y, \bar{y}, \bar{z} ; y-x, y, \bar{z} ; 00 \frac{1}{3}+(y-x, \bar{x}, z ; x-y, x, z$ $\bar{x}, y-x, \bar{z} ; x, x-y, \bar{z}) ; 00 \frac{2}{3}+(\bar{y}, x-y, z ; y, y-x, z ; y x \bar{z}, \bar{y} \bar{x} \bar{z})$ $x y z ; \bar{y}, x-y, z ; y-x, \bar{x}, z ; y x \bar{z} ; x-y, \bar{y}, \bar{z} ; \bar{x}, y-x, \bar{z} ;$

$00 \frac{1}{2}+(\bar{x} \bar{y} z ; y, y-x, z ; x-y, x, z ; \bar{y} \bar{x} \bar{z} ; y-x, y, \bar{z} ; x, x-y, \bar{z})$

$x, y, \pm z ; y, x-y, \pm z ; y-x, \bar{x}, \pm z ; 00 \frac{1}{2}+(\bar{x}, \bar{y}, \pm z ; \bar{y}, y-x, \pm z$;

$x-y, x, \pm z)$

$x y z ; \bar{y}, x-y, z ; y-x, \bar{x}, z ; \bar{y} \bar{x} \bar{z} ; y-x, y, \bar{z} ; x, x-y, \bar{z}$;

$00 \frac{1}{2}+(y x z ; x-y, \bar{y}, z ; \bar{x}, y-x, z ; \bar{x} \bar{y} \bar{z} ; y, y-x, \bar{z} ; x-y, x, \bar{z})$

$x y z ; \bar{y}, x-y, z ; y-x, \bar{x}, z ; y x \bar{z} ; x-y, \bar{y}, \bar{z} ; \bar{x}, y-x, \bar{z} ;$

$00 \frac{1}{2}+(\bar{y} \bar{x} z ; y-x, y, z ; x, x-y, z ; \bar{x} \bar{y} \bar{z}, y, y-x, \bar{z} ; x-y, x, \bar{z})$

$x y z ; \bar{y}, x-y, z ; y-x, \bar{x}, z ; \bar{x} \bar{y} z ; y, y-x, z ; x-y, x, z ; y x \bar{z}$;

$x-y, \bar{y}, \bar{z} ; \bar{x}, y-x, \bar{z} ; \bar{y} \bar{x} \bar{z} ; y-x, y, \bar{z} ; x, x-y, \bar{z} ; 00 \frac{1}{2}+(x y \bar{z} ;$

$\bar{y}, x-y, \bar{z} ; y-x, \bar{x}, \bar{z} ; \bar{x} \bar{y} \bar{z} ; y, y-x, \bar{z} ; x-y, \bar{x}, \bar{z} ; y x z ; x-y, \bar{y}, z$;

$\bar{x}, y-x, z ; \bar{y} \bar{x} z ; y-x, y, z ; x, x-y, z)$

$(x, y, \pm z ; \bar{y}, x-y, \pm z ; y-x, \bar{x}, \pm z) \tau ; 00 \frac{1}{2}+(\bar{x}, \bar{y}, \pm z ; y, y-x, \pm z ;$ $x-y, x, \pm z) \tau$

$x, y, \pm z ; \bar{y}, x-y, \pm z ; y-x, \bar{x}, \pm z ; \bar{y}, \bar{x}, \pm z ; y-x, y, \pm z ; x, x-y, \pm z ;$

$00 \frac{1}{2}+(y, x, \pm z ; x-y, \bar{y}, \pm z ; \bar{x}, y-x, \pm z ; \bar{x}, \bar{y}, \pm z ; y, y-x, \pm z$;

$x-y, x, \pm z)$

\begin{tabular}{|llll|}
\hline$G$ & $P 6 / \mathrm{mmm}$ & $2(c) \overline{6} m 2$ & $\pm\left(\frac{1}{3} \frac{2}{3} 0\right)$ \\
\hline
\end{tabular}

G2z

$P 6 / \operatorname{mmm}(h) \quad \pm\left(\frac{1}{3}, \frac{2}{3}, \pm z\right)$ 


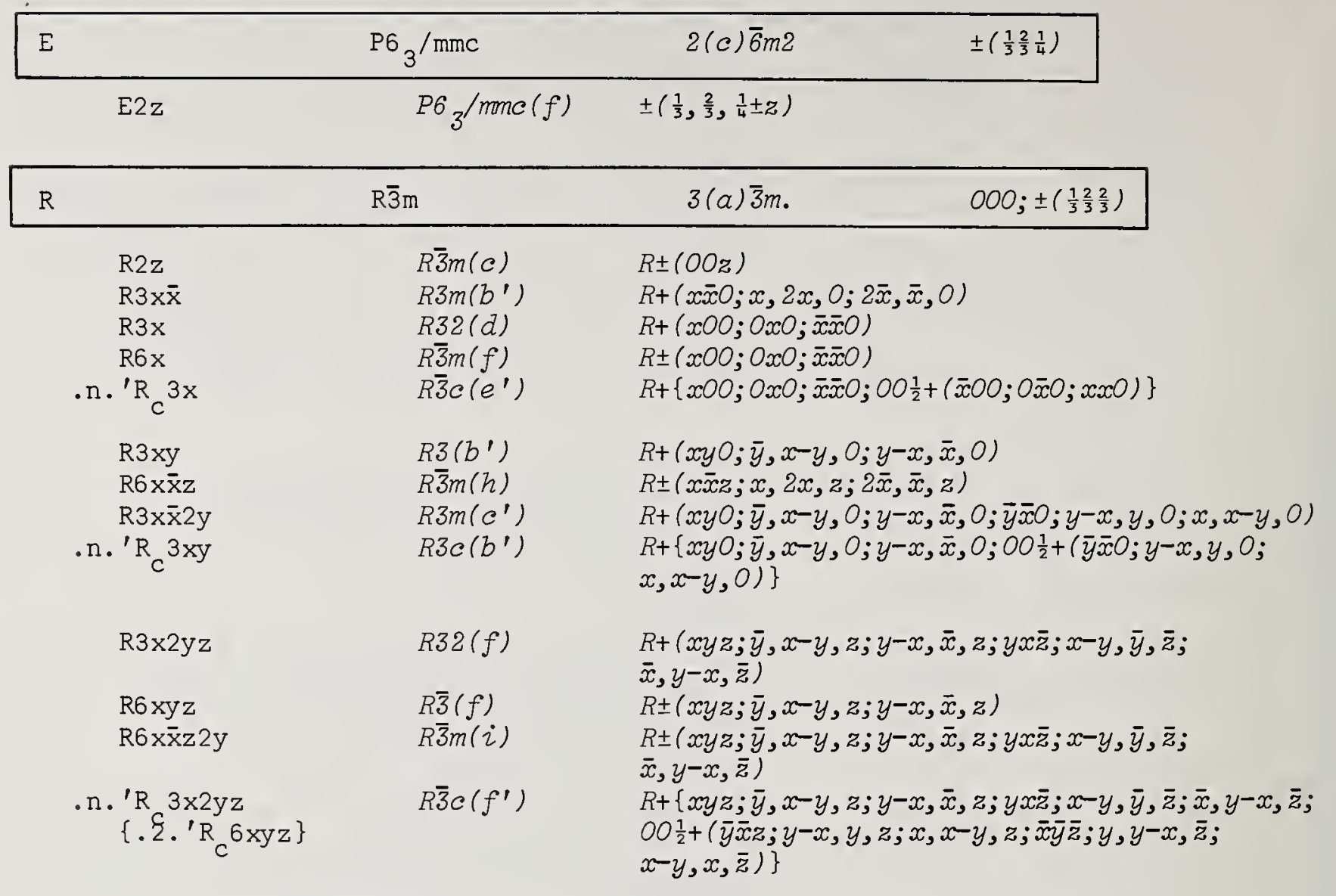

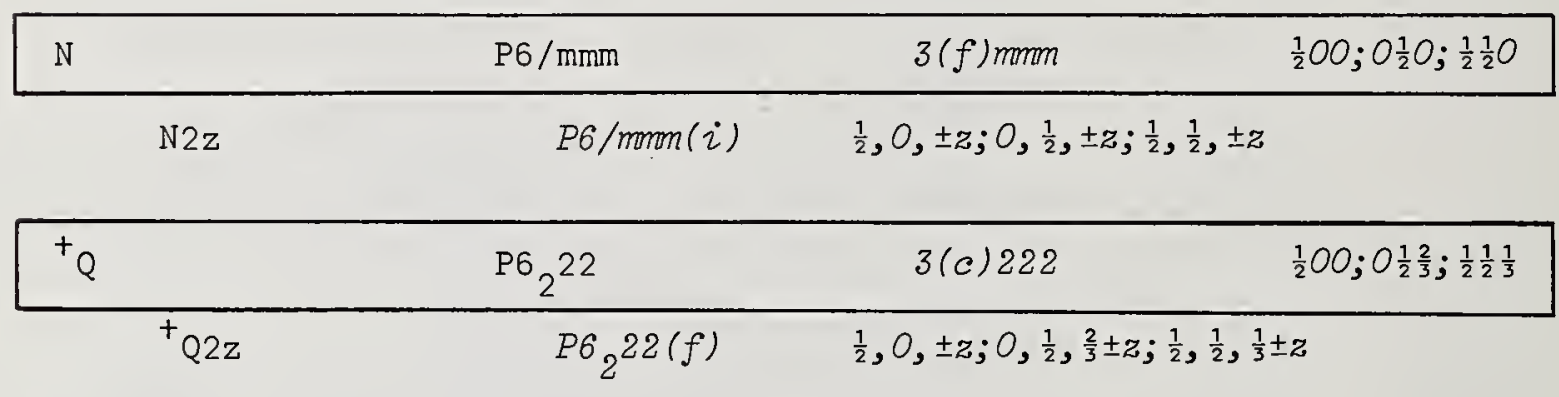

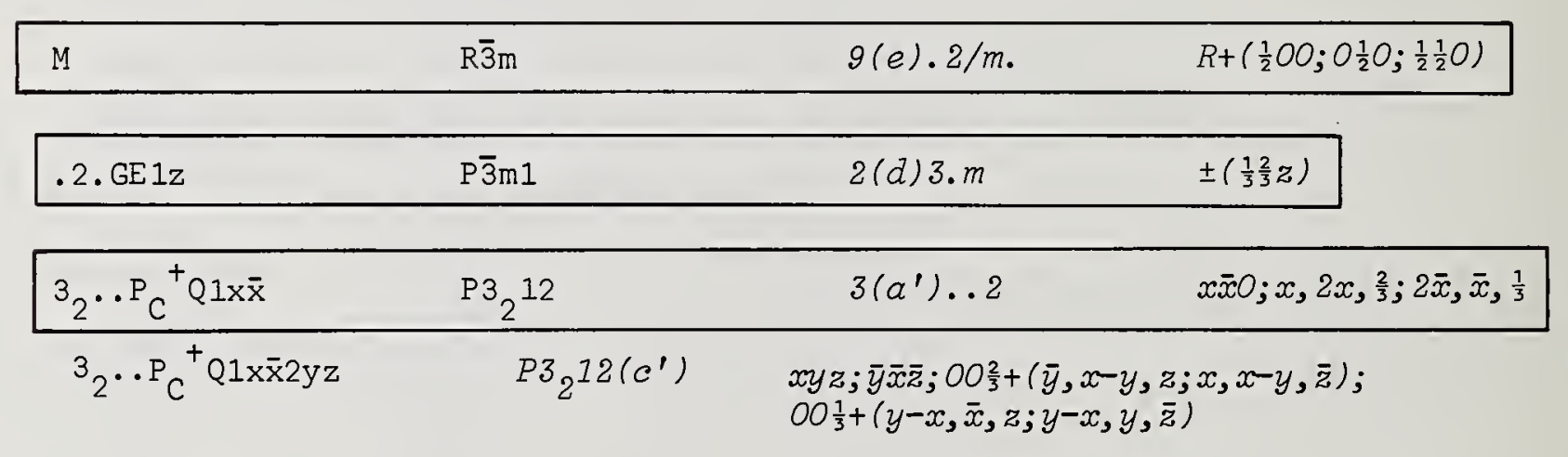

\begin{tabular}{|c|c|c|c|}
\hline${ }_{2}{ }_{2} \cdot P_{C}^{+} Q 1 x$ & $\mathrm{P}_{2} 21$ & $3\left(a^{\prime}\right) .2$ & $x 00 ; 0 x^{\frac{2}{3}} ; \bar{x} \bar{x} \frac{1}{3}$ \\
\hline${ }^{3}{ }_{2} \cdots P_{C}^{+} Q 1 x 2 y z$ & ${ }^{P 3}{ }_{2} 21\left(c^{\prime}\right)$ & $\begin{array}{l}x y z ; x-y, \bar{y} ; \bar{z} \\
00 \frac{1}{3}+(y-x, \bar{x}\end{array}$ & $-y, z ; y x \bar{z}) ;$ \\
\hline
\end{tabular}




\begin{tabular}{|c|c|c|c|}
\hline${ }^{3} \mathrm{I}^{2 \mathrm{P}} \mathrm{Cc}{ }^{+} \mathrm{Q}_{\mathrm{C}} 1 \mathrm{x}$ & $\mathrm{P}_{1} 22$ & $6(a) .2$ & $x O O ; x x \frac{1}{6} ; O x \frac{1}{3} ; \bar{x} O \frac{1}{2} ; \bar{x} \bar{x}_{\frac{2}{3}} ; O \bar{x} \frac{5}{6}$ \\
\hline${ }^{3} \cdot 2 \mathrm{P} \mathrm{C}_{1}{ }^{+}{ }^{+} \cdot \mathrm{P}_{\mathrm{C}}{ }^{1}$ & $z^{P 6_{1} 22(c)}$ & $\begin{array}{l}x y z ; x-y, \bar{y}, \bar{z} \\
(\bar{y}, x-y, z ; y x \bar{z} \\
\bar{x}, y-x, \bar{z}) ; 00 \overline{\bar{c}}\end{array}$ & $\begin{array}{l}x-y, x, z ; x, x-y, \bar{z}) ; 00 \frac{1}{3}+ \\
+(\bar{x} \bar{y} z ; y-x, y, \bar{z}) ; 00 \frac{2}{3}+(y-x, \bar{x} \\
-x, z ; \bar{y} \bar{x} \bar{z})\end{array}$ \\
\hline
\end{tabular}

\begin{tabular}{|lll}
\hline${ }_{1} 2 . \mathrm{P}_{\mathrm{CC}{ }_{\mathrm{C}}{ }^{+} \mathrm{Q}_{\mathrm{C}} 1 \mathrm{x} \overline{\mathrm{x}}} \quad \mathrm{P}_{1}{ }_{1} 22$ & $6\left(\mathrm{~b}^{\prime}\right) . .2$ & \\
& & $\begin{array}{l}x \bar{x} 0 ; 2 x, x, \frac{1}{6} ; x, 2 x, \frac{1}{3} ; \bar{x} x \frac{1}{2} ; \\
2 \bar{x}, \bar{x}, \frac{2}{3} ; \bar{x}, 2 \bar{x}, \frac{5}{6}\end{array}$ \\
\hline
\end{tabular}

\begin{tabular}{|c|c|c|c|}
\hline${ }^{3}{ }_{2} \cdots \mathrm{P}_{C}^{+} \mathrm{Q} 1 \mathrm{xy}$ & $\mathrm{P}_{2}$ & $3\left(\alpha^{\prime}\right) 1$ & $x y 0 ; \bar{y}, x-y, \frac{2}{3} ; y-x, \bar{x}, \frac{1}{3}$ \\
\hline${ }^{3}{ }_{1}{ }_{1} \ldots P_{C c} E_{C}^{+} Q_{C} 1 x y$ & $\mathrm{Po}_{1}$ & $6\left(\alpha^{\prime}\right) 1$ & $\begin{array}{l}x y 0 ; x-y, x, \frac{1}{6} ; \bar{y}, x-y, \frac{1}{3} ; \bar{x} \bar{y} \frac{1}{2} ; \\
y-x, \bar{x}, \frac{2}{3} ; y, y-x, \frac{5}{6}\end{array}$ \\
\hline
\end{tabular}

Table 20: Rhombohedral complexes in rhombohedral description

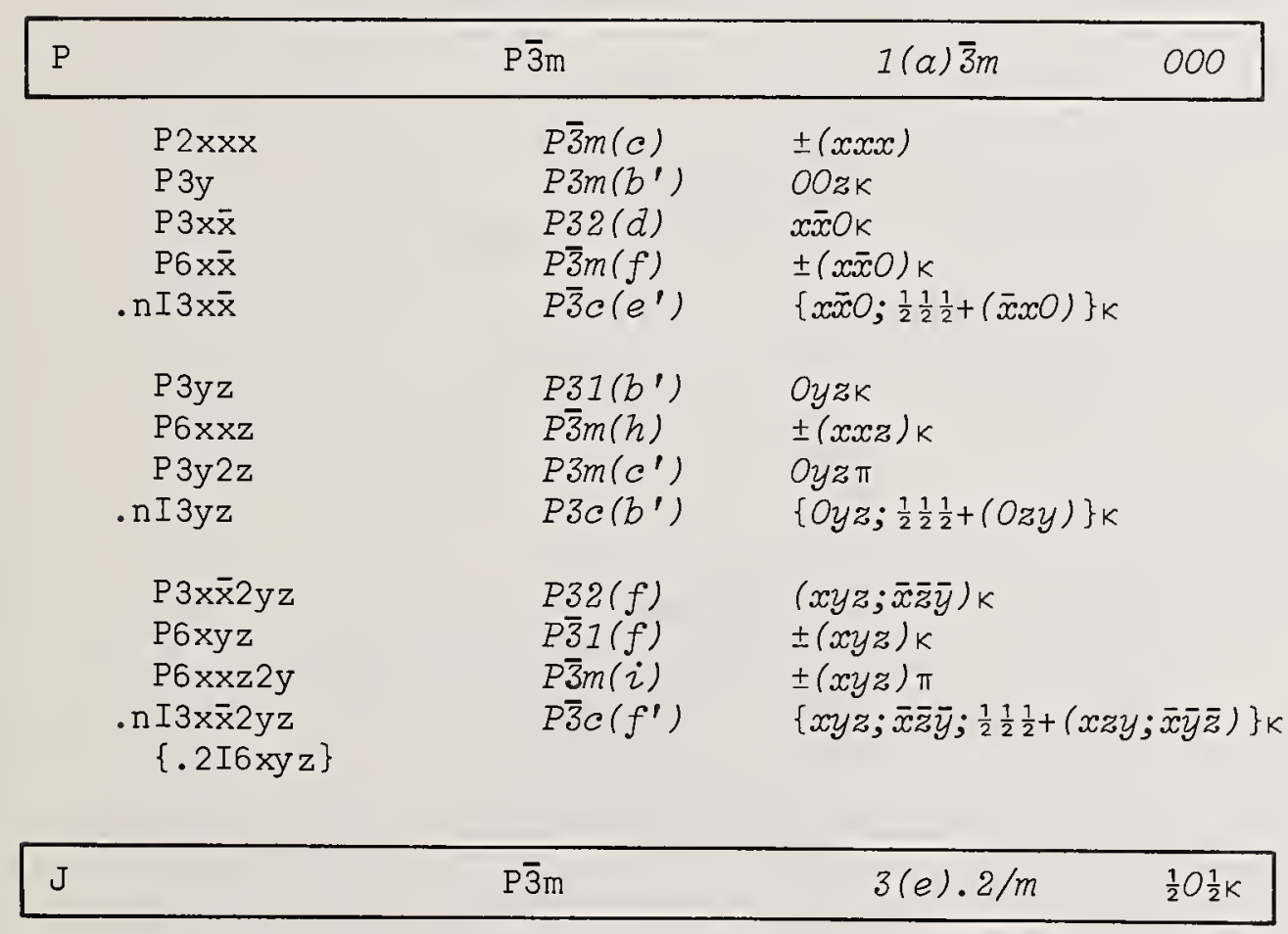


Table 21: Tetragonal System

\begin{tabular}{|c|c|c|}
\hline $\mathrm{P} 4 / \mathrm{mmm}$ & & $1(a) 4 / m m m$ \\
\hline $\mathrm{P} 2 \mathrm{z}$ & $P 4 /$ mron $(g)$ & $\pm(00 z)$ \\
\hline $\mathrm{P} 4 \mathrm{xx}$ & $P 4 / \operatorname{mrn}(j)$ & $\pm(x, \pm x, 0)$ \\
\hline $\mathrm{P} 4 \mathrm{x}$ & $P 4 / m m m(Z)$ & $\pm(x 00) \tau$ \\
\hline$\cdot \mathrm{b} \cdot \mathrm{c} 2 \mathrm{xx}$ & $\mathrm{P} 4 / \mathrm{mbm}\left(g^{\prime}\right)$ & $\pm(x x 0) ; \frac{1}{2} \frac{1}{2} O \pm(x \bar{x} 0)$ \\
\hline$\ldots 2 \mathrm{P} I 2 \mathrm{x}$ & $P 4_{2} / m m c(j)$ & $\pm\left(x 00 ; 0 x \frac{1}{2}\right)$ \\
\hline .2. $P^{C} 2 x x$ & $P 4 / \mathrm{mom}(i)$ & $\pm\left(x x 0 ; x \bar{x} \frac{1}{2}\right)$ \\
\hline$\cdot b \cdot c_{c}^{c} 2 x x$ & $\operatorname{I4}{ }^{\prime} \mathrm{mem}\left(h^{\prime}\right)$ & $I \pm\left(x x 0 ; x \bar{x} \frac{1}{2}\right)$ \\
\hline P4xxz & $P \overline{4} 2 m(n)$ & $x x z ; \bar{x} \bar{x} z ; \bar{x} x \bar{z} \tau$ \\
\hline $\mathrm{P} 4 \mathrm{xz}$ & $P \overline{4} m 2(j)$ & $\pm x, 0, z ; 0, \pm x, \bar{z}$ \\
\hline P4xy & $P 4 / m(j)$ & $\pm(x y 0 ; \bar{y} x 0)$ \\
\hline $\mathrm{P} 4 \mathrm{x} 2 \mathrm{y}$ & $P 4 /$ moron $(p)$ & $\pm(x y 0 ; \bar{y} x 0) \tau$ \\
\hline $\mathrm{P} 4 \times \times 2 \mathrm{z}$ & $P 4 / \operatorname{mman}(r)$ & $\pm(x, x, \pm z ; x, \bar{x}, \pm z)$ \\
\hline $\mathrm{P} 4 \times 2 \mathrm{z}$ & $P 4 /$ mron $(s)$ & $\pm(x, 0, \pm z) \tau$ \\
\hline$\overline{4}^{-1} \ldots \mathrm{P}$ I2xy & $P 4_{2} / m(j)$ & $\pm\left(x y 0 ; \bar{y} x \frac{1}{2}\right)$ \\
\hline $.2 . P^{C} 4 x y$ & $P 44 m c c(m)$ & $\pm\left(x y 0 ; \bar{y} x 0 ; \bar{x} y \frac{1}{2} ; y x \frac{1}{2}\right)$ \\
\hline . 2C4 $\mathrm{xxz}$ & $P 4 / n b m\left(m^{\prime}\right)$ & $x x z ; \bar{x} \bar{x} z ; \bar{x} x \bar{z} \tau ; \frac{1}{2} \frac{1}{2} O+(x x \bar{z} ; \bar{x} \bar{x} \bar{z} ; \bar{x} x z \tau)$ \\
\hline $\begin{array}{l}\cdots m C 4 x y \\
\quad\{. b . c 2 x \times 2 y\}\end{array}$ & $P 4 / m b m(i)$ & $\pm(x y 0 ; \bar{y} x O) ; \frac{1}{2} \frac{1}{2} O \pm(\bar{x} y 0 ; y x O)$ \\
\hline .b. $22 \times \times 2 z$ & $P 4 / m b m\left(k^{\prime}\right)$ & $\pm(x, x, \pm z) ; \frac{1}{2} \frac{1}{2} O \pm(\bar{x}, x, \pm z)$ \\
\hline $\begin{array}{l}\ldots m C 4 x z \\
\quad\{.2 C I 1 z 4 x\}\end{array}$ & $P 4 /$ nmm $(i)$ & $\pm x, 0, z ; 0, \pm x, \bar{z} ; \frac{1}{2} \frac{1}{2} O+(0, \pm x, z ; \pm x, 0, \bar{z})$ \\
\hline $\begin{array}{l}\text { m.. } \\
{ }_{\{} c^{4 \times z} \\
\cdots 2 \mathrm{P} \text { I } 2 \times 2 z\}\end{array}$ & $P 4_{2} / m m c\left(O^{\prime}\right)$ & $\pm x, 0, z ; 0, \pm x, \bar{z} ; 00 \frac{1}{2}+( \pm x, 0, \bar{z} ; 0, \pm x, z)$ \\
\hline$\ldots 2 \mathrm{P} I 2 \mathrm{I} 2 \mathrm{y}$ & $P 42^{2} /$ mme (q) & $\pm\left(x y 0 ; \bar{x} y 0 ; y x \frac{1}{2} ; y \bar{x} \frac{1}{2}\right)$ \\
\hline $\begin{array}{l}\cdot . P c_{4 \times x z} \\
\left\{c^{2} \cdot P 2 \times x 2 z\right\}\end{array}$ & ${ } 4_{2} / \operatorname{mem}\left(0^{\prime}\right)$ & $x x z ; \bar{x} \bar{x} z ; \bar{x} x \bar{z} \tau ; 00 \frac{1}{2}+(x x \bar{z} ; \bar{x} \bar{x} \bar{z} ; \bar{x} x z \tau)$ \\
\hline. $\mathrm{b} 2 \mathrm{C} 2 \mathrm{xy} \mathrm{c}^{2}$ & $P 42 / m b c(h)$ & $\pm(x y 0) ; \frac{1}{2} \frac{1}{2} O \pm(\bar{x} y 0) ; 00 \frac{1}{2} \pm(y \bar{x} 0) ; \frac{1}{2} \frac{1}{2} \frac{1}{2} \pm(y x 0)$ \\
\hline 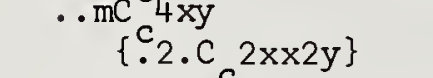 & $I 4 / \mathrm{mem}(k)$ & $I \pm\left(x y 0 ; y \bar{x} 0 ; \bar{x} y \frac{1}{2} ; y x \frac{1}{2}\right)$ \\
\hline $\begin{array}{l}. \text { b.c } 4 \times x z \\
\left\{\text { c.b.c } c^{2 \times x 2 z}\right\}\end{array}$ & $I 4 / \mathrm{mem}\left(乙^{\prime}\right)$ & $I+\left\{x x z ; \bar{x} \bar{x} z ; \bar{x} x \bar{z} \tau ; \frac{1}{2} \frac{1}{2} O+(\bar{x} x z \tau ; x x \bar{z} ; \bar{x} \bar{x} \bar{z})\right\}$ \\
\hline $\mathrm{P} 4 x y z$ & $P \overline{4}(h)$ & $x y z ; \bar{x} \bar{y} z ; y \bar{x} \bar{z} ; \bar{y} x \bar{z}$ \\
\hline $\mathrm{P} 4 \mathrm{xxz} 2 \mathrm{y}$ & $P \overline{4} 2 m(0)$ & $(x y z ; \bar{x} \bar{y} z ; y \bar{x} \bar{z} ; \bar{y} x \bar{z}) \tau$ \\
\hline $\mathrm{P} 4 x z 2 y$ & $P \overline{4} m 2($ (Z) & $x, \pm y, z ; \bar{x}, \pm y, z ; y, \pm x, \bar{z} ; \bar{y}, \pm x, \bar{z}$ \\
\hline $\mathrm{P} 4 \mathrm{x} 2 \mathrm{yz}$ & $P 422(p)$ & $x y z ; \bar{x} \bar{y} z ; \bar{x} y \bar{z} ; x \bar{y} \bar{z} ; \bar{y} \bar{x} \bar{z} ; y x \bar{z} ; y \bar{x} z ; \bar{y} x z$ \\
\hline P4xy $2 z$ & $P 4 / m(Z)$ & $\pm(x, y, \pm z ; \bar{y}, x, \pm z)$ \\
\hline P $4 \times 2 y 2 z$ & $P 4 / m m m(u)$ & $\pm(x, y, \pm z ; \bar{y}, x, \pm z) \tau$ \\
\hline $\begin{array}{l}\text { 2. }{ }^{P^{4}}{ }^{4} x y z \\
4\end{array}$ & $P \overline{4} 2 c(n)$ & $x y z ; \bar{x} \bar{y} z ; y \bar{x} \bar{z} ; \bar{y} x \bar{z} ; 00 \frac{1}{2}+(\bar{x} y \bar{z} ; x \bar{y} \bar{z} ; y x z ; \bar{y} \bar{x} z)$ \\
\hline$\cdots \quad\left\{\begin{array}{l}m y z \\
\quad 21 . C I 1 z 2 x \times 2 y\}\end{array}\right.$ & $\bar{P} \overline{4}_{1} m(f)$ & $x y z ; \bar{x} \bar{y} z ; y \bar{x} \bar{z} ; \bar{y} x \bar{z} ; \frac{1}{2} \frac{1}{2} O+(y x z ; \bar{y} \bar{x} z ; \bar{x} y \bar{z} ; x \bar{y} \bar{z})$ \\
\hline 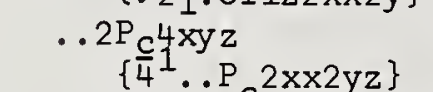 & $P \Psi c 2(j)$ & $x y z ; \bar{x} \bar{y} z ; y \bar{x} \bar{z} ; \bar{y} x \bar{z} ; 00 \frac{1}{2}+(y x \bar{z} ; \bar{y} \bar{x} \bar{z} ; \bar{x} y z ; x \bar{y} z)$ \\
\hline $\begin{array}{c}\ldots 2 \mathrm{C} 4 \mathrm{xyz} \mathrm{c}^{2 \mathrm{x} x \mathrm{y}<\mathrm{J}} \\
\{. \mathrm{b} \cdot \mathrm{C} 2 \mathrm{x} \times 2 \mathrm{yz}\}\end{array}$ & $P 4 b 2(i)$ & $x y z ; \bar{x} \bar{y} z ; y \bar{x} \bar{z} ; \bar{y} x \bar{z} ; \frac{1}{2} \frac{1}{2} O+(y x \bar{z} ; \bar{y} \bar{x} \bar{z} ; \bar{x} y z ; x \bar{y} z)$ \\
\hline 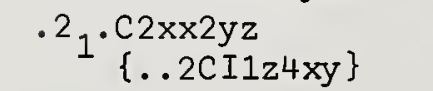 & $P 42_{1} 2(g)$ & $x y z ; \bar{x} \bar{y} z ; y x \bar{z} ; \bar{y} \bar{x} \bar{z} ; \frac{1}{2} \frac{1}{2} O+(\bar{x} y \bar{z} ; x \bar{y} \bar{z} ; \bar{y} x z ; y \bar{x} z)$ \\
\hline
\end{tabular}




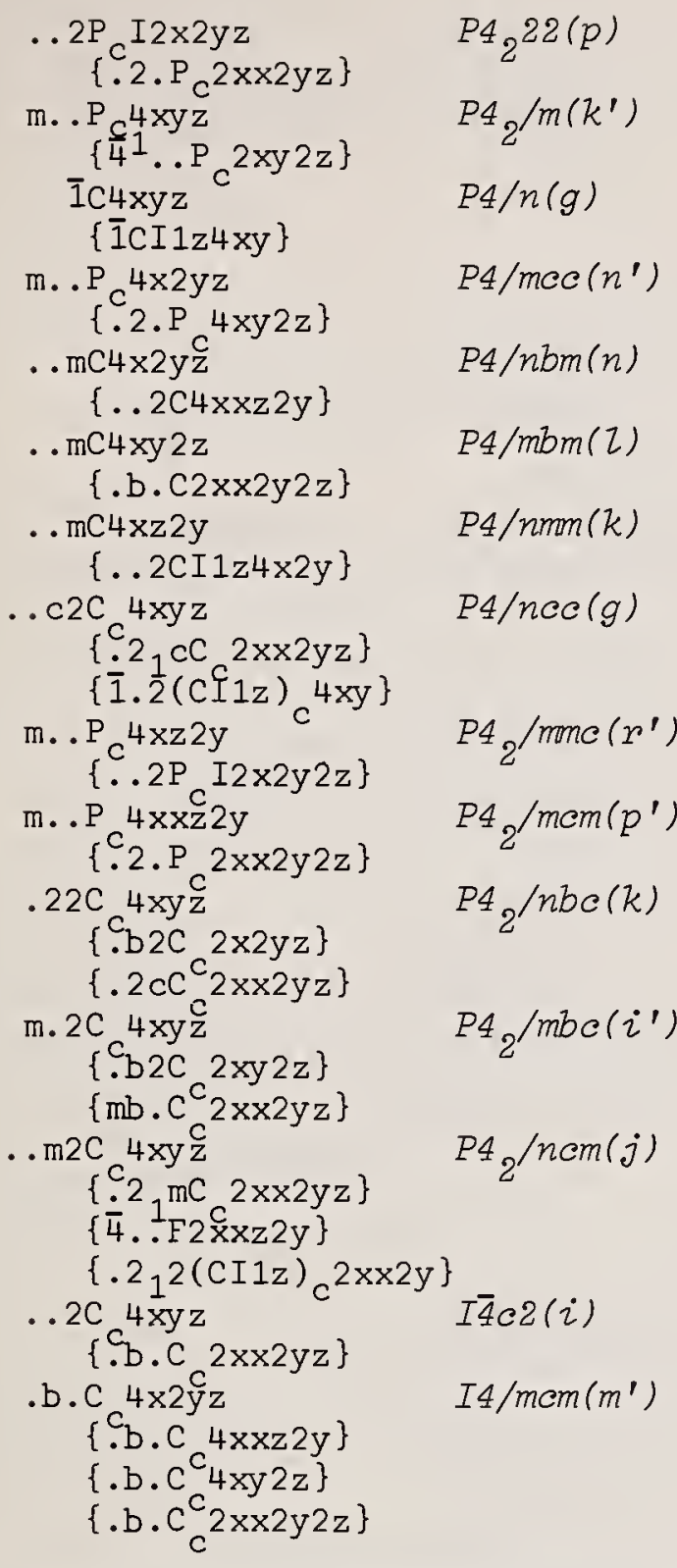

$x y z ; \bar{x} \bar{y} z ; \bar{x} y \bar{z} ; x \bar{y} \bar{z} ; 00 \frac{1}{2}+(y x \bar{z} ; \bar{y} \bar{x} z ; y \bar{x} z ; \bar{y} x z)$

$x y z ; \bar{x} \bar{y} z ; y \bar{x} \bar{z} ; \bar{y} x \bar{z} ; 00 \frac{1}{2}+(x y \bar{z} ; \bar{x} \bar{y} \bar{z} ; y \bar{x} z ; \bar{y} x z)$

$x y z ; \bar{x} \bar{y} z ; y \bar{x} \bar{z} ; \bar{y} x \bar{z} ; \frac{1}{2} \frac{1}{2} O+(\bar{x} \bar{y} \bar{z} ; x y \bar{z} ; \bar{y} x z ; y \bar{x} z)$

$x y z ; \bar{x} \bar{y} z ; \bar{x} y \bar{z} ; x \bar{y} \bar{z} ; \bar{y} \bar{x} \bar{z} ; y x \bar{z} ; y \bar{x} z ; \bar{y} x z ;$ $00 \frac{1}{2}+(x y \bar{z} ; \bar{x} \bar{y} \bar{z} ; \bar{x} y z ; x \bar{y} z ; \bar{y} \bar{x} z ; y x z ; y \bar{x} \bar{z} ; \bar{y} x \bar{z})$

$x y z ; \bar{x} \bar{y} z ; \bar{x} y \bar{z} ; x \bar{y} \bar{z} ; \bar{y} \bar{x} \bar{z} ; y x \bar{z} ; y \bar{x} z ; \bar{y} x z ;$

$\frac{1}{2} \frac{1}{2} O+(y x z ; \bar{y} \bar{x} z ; y \bar{x} \bar{z} ; \bar{y} x \bar{z} ; \bar{x} \bar{y} \bar{z} ; x y \bar{z} ; \bar{x} y z ; x \bar{y} z)$

$\pm(x, y, \pm z ; \bar{y}, x, \pm z) ; \frac{1}{2} \frac{1}{2} O \pm(y, x, \pm z ; x, \bar{y}, \pm z)$

$x, \pm y, z ; \bar{x}, \pm y, z ; y, \pm x, \bar{z} ; \bar{y}, \pm x, \bar{z} ; \frac{1}{2} \frac{1}{2} 0+( \pm y, x, z$; $\pm y, \bar{x}, z ; \pm x, y, \bar{z} ; \pm x, \bar{y}, \bar{z})$

$x y z ; \bar{x} \bar{y} z ; y \bar{x} \bar{z} ; \bar{y} x \bar{z} ; \frac{1}{2} \frac{1}{2} O+(x y \bar{z} ; \bar{x} \bar{y} \bar{z} ; y \bar{x} z ; \bar{y} x z) ;$

$00 \frac{1}{2}+(y x \bar{z} ; \bar{y} \bar{x} \bar{z} ; \bar{x} y z ; x \bar{y} z) ; \frac{1}{2} \frac{1}{2} \frac{1}{2}+(y x z ; \bar{y} \bar{x} z ; \bar{x} y \bar{z} ; x \bar{y} \bar{z})$

$x, \pm y, z ; \bar{x}, \pm y, z ; y, \pm x, \bar{z} ; \bar{y}, \pm x, \bar{z} ; 00 \frac{1}{2}+(x, \pm y, \bar{z} ;$

$\bar{x}, \pm y, \bar{z} ; y, \pm x, z ; \bar{y}, \pm x, z)$

( $x y z ; \bar{x} \bar{y} z ; y \bar{x} \bar{z} ; \bar{y} x \bar{z}) \tau ; 00 \frac{1}{2}+(x y \bar{z} ; \bar{x} \bar{y} \bar{z} ; y \bar{x} z ; \bar{y} x z) \tau$

$x y z ; \bar{x} \bar{y} z ; y \bar{x} \bar{z} ; \bar{y} x \bar{z} ; \frac{1}{2} \frac{1}{2} O+(y x \bar{z} ; \bar{y} \bar{x} \bar{z} ; \bar{x} y z ; x \bar{y} z)$;

$00 \frac{1}{2}+(\bar{x} y \bar{z} ; x \bar{y} \bar{z} ; \bar{y} \bar{x} z ; y x z) ; \frac{1}{2} \frac{1}{2} \frac{1}{2}+(y \bar{x} z ; \bar{y} x z ; \bar{x} \bar{y} \bar{z} ; x y \bar{z})$

$x y z ; \bar{x} \bar{y} z ; y \bar{x} \bar{z} ; \bar{y} x \bar{z} ; \frac{1}{2} \frac{1}{2} O+(y x \bar{z} ; \bar{y} \bar{x} \bar{z} ; \bar{x} y z ; x \bar{y} z) ;$

$00 \frac{1}{2}+(x y \bar{z} ; \bar{x} \bar{y} \bar{z} ; y \bar{x} z ; \bar{y} x z) ; \frac{1}{2} \frac{1}{2} \frac{1}{2}+(y x z ; \bar{y} \bar{x} z ; \bar{x} y \bar{z} ; x \bar{y} \bar{z})$

$x y z ; \bar{x} \bar{y} z ; y \bar{x} \bar{z} ; \bar{y} x \bar{z} ; \frac{1}{2} \frac{1}{2} O+(y x z ; \bar{y} \bar{x} z ; \bar{x} y \bar{z} ; x \bar{y} \bar{z})$;

$00 \frac{1}{2}+(y x \bar{z} ; \bar{y} \bar{x} \bar{z} ; \bar{x} y z ; x \bar{y} z) ; \frac{1}{2} \frac{1}{2} \frac{1}{2}+(x y \bar{z} ; \bar{x} \bar{y} \bar{z} ; y \bar{x} z ; \bar{y} x z)$

$I+\left\{x y z ; \bar{x} \bar{y} z ; y \bar{x} \bar{z} ; \bar{y} x \bar{z} ; \frac{1}{2} \frac{1}{2} O+(\bar{x} y z ; x \bar{y} z ; \bar{y} \bar{x} \bar{z} ; y x \bar{z})\right\}$

$I+\{x y z ; \bar{x} \bar{y} z ; \bar{x} y \bar{z} ; x \bar{y} \bar{z} ; \bar{y} \bar{x} \bar{z} ; y x \bar{z} ; y \bar{x} z ; \bar{y} x z ;$

$\left.O O \frac{1}{2}+(x y \bar{z} ; \bar{x} \bar{y} \bar{z} ; \bar{x} y z ; x \bar{y} z ; \bar{y} \bar{x} z ; y x z ; y \bar{x} \bar{z} ; \bar{y} x \bar{z})\right\}$

\begin{tabular}{|c|c|c|}
\hline I & $2(a) 4 / \mathrm{mmm}$ & $000 ; \frac{1}{2} \frac{1}{2} \frac{1}{2}$ \\
\hline I2z & $I 4 / m m m(e)$ & $I \pm(00 z)$ \\
\hline$I 4 x x$ & $I 4 / m m m(h)$ & $I \pm(x, \pm x, 0)$ \\
\hline$I 4 x$ & $I 4 / m m m(i)$ & $I \pm(x 00) \tau$ \\
\hline . n. I $2 x x$ & $P 4_{2} / \mathrm{mnm}(f)$ & $\pm(x x 0) ; \frac{1}{2} \frac{1}{2} \frac{1}{2} \pm(x \bar{x} 0)$ \\
\hline $.2 . F c^{2 x x}$ & $I 4_{1}^{2} / \operatorname{acd}\left(f^{\prime}\right)$ & $I+\left\{ \pm(x x O) ; \frac{1}{2} \frac{1}{2} O \pm(x \bar{x} O) ; \frac{1}{2} O \frac{1}{4} \pm(x x O) ; O \frac{1}{2} \frac{1}{4} \pm(x \bar{x} O)\right\}$ \\
\hline $14 x z$ & $I \overline{4} m 2(i)$ & $I+( \pm x, 0, z ; 0, \pm x, \bar{z})$ \\
\hline$I 4 x \times z$ & $I \overline{4} 2 m(i)$ & $I+(x x z ; \bar{x} \bar{x} z ; \bar{x} x \bar{z} \tau)$ \\
\hline I4xy & $I 4 / m(h)$ & $I \pm(x y 0 ; \bar{y} x 0)$ \\
\hline$I 4 x 2 y$ & $I 4 / \operatorname{mmon}(Z)$ & $I \pm(x y 0 ; \bar{y} x 0) \tau$ \\
\hline$I 4 \times x 2 z$ & $I 4 / m m m(m)$ & $I \pm(x, x, \pm z ; x, \bar{x}, \pm z)$ \\
\hline $\mathrm{I} 4 \mathrm{x} 2 \mathrm{z}$ & $I 4 / \operatorname{mmm}(n)$ & $I \pm(x, 0, \pm z) \tau$ \\
\hline ..2 2 4 xy & $P 4 / m n c(h)$ & $\pm(x y 0 ; \bar{y} x 0) ; \frac{1}{2} \frac{1}{2} \frac{1}{2} \pm(y x 0 ; x \bar{y} 0)$ \\
\hline. $.2 \mathrm{I} 4 \mathrm{xxz}$ & $P 4{ }_{2} / \mathrm{nnm}(\mathrm{m})$ & $x x z ; \bar{x} \bar{x} z ; \bar{x} x \bar{z} \tau ; \frac{1}{2} \frac{1}{2} \frac{1}{2}+(x x \bar{z} ; \bar{x} \bar{x} \bar{z} ; x \bar{x} z \tau)$ \\
\hline .n. $12 x \times 2 y$ & $P 4_{2}^{2} / m n m(i)$ & $\pm(x y 0) \tau ; \frac{1}{2} \frac{1}{2} \frac{1}{2} \pm(x \bar{y} O) \tau$ \\
\hline
\end{tabular}




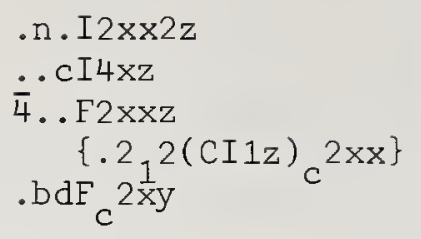

I4xyz

$14 \mathrm{xz} 2 \mathrm{y}$

$\mathrm{I} 4 \mathrm{xxz} 2 \mathrm{y}$

$14 x 2 y z$

$14 x y 2 z$

$14 \times 2 y 2 z$

..cI $4 x y z$

..2I $4 x y z$

$\{. \mathrm{n} . \mathrm{I} 2 \mathrm{x} x 2 \mathrm{yz}\}$

$.2, \cdot 12 \mathrm{xx} 2 \mathrm{yz}$

n.. $14 x y z$

..cI $4 x 2 y z$

..2I $4 x y 2 z$

..2I $4 x x z 2 y$

.n. I $2 x \times 2 y 2 z$

..cI $4 x z 2 y$

$.22 \mathrm{~F} 4 \mathrm{xyz}$

$\{. \mathrm{b} 2 \cdot \mathrm{F} 2 \mathrm{c} \times 2 \mathrm{yz}\}$
$P 4 \mathrm{mmm}(j) \quad \pm(x, x, \pm z) ; \frac{1}{2} \frac{1}{2} \frac{1}{2} \pm(x, \bar{x}, \pm z)$

$P 4_{2}^{2} / n m e(g)$

$P 4 \frac{2}{2} \operatorname{ncm}\left(i^{\prime}\right)$

$I 4_{1} c d\left(b^{\prime}\right)$

$I \overline{4}(g)$

$I \overline{4} m 2(j)$

$\operatorname{I} \overline{4} 2 m(j)$

$I 422(k)$

$I 4 / m(i)$

$I 4 / \operatorname{mmm}(0)$

$P \overline{4} 2$ c $(e)$

PĀnz $(i)$

$P 42^{2} 1^{2}(g)$
$P 4^{2} / n(g)$
$P 4^{2} n n c(k)$

P4/mne (i)

$P 4$ Innm(n)

$P 4 \frac{2}{m m m}(k)$

$P 4_{2}^{2} /$ nme (h)

I4 $1 / \operatorname{acd}(g)$

$I+(x y z ; \bar{x} \bar{y} z ; y \bar{x} \bar{z} ; \bar{y} x \bar{z})$

$I+(x y z ; \bar{x} \bar{y} z ; y \bar{x} \bar{z} ; \bar{y} x \bar{z}) \tau$

$I \pm(x, y, \pm z ; \bar{y}, x, \pm z)$

$I \pm(x, y, \pm z ; y, \bar{x}, \pm z) \tau$

$\bar{y}, \pm x, z ; x, \pm y, \bar{z} ; \bar{x}, \pm y, \bar{z})$ $\pm x, 0, z ; 0, \pm x, \bar{z} ; \frac{1}{2} \frac{1}{2} \frac{1}{2}+(0, \pm x, z ; \pm x, 0, \bar{z})$

$\pm(x x z) ; \frac{1}{2} \frac{1}{2} O \pm(\bar{x} \bar{x} z) ; \frac{1}{2} O \frac{1}{2} \pm(\bar{x} x \bar{z}) ; O \frac{1}{2} \frac{1}{2} \pm(x \bar{x} \bar{z})$

$I+\left\{ \pm(x y O) ; \frac{1}{2} \frac{1}{2} O \pm(\bar{x} y O) ; \frac{1}{2} O \frac{1}{4} \pm(y x O) ; O \frac{1}{2} \frac{1}{4} \pm(y \bar{x} O)\right\}$

$I+(x, \pm y, z ; \bar{x}, \pm y, z ; y, \pm x, \bar{z} ; \bar{y}, \pm x, \bar{z})$

I+ (xyz; $\bar{x} \bar{y} z ; \bar{x} y \bar{z} ; x \bar{y} \bar{z} ; \bar{y} \bar{x} \bar{z} ; y x \bar{z} ; y \bar{x} z ; \bar{y} x z)$

$x y z ; \bar{x} \bar{y} z ; y \bar{x} \bar{z} ; \bar{y} x \bar{z} ; \frac{1}{2} \frac{1}{2} \frac{1}{2}+(y x z ; \bar{y} \bar{x} z ; \bar{x} y \bar{z} ; x \bar{y} \bar{z})$

$x y z ; \bar{x} \bar{y} z ; y \bar{x} \bar{z} ; \bar{y} x \bar{z} ; \frac{1}{2} \frac{1}{2} \frac{1}{2}+(y x \bar{z} ; \bar{y} \bar{x} \bar{z} ; \bar{x} y z ; x \bar{y} z)$

$x y z ; \bar{x} \bar{y} z ; y x \bar{z} ; \bar{y} \bar{x} \bar{z} ; \frac{1}{2} \frac{1}{2} \frac{1}{2}+(x \bar{y} \bar{z} ; \bar{x} y \bar{z} ; y \bar{x} z ; \bar{y} x z)$

$x y z ; \bar{x} \bar{y} z ; y \bar{x} \bar{z} ; \bar{y} x \bar{z} ; \frac{1}{2} \frac{1}{2} \frac{1}{2}+(x y \bar{z} ; \bar{x} \bar{y} \bar{z} ; y \bar{x} z ; \bar{y} x z)$

$x y z ; \bar{x} \bar{y} z ; \bar{x} y \bar{z} ; x \bar{y} \bar{z} ; \bar{y} \bar{x} \bar{z} ; y x \bar{z} ; y \bar{x} z ; \bar{y} x z ;$

$\frac{1}{2} \frac{1}{2} \frac{1}{2}+(y x z ; \bar{y} \bar{x} z ; y \bar{x} \bar{z} ; \bar{y} x \bar{z} ; \bar{x} \bar{y} \bar{z} ; x y \bar{z} ; \bar{x} y z ; x \bar{y} z)$

$\pm(x, y, \pm z ; \bar{y}, x, \pm z) ; \frac{1}{2} \frac{1}{2} \frac{1}{2} \pm(y, x, \pm z ; x, \bar{y}, \pm z)$

( $x y z ; \bar{x} \bar{y} z ; y \bar{x} \bar{z} ; \bar{y} x \bar{z}) \tau ; \frac{1}{2} \frac{1}{2} \frac{1}{2}+(y x \bar{z} ; \bar{y} \bar{x} \bar{z} ; \bar{x} y z ; x \bar{y} z) \mathrm{\tau}$

$\pm(x, y, \pm z) \tau ; \frac{1}{2} \frac{1}{2} \frac{1}{2} \pm(x, \bar{y}, \pm z) \tau$

$x, \pm y, z ; \bar{x}, \pm y, z ; y, \pm x, \bar{z} ; \bar{y}, \pm x, \bar{z} ; \frac{1}{2} \frac{1}{2} \frac{1}{2}+(y, \pm x, z$;

$x y z ; \bar{x} \bar{y} z ; y \bar{x} \bar{z} ; \bar{y} x \bar{z} ; \frac{1}{2} \frac{1}{2} O+(y x \bar{z} ; \bar{y} \bar{x} \bar{z} ; \bar{x} y z ; x \bar{y} z)$;

$\frac{1}{2} O \frac{1}{4}+(x \bar{y} \bar{z} ; \bar{x} y \bar{z} ; y x z ; \bar{y} \bar{x} z) ; O \frac{1}{2} \frac{1}{4}+(\bar{y} x z ; y \bar{x} z ; x y \bar{z} ; \bar{x} \bar{y} \bar{z})$

\begin{tabular}{|cccc|}
\hline $\mathrm{D}$ & $\mathrm{F}_{1} / \mathrm{ddm}$ & $8(a) \overline{4} 2 m$ & $F+\left(000 ; \frac{1}{4} \frac{1}{4} \frac{1}{4}\right)$ \\
$\mathrm{V}_{\mathrm{D}}$ & $\mathrm{I}_{1} / \mathrm{amd}$ & $4(a) \overline{4} m 2$ & $I+\left(000 ; 0 \frac{1}{2} \frac{1}{4}\right)$ \\
\hline
\end{tabular}

\begin{tabular}{|c|c|c|}
\hline $\begin{array}{l}\mathrm{v} D 2 \mathrm{z} \\
\mathrm{v}_{\mathrm{D}} \mathrm{xxx} \\
\mathrm{d}_{\mathrm{v}}^{\mathrm{v}} \mathrm{D} 2 \mathrm{x} \\
\mathrm{v}_{\mathrm{D}} \mathrm{x} \mathrm{x}\end{array}$ & $\begin{array}{l}\text { I4 } 1 \text { /and }(e) \\
\text { I4 } / \text { amd }(g) \\
\text { I4 }{\text { md }\left(b^{\prime}\right)} \\
\text { I4 } 22(d)\end{array}$ & $\begin{array}{l}I+\left(0,0, \pm z ; 0, \frac{1}{2}, \frac{1}{4} \pm z\right) \\
I+\left\{ \pm(x, \pm x, 0) ; O \frac{1}{2} \frac{1}{4} \pm(x, \pm x, 0)\right\} \\
I+\left\{ \pm(x 00) ; 0 \frac{1}{4} \pm(0 x 0)\right\} \\
I+\left\{ \pm(x x 0) ; O \frac{1}{2} \frac{1}{4} \pm(x \bar{x} 0)\right\}\end{array}$ \\
\hline
\end{tabular}

$4 \frac{1}{1} \cdot{ }_{\mathrm{v}}^{\mathrm{v}} \mathrm{D} 2 \mathrm{xy}$

.2. $\mathrm{D} 4 \mathrm{xz}$

.. $\mathrm{d}^{\mathrm{v}} \mathrm{D} 2 \mathrm{x} 2 \mathrm{y}$

.2. ${ }^{\mathrm{v}} \mathrm{D} 4 \mathrm{xyz}$

$I 4\left(b^{\prime}\right)$

I4 1 / and $(h)$

$I+\left\{ \pm(x y 0) ; O \frac{1}{2} \frac{1}{4} \pm(\bar{y} x O)\right\}$

I4 ${ }_{1}^{m d\left(c^{\prime}\right)}$

$I+\left\{ \pm x, 0, z ; 0, \pm x, \bar{z} ; 0 \frac{1}{2} \frac{1}{4}+( \pm x, 0, \bar{z} ; 0, \pm x, z)\right\}$

$I+\left\{ \pm(x, \pm y, 0) ; 0 \frac{1}{2} \frac{1}{4} \pm( \pm y, x, 0)\right\}$

. 2. $\mathrm{v} D 2 \mathrm{xx} 2 \mathrm{yz}$

a.. $\mathrm{v}$ D4xyz

$I \overline{4} 2 \mathrm{~d}(e)$

$I 4122(\mathrm{~g})$
$I 41 / \mathrm{f})$

.2. $\mathrm{D} 4 \mathrm{xz} 2 \mathrm{y}$

I4 1 / amd $(i)$

$I+\left\{x y z ; \bar{x} \bar{y} z ; y \bar{x} \bar{z} ; \bar{y} x \bar{z} ; O \frac{1}{2} \frac{1}{4}+(x \bar{y} \bar{z} ; \bar{x} y \bar{z} ; \bar{y} \bar{x} z ; y x z)\right\}$

$I+\left\{x y z ; \bar{x} \bar{y} z ; y x \bar{z} ; \bar{y} \bar{x} \bar{z} ; 0 \frac{1}{2} \frac{1}{4}+(x \bar{y} \bar{z} ; \bar{x} y \bar{z} ; y \bar{x} z ; \bar{y} x z)\right\}$

$I+\left\{x y z ; \bar{x} \bar{y} z ; y \bar{x} \bar{z} ; \bar{y} x \bar{z} ; 0 \frac{1}{2} \frac{1}{4}+(x y \bar{z} ; \bar{x} \bar{y} \bar{z} ; y \bar{x} z ; \bar{y} x z)\right\}$

$I+\left\{x, \pm y, z ; \bar{x}, \pm y, z ; y, \pm x, \bar{z} ; \bar{y}, \pm x, \bar{z} ; O \frac{1}{2} \frac{1}{4}+(x, \pm y, \bar{z} ;\right.$

$\bar{x}, \pm y, \bar{z} ; y, \pm x, z ; \bar{y}, \pm x, z)\}$

\begin{tabular}{|llll|}
\hline $\mathrm{T}$ & $\mathrm{F}_{4} / \mathrm{ddm}$ & $16(\mathrm{c}) . .2 / m$ & $F+\left(\frac{1}{8} \frac{1}{8} \frac{1}{8} ; \frac{3}{8} \frac{1}{8} \frac{3}{8} \pi\right)$ \\
$\mathrm{v}_{\mathrm{T}}$ & $\mathrm{I}_{1} / \mathrm{amd}$ & $8(\mathrm{c}) .2 / \mathrm{m}$. & $I+\left(0 \frac{1}{4} \frac{1}{8} ; 0 \frac{3}{4} \frac{1}{8} ; \frac{1}{4} O \frac{7}{8} ; \frac{3}{4} \mathrm{O} \frac{7}{8}\right)$ \\
\hline
\end{tabular}

$\ldots 2^{\mathrm{v}} \mathrm{T} 2 \mathrm{x}$

$I_{1} /$ amd $(f)$

$I+\left( \pm x, \frac{1}{4}, \frac{1}{8} ; \pm x, \frac{3}{4}, \frac{1}{8} ; \frac{1}{4}, \pm x, \frac{7}{8} ; \frac{3}{4}, \pm x, \frac{7}{8}\right)$ 


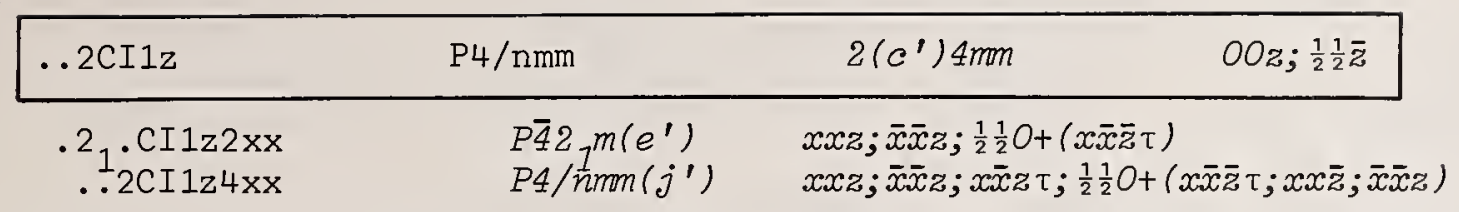

\begin{tabular}{|c|c|c|c|}
\hline${ }^{4} \cdot \mathrm{P}_{\mathrm{cc}} \mathrm{I}_{\mathrm{c}} 1 \mathrm{x}$ & $\mathrm{P}_{4} 3^{22}$ & $4\left(a^{\prime}\right) .2$ & $x O O ; O x \frac{3}{4} ; \bar{x} O \frac{1}{2} ; O \bar{x} \frac{1}{4}$ \\
\hline
\end{tabular}

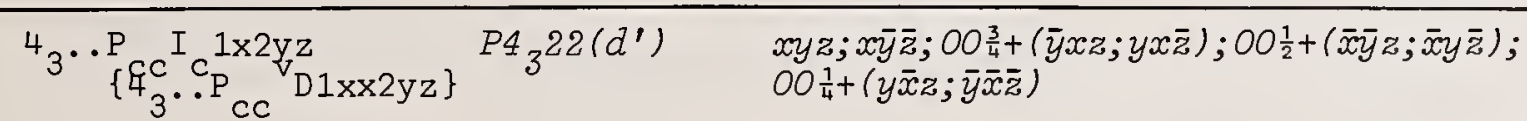

\begin{tabular}{|c|c|c|c|}
\hline${ }^{4} 3 \cdot P_{c c}{ }^{V_{D} 1 x x}$ & $\mathrm{P}_{3}{ }^{22}$ & $4\left(c^{\prime}\right) \ldots 2$ & $x x_{0} ; \bar{x} x \frac{3}{4} ; \bar{x} \bar{x} \frac{1}{2} ; x \bar{x} \frac{1}{4}$ \\
\hline${ }^{4}{ }_{3} \ldots I_{c}{ }^{V_{D} 1 x x}$ & $\mathrm{P}_{4} 3^{2} 1^{2}$ & $4(a) \ldots 2$ & $\begin{array}{l}x x 0 ; \frac{1}{2} \frac{1}{2} \frac{1}{4}+(x \bar{x} 0) ; \bar{x} \bar{x} \frac{1}{2} ; \\
\frac{1}{2} \frac{1}{2} \frac{3}{4}+(\bar{x} x 0)\end{array}$ \\
\hline
\end{tabular}

$\begin{array}{ll}{ }_{4} \ldots I_{c}{ }^{V_{D} 1 x x 2 y z} \quad P 43^{2} I^{2(b)} & x y z ; y x \bar{z} ; \frac{1}{2} \frac{1}{2} \frac{1}{4}+(y \bar{x} z ; x \bar{y} \bar{z}) ; 00 \frac{1}{2}+(\bar{x} \bar{y} z ; \bar{y} \bar{x} \bar{z}) ; \\ & \frac{1}{2} \frac{1}{2} \frac{3}{4}+(\bar{y} x z ; \bar{x} y \bar{z})\end{array}$

\begin{tabular}{|llll|}
\hline$\overline{4} . \mathrm{V}_{\mathrm{TF}}{ }_{\mathrm{C}} 1 \mathrm{x}$ & $\mathrm{I} \overline{4} 2 \mathrm{~d}$ & $8(d) .2$. & $I+\left(x \frac{1}{4} \frac{1}{8} ; \bar{x} \frac{3}{4} \frac{1}{8} ; \frac{3}{4} x \frac{7}{8} ; \frac{1}{4} \bar{x} \frac{7}{8}\right)$ \\
\hline$\ldots 22^{\mathrm{V}_{\mathrm{TC}}}{ }_{\mathrm{CC}} 1 \mathrm{x}$ & $\mathrm{IH}_{1} 22$ & $8(f) .2$. & $I+\left(x \frac{1}{4} \frac{1}{8} ; \bar{x} \frac{3}{4} \frac{1}{8} ; \frac{3}{4} \bar{x} \frac{7}{8} ; \frac{1}{4} x \frac{7}{8}\right)$ \\
\hline
\end{tabular}

\begin{tabular}{|lll|}
\hline$\overline{4} . . I_{2} P_{c 2} 1 \mathrm{x}$ & $I_{1} / \mathrm{acd}$ & $I+(x) 00 ; x \frac{1}{2} ; \bar{x} O \frac{1}{2} ; \bar{x} \frac{1}{2} O ;$ \\
& & $\frac{1}{4}, \frac{1}{4}-x, \frac{1}{4} ; \frac{3}{4}, \frac{3}{4}+x, \frac{1}{4} ; \frac{3}{4}, \frac{1}{4}+x, \frac{1}{4} ;$ \\
& $\left.\frac{1}{4}, \frac{3}{4}-x, \frac{1}{4}\right)$
\end{tabular}

\begin{tabular}{|c|c|c|c|}
\hline${ }^{4} 3 \cdot P_{c c}{ }^{V_{D I}} 1 x y$ & $\mathrm{P}_{3}$ & $4\left(a^{\prime}\right) 1$ & $x y 0 ; \bar{y} x \frac{3}{4} ; \bar{x} \bar{y} \frac{1}{2} ; y \bar{x} \frac{1}{4}$ \\
\hline
\end{tabular}

Table 22: Orthorhombic System

\begin{tabular}{|c|c|c|c|}
\hline$P$ & Pmmm & $1(a)$ mmm & 000 \\
\hline $\mathrm{P} 2 \mathrm{x}$ & $\operatorname{Prmmon}(j)$ & $\pm(x 00)$ & \\
\hline P $2 y 2 z$ & $\operatorname{Prmmon}(u)$ & $\pm(0, y, \pm z)$ & \\
\hline 2..P $2 x y$ & $\operatorname{Pccm}(q)$ & $\pm(x y 0) ; \pm\left(x \bar{y} \frac{1}{2}\right)$ & \\
\hline 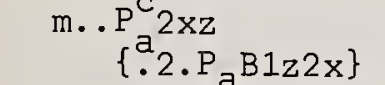 & $\operatorname{Prma}(i)$ & $\pm(x O z) ; \frac{1}{2} 00 \pm(x O \bar{z})$ & \\
\hline 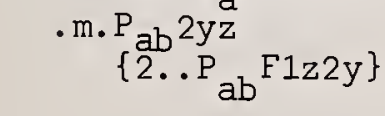 & $\operatorname{Crma} a(\mathrm{~m})$ & $C \pm\left(O y z ; \frac{1}{2} y \bar{z}\right)$ & \\
\hline
\end{tabular}




\begin{tabular}{|c|c|c|}
\hline $\mathrm{P} 2 \mathrm{x} 2 \mathrm{yz}$ & P222(u) & $x y z ; x \bar{y} \bar{z} ; \bar{x} y \bar{z} ; \bar{x} \bar{y} z$ \\
\hline P $2 \times 2 y 2 z$ & $\operatorname{Pnmm}(\alpha)$ & $\pm(x, y, \pm z ; x, \bar{y}, \pm z)$ \\
\hline $\begin{aligned} \cdots & m P \\
& \left\{\varepsilon^{2 x 2 y z}\right. \\
2 & 2 x y 2 z\}\end{aligned}$ & $\operatorname{Pccm}\left(x^{\prime}\right)$ & $x y z ; x \bar{y} \bar{z} ; \bar{x} y \bar{z} ; \bar{x} \bar{y} z ; 00 \frac{1}{2}+(x y \bar{z} ; x \bar{y} z ; \bar{x} y z ; \bar{x} \bar{y} \bar{z})$ \\
\hline $\begin{array}{l}\mathrm{m} . \mathrm{P}_{\mathrm{a}} 2 \mathrm{xz} 2 \mathrm{y} \\
\{.2 . \mathrm{P} \quad \mathrm{B} 1 \mathrm{z} 2 \mathrm{x} 2 \mathrm{y}\}\end{array}$ & Pmma (Z) & $\pm(x, \pm y, z) ; \frac{1}{2} 00 \pm(x, \pm y, \bar{z})$ \\
\hline 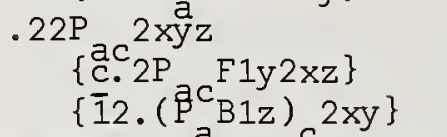 & $\operatorname{Pcca}(f)$ & $\pm(x y z) ; \frac{1}{2} 00 \pm(\bar{x} \bar{y} z) ; 00 \frac{1}{2} \pm(\bar{x} y \bar{z}) ; \frac{1}{2} O \frac{1}{2} \pm(x \bar{y} \bar{z})$ \\
\hline 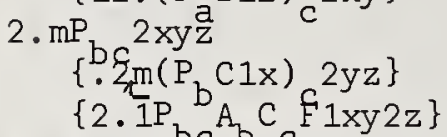 & $\operatorname{Pbcm}(e)$ & $\pm(x y z) ; O \frac{1}{2} O \pm(x \bar{y} \bar{z}) ; O O \frac{1}{2} \pm(x y \bar{z}) ; 0 \frac{1}{2} \frac{1}{2} \pm(x \bar{y} z)$ \\
\hline 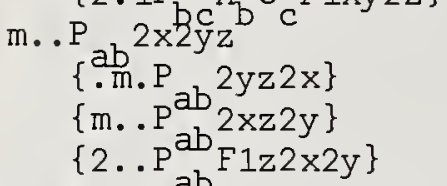 & Chma $\left(0^{\prime}\right)$ & $C+\left\{x y z ; x \bar{y} \bar{z} ; \bar{x} y \bar{z} ; \bar{x} \bar{y} z ; \frac{1}{2} 00+(\bar{x} y z ; \bar{x} \bar{y} \bar{z} ; x y \bar{z} ; x \bar{y} z)\right\}$ \\
\hline 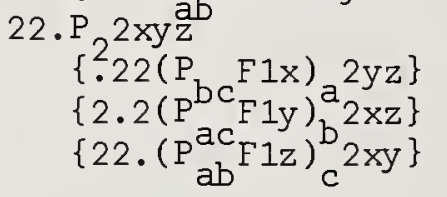 & $\operatorname{Ibca}(f)$ & $I+\left\{ \pm(x y z) ; \frac{1}{2} 00 \pm(\bar{x} y \bar{z}) ; 0 \frac{1}{2} 0 \pm(\bar{x} \bar{y} z) ; 00 \frac{1}{2} \pm(x \bar{y} \bar{z})\right\}$ \\
\hline
\end{tabular}

\begin{tabular}{|c|c|c|}
\hline Cmmm & $2(a) m m m$ & $000 ; \frac{1}{2} \frac{1}{2} 0$ \\
\hline $\begin{array}{l}C 2 x \\
C 2 z\end{array}$ & $\begin{array}{l}\operatorname{Crmm}(g) \\
\operatorname{Crmm}(k)\end{array}$ & $\begin{array}{l}C \pm(x 00) \\
C \pm(00 z)\end{array}$ \\
\hline $\mathrm{C} 2 \mathrm{y} 2 \mathrm{z}$ & $\operatorname{Crmm}(n)$ & $C \pm(0, y, \pm z)$ \\
\hline $\mathrm{C} 2 \mathrm{x} 2 \mathrm{y}$ & $C m m m(p)$ & $C \pm(x, \pm y, 0)$ \\
\hline $.2 . \mathrm{B} 2 \mathrm{yz}$ & $\operatorname{Prma}(h)$ & $\pm(O y z) ; \frac{1}{2} O \frac{1}{2} \pm(O y \bar{z})$ \\
\hline b..c2xy & $\operatorname{Pbam}(g)$ & $\pm(x y 0) ; \frac{1}{2} \frac{1}{2} 0 \pm(\bar{x} y 0)$ \\
\hline $\begin{array}{l}\cdot \text { - . C } 2 y z \\
\left\{\mathcal{F}^{2} \cdot \mathrm{C} \text { F } 1 \mathrm{y} 2 \mathrm{z}\right\}\end{array}$ & $\operatorname{Cmcm}(f)$ & $C+\left\{ \pm(0 y z) ; 00 \frac{1}{2} \pm(0 y \bar{z})\right\}$ \\
\hline n.. C F $2 x y^{-2}$ & $\operatorname{Cccm}(Z)$ & $C \pm\left(x y 0 ; x \bar{y} \frac{1}{2}\right)$ \\
\hline $\mathrm{b} . . \mathrm{c}^{\mathrm{c}_{2} \mathrm{xy}}$ & $\operatorname{Ibam}(j)$ & $I \pm\left(x y 0 ; x \bar{y} \frac{1}{2}\right)$ \\
\hline $\begin{aligned} 2 . & \mathrm{B}_{b}^{\mathrm{C}} 2 \mathrm{yz} \\
& \left\{.2 \cdot \mathrm{B}_{\mathrm{b}} \mathrm{A}_{\mathrm{a}} 1 \mathrm{z} 2 \mathrm{y}\right\}\end{aligned}$ & $\operatorname{Irma} a(h)$ & $I+\left\{ \pm(O y z) ; O \frac{1}{2} O \pm(O \bar{y} z)\right\}$ \\
\hline $\mathrm{C} 2 \mathrm{x} 2 \mathrm{yz}$ & $C 222(2)$ & $C+(x y z ; x \bar{y} \bar{z} ; \bar{x} y \bar{z} ; \bar{x} \bar{y} z)$ \\
\hline$c 2 x 2 y 2 z$ & $C m m m(r)$ & $C \pm(x, y, \pm z ; x, \bar{y}, \pm z)$ \\
\hline b..c $2 \times 2 y z$ & $\operatorname{Pban}(m)$ & $x y z ; x \bar{y} z ; \bar{x} y \bar{z} ; \bar{x} \bar{y} z ; \frac{1}{2} \frac{1}{2} O+(\bar{x} y z ; \bar{x} \bar{y} \bar{z} ; x y \bar{z} ; x \bar{y} z)$ \\
\hline 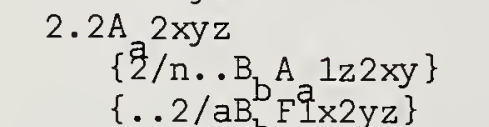 & Pnna(e) & $\pm(x y z) ; O \frac{1}{2} \frac{1}{2} \pm(x \bar{y} \bar{z}) ; \frac{1}{2} 00 \pm(\bar{x} \bar{y} z) ; \frac{1}{1} \frac{1}{2} \frac{1}{n} \pm(\bar{x} y \bar{z})$ \\
\hline .2. $32 \mathrm{yz} 2 \mathrm{x}$ & $\operatorname{Prma}(i)$ & $\pm( \pm x, y, z) ; \frac{1}{2} O \frac{1}{2} \pm( \pm x, y, \bar{z})$ \\
\hline b..c $2 x y 2 z$ & $\operatorname{Pbam}(i)$ & $\pm(x, y, \pm z) ; \frac{1}{2} \frac{1}{2} O \pm(\bar{x}, y, \pm z)$ \\
\hline $\begin{array}{l}\mathrm{b} 2 \cdot \mathrm{C}_{\mathrm{C}} 2 \mathrm{xyz} \\
\left\{\text { b. } 2{ }_{1} \mathrm{C}_{\mathrm{F}} \mathrm{F} 1 \mathrm{y} 2 \mathrm{xz}\right\}\end{array}$ & $\operatorname{Pbcn}(d)$ & $\pm(x y z) ; \frac{1}{2} \frac{1}{2} O \pm(\bar{x} y z) ; O O \frac{1}{2} \pm(\bar{x} y \bar{z}) ; \frac{1}{2} \frac{1}{2} \frac{1}{2} \pm(x y \bar{z})$ \\
\hline - $\operatorname{maB}_{\mathrm{b}} 2 \mathrm{xyz}$ & Pnma (d) & $\pm(x y z) ; \frac{1}{2} O \frac{1}{2} \pm(x y \bar{z}) ; O \frac{1}{2} O \pm(x \bar{y} z) ; \frac{1}{2} \frac{1}{2} \frac{1}{2} \pm(x \bar{y} \bar{z})$ \\
\hline $\begin{array}{l}\text {.n. c } 2 \mathrm{yz}^{2 \mathrm{x}} \text { a } \\
\text { n.. c } 2 \mathrm{x} 2 \mathrm{yz}\end{array}$ & $\begin{array}{l}\operatorname{Cmem}(h) \\
\operatorname{Cccm}\left(m^{\prime}\right)\end{array}$ & $\begin{array}{l}C+\left\{ \pm( \pm x, y, z) ; \frac{1}{2} \frac{1}{2} \pm( \pm x, \bar{y}, z)\right\} \\
C+\left\{x y z ; x \bar{y} \bar{z} ; \bar{x} y \bar{z} ; \bar{x} \bar{y} z ; \frac{1}{2} \frac{1}{2} \frac{1}{2}+(\bar{x} y z ; \bar{x} \bar{y} \bar{z} ; x y \bar{z} ; x \bar{y} z)\right\}\end{array}$ \\
\hline 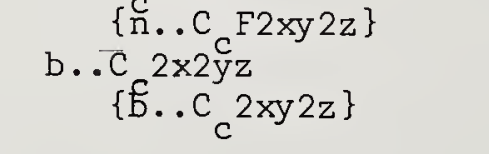 & $\operatorname{Ibam}\left(k^{\prime}\right)$ & $I+\left\{x y z ; x \bar{y} \bar{z} ; \bar{x} y \bar{z} ; \bar{x} \bar{y} z ; \frac{1}{2} \frac{1}{2} O+(\bar{x} y z ; \bar{x} \bar{y} \bar{z} ; x y \bar{z} ; x \bar{y} z)\right\}$ \\
\hline
\end{tabular}


$\begin{aligned} .2 . & \mathrm{B}_{2} 2 \mathrm{yz} 2 \mathrm{x} \\ & \{2 . \mathrm{A} 2 \mathrm{Am} 2 \mathrm{y}\} \\ & \left\{.2 \cdot \mathrm{B}_{\mathrm{B}}^{\mathrm{A} a} 1 \mathrm{z} 2 \mathrm{x} 2 \mathrm{y}\right\}\end{aligned}$

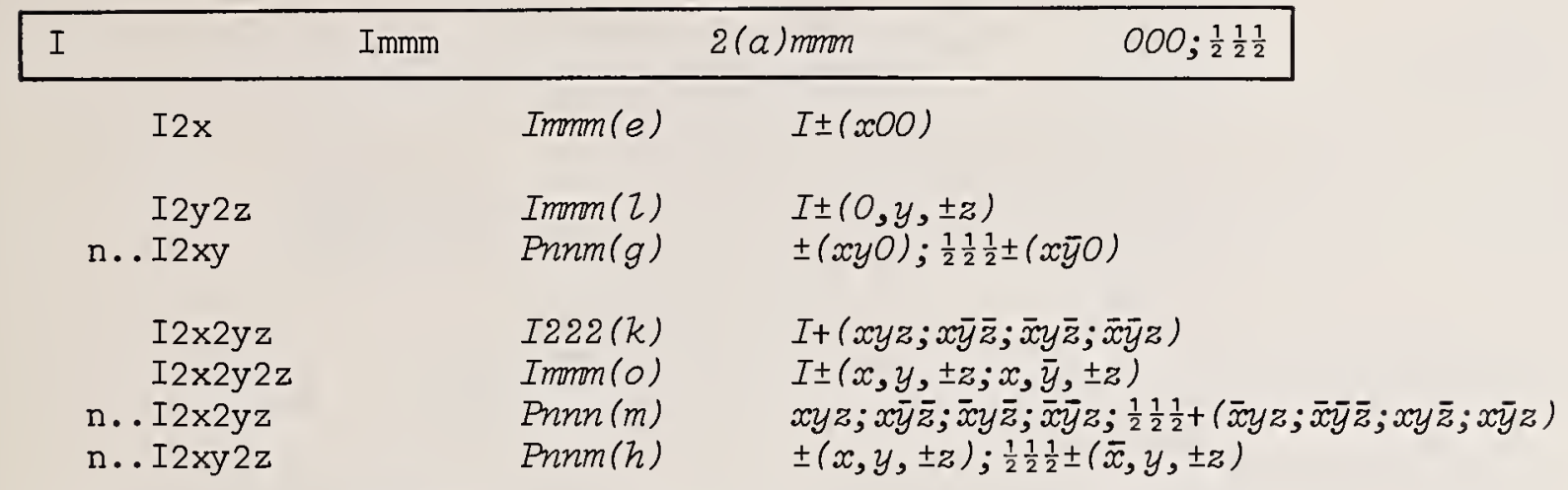

\begin{tabular}{|c|c|c|c|}
\hline $\mathrm{F}$ & Fmmm & $4(a)$ mrnm & $000 ; 0 \frac{1}{2} \frac{1}{2} \mathrm{~K}$ \\
\hline
\end{tabular}

\begin{tabular}{|c|c|c|}
\hline $\mathrm{F} 2 \mathrm{x}$ & Frnmm $(g)$ & $F \pm(x 00)$ \\
\hline $\begin{array}{l}\text { F2y } 2 z \\
\text {.2. F2yz }\end{array}$ & $\begin{array}{l}\operatorname{Frmm}(m) \\
\operatorname{Cmea}(f)\end{array}$ & $\begin{array}{l}F \pm(O, y, \pm z) \\
C+\left\{ \pm(O y z) ; \frac{1}{2} O \frac{1}{2} \pm(O y \bar{z})\right\}\end{array}$ \\
\hline $\begin{array}{l}\text { F2x2yz } \\
\text { F2x2y2z } \\
\text { c. } 2 \mathrm{~F} 2 x y z \\
\quad\left\{\overline{1} 21 \cdot(\mathrm{CI} 1 \mathrm{z}){ }_{\mathrm{c}} 2 \mathrm{xy}\right\} \\
\text { bc. } \mathrm{F} 2 \mathrm{xyz} \\
\text {.2. F } 2 \mathrm{yz} 2 \mathrm{x} \\
\text { c. } \mathrm{F} 2 \mathrm{x} 2 \mathrm{yz}\end{array}$ & $\begin{array}{l}\operatorname{Fr22}(k) \\
\operatorname{Ermm}(p) \\
\operatorname{Pccn}(e) \\
\operatorname{Pbca}(c) \\
\operatorname{Cmca}(g) \\
\operatorname{Ccca}(i)\end{array}$ & $\begin{array}{l}F+(x y z ; x \bar{y} \bar{z} ; \bar{x} y \bar{z} ; \bar{x} \bar{y} z) \\
F \pm(x, y, \pm z ; x, \bar{y}, \pm z) \\
\pm(x y z) ; O \frac{1}{2} \frac{1}{2} \pm(x \bar{y} z) ; \frac{1}{2} O \frac{1}{2} \pm(\bar{x} y z) ; \frac{1}{2} \frac{1}{2} O \pm(\bar{x} \bar{y} z) \\
\pm(x y z) ; O \frac{1}{2} \frac{1}{2} \pm(x \bar{y} z) ; \frac{1}{2} O \frac{1}{2} \pm(\bar{x} \bar{y} z) ; \frac{1}{2} \frac{1}{2} O \pm(\bar{x} y z) \\
C+\left\{ \pm( \pm x, y, z) ; \frac{1}{2} O \frac{1}{2} \pm( \pm x, y, \bar{z})\right\} \\
C+\left\{x y z ; x \bar{y} \bar{z} ; \bar{x} y \bar{z} ; \bar{x} \bar{y} z ; \frac{1}{2} O \frac{1}{2}+(\bar{x} y z ; \bar{x} \bar{y} \bar{z} ; x y \bar{z} ; x \bar{y} z)\right\}\end{array}$ \\
\hline
\end{tabular}

\begin{tabular}{|c|c|c|c|}
\hline D & Fddd & $8(a) 222$ & $E+\left(000 ; \frac{1}{4} \frac{1}{4} \frac{1}{4}\right)$ \\
\hline
\end{tabular}

$\begin{array}{lll}\text { D2x } & F d d d(e) & F+\left\{ \pm(x 00) ; \frac{1}{4} \frac{1}{4} \frac{1}{4} \pm(x 00)\right\} \\ \text { d. . D2xy } & F d d 2\left(b^{\prime}\right) & F+\left\{ \pm(x y 0) ; \frac{1}{4} \frac{1}{4} \frac{1}{4} \pm(\bar{x} y 0)\right\} \\ \text { d. D2x } 2 y z & F d d d(h) & F+\left\{x y z ; x \bar{y} z ; \bar{x} y \bar{z} ; \bar{x} \bar{y} z ; \frac{1}{4} \frac{1}{4} \frac{1}{4}+(\bar{x} y z ; \bar{x} \bar{y} \bar{z} ; x y \bar{z} ; x \bar{y} z)\right\}\end{array}$

\begin{tabular}{|llll|}
\hline $\mathrm{T}$ & Fddd & $16(c) \overline{1}$ & $F+\left(\frac{1}{8} \frac{1}{8} \frac{1}{8} ; \frac{1}{8} \frac{3}{8} \frac{3}{8} \mathrm{~K}\right)$ \\
\hline
\end{tabular}

\begin{tabular}{|c|c|c|c|c|}
\hline \multirow{2}{*}{\multicolumn{2}{|c|}{$\frac{\cdot 2 \cdot \mathrm{P}_{\mathrm{a}} \mathrm{B} 1 \mathrm{z}}{\cdot 2 \cdot \mathrm{P}_{\mathrm{a}}^{\mathrm{B} 1 \mathrm{z} 2 \mathrm{y}}}$}} & \multicolumn{2}{|r|}{$2\left(e^{\prime}\right) m m 2$} & \multirow[t]{2}{*}{$00 z ; \frac{1}{2} O \bar{z}$} \\
\hline & & $\operatorname{Prmma}\left(k^{\prime}\right)$ & $0, \pm y$, & \\
\hline 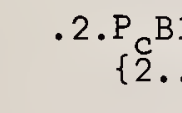 & $\mathrm{xz}\}$ & $P 222_{1}(e)$ & $x y z ; x$ & $\bar{z} ; \bar{x} \bar{y} z)$ \\
\hline
\end{tabular}




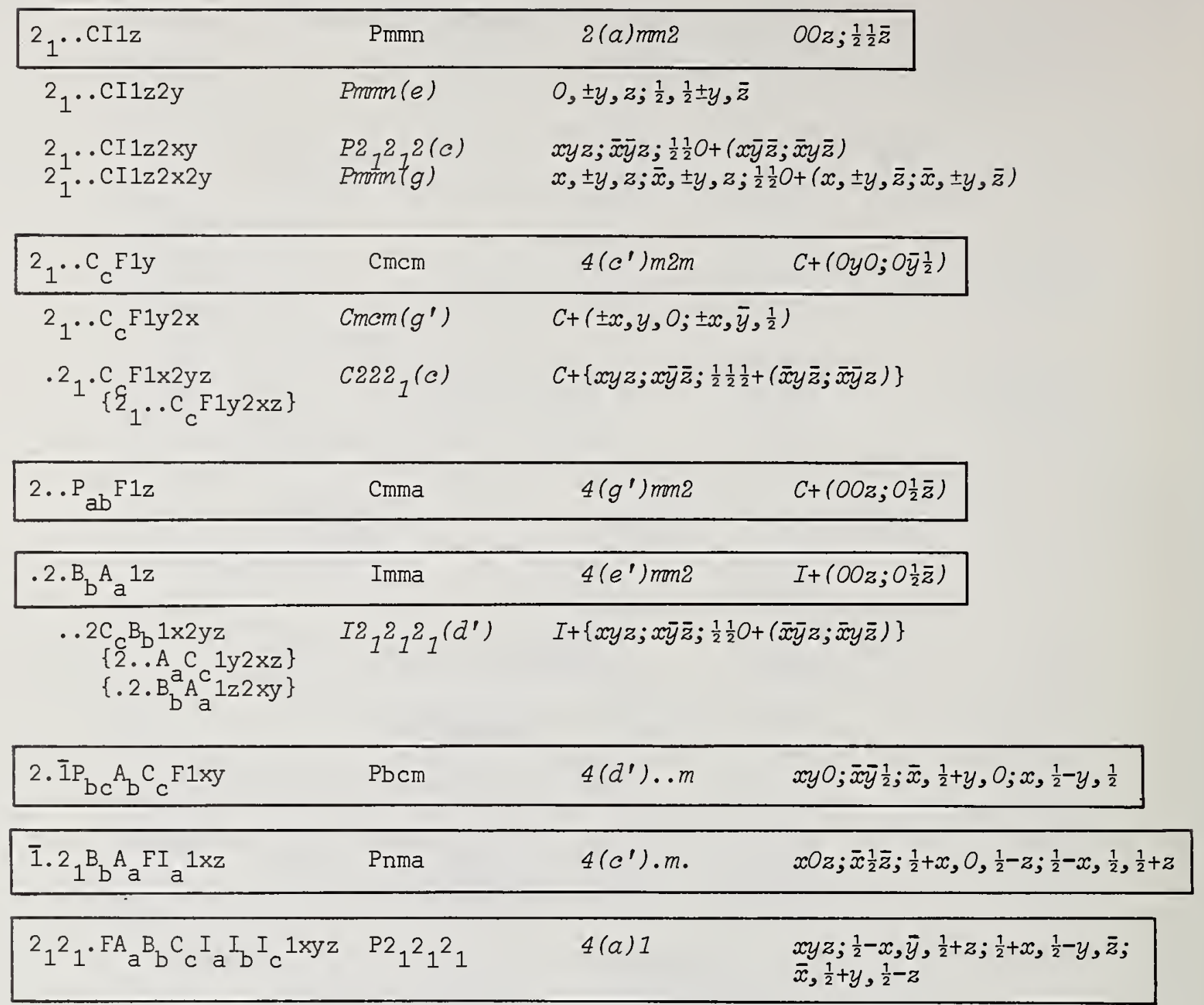

Table 23: Monoclinic System

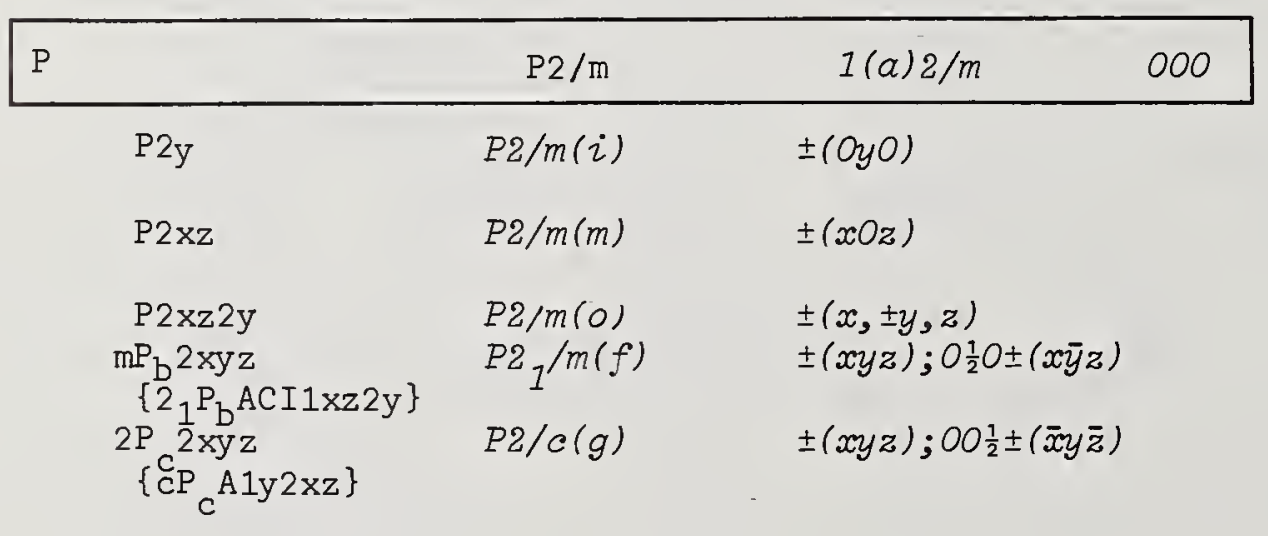




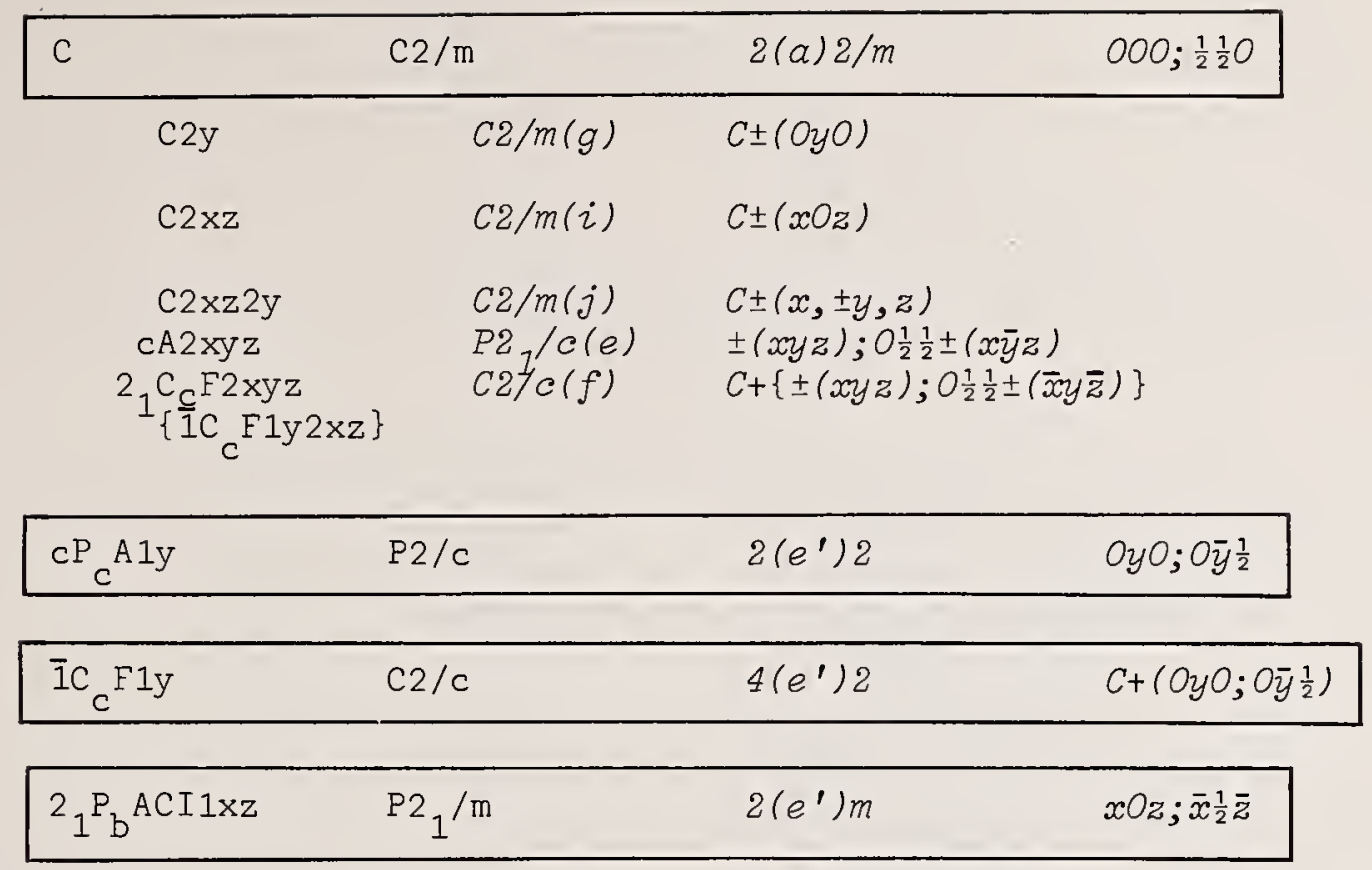

Table 24: Anorthic System

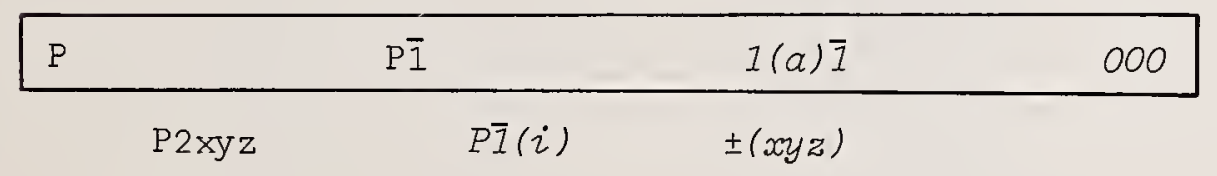



Occurrence of Lattice Complexes

Table 25

\begin{tabular}{|l|l|l|l|}
\hline $\begin{array}{l}\text { Lattice complex } \\
\text { in all its } \\
\text { representations }\end{array}$ & $\begin{array}{l}\text { Multi- } \\
\text { plicity }\end{array}$ & $\begin{array}{l}\text { Site } \\
\text { symmetry } \\
\text { (oriented) }\end{array}$ & $\begin{array}{l}\text { Occurrence: } \\
\text { space group, Wyckoff letter, } \\
\text { shift from standard representation }\end{array}$ \\
\hline
\end{tabular}

Cubic, invariant

\begin{tabular}{|c|c|c|c|}
\hline$P$ & 1 & $m 3 m$ & $\operatorname{Pm} 3 m(a),(b) \frac{1}{2} \frac{1}{2} \frac{1}{2}$ \\
\hline \multirow{16}{*}{ (a) $\mathrm{P}_{2}$} & \multirow{16}{*}{8} & $\overline{4} 3 m$ & $P \overline{4} 3 m(a),(b) \frac{1}{2} \frac{1}{2} \frac{1}{2}$ \\
\hline & & 432 & $P 432(a),(b) \frac{1}{2} \frac{1}{2} \frac{1}{2}$ \\
\hline & & $m 3$ & $\operatorname{Pm} 3(a),(b) \frac{1}{2} \frac{1}{2} \frac{1}{2}$ \\
\hline & & 23. & $P 23(a),(b) \frac{1}{2} \frac{1}{2} \frac{1}{2}$ \\
\hline & & $\overline{4} 3 m$ & $\operatorname{Em} 3 m(c) \div \frac{1}{4} \frac{1}{4}$ \\
\hline & & 432 & $\operatorname{Em} 3 c$ (a) $\frac{1}{4} \frac{1}{4} \frac{1}{4}$ \\
\hline & & $m 3$ & $\operatorname{Fm} 3 c(b)$ \\
\hline & & 23. & $F \overline{4} 3 c(a),(b) \frac{1}{4} \frac{1}{4} \frac{1}{4}$ \\
\hline & & & $F 432(c) \frac{1}{4} \frac{1}{4} \frac{1}{4}$ \\
\hline & & & Em3 (c) $\frac{1}{4} \frac{1}{4} \frac{1}{4}$ \\
\hline & &.$\overline{3} m$ & $\operatorname{Im} 3 m(c) \frac{1}{4} \frac{1}{4} \frac{1}{4}$ \\
\hline & & .32 & $\operatorname{Pm} 3 n(e) \frac{1}{4} \frac{1}{4} \frac{1}{4}$ \\
\hline & & & $I 432$ (c) $\frac{1}{4} \frac{1}{4} \frac{1}{4}$ \\
\hline & &.$\overline{3}$ & $\operatorname{Pn} 3 n(c) \frac{1}{4} \frac{1}{4} \frac{1}{4}$ \\
\hline & & & $\operatorname{Im} 3$ (c) $\frac{1}{4} \frac{1}{4} \frac{1}{4}$ \\
\hline & & & $\operatorname{Ia} 3(a),(b) \div \frac{1}{4} \frac{1}{4}$ \\
\hline \multirow[t]{11}{*}{ I } & \multirow[t]{11}{*}{2} & $m 3 m$ & $\operatorname{Im} 3 m(a)$ \\
\hline & & $\overline{4} 3 m$ & $\operatorname{Pn} 3 m(a)$ \\
\hline & & & $\operatorname{I\Phi } 3 m(a)$ \\
\hline & & 432 & $\operatorname{Pn} 3 n(a)$ \\
\hline & & & $I 432(a)$ \\
\hline & & m3. & $\operatorname{Pm} 3 n(a)$ \\
\hline & & & $\operatorname{Im} 3$ (a) \\
\hline & & 23. & $P \overline{4} 3 n(a)$ \\
\hline & & & $P 4232(a)$ \\
\hline & & & $\operatorname{Pn} 3$ (a) \\
\hline & & & I23 (a) \\
\hline
\end{tabular}

$\left({ }^{a}\right) 200 / 020 / 002$. From standard representation to cell. 


\begin{tabular}{|c|c|c|c|}
\hline$\left.{ }^{a}\right) I_{2}$ & 16 & $\begin{array}{l}23 \\
. \overline{3}\end{array}$ & $\begin{array}{l}E d 3 c(a) \\
\operatorname{Ia} 3 d(a)\end{array}$ \\
\hline $\mathrm{J}$ & 3 & $\begin{array}{l}4 / m m . m \\
\overline{4} 2 . m \\
42.2 \\
m m m . . \\
222 . . \\
\overline{4} m .2 \\
4 / m . . \\
\overline{4} . . \\
m . m m \\
2.22 \\
2 / m . .\end{array}$ & $\begin{array}{l}P m 3 m(c),(d) \frac{1}{2} \frac{1}{2} \frac{1}{2} \\
P \overline{4} 3 m(c),(d) \frac{1}{2} \frac{1}{2} \frac{1}{2} \\
P 432(c),(d) \frac{1}{2} \frac{1}{2} \frac{1}{2} \\
P m 3(c),(d) \frac{1}{2} \frac{1}{2} \frac{1}{2} \\
P 23(c),(d) \frac{1}{2} \frac{1}{2} \frac{1}{2} \\
F m 3 c(c) \frac{1}{4} \frac{1}{4} \frac{1}{4} \\
F m 3 c(d) \\
F \overline{4} 3 c(c),(d) \frac{1}{4} \frac{1}{4} \frac{1}{4} \\
F m 3 m(d) \\
F 432 \text { (d) } \\
F m 3 \text { (d) }\end{array}$ \\
\hline$\left(^{a}\right) F_{2}$ & 32 & $\begin{array}{l}m 3 m \\
\overline{4} 3 m \\
432 \\
m 3 \\
23 \\
. \overline{3} m \\
.32 \\
. \overline{3} \\
.32 \\
. \overline{3}\end{array}$ & 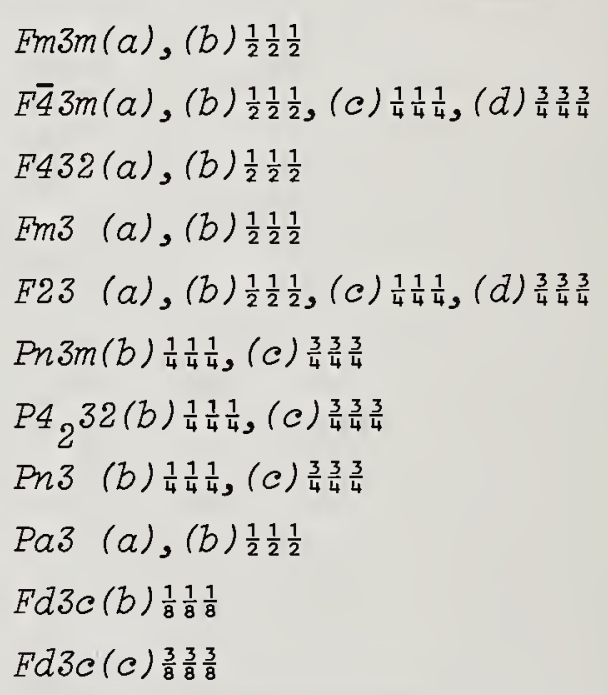 \\
\hline$\frac{{ }^{+} \mathrm{Y}}{\left({ }^{\mathrm{b}}\right)-\mathrm{Y}}$ & 4 & $\begin{array}{l}.32 \\
.32\end{array}$ & $\begin{array}{l}P 4_{3} 32(a),(b) \frac{1}{2} \frac{1}{2} \frac{1}{2} \\
P 4_{1} 32(a),(b) \frac{1}{2} \frac{1}{2} \frac{1}{2}\end{array}$ \\
\hline $\mathrm{W}$ & 6 & $\begin{array}{l}\overline{4} m .2 \\
\overline{4} . . \\
2.22\end{array}$ & $\begin{array}{l}P m 3 n(c),(d) \frac{1}{2} \frac{1}{2} \frac{1}{2} \\
P \overline{4} 3 n(c) \frac{1}{2} \frac{1}{2} \frac{1}{2},(d) \\
P 4232(e),(f) \frac{1}{2} \frac{1}{2} \frac{1}{2}\end{array}$ \\
\hline$J *$ & 6 & $\begin{array}{l}4 / m m \cdot m \\
\overline{4} 2 \cdot m\end{array}$ & $\begin{array}{l}\operatorname{Im} 3 m(b) \\
\operatorname{Pn} 3 m(d)\end{array}$ \\
\hline
\end{tabular}

$\left({ }^{a}\right) 200 / 020 / 002$. ( $\left.{ }^{b}\right) \overline{1} 00 / 0 \overline{1} 0 / 00 \overline{1}$. 


\begin{tabular}{|c|c|c|c|}
\hline$\left(^{\mathrm{a}}\right) \mathrm{J}{ }_{2}$ & 48 & $\begin{array}{l}42.2 \\
\text { mmm. } \\
222 .\end{array}$ & 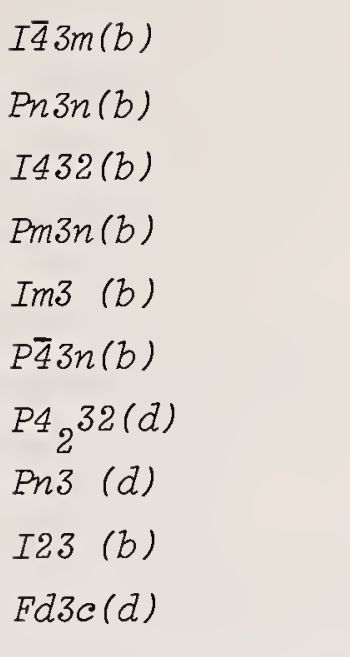 \\
\hline D & 8 & $\begin{array}{l}\overline{4} 3 m \\
23\end{array}$ & $\begin{array}{l}F d 3 m(a),(b) \frac{1}{2} \frac{1}{2} \frac{1}{2} \\
F 4_{1} 32(a),(b) \frac{1}{2} \frac{1}{2} \frac{1}{2} \\
F d 3(a),(b) \frac{1}{2} \frac{1}{2} \frac{1}{2}\end{array}$ \\
\hline$\frac{+_{Y *} \mid}{\left({ }^{b}\right)-Y^{*}}$ & $\begin{array}{l}8 \\
8\end{array}$ & $\begin{array}{l}.32 \\
.32\end{array}$ & $\begin{array}{l}I 4_{1} 32(a) \\
I 4_{1} 32(b)\end{array}$ \\
\hline$W^{*}$ & 12 & $\begin{array}{l}\overline{4} m .2 \\
\overline{4} . . \\
2.22\end{array}$ & $\begin{array}{l}\operatorname{Im} 3 m(d) \\
\operatorname{Pn} 3 n(d) \\
I \overline{4} 3 m(d) \\
\operatorname{Pn} 3 m(f) \\
I 432(d)\end{array}$ \\
\hline$\frac{s}{\left({ }^{b}\right)^{\prime} s}$ & $\begin{array}{l}12 \\
12\end{array}$ & $\begin{array}{l}\overline{4} . . \\
\overline{4} .\end{array}$ & $\begin{array}{l}I \overline{4} 3 a(a) \\
I \overline{4} 3 d(b)\end{array}$ \\
\hline$\frac{t^{+} \mathrm{V}}{\left({ }^{\mathrm{b}}\right)-\mathrm{V}}$ & $\begin{array}{l}12 \\
12\end{array}$ & $\begin{array}{l}2.22 \\
2.22\end{array}$ & $\begin{array}{l}I 4_{1} 32(c) \\
I 4_{1} 32(d)\end{array}$ \\
\hline $\mathrm{T}$ & 16 & $\begin{array}{l}. \overline{3} m \\
.32 \\
. \overline{3}\end{array}$ & $\begin{array}{l}F d 3 m(c),(d) \frac{1}{2} \frac{1}{2} \frac{1}{2} \\
F 4_{1} 32(c),(d) \frac{1}{2} \frac{1}{2} \frac{1}{2} \\
F d 3 \quad(c),(d) \frac{1}{2} \frac{1}{2} \frac{1}{2}\end{array}$ \\
\hline$Y * *$ & 16 & .32 & $\operatorname{Ia} 3 d(b)$ \\
\hline
\end{tabular}

$\left({ }^{a}\right) 200 / 020 / 002$. $\left({ }^{b}\right) \overline{1} 00 / 0 \overline{1} 0 / 00 \overline{1}$. 


\begin{tabular}{|l|l|l|l|}
\hline S* & 24 & $\overline{4} .$. & $\operatorname{Ia3d}(d)$ \\
\hline $\mathrm{V*}$ & 24 & 2.22 & $\operatorname{Ia} d(c)$ \\
\hline
\end{tabular}

Cubic, univariant

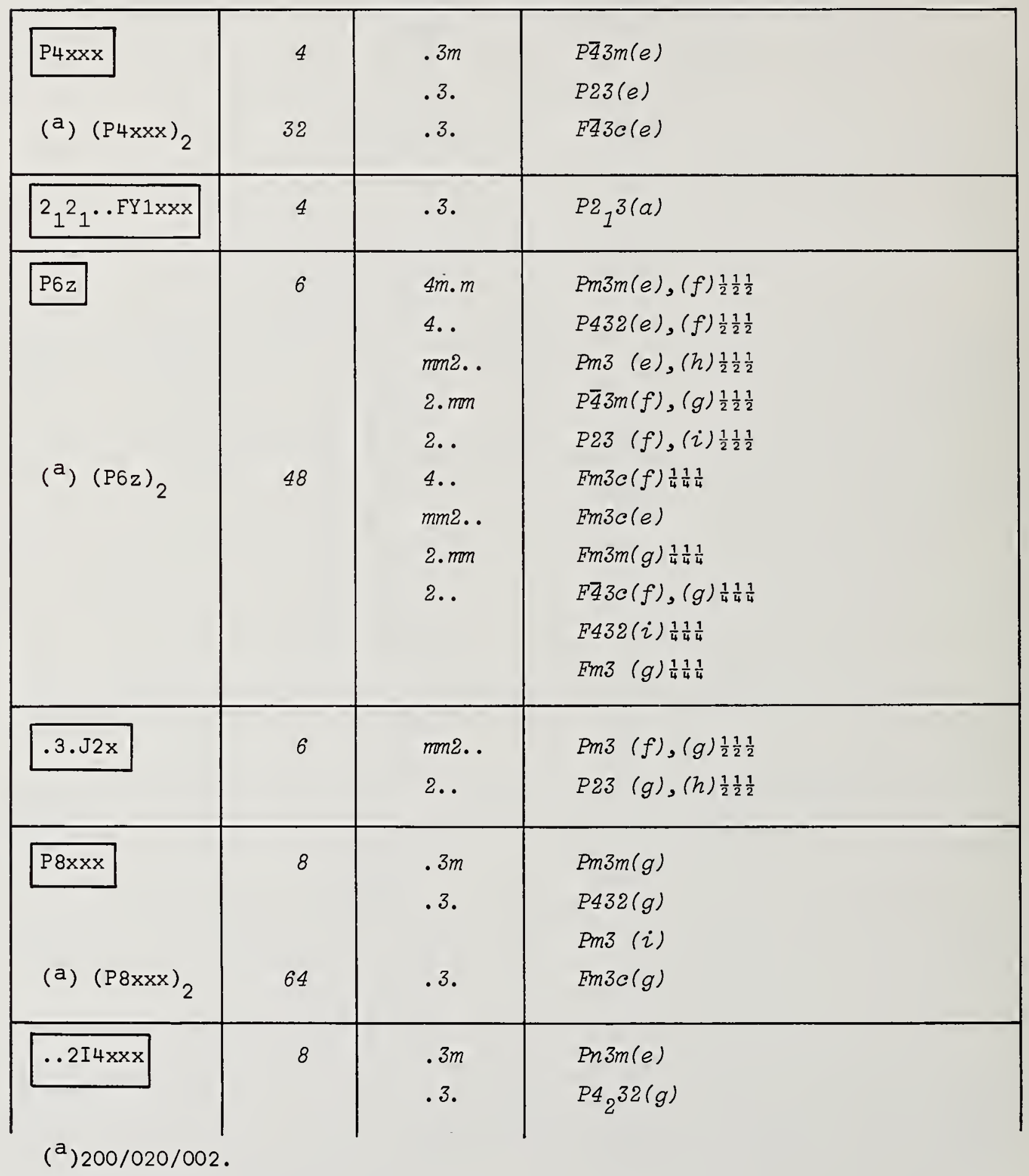




\begin{tabular}{|c|c|c|c|}
\hline ( $\left.{ }^{a}\right)(. .2 I 4 x x x)_{2}$ & 64 & .3 & $\begin{array}{l}\text { Pn3 (e) } \\
\text { Ed3c (e) }\end{array}$ \\
\hline $14 x \times x$ & 8 & $\begin{array}{l}.3 m \\
.3\end{array}$ & $\begin{array}{l}I \overline{4} 3 m(c) \\
P \overline{4} 3 n(e) \\
I 23(c)\end{array}$ \\
\hline$\frac{4}{{ }^{b}} \frac{{ }^{+}{ }^{Y} 2 \mathrm{xxx}}{4_{1} \ldots{ }^{-} \mathrm{Yxxx}}$ & $\begin{array}{l}8 \\
8\end{array}$ & $\begin{array}{l}.3 . \\
.3 .\end{array}$ & $\begin{array}{l}P 4_{3} 32(c) \\
P 4_{1} 32(c)\end{array}$ \\
\hline bc..F $2 x x x$ & 8 & .3 & $P a 3$ (c) \\
\hline${ }^{2}{ }_{1}{ }^{2}{ }_{1} \cdot P_{2} Y_{* 1 x x x}$ & 8 & .3 & $I 2_{1} 3(a)$ \\
\hline . 3.J4x & 12 & $\begin{array}{l}m m 2 . . \\
2 . .\end{array}$ & $\begin{array}{l}P m 3 m(h) \\
P \overline{4} 3 m(h) \\
P 432(h)\end{array}$ \\
\hline $\begin{array}{l}\text { P12xx } \\
\left({ }^{a}\right)(P 12 x x)_{2}\end{array}$ & $\begin{array}{l}12 \\
96\end{array}$ & $\begin{array}{l}m . m 2 \\
\ldots 2 \\
\ldots 2\end{array}$ & $\begin{array}{l}P m 3 m(i),(j) \frac{1}{2} \frac{1}{2} \frac{1}{2} \\
P 432(i),(j) \frac{1}{2} \frac{1}{2} \frac{1}{2} \\
E m 3 c(h) \frac{1}{4} \frac{1}{4} \frac{1}{4}\end{array}$ \\
\hline . 3. $\mathrm{W} 2 \mathrm{z}$ & 12 & $\begin{array}{l}m m 2 . . \\
2 . .\end{array}$ & $\begin{array}{l}\operatorname{Pm} 3 n(g),(h) \frac{1}{2} \frac{1}{2} \frac{1}{2} \\
P \overline{4} 3 n(g) \frac{1}{2} \frac{1}{2} \frac{1}{2},(h) \\
P 4{ }_{2} 32(i),(j) \frac{1}{2} \frac{1}{2} \frac{1}{2}\end{array}$ \\
\hline I6z & 12 & $\begin{array}{l}4 m . m \\
4 . . \\
m m 2 . \\
2 . m m \\
2 . .\end{array}$ & 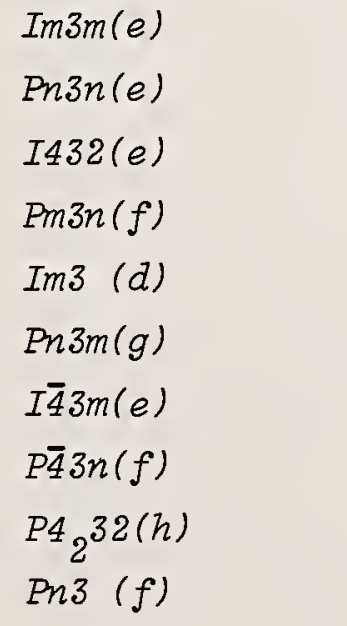 \\
\hline
\end{tabular}

$\left({ }^{a}\right) 200 / 020 / 002$. ( $\left.{ }^{b}\right) \overline{1} 00 / 0 \overline{1} 0 / 00 \overline{1}$. 


\begin{tabular}{|c|c|c|c|}
\hline$\left({ }^{a}\right)(I 6 z)_{2}$ & 96 & 2. & $\begin{array}{l}I 23(d) \\
F d 3 c(f)\end{array}$ \\
\hline${ }_{4}^{4} \ldots F 3 x \bar{x}$ & 12 & $\ldots 2$ & $P 4_{2} 32(k) \frac{1}{4} \frac{1}{4} \frac{1}{4},(Z) \frac{3}{4} \frac{3}{4} \frac{3}{4}$ \\
\hline $43 \ldots+Y 3 x \bar{x}$ & 12 & $\ldots 2$ & $P_{3} 32(d)$ \\
\hline$\left.{ }^{D}\right){ }_{1} \ldots Y 3 \times \bar{x}$ & 12 & $\ldots 2$ & $P_{1} 32(d)$ \\
\hline \multirow[t]{3}{*}{$.3 . J * 2 x$} & \multirow[t]{3}{*}{12} & \multirow{3}{*}{$\begin{array}{l}m m 2 . . \\
2 . .\end{array}$} & $\operatorname{Im} 3(e)$ \\
\hline & & & Pn3 (g) \\
\hline & & & I23 (e) \\
\hline $2{ }_{1}^{3 . S V} 1 z$ & 12 & $2 .$. & $I 2_{1} 3(b)$ \\
\hline \multirow[t]{5}{*}{$18 x \times x$} & \multirow[t]{5}{*}{16} & \multirow{5}{*}{$\begin{array}{l}.3 m \\
.3\end{array}$} & $\operatorname{Im} 3 m(f)$ \\
\hline & & & $\operatorname{Pn} 3 n(f)$ \\
\hline & & & $\operatorname{Pm} 3 n(i)$ \\
\hline & & & $I 432(f)$ \\
\hline & & & $\operatorname{Im} 3(f)$ \\
\hline \multirow[t]{2}{*}{$F 4 x \times x$} & \multirow[t]{2}{*}{16} & $.3 m$ & \multirow{2}{*}{$\begin{array}{l}F \overline{4} 3 m(e) \\
F 23(e)\end{array}$} \\
\hline & & .3 & \\
\hline$\overline{4} \ldots I_{2} Y * * 1 \times x \times$ & 16 & .3 & $I \overline{4} 3 d(c)$ \\
\hline $22 \ldots Y * 2 x x x$ & 16 & 3 & ${ }^{14}{ }_{1} 32(e)$ \\
\hline $22 \ldots P_{2} 2 x x x$ & 16 & .3 & $\operatorname{Ia} 3$ (c) \\
\hline .3. W4 $x \mathrm{x}$ & 24 & $\ldots 2$ & $\operatorname{Pm} 3 n(j)$ \\
\hline$\overline{4} \ldots F 6 \times \bar{x}$ & 24 & $\ldots 2$ & $\operatorname{Pn} 3 m(i) \frac{1}{4} \frac{1}{4} \frac{1}{4},(j) \frac{3}{4} \frac{3}{4} \frac{3}{4}$ \\
\hline \multirow[t]{4}{*}{ F6z } & \multirow[t]{4}{*}{24} & $4 m \cdot m$ & $\operatorname{Fm} 3 m(e)$ \\
\hline & & $4 \ldots$ & $F 432(e)$ \\
\hline & & $m m 2$. & $\operatorname{Em} 3$ (e) \\
\hline & & 2. $m m$ & $F \overline{4} 3 m(f),(g) \frac{1}{4} \frac{1}{4} \frac{1}{4}$ \\
\hline
\end{tabular}

$\left({ }^{\mathrm{a}}\right) 200 / 020 / 002$. $\left({ }^{\mathrm{b}}\right) \overline{1} 00 / 0 \overline{1} 0 / 00 \overline{1}$. 


\begin{tabular}{|c|c|c|c|}
\hline & & $2 .$. & $F 23(f),(g) \div \frac{1}{4} \frac{1}{4}$ \\
\hline $.3 . J * 4 x$ & 24 & $\begin{array}{l}m m 2 . . \\
2 . .\end{array}$ & $\begin{array}{l}\operatorname{Im} 3 m(g) \\
\operatorname{Pn} 3 n(g) \\
\operatorname{Pn} 3 m(h) \\
I \overline{4} 3 m(f) \\
I 432(g)\end{array}$ \\
\hline $\mathrm{I} 12 \mathrm{xx}$ & 24 & $\begin{array}{l}m \cdot m 2 \\
\ldots 2\end{array}$ & $\begin{array}{l}\operatorname{Im} 3 m(h) \\
\operatorname{Pn} 3 n(h) \\
\operatorname{I432(h)}\end{array}$ \\
\hline $.3 .52 z$ & 24 & 2. & $I \overline{4} 3 d(d)$ \\
\hline $4 . P_{2} 3 x \bar{x}$ & 24 & $\ldots 2$ & $I 432(i) \frac{1}{4} \frac{1}{4} \frac{1}{4}$ \\
\hline $.3 . \mathrm{V} 2 \mathrm{z}$ & 24 & 2. & ${ }^{I 4}{ }_{1} 32(f)$ \\
\hline$\frac{43}{\left({ }^{b}\right){ }^{+} Y * 3 x \bar{X}}$ & $\begin{array}{l}24 \\
24\end{array}$ & $\begin{array}{l}\ldots 2 \\
\ldots 2\end{array}$ & $\begin{array}{l}I 4_{1} 32(\mathrm{~h}) \\
{ }^{I 4}{ }_{1} 32(\mathrm{~g})\end{array}$ \\
\hline.$\overline{3} \cdot \mathrm{J}_{2} \mathrm{~S} * \mathrm{~V} * 1 \mathrm{x}$ & 24 & 2. & $\operatorname{Ia} 3$ (d) \\
\hline$F 8 \times x \times$ & 32 & $\begin{array}{l}.3 m \\
.3\end{array}$ & $\begin{array}{l}\operatorname{Fm3m}(f) \\
F 432(f) \\
\operatorname{Em} 3(f)\end{array}$ \\
\hline$\ldots 2 \mathrm{D} 4 \mathrm{xxx}$ & 32 & $\begin{array}{l}.3 m \\
.3\end{array}$ & $\begin{array}{l}F d 3 m(e) \\
F 4_{1} 32(e) \\
F d 3(e)\end{array}$ \\
\hline$\overline{4} . . Y * * 2 \mathrm{xxx}$ & 32 & .3 & $\operatorname{Ia} d(e)$ \\
\hline $\mathrm{F} 12 \mathrm{xx}$ & 48 & $\begin{array}{l}m . m 2 \\
\ldots 2\end{array}$ & $\begin{array}{l}F m 3 m(h),(i) \frac{1}{2} \frac{1}{2} \frac{1}{2} \\
F 432(g),(h) \frac{1}{2} \frac{1}{2} \frac{1}{2}\end{array}$ \\
\hline
\end{tabular}




\begin{tabular}{|c|c|c|c|}
\hline $\mathrm{D} 6 \mathrm{z}$ & 48 & $\begin{array}{l}\text { 2.mm } \\
2 . .\end{array}$ & $\begin{array}{l}F d 3 m(f) \\
F 4_{1} 32(f) \\
F d 3(f)\end{array}$ \\
\hline $4 \ldots P_{2} 6 x \bar{x}$ & 48 &. .2 & $\operatorname{Im} 3 m(i) \frac{1}{1} \frac{1}{4} \frac{1}{4}$ \\
\hline . $3.5 * 2 z$ & 48 & 2.. & $\operatorname{Ia} 3 d(f)$ \\
\hline$\overline{4} a . . Y * * 3 \times \bar{x}$ & 48 &. .2 & $\operatorname{Ia} 3 d(g)$ \\
\hline $22 \ldots T 3 \times \bar{x}$ & 48 &. .2 & $F^{4}{ }_{1}^{32(g)}$ \\
\hline$\overline{4} . . T 6 \times \bar{x}$ & 96 & .. 2 & $F d 3 m(h)$ \\
\hline$\overline{4} 2 \ldots F_{2} 3 x \bar{x}$ & 96 & .. 2 & $F d 3 c(g) \frac{1}{8} \frac{1}{8} \frac{1}{8}$ \\
\hline
\end{tabular}

Cubic, bivariant

\begin{tabular}{|l|l|l|l|}
\hline P6z2xx & 12 & $\ldots m$ & $P \overline{4} 3 m(i)$ \\
\hline P6z2x & 12 & $m .$. & $\operatorname{Pm} 3(j),(k) \frac{1}{2} \frac{1}{2} \frac{1}{2}$ \\
\hline P6z4x & 24 & $m .$. & $\operatorname{Pm} 3 m(k),(2) \frac{1}{2} \frac{1}{2} \frac{1}{2}$ \\
\hline P6z4xx & 24 & $\ldots m$ & $\operatorname{Pm} 3 m(m)$ \\
\hline. $.216 \mathrm{z} 2 \mathrm{x}$ & 24 & $m .$. & $\operatorname{Pm} 3 n(k)$ \\
\hline. $.2 \mathrm{I} 6 \mathrm{z} 2 \mathrm{xx}$ & 24 & $\ldots m$ & $\operatorname{Pn} 3 m(k)$ \\
\hline $\mathrm{I} 6 \mathrm{z} 2 \mathrm{xx}$ & 24 & $\ldots m$ & $\operatorname{I\overline {4}} 3 m(g)$ \\
\hline $\mathrm{I} 6 \mathrm{z} 2 \mathrm{x}$ & 24 & $m .$. & $\operatorname{Im} 3(g)$ \\
\hline
\end{tabular}




\begin{tabular}{|l|c|c|c|}
\hline$I 6 \mathrm{z} 4 \mathrm{x}$ & 48 & $m .$. & $\operatorname{Im} 3 m(j)$ \\
\hline $\mathrm{I} 6 \mathrm{z} 4 \mathrm{xx}$ & 48 & $\ldots m$ & $\operatorname{Im} 3 m(k)$ \\
\hline $\mathrm{F} 6 \mathrm{z} 2 \mathrm{xx}$ & 48 & $\ldots m$ & $F \overline{4} 3 m(h)$ \\
\hline $\mathrm{F} 6 \mathrm{z} 2 \mathrm{x}$ & 48 & $m$. & $\operatorname{Fm} 3(h)$ \\
\hline $\mathrm{F} 6 \mathrm{z} 4 \mathrm{x}$ & 96 & $m .$. & $\operatorname{Fm} 3 m(j)$ \\
\hline $\mathrm{F} 6 \mathrm{z} 4 \mathrm{xx}$ & 96 & $\ldots m$ & $\operatorname{Fm} 3 m(k)$ \\
\hline$\ldots 2 \mathrm{P}_{2} 6 \mathrm{z} 2 \mathrm{x}$ & 96 & $m .$. & $\operatorname{Fm} 3 c(i)$ \\
\hline$\ldots 2 \mathrm{D} 6 \mathrm{z} 2 \mathrm{xx}$ & 96 & $\ldots m$ & $\operatorname{Fd} 3 m(g)$ \\
\hline
\end{tabular}

Cubic, trivariant (site symmetry 1)

The 12-pointers:

\begin{tabular}{|l|l|}
\hline P6z2xy & P23 (j) \\
\hline $2{ }_{1}{ }_{1} \ldots$ FY1 1 xxx3yz & $P 2{ }_{1} 3(b)$ \\
\hline
\end{tabular}

The 24-pointers:

\begin{tabular}{|c|c|}
\hline P6z2xx2y & $P \overline{4} 3 m(j)$ \\
\hline ..cI6z2xy & $P \overline{4} 3 n(i)$ \\
\hline P6 z $4 x y$ & $P 432(k)$ \\
\hline$\ldots 2 I 6 z 2 x y$ & $P 4_{2} 32(\mathrm{~m})$ \\
\hline${ }^{4}{ }^{3}+^{+} Y 3 x \bar{x} 2 y z$ & ${ }^{P 4}{ }_{3} 32(e)$ \\
\hline (b) ${ }_{4} \ldots-Y 3 x \bar{x} 2 y z$ & $P 4_{1} 32(e)$ \\
\hline
\end{tabular}

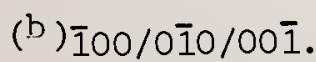

\begin{tabular}{|l|l|}
\hline P6z2x2y & Pm3 (l) \\
\hline $\mathrm{n} .$. I6z2xy & Pn3 (h) \\
\hline bc..F6xyz & Pa3 (d) \\
\hline I6z2xy & $I 23(f)$ \\
\hline $2{ }_{1}{ }_{1} \ldots P_{2} Y_{* * 1 \times x \times 3 y z}$ & $I 2_{1}^{3(c)}$ \\
\hline
\end{tabular}

The 48-pointers:

\begin{tabular}{|l|l|}
\hline P6z4x2y & $\operatorname{Pm} 3 m(n)$ \\
\hline $\mathrm{n} .1 \mathrm{I} 6 \mathrm{z} 4 \mathrm{xy}$ & $\operatorname{Pn} 3 n(i)$ \\
\hline$. .216 z 2 \times 2 \mathrm{y}$ & $\operatorname{Pm} 3 n(z)$ \\
\hline
\end{tabular}




\begin{tabular}{|c|c|}
\hline ..2I6z $2 x \times 2 y$ & $\operatorname{Pn} 3 m(Z)$ \\
\hline I6z2xx2y & $I \overline{4} 3 m(h)$ \\
\hline . $3 d S 4 x y z$ & $I \overline{4} 3 d(e)$ \\
\hline $16 z 4 x y$ & $I 432(j)$ \\
\hline $22 \ldots Y * 3 x \bar{x} 2 y z$ & $I 4_{1} 32(i)$ \\
\hline $16 z 2 x 2 y$ & $\operatorname{Im} 3(h)$ \\
\hline $22 \ldots P_{2} 6 x y z$ & $\operatorname{Ia3}(e)$ \\
\hline F6z2xy & F23 (h) \\
\hline $16 z 4 x 2 y$ & $\operatorname{Im} 3 m(Z)$ \\
\hline$\overline{4} a . . Y * * 3 x \bar{x} 2 y z$ & $\operatorname{Ia} 3 d(h)$ \\
\hline
\end{tabular}

\begin{tabular}{|l|l|}
\hline $.2 \mathrm{D} 6 \mathrm{z} 2 \mathrm{xx} 2 \mathrm{y}$ & $F d 3 m(i)$ \\
\hline $\mathrm{d} .2 \mathrm{I}_{2} 6 \mathrm{z} 2 \mathrm{xy}$ & $F d 3 c(h)$ \\
\hline
\end{tabular}

The 96-pointers:

\begin{tabular}{|l|c|}
\hline F6z2xx2y & $F \overline{4} 3 m(i)$ \\
\hline$\cdots \mathrm{nP}_{2} 6 \mathrm{z} 2 \mathrm{xy}$ & $F \overline{4} 3 c(h)$ \\
\hline $\mathrm{F} 6 \mathrm{z} 4 \mathrm{xy}$ & $F 432(j)$ \\
\hline$\ldots 2 \mathrm{D} 6 \mathrm{z} 2 \mathrm{xy}$ & $F 41^{32}(h)$ \\
\hline F6z2x2y & $F m 3(i)$ \\
\hline $\mathrm{d} . \mathrm{D} 6 \mathrm{z} 2 \mathrm{xy}$ & $F d 3(g)$ \\
\hline
\end{tabular}

The 192-pointers:

\begin{tabular}{|l|l|}
\hline F6z4x2y & Fm3m(Z) \\
\hline m..P $26 z 4 x y$ & $F m 3 c(j) \frac{1}{4} \frac{1}{4} \frac{1}{4}$ \\
\hline
\end{tabular}


Occurrence of Lattice Complexes

Table 26-27

\begin{tabular}{|l|l|l|l|}
\hline $\begin{array}{l}\text { Lattice complex } \\
\text { in all its } \\
\text { representations }\end{array}$ & $\begin{array}{l}\text { Multi- } \\
\text { plicity }\end{array}$ & $\begin{array}{l}\text { Site } \\
\text { symmetry } \\
\text { (oriented) }\end{array}$ & $\begin{array}{l}\text { Occurrence: } \\
\text { space group, Wyckoff letter, } \\
\text { shift from standard representation }\end{array}$ \\
\hline
\end{tabular}

Hexagonal and rhombohedral, invariant

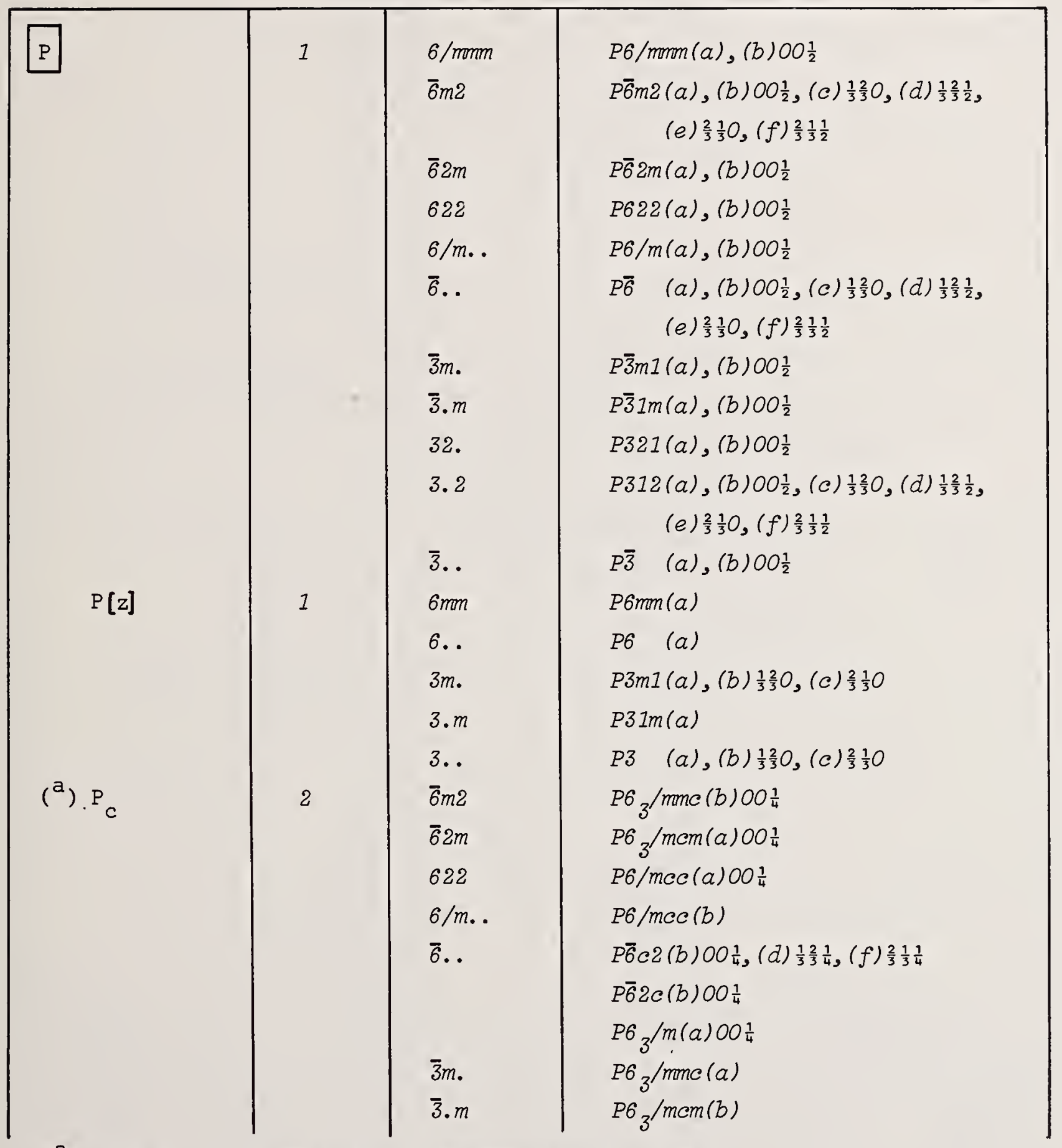

(a)100/010/002. From standard representation to cell. 


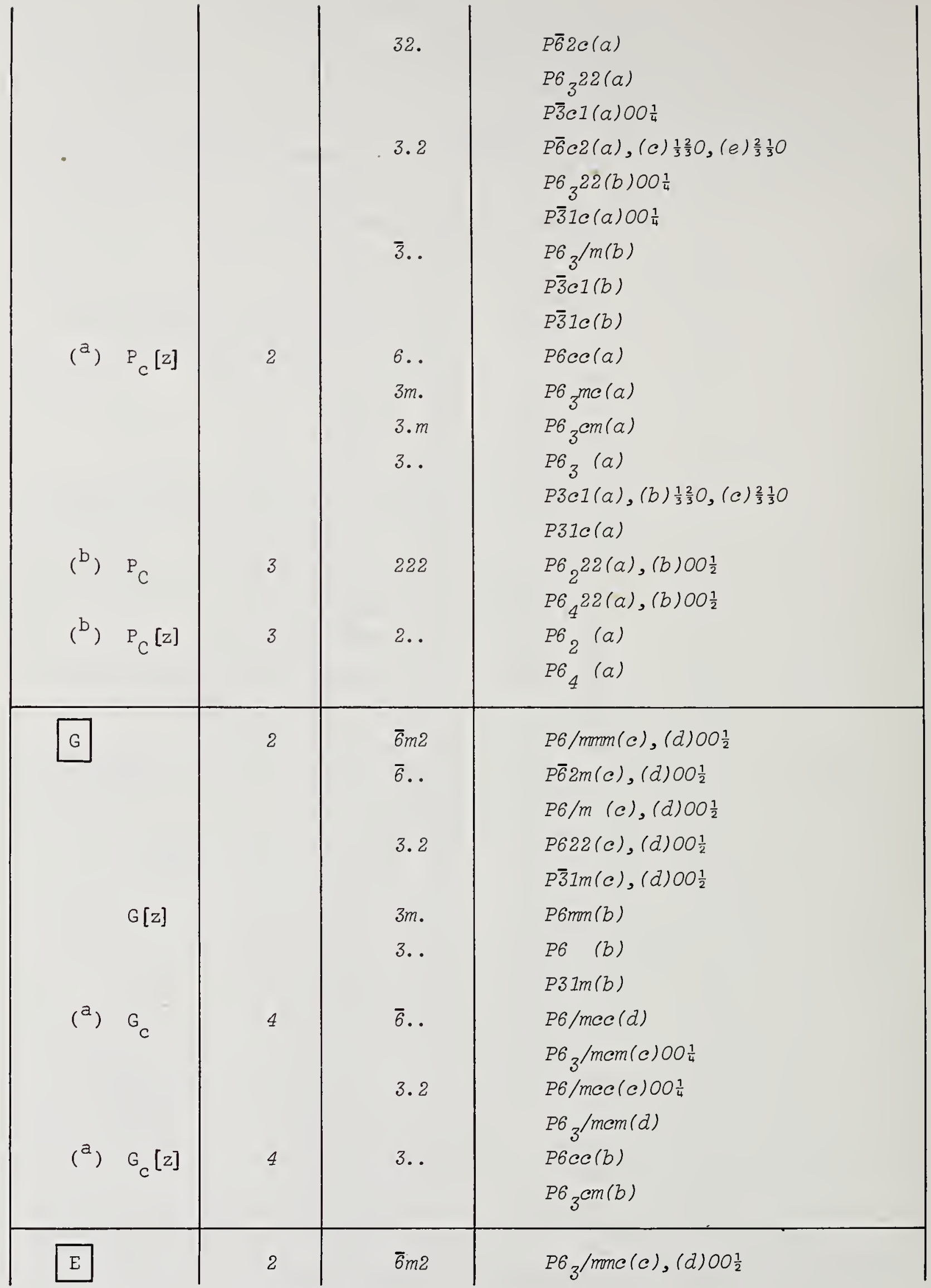

(a) $100 / 010 / 002 \cdot\left({ }^{b}\right) 100 / 010 / 003$. 


\begin{tabular}{|c|c|c|c|c|}
\hline & [z] & 2 & $\begin{array}{l}\overline{6} . . \\
3.2 \\
3 m . \\
3 . .\end{array}$ & $\begin{array}{l}P \overline{6} 2 c(c),(d) 00 \frac{1}{2} \\
P 6_{3} / m(c),(d) 00 \frac{1}{2} \\
P 6_{3} 22(c),(d) 00 \frac{1}{2} \\
P \overline{3} 1 c(c),(d) 00 \frac{1}{2} \\
P 6_{3} m c(b) \\
P 6_{3}(b) \\
P 31 c(b)\end{array}$ \\
\hline $\begin{array}{l}\left({ }^{c}\right) \\
\left({ }^{c}\right)\end{array}$ & $\begin{array}{l}{ }^{\prime} R_{c} \\
{ }^{\prime} R_{c}[z]\end{array}$ & 3 & $\begin{array}{l}\overline{3} m . \\
32 . \\
\overline{3} . . \\
3 m . \\
3 \ldots \\
32 . \\
\overline{3} . . \\
3 \ldots\end{array}$ & $\begin{array}{l}R \overline{3} m \quad(a),(b) 00 \frac{1}{2} \\
R 32 \quad(a),(b) 00 \frac{1}{2} \\
R \overline{3} \quad(a),(b) 00 \frac{1}{2} \\
R 3 m \quad(a) \\
R 3 \quad(a) \\
R \overline{3} c \quad(a) 00 \frac{1}{4} \\
R \overline{3} c \quad(b) \\
R 3 c \quad(a)\end{array}$ \\
\hline$\left({ }^{a}\right)$ & $\mathrm{N}_{\mathrm{C}}$ & 3 & $\begin{array}{l}m m m \\
222 \\
2 / m . . \\
.2 / m \\
\ldots 2 / m \\
\overline{1} \\
2 m m \\
2 . . \\
222 \\
2 / m . \\
.2 / m . \\
\ldots 2 / m \\
\overline{1} \\
\\
2 . .\end{array}$ & $\begin{array}{l}P 6 / m m m(f),(g) 00 \frac{1}{2} \\
P 622(f),(g) 00 \frac{1}{2} \\
P 6 / m(f),(g) 00 \frac{1}{2} \\
P \overline{3} m I(e),(f) 00 \frac{1}{2} \\
P \overline{3} 1 m(f),(g) 00 \frac{1}{2} \\
P \overline{3} \quad(e),(f) 00 \frac{1}{2} \\
P 6 m m(c) \\
P 6 \quad(c) \\
P 6 / m c c(f) 00 \frac{1}{4} \\
P 6 / m c c(g) \\
P 6{ }_{3} / m m c(g) \\
P 6{ }_{3} / m c m(f) \\
P 6{ }_{3} / m(g) \\
P \overline{3} \mathrm{c} 1(e) \\
P \overline{3} I c(g) \\
P 6 c c(c)\end{array}$ \\
\hline$\left.{ }^{d}{ }^{d}\right)$ & $\begin{array}{l}{ }^{+} Q[z] \\
-Q\end{array}$ & 3 & $\begin{array}{l}2.22 \\
2 . \\
222\end{array}$ & $\begin{array}{l}P 6_{2} 22(c),(d) 00 \frac{1}{2} \\
P 6_{2} \text { (b) } \\
P 6_{4} 22(c),(d) 00 \frac{1}{2}\end{array}$ \\
\hline
\end{tabular}

(a) $100 / 010 / 002$. ( $\left.{ }^{c}\right) 0 \overline{1} 0 / 110 / 002$. ( $\left.{ }^{d}\right) 100 / 010 / 00 \overline{1}$. 


\begin{tabular}{|l|l|l|l|}
$\left({ }^{\mathrm{d}}\right)-Q[z]$ & 3 & $2 .$. & $P 6_{4}(b)$ \\
\hline $\mathrm{M}$ & 9 & $.2 / m$. & $R \overline{3} m(d) 00 \frac{1}{2},(e)$ \\
\hline & & $\overline{1}$ & $R \overline{3}(d) 00 \frac{1}{2},(e)$ \\
$\left({ }^{c}\right){ }^{\prime}{ }_{C}$ & 18 & $\overline{1}$ & $R \overline{3} c(d)$ \\
\hline
\end{tabular}

Hexagonal and rhombohedral, univariant

\begin{tabular}{|c|c|c|c|}
\hline$\left.{ }^{\mathrm{b}}\right)(\mathrm{P} 2 \mathrm{z}) \mathrm{C}$ & 6 & $\begin{array}{l}6 m m \\
6 . . \\
3 m . \\
3 . m \\
3 . . \\
\\
6 . . \\
3 m . \\
3 . m \\
3 . .\end{array}$ & $\begin{array}{l}P 6 / m m m(e) \\
P 622(e) \\
P 6 / m(e) \\
P \overline{6} m 2(g),(h) \frac{1}{3} \frac{2}{3} 0,(i) \frac{2}{3} \frac{1}{3} O \\
P \overline{3} m 1(e) \\
P \overline{6} 2 m(e) \\
P \overline{3} 1 m(e) \\
P \overline{6} \quad(g),(h) \frac{1}{3} \frac{2}{3} O,(i) \frac{2}{3} \frac{1}{3} O \\
P 321(e) \\
P 312(g),(h) \frac{1}{3} \frac{2}{3} O,(i) \frac{2}{3} \frac{1}{3} O \\
P \overline{3} \quad(e) \\
P 6 / m c c(e) \\
P 6_{3} / m m c(e) \\
P 6_{3} / m c m(e) \\
P \overline{6} c 2(g),(h) \frac{1}{3} \frac{2}{3} O,(i) \frac{2}{3} \frac{1}{3} O \\
P \overline{6} 2 c(e) \\
P 6{ }_{3} 22(e) \\
P 6_{3} / m(e) \\
P \overline{3} c 1(e) \\
P \overline{3} 1 c(e) \\
P 6{ }_{2} 22(e) \\
P 6_{4} 22(e)\end{array}$ \\
\hline . 2.GE1z & 2 & $\begin{array}{c}3 m . \\
3 . .\end{array}$ & $\begin{array}{l}P \overline{3} m 1(d) \\
P 321(d) \\
P \overline{3} \quad \text { (d) }\end{array}$ \\
\hline
\end{tabular}

$\left({ }^{a}\right) 100 / 010 / 002$. ( $\left.{ }^{b}\right) 100 / 010 / 003 . \quad\left({ }^{c}\right) 0 \overline{1} 0 / 110 / 002$. ( $\left.{ }^{d}\right) 100 / 010 / 00 \overline{1}$. 


\begin{tabular}{|c|c|c|c|}
\hline$\left({ }^{a}\right)(.2 \cdot \operatorname{GE} 1 z){ }_{c}$ & 4 & $3 .$. & $P \overline{3} c 1(d)$ \\
\hline \multirow[t]{2}{*}{ P3x $\bar{x}$} & \multirow[t]{2}{*}{3} & $m m 2$ & $P \overline{6} m 2(j),(k) 00 \frac{1}{2}$ \\
\hline & &. .2 & $P 312(j),(k) 00 \frac{1}{2}$ \\
\hline$P 3 x \bar{x}[z]$ & \multirow[t]{2}{*}{3} &.$m$ & $P 3 m 1(d)$ \\
\hline$\left({ }^{a}\right)(P 3 x \bar{x})_{C}$ & & .. 2 & $P \bar{\sigma} c 2(j)$ \\
\hline \multirow[t]{2}{*}{$\mathrm{P} 3 \mathrm{x}$} & \multirow[t]{2}{*}{3} & $m 2 m$ & $P \overline{6} 2 m(f),(g) 0 O \frac{1}{2}$ \\
\hline & & .2. & $P 321(e),(f) 00 \frac{1}{2}$ \\
\hline \multirow{2}{*}{ (a) $\begin{aligned} & P 3 x\{z\} \\
(P 3 x) & \end{aligned}$} & \multirow{2}{*}{$\begin{array}{l}3 \\
6\end{array}$} & $\ldots m$ & $\operatorname{P3} \operatorname{Im}(c)$ \\
\hline & & .2 & $P \overline{6} 2 c(g)$ \\
\hline \multirow{2}{*}{$-\frac{2}{3} \frac{\mathrm{P}_{C}{ }^{+} \mathrm{Q} 1 \mathrm{x} \overline{\mathrm{x}}}{{ }_{1} \ldots \mathrm{P}_{\mathrm{C}} \mathrm{Q} 1 \mathrm{x} \overline{\mathrm{x}}}$} & 3 & $\ldots 2$ & $P 3{ }_{2}^{12(a) 00 \frac{2}{3},(b) 00 \frac{1}{6}}$ \\
\hline & 3 & $\ldots 2$ & $\ddot{P} 3_{1} 12(a) 00 \frac{1}{3},(b) 00 \frac{5}{6}$ \\
\hline \multirow{2}{*}{ 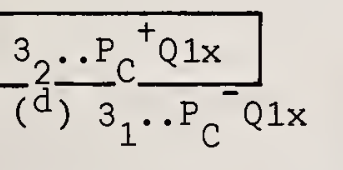 } & 3 & .2 & $P 3221(a) 00 \frac{2}{3},(b) 00 \frac{1}{6}$ \\
\hline & 3 & .2. & $P 31^{21(a) 00 \frac{1}{3},(b) 00 \frac{5}{6}}$ \\
\hline G2z & \multirow[t]{2}{*}{4} & $3 m$. & $P 6 /$ mmm $(h)$ \\
\hline \multirow{6}{*}{$\left({ }^{a}\right)(G 2 z)_{2}$} & & 3. & $P \overline{6} 2 m(h)$ \\
\hline & \multirow{5}{*}{8} & & $P 622(h)$ \\
\hline & & & $P 6 / m(h)$ \\
\hline & & & $P \overline{3} 1 m(h)$ \\
\hline & & 3.. & $P 6 / m e c(h)$ \\
\hline & & & ${ }^{P 6_{3}} / \mathrm{mem}(h)$ \\
\hline \multirow[t]{4}{*}{$\mathrm{E} 2 \mathrm{z}$} & \multirow[t]{4}{*}{4} & $3 m$. & ${ }^{P 6_{3}} / \mathrm{mme}(f)$ \\
\hline & & 3.. & $P \overline{6} 2 c(f)$ \\
\hline & & & $P 6_{3} 22(f)$ \\
\hline & & & $\begin{array}{l}P 6_{3} / m(f) \\
P \overline{3} 1 c(f)\end{array}$ \\
\hline \multirow[t]{3}{*}{$\mathrm{R} 2 \mathrm{z}$} & \multirow[t]{3}{*}{6} & $3 m$. & $R \overline{3} m$ (c) \\
\hline & & 3.. & $R 32(c)$ \\
\hline & & & $R \overline{3} \quad(c)$ \\
\hline$\left.{ }^{c}{ }^{c}\right) \cdot(R 2 z)_{c}$ & 12 & 3.. & $R 3_{c}$ (c) \\
\hline
\end{tabular}

$\left({ }^{a}\right) 100 / 010 / 002$. ( $\left.{ }^{c}\right) 0 \overline{1} 0 / 110 / 002$. ( $\left.{ }^{\mathrm{d}}\right) 100 / 010 / 00 \overline{1}$. 


\begin{tabular}{|c|c|c|c|}
\hline$\left(^{a}\right)(N 2 z)_{C}$ & 6 & $\begin{array}{l}2 m m \\
2 . \\
2 .\end{array}$ & $\begin{array}{l}P 6 / \operatorname{mmm}(i) \\
P 622(i) \\
P 6 / m(i) \\
P 6 / \operatorname{mcc}(i)\end{array}$ \\
\hline $\begin{array}{l}\mathrm{P} 6 \mathrm{x}[\mathrm{z}] \\
(\mathrm{a})(\mathrm{P} 6 \mathrm{x})_{\mathrm{C}}\end{array}$ & $\begin{array}{r}6 \\
12\end{array}$ & $\begin{array}{l}m 2 m \\
.2 . \\
\ldots m \\
.2 .\end{array}$ & $\begin{array}{l}P 6 / m m m(j),(k) 00 \frac{1}{2} \\
P 622(j),(k) 00 \frac{1}{2} \\
P \overline{3} m 1(g),(h) 00 \frac{1}{2} \\
P 6 m m(d) \\
P 6 / m c c(j) 00 \frac{1}{4} \\
P 6_{3} / m m c(i)\end{array}$ \\
\hline $\begin{array}{l}P 6 x \bar{x}[z] \\
(\mathrm{a})(\mathrm{P} 6 x \bar{x})_{C}\end{array}$ & $\begin{array}{r}6 \\
12\end{array}$ & $\begin{array}{l}m m^{2} \\
\ldots 2 \\
. m . \\
\ldots 2\end{array}$ & $\begin{array}{l}P 6 / m m m(l),(m) 00 \frac{1}{2} \\
P 622(l),(m) 00 \frac{1}{2} \\
P \overline{3} 1 m(i),(j) 00 \frac{1}{2} \\
P 6 m m(e) \\
P 6 / m c c(k) 00 \frac{1}{4} \\
P 6_{3} / m c m(i)\end{array}$ \\
\hline$\ldots 2 P c^{3 x[z]}$ & 6 & $\begin{array}{l}m 2 m \\
.2 . \\
\ldots m\end{array}$ & $\begin{array}{l}P 6_{3} / \mathrm{mcm}(g) 00 \frac{1}{4} \\
P 6_{3} 22(g) \\
P \overline{3 c} 1(f) 00 \frac{1}{4} \\
P 6_{3} \mathrm{~cm}(\mathrm{c})\end{array}$ \\
\hline .2. $P_{c} E 3 x \bar{x}[z]$ & 6 & $\begin{array}{l}m m 2 \\
\ldots 2 \\
. m\end{array}$ & $\begin{array}{l}P 6_{3} / m m c(h) 00 \frac{1}{4} \\
P 6_{3} 22(h) 00 \frac{1}{4} \\
P \overline{3} 1 c(h) 00 \frac{1}{4} \\
P 6_{3} m e(c)\end{array}$ \\
\hline$\frac{{ }^{3_{1} \cdot 2 P_{C_{c}}{ }^{+} Q_{c} 1 x}}{\text { (d) } 3_{2} \cdot 2 P_{C_{c}}{ }^{-} Q_{c} 1 x}$ & $\begin{array}{l}6 \\
6\end{array}$ & $\begin{array}{l}.2 . \\
.2\end{array}$ & $\begin{array}{l}P 6_{1} 22(a) \\
P 6_{5} 22(a)\end{array}$ \\
\hline 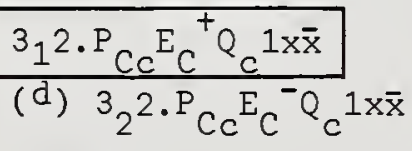 & $\begin{array}{l}6 \\
6\end{array}$ & $\begin{array}{l}\ldots 2 \\
\ldots 2\end{array}$ & $\begin{array}{l}P 6_{1} 22(b) 00 \frac{11}{12} \\
P 6_{5} 22(b) 00 \frac{1}{12}\end{array}$ \\
\hline$\frac{{ }^{+} \mathrm{Q} 2 \mathrm{z}}{(\mathrm{d}){ }_{\mathrm{Q} 2 \mathrm{z}}}$ & $\begin{array}{l}6 \\
6\end{array}$ & $\begin{array}{l}2 . . \\
2 . .\end{array}$ & $\begin{array}{l}P 6_{2} 22(f) \\
P 6_{4} 22(f)\end{array}$ \\
\hline
\end{tabular}

(a) $100 / 010 / 002$. (d) $100 / 010 / 00 \overline{1}$. 


\begin{tabular}{|c|c|c|c|}
\hline 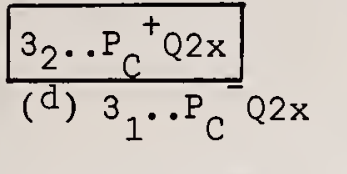 & 6 & $\begin{array}{l}.2 . \\
.2 .\end{array}$ & $\begin{array}{l}P 6_{2} 22(\mathrm{~g}),(h) 00 \frac{1}{2} \\
P 6_{4} 22(\mathrm{~g}),(h) 00 \frac{1}{2}\end{array}$ \\
\hline$\frac{{ }_{{ }_{2} \ldots P_{C}{ }^{+} Q 2 x \bar{x}}}{\text { (d) }{ }^{3}{ }_{1} \cdots P_{C} Q 2 x \bar{x}}$ & $\begin{array}{l}6 \\
6\end{array}$ & $\begin{array}{l}\ldots 2 \\
\ldots 2\end{array}$ & $\begin{array}{l}P_{2} 22(i) 00 \frac{1}{3},(j) 00 \frac{5}{6} \\
P_{4}{ }_{4}^{22(i)} 00 \frac{2}{3},(j) 00 \frac{1}{6}\end{array}$ \\
\hline$R 3 x \bar{x}[z]$ & 9 & . $m$. & $R 3 m(b)$ \\
\hline$R 3 x$ & 9 & .2. & 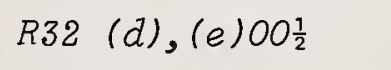 \\
\hline R6x & 18 & .2 & $R \overline{3} m(f),(g) 00 \frac{1}{2}$ \\
\hline .n.' $R_{c} 3 x$ & 18 & .2 & $R \overline{3} c(e) 00 \frac{1}{4}$ \\
\hline
\end{tabular}

Hexagonal and rhombohedral, bivariant

\begin{tabular}{|c|c|c|c|}
\hline$\frac{\text { P3xy }}{\text { P3xy }[z]}$ & $\begin{array}{l}3 \\
3\end{array}$ & $\begin{array}{c}m_{0} . \\
1\end{array}$ & $\begin{array}{ll}P \overline{6} & (j),(k) 00 \frac{1}{2} \\
P 3 & (d)\end{array}$ \\
\hline$\frac{3_{2} \cdot P_{C}^{+} Q 1 x y}{\left(d^{2}\right) 3_{1} \cdots P_{C} Q 1 x y[z]}$ & 3 & 1 & $\begin{array}{l}\mathrm{P3}_{2}(\mathrm{a}) \\
\mathrm{P3} 3_{1}(a)\end{array}$ \\
\hline$\frac{\mathrm{P} 3 \mathrm{x} \overline{\mathrm{x}} 2 \mathrm{y}}{\mathrm{P} 3 \mathrm{x} \overline{\mathrm{x}} 2 \mathrm{y}[\mathrm{z}]}$ & 6 & $\begin{array}{l}m_{0} . \\
1\end{array}$ & $\begin{array}{l}P \bar{\sigma} m 2(\mathrm{l}),(m) 00 \frac{1}{2} \\
P 3 m 1(e)\end{array}$ \\
\hline $\mathrm{P} 3 \mathrm{x} \bar{x} 2 \mathrm{z}$ & 6 &.$m$. & $P \overline{6} m 2(n)$ \\
\hline$\frac{\cdots^{2 P} c^{3 x y}}{. .2 P 3 x y[z]}$ & 6 & $\begin{array}{l}m . \cdot \\
1\end{array}$ & $\begin{array}{l}P \bar{C} c 2(k) 00 \frac{1}{4} \\
P 3 c 1(d)\end{array}$ \\
\hline P3x2z & 6 & .. m & $P \bar{\sigma} 2 m(i)$ \\
\hline$\frac{\text { P3x2y }}{\text { P3x2y }[z]}$ & $\begin{array}{l}6 \\
6\end{array}$ & $\begin{array}{l}m_{0} . \\
1\end{array}$ & $\begin{array}{l}P \overline{6} 2 m(j),(k) 00 \frac{1}{2} \\
P 31 m(d)\end{array}$ \\
\hline
\end{tabular}

(d) $100 / 010 / 00 \overline{1}$. 


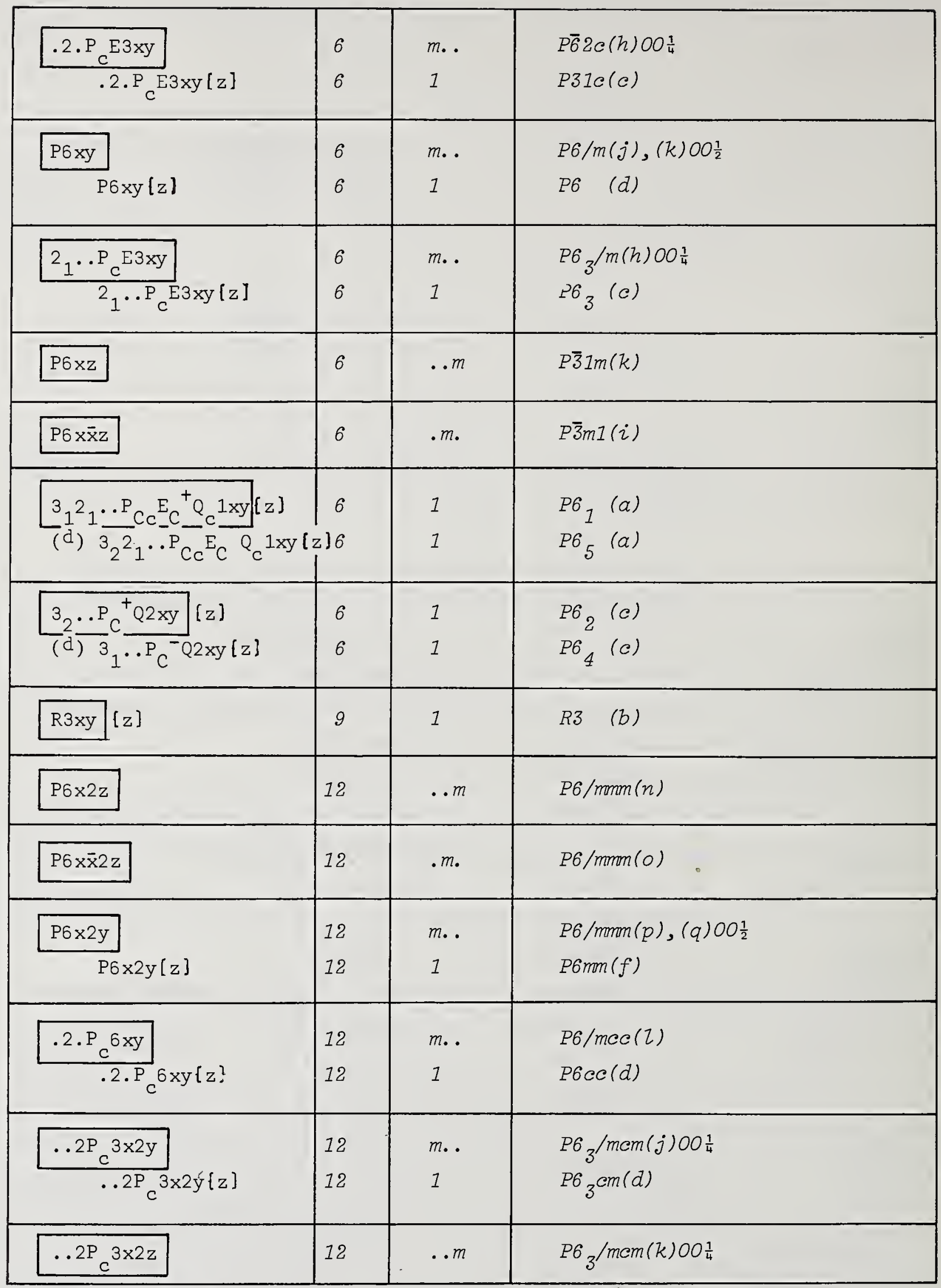

( $\left.{ }^{\mathrm{d}}\right) 100 / 010 / 00 \overline{1}$. 


\begin{tabular}{|c|c|c|c|}
\hline$\frac{\cdot 2 \cdot P_{c}^{E 3 x \bar{x} 2 y}}{.2 \cdot P_{c} E 3 x \bar{x} 2 y[z]}$ & $\begin{array}{l}12 \\
12\end{array}$ & $\begin{array}{l}m_{.} . \\
1\end{array}$ & $\begin{array}{l}P 6_{3} / m m c(j) 00 \frac{1}{4} \\
P 6_{3} m c(d)\end{array}$ \\
\hline -2. ${ }_{C} E 3 x \times \bar{x} 2 z$ & 12 &.$m$. & $P 6_{3} / m m c(k) 00 \frac{1}{4}$ \\
\hline $\mathrm{R} 6 \mathrm{x} \overline{\mathrm{x}} \mathrm{z}$ & 18 &.$m$. & $R \overline{3} m(h)$ \\
\hline$R 3 x \bar{x} 2 y[z]$ & 18 & 1 & $R 3 m(c)$ \\
\hline$\cdot n \cdot{ }^{\prime} R_{c} 3 x y \mid\{z\}$ & 18 & 1 & $R 3 c(b)$ \\
\hline
\end{tabular}

Hexagonal and rhombohedral, trivariant (site symmetry 1)

The 6-pointers:

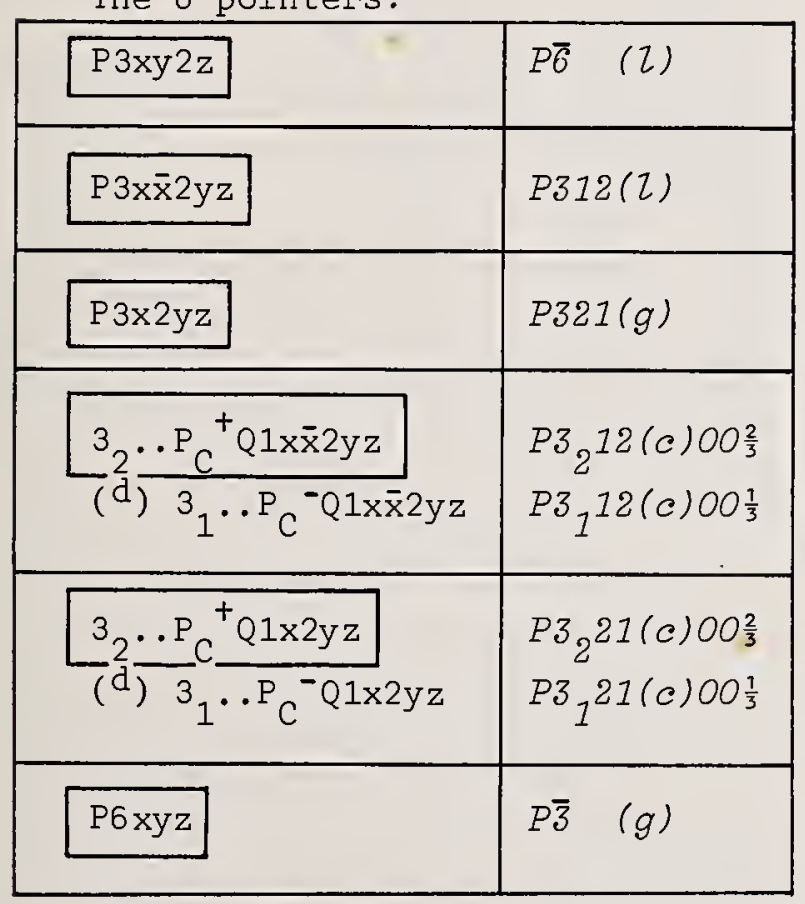

\begin{tabular}{|c|c|}
\hline .2. $\mathrm{P}_{c^{\mathrm{E}}} \mathrm{xy} 2 \mathrm{z}$ & $P \overline{6} 2 c(i) 00 \frac{1}{4}$ \\
\hline P6x2yz & $P 622(n)$ \\
\hline$\frac{3}{d} \frac{1.2 P_{C c}{ }^{+} Q_{c} 1 \times 2 y z}{3_{2} \cdot 2 P_{C c}{ }^{-} Q_{c} 1 \times 2 y z}$ & $\begin{array}{l}P 6_{1} 22(c) \\
P 6_{5} 22(c)\end{array}$ \\
\hline 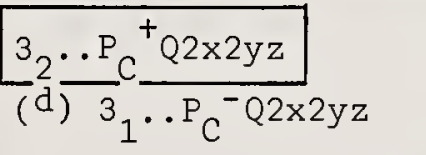 & $\begin{array}{l}P 6{ }_{2} 22(k) \\
P 6_{4} 22(k)\end{array}$ \\
\hline$\cdots 2{ }_{c} 3 \times 2 y z$ & $P 6_{3} 22(i)$ \\
\hline P6xy 2z & $P 6 / m(Z)$ \\
\hline${ }_{1} \ldots P_{C}$ E3xy $2 z$ & $P 6_{3} / m(i) 00 \frac{1}{4}$ \\
\hline P6xz2y & $P \overline{3} 1 m(乙)$ \\
\hline$\ldots c P_{c} E 3 x \bar{x} 2 y z$ & $P \overline{3} 1 c(i) 00 \frac{1}{4}$ \\
\hline
\end{tabular}

The 12-pointers:

\begin{tabular}{|l|l|}
\hline $\mathrm{P} 3 \mathrm{x} \overline{\mathrm{x}} 2 \mathrm{y} 2 \mathrm{z}$ & $P \overline{6} \mathrm{~m} 2(0)$ \\
\hline $\mathrm{P} 2 \mathrm{P} \mathrm{c}^{3 \mathrm{xy} 2 \mathrm{z}}$ & $P \overline{6} c 2(\mathrm{z}) 00 \frac{1}{4}$ \\
\hline $\mathrm{P} 3 \mathrm{x} 2 \mathrm{y} 2 \mathrm{z}$ & $P \overline{6} 2 m(\mathrm{Z})$ \\
\hline
\end{tabular}

(d) $100 / 010 / 00 \overline{1}$. 


\begin{tabular}{|c|c|}
\hline P6x $x \bar{x} z 2 y$ & $P \overline{3} m 1(j)$ \\
\hline . n. $P_{c} 3 x 2 y z$ & $P \overline{3} c 1(g) 00 \frac{1}{4}$ \\
\hline
\end{tabular}

The 18-pointers:

\begin{tabular}{|l|l|}
\hline$R 3 \times 2 y z$ & $R 32(f)$ \\
\hline$R 6 x y z$ & $R \overline{3}(f)$ \\
\hline
\end{tabular}

The 24-pointers:

\begin{tabular}{|c|c|}
\hline$P 6 x 2 y 2 z$ & $P 6 /$ mmon $(r)$ \\
\hline$m . P_{c} 6 x 2 y z$ & $P 6 /$ mec $(m) 00 \frac{1}{4}$ \\
\hline$\ldots 2 \mathrm{P}_{c}^{3 \times 2 y 2 z}$ & $P 6_{3} / \operatorname{mem}(2) 00 \frac{1}{4}$ \\
\hline .2. $P_{c} E 3 x \bar{x} 2 y 2 z$ & $P 6_{3} /$ mme (2) $00 \frac{1}{4}$ \\
\hline
\end{tabular}

The 36-pointers:

\begin{tabular}{|l|l|}
\hline R6xīz2y & $R \overline{3} m(i)$ \\
\hline. $\mathrm{n} \cdot{ }^{\prime} \mathrm{R}_{\mathrm{c}} 3 \mathrm{x} 2 \mathrm{yz}$ & $R \overline{3} c(f) 00 \frac{\mathrm{T}}{4}$ \\
\hline
\end{tabular}


Table 28

\begin{tabular}{|l|l|l|l|}
\hline $\begin{array}{l}\text { Lattice complex } \\
\text { in all its } \\
\text { representations }\end{array}$ & Multi- & $\begin{array}{l}\text { Site } \\
\text { symmetry } \\
\text { (oriented) }\end{array}$ & $\begin{array}{l}\text { Occurrence: } \\
\text { space group, Wyckhoff letter, } \\
\text { shift from standard representation }\end{array}$ \\
\hline
\end{tabular}

Tetragonal, invariant

\begin{tabular}{|c|c|c|c|c|}
\hline \multirow[t]{8}{*}{$\mathrm{P}$} & & 1 & $4 / \mathrm{mmm}$ & $P 4 / \mathrm{mmm}(a),(b) 00 \frac{1}{2},(c) \frac{1}{2} \frac{1}{2} O,(d) \frac{1}{2} \frac{1}{2} \frac{1}{2}$ \\
\hline & & & $\overline{4} 2 m$ & $P \overline{4} 2 m(a),(b) \frac{1}{2} \frac{1}{2} \frac{1}{2},(c) 00 \frac{1}{2},(d) \frac{1}{2} \frac{1}{2} O$ \\
\hline & & & $\overline{4} m 2$ & $P \overline{4} m 2(a),(b) \frac{1}{2} \frac{1}{2} O,(c) \frac{1}{2} \frac{1}{2} \frac{1}{2},(d) 00 \frac{1}{2}$ \\
\hline & & & 422 & $P 422(a),(b) 00 \frac{1}{2},(c) \frac{1}{2} \frac{1}{2} O$, (d) $\frac{1}{2} \frac{1}{2} \frac{1}{2}$ \\
\hline & & & $4 / m$. & $P 4 / m(a),(b) 00 \frac{1}{2},(c) \frac{1}{2} \frac{1}{2} O$, , d ) $\frac{1}{2} \frac{1}{2} \frac{1}{2}$ \\
\hline & & & $\overline{4}$. & $P \overline{4} \quad(a),(b) 00 \frac{1}{2},(c) \frac{1}{2} \frac{1}{2} O$, (d) $\frac{1}{2} \frac{1}{2} \frac{1}{2}$ \\
\hline & $P\{z\}$ & 1 & $4 m m$ & $P 4 m m(a),(b) \frac{1}{2} \frac{1}{2} O$ \\
\hline & & & 4.. & P4 (a), (b) $\frac{1}{2} \frac{1}{2} O$ \\
\hline \multirow[t]{14}{*}{$\left({ }^{a}\right)$} & $\mathrm{P}_{c}$ & 2 & $\overline{4} 2 m$ & $P 4_{2} / \mathrm{mcm}(a) 00 \frac{1}{4},(b) \frac{1}{2} \frac{1}{2} \frac{1}{4}$ \\
\hline & & & $\overline{4} m 2$ & $P 4_{2} / m m c(e) 00 \frac{1}{4},(f) \frac{1}{2} \frac{1}{2} \frac{1}{4}$ \\
\hline & & & 422 & $P 4 / \operatorname{mcc}(a) 00 \frac{1}{4},(c) \frac{1}{2} \frac{1}{2} \frac{1}{4}$ \\
\hline & & & $4 / m$. & $P 4 / \operatorname{mcc}(b),(d) \frac{1}{2} \frac{1}{2} O$ \\
\hline & & & $\overline{4} .$. & $P \overline{4} 2 c(e),(f) \frac{1}{2} \frac{1}{2} O$ \\
\hline & & & & $P \overline{4} c 2(c),(d) \frac{1}{2} \frac{1}{2} O$ \\
\hline & & & & $P 4_{2} / m(e) 00 \frac{1}{4},(f) \frac{1}{2} \frac{1}{2} \frac{1}{4}$ \\
\hline & & & mmm. & $P 4_{2} / m n c(a),(b) \frac{1}{2} \frac{1}{2} O$ \\
\hline & & & $m \cdot m m$ & $P 4_{2} / \mathrm{mem}(b),(d) \frac{1}{2} \frac{1}{2} O$ \\
\hline & & & 222. & $P \overline{4} 2 c(a) 00 \frac{1}{4},(c) \frac{1}{2} \frac{1}{2} \frac{1}{4}$ \\
\hline & & & & $P 4_{2} 22(a),(b) \frac{1}{2} \frac{1}{2} O$ \\
\hline & & & 2.22 & $P \overline{4} c 2(a) 00 \frac{1}{4},(b) \frac{1}{2} \frac{1}{2} \frac{1}{4}$ \\
\hline & & & & $P 42^{22(e) 00 \frac{1}{4},(f) \frac{1}{2} \frac{1}{2} \frac{1}{4}}$ \\
\hline & & & $2 / m_{.} \cdot$ & $P 4_{2} / m(a),(b) \frac{1}{2} \frac{1}{2} O$ \\
\hline \multirow[t]{4}{*}{$\left({ }^{a}\right)$} & $P_{c}[z]$ & 2 & & $P 4 c c(a),(b) \frac{1}{2} \frac{1}{2} O$ \\
\hline & & & $2 m m$. & $P 4_{2} m c(a),(b) \frac{1}{2} \frac{1}{2} O$ \\
\hline & & & 2. $m m$ & $P 4_{2} \mathrm{~cm}(a),(b) \frac{1}{2} \frac{1}{2} \mathrm{O}$ \\
\hline & & & 2. & $P 4_{2}(a),(b) \frac{1}{2} \frac{1}{2} O$ \\
\hline \multirow[t]{2}{*}{$\left({ }^{b}\right)$} & c & 2 & $\overline{4} 2 m$ & $P 4 / n b m(c) O \frac{1}{2} O$, (d) $O \frac{1}{2} \frac{1}{2}$ \\
\hline & & & $\overline{4} m 2$ & $P 4 / n m m(a),(b) 00 \frac{1}{2}$ \\
\hline
\end{tabular}

( $\left.{ }^{a}\right) 100 / 010 / 002$. $\left({ }^{b}\right) 110 / \overline{1} 10 / 001$. From standard representation to cell. 


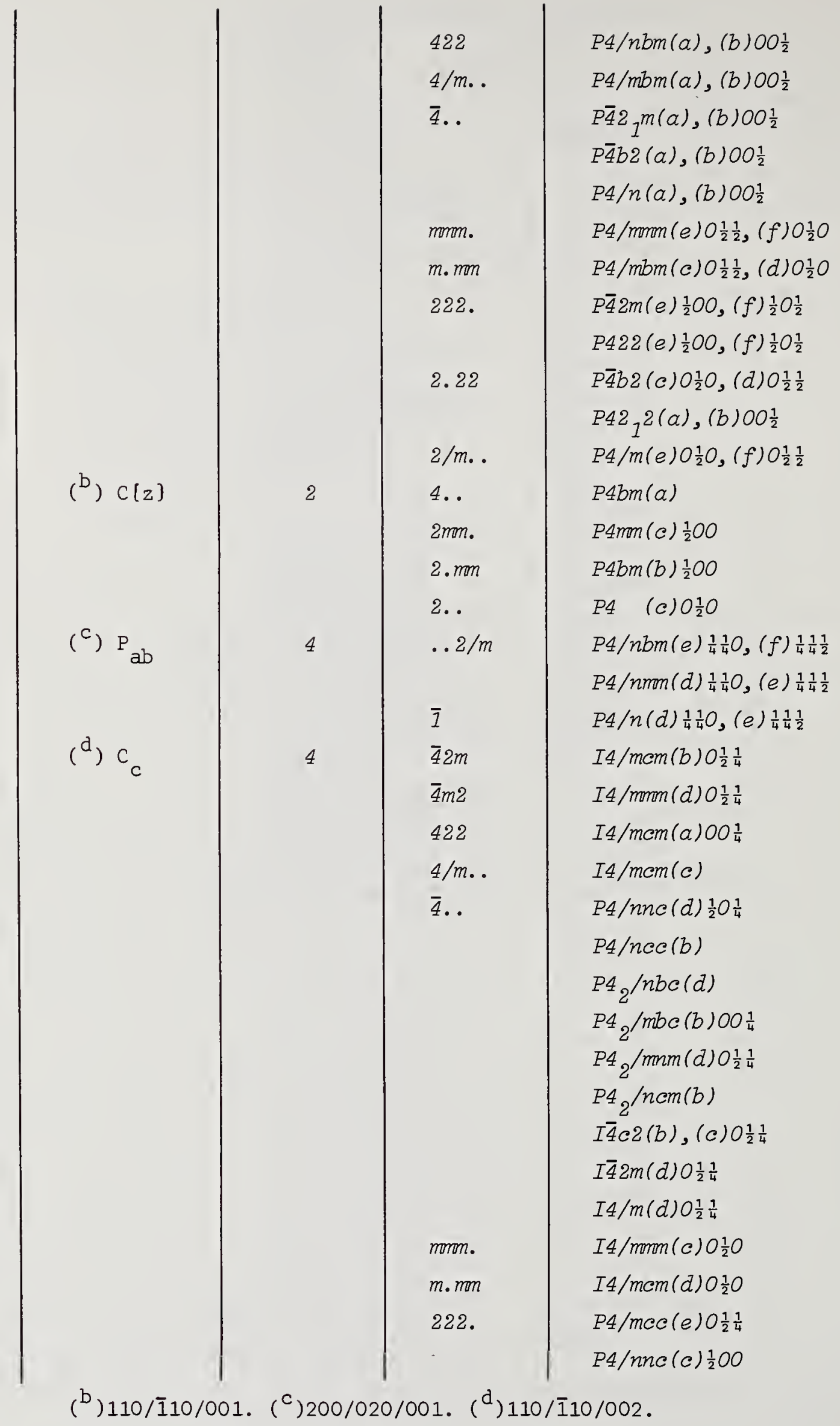




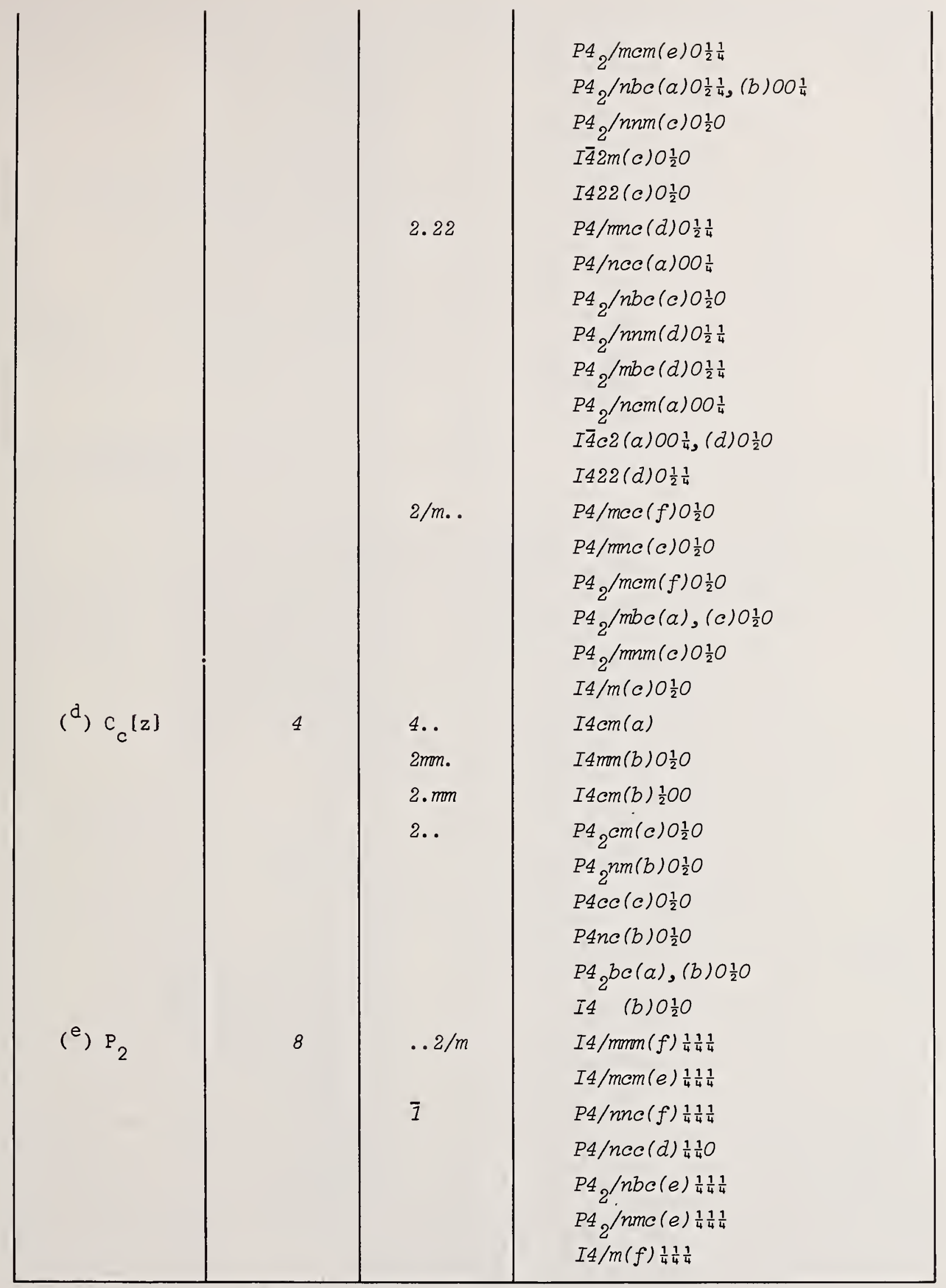

( $\left.{ }^{d}\right) 110 / \overline{1} 10 / 002$. $\left({ }^{e}\right) 200 / 020 / 002$. 


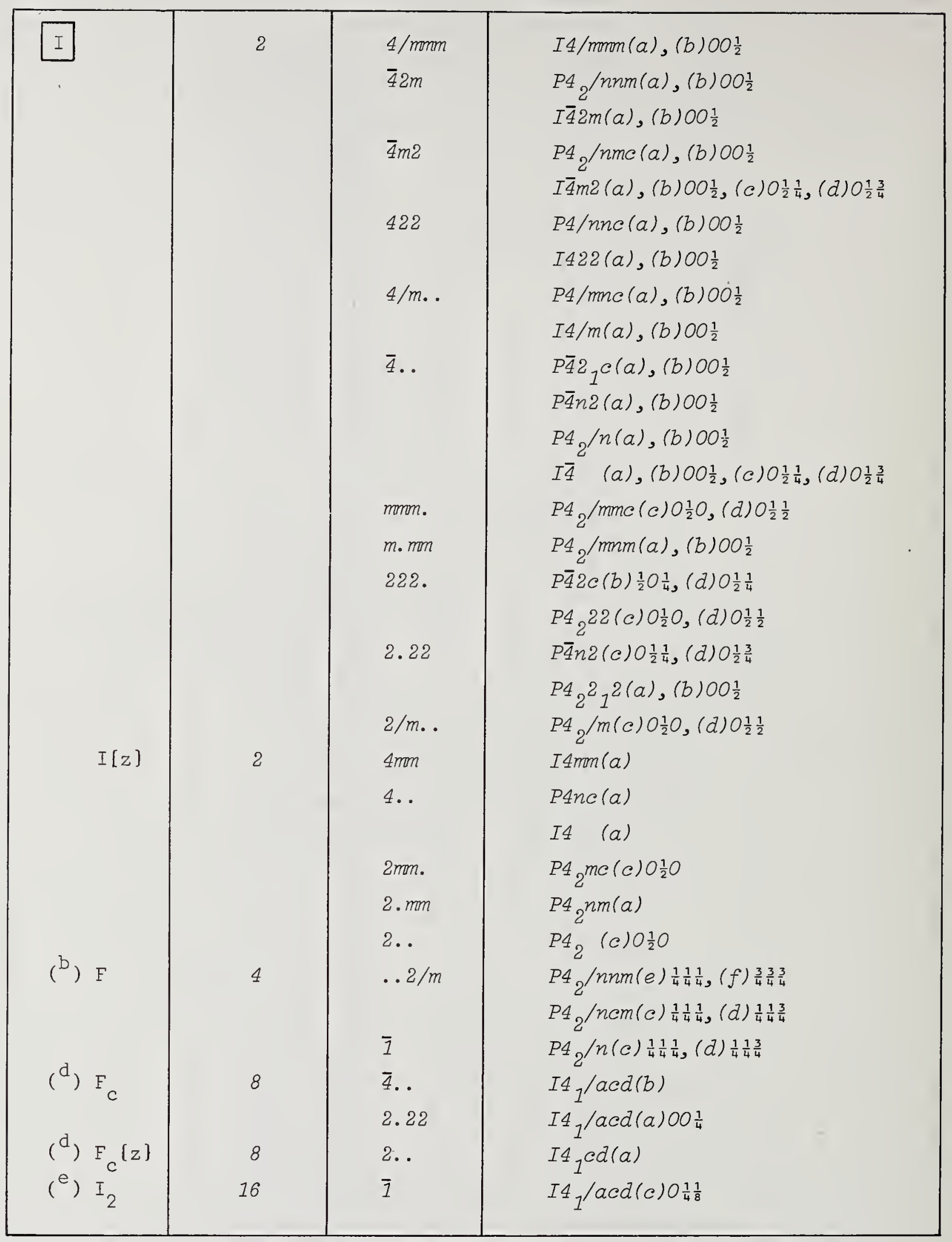

( $\left.{ }^{b}\right) 110 / \overline{1} 10 / 001 .\left({ }^{d}\right) 110 / \overline{1} 10 / 002 .\left({ }^{e}\right) 200 / 020 / 002$. 


\begin{tabular}{|c|c|c|c|}
\hline $\begin{array}{l}\frac{D}{\left({ }^{F}\right)} v_{D} \\
\left({ }^{f}\right) v_{D}[z]\end{array}$ & 4 & $\begin{array}{l}\overline{4} 2 m \\
\overline{4} m 2 \\
\overline{4} . . \\
2.22 \\
2 m m . \\
2 .\end{array}$ & $\begin{array}{l}E 4_{1} / d d m^{I} \\
I 4_{1} / a m d(a),(b) 00 \frac{1}{2} \\
I \overline{4} 2 d(a),(b) 00 \frac{1}{2} \\
I 4_{1} / a(a),(b) 00 \frac{1}{2} \\
I 4_{1} 22(a),(b) 00 \frac{1}{2} \\
I 4_{1} m a(a) \\
I 4_{1}(a)\end{array}$ \\
\hline$\frac{T}{\left({ }^{F}\right)} v_{T}$ & $\begin{array}{r}16 \\
8\end{array}$ & $\begin{array}{l}\cdots 2 / m \\
.2 / m \\
\bar{I}\end{array}$ & $\begin{array}{l}F 4_{1} / d d m^{1} \\
I 4_{1} / a m d(c),(d) 00 \frac{1}{2} \\
I_{1} / a(c),(d) 00 \frac{1}{2}\end{array}$ \\
\hline
\end{tabular}

Tetragonal, univariant

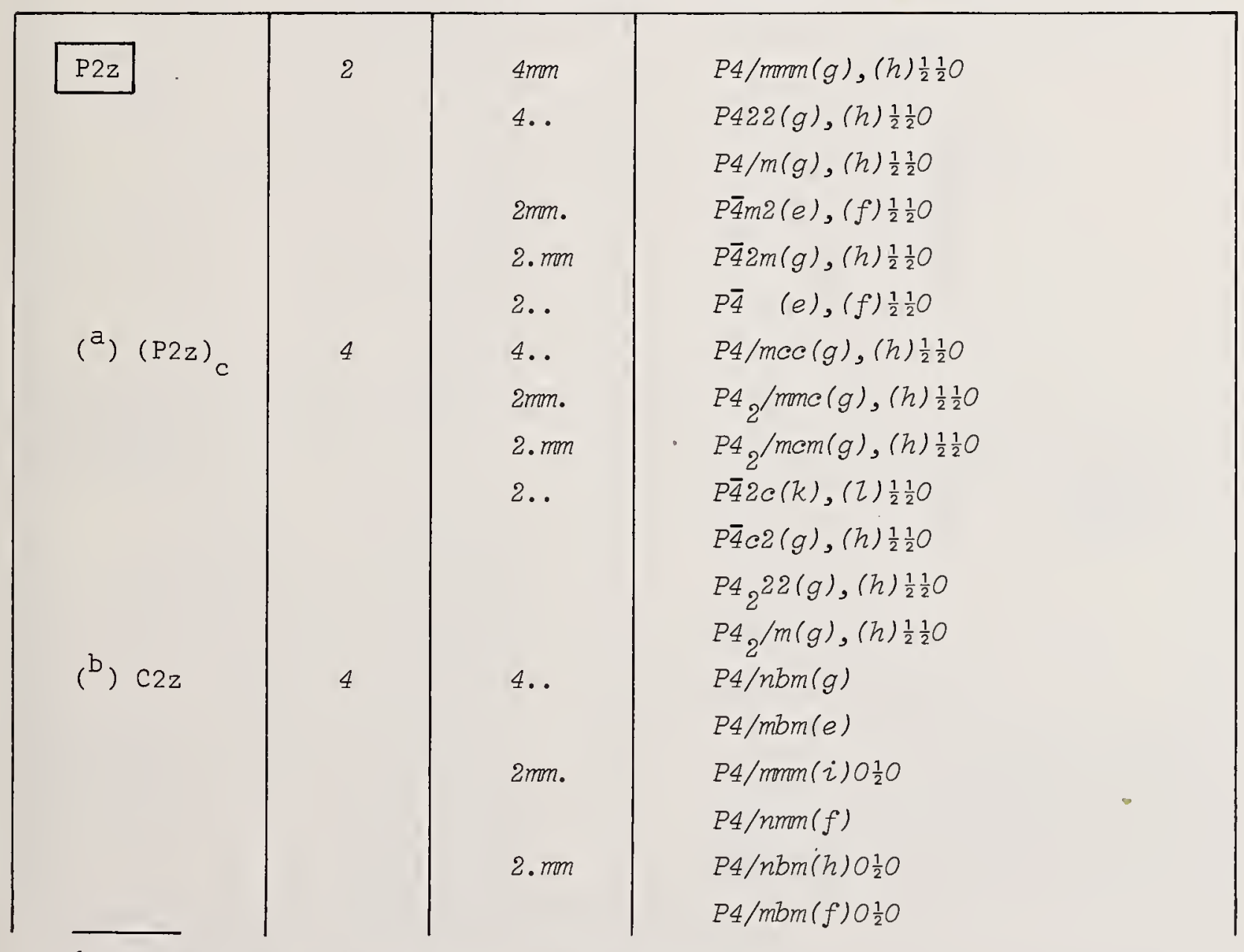

${ }^{1}$ Unconventional setting. $C_{p}$. next entry.

(a) $100 / 010 / 002$. (b) $110 / 1 \overline{1} 0$ '001. (f) $\frac{1}{2} \frac{\pi}{2} 0 / \frac{1}{2} \frac{1}{2} 0 / 001$. 


\begin{tabular}{|c|c|c|c|}
\hline$\left.{ }^{d}\right)(C 2 z)_{c}$ & . & $\begin{array}{l}4 . . \\
2 m m . \\
2 . m m \\
2 . .\end{array}$ & $\begin{array}{l}P \overline{4} 2 m(m) O \frac{1}{2} O \\
P \overline{4} 2 I_{1}(d) \\
P \overline{4} b 2(e),(f) O \frac{1}{2} O \\
P 422(i) O \frac{1}{2} O \\
P 42_{1} 2(d) \\
P 4 / m(i) O \frac{1}{2} O \\
P 4 / n(f) \\
I 4 / m c m(f) \\
I 4 / m m m(g) O \frac{1}{2} O \\
I 4 / m c m(g) O \frac{1}{2} O \\
P 4 / m c c(i) O \frac{1}{2} O \\
P 4 / n n c(g) \frac{1}{2} O O \\
P 4 / m n c(f) O \frac{1}{2} O \\
P 4 / n c c(e) \\
P 4{ }_{2} / m c m(k) O \frac{1}{2} O \\
P 4{ }_{2} / n b c(f) O \frac{1}{2} O,(g) \\
P 4{ }_{2} / n n m(h) O \frac{1}{2} O \\
P 4{ }_{2} / m b c(e),(f) O \frac{1}{2} O \\
P 44_{2} / m m(h) O \frac{1}{2} O \\
P 44_{2} / n c m(f) \\
I \overline{4} c 2(f),(g) O \frac{1}{2} O \\
I \overline{4} 2 m(h) O \frac{1}{2} O \\
I 422(f) O \frac{1}{2} O \\
I 4 / m(g) O \frac{1}{2} O\end{array}$ \\
\hline$\left({ }^{a}\right)_{0}(.2 \mathrm{CI} 1 z)_{C}$ & 4 & $\begin{array}{l}4 m m \\
4 . . \\
2 m m . \\
2 . m m \\
2 . . \\
4 . . \\
2 m m . \\
2 . m m \\
2 . .\end{array}$ & $\begin{array}{l}P 4 / n m m(c) O \frac{1}{2} O \\
P 42{ }_{1} 2(c) O \frac{1}{2} O \\
P 4 / n(c) O \frac{1}{2} O \\
\overline{4} m 2(g) O \frac{1}{2} O \\
\overline{4} 2{ }_{1} m(c) O \frac{1}{2} O \\
P \overline{4} \quad(g) O \frac{1}{2} O \\
P 4 / n c c(c) O \frac{1}{2} O \\
P 4{ }_{2} / n m c(d) O \frac{1}{2} O \\
P{ }_{2} / n c m(e) O \frac{1}{2} O \\
P \overline{4}{ }_{1} c(d) O \frac{1}{2} O\end{array}$ \\
\hline
\end{tabular}

$\left({ }^{d}\right) 110 / \overline{1} 10 / 002 .\left(^{a}\right) 100 / 010 / 002$. 


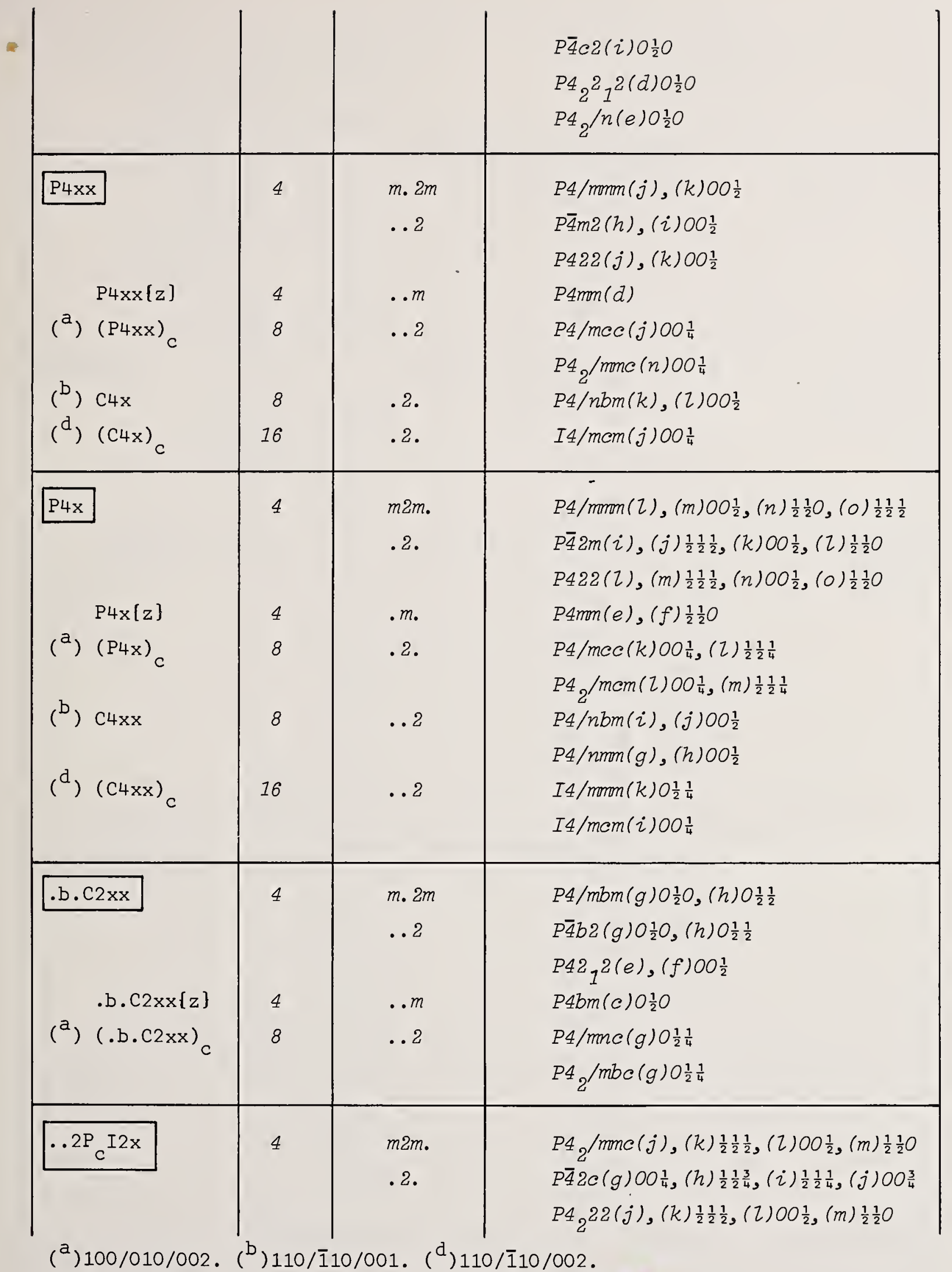




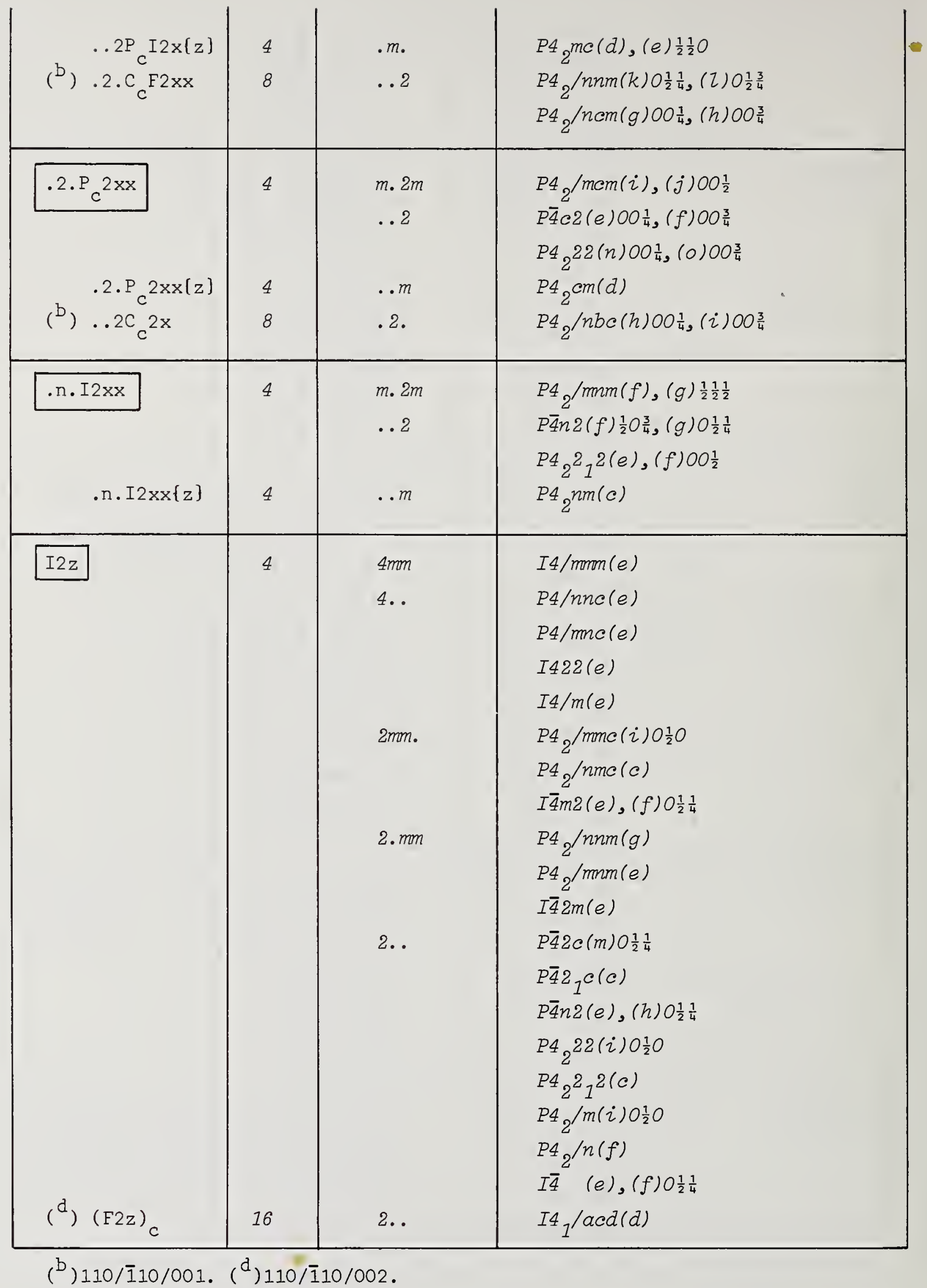




\begin{tabular}{|c|c|c|c|}
\hline$\frac{4_{3} \cdot{ }^{P}{ }_{c c}{ }^{I} c^{1 x}}{(g){ }_{1} \cdots P_{c c}{ }^{I} 1 x}$ & 4 & $\begin{array}{l}.2 \\
.2\end{array}$ & 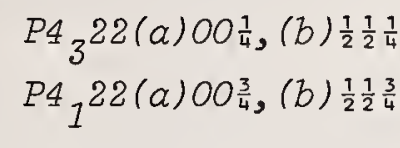 \\
\hline 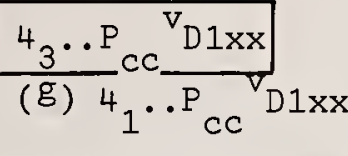 & 4 & $\begin{array}{l}\ldots 2 \\
\ldots 2\end{array}$ & $\begin{array}{l}P 4_{3} 22(c) 00 \frac{5}{8} \\
P 4_{1} 22(c) 00 \frac{3}{8}\end{array}$ \\
\hline$\frac{{ }^{4}}{3} \frac{\ldots I}{\left.g^{2}\right)}{ }^{4_{1}} \frac{{ }^{V_{D 1 x x}}}{\ldots I_{c}{ }_{D} 1 x x}$ & 4 & $\begin{array}{l}\ldots 2 \\
\ldots 2\end{array}$ & $\begin{array}{l}P 43^{2} 1^{2(a)} \\
P 41^{2} 1^{2(a)}\end{array}$ \\
\hline$I 4 x \times[\dot{z}]$ & 8 & $\begin{array}{l}m .2 m \\
\ldots 2 \\
\\
\ldots m\end{array}$ & $\begin{array}{l}I 4 / \text { mmon(h) } \\
P 4 / n n c(k) \\
P 4_{2} / n m c(f) \\
I \overline{4} m 2(g),(h) O \frac{1}{2} \frac{1}{4} \\
I 422(g) \\
I 4 m m(c)\end{array}$ \\
\hline $\operatorname{I} 4 x\{z\}$ & 8 & $\begin{array}{l}m 2 m . \\
.2 . \\
. m \text {. }\end{array}$ & $\begin{array}{l}I 4 / \text { mmm }(i),(j) \frac{1}{2} \frac{1}{2} 0 \\
P 4 / n n c(i),(j) 00 \frac{1}{2} \\
P 4{ }_{2} / n n m(i),(j) 00 \frac{1}{2} \\
I \overline{4} 2 m(f),(g) 00 \frac{1}{2} \\
I 422(h),(i) 00 \frac{1}{2} \\
I 4 m m(d)\end{array}$ \\
\hline .b. $c_{c} 2 x x\{z\}$ & 8 & $\begin{array}{l}m .2 m \\
\ldots 2 \\
\\
\ldots m\end{array}$ & $\begin{array}{l}I 4 / \mathrm{mem}(h) O \frac{1}{2} O \\
P 4 / n c c(f) 00 \frac{1}{4} \\
P 42 / \mathrm{hbc}(j) 0 \frac{1}{2} O \\
I \overline{4} c 2(e) 00 \frac{1}{4},(h) O \frac{1}{2} O \\
I 422(j) O \frac{1}{2} \frac{1}{4} \\
I 4 \mathrm{~cm}(\mathrm{c}) \frac{1}{2} 00\end{array}$ \\
\hline$v_{D 2 z}$ & 8 & $\begin{array}{l}2 m m . \\
2 . .\end{array}$ & $\begin{array}{l}\text { I4 }{ }_{1} / \text { and }(e) \\
I \overline{4} 2 d(c) \\
I 4_{1} 22(c) \\
I 4_{1} / a(e)\end{array}$ \\
\hline$\overline{4} . . \mathrm{V}_{\mathrm{TF}} 1 \mathrm{x}$ & 8 & .2 & $I \overline{4} 2 d(d)$ \\
\hline
\end{tabular}

${ }^{g}$ ) $100 / 010 / 00 \overline{1}$. 


\begin{tabular}{|c|c|c|c|}
\hline $\begin{array}{lll}\ldots \mathrm{d}^{\mathrm{v}} \mathrm{D} 2 \mathrm{x} & {[\mathrm{z}]}\end{array}$ & 8 &.$m_{0}$ & $I 4_{1} m d(b)$ \\
\hline 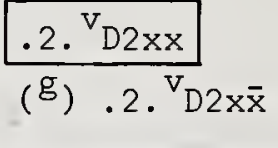 & 8 & $\begin{array}{l}\ldots 2 \\
\ldots 2\end{array}$ & $\begin{array}{l}I 4_{1} 22(d) \\
I 4_{1} 22(e)\end{array}$ \\
\hline. $.22^{\mathrm{v}_{\mathrm{TC}}} \mathrm{CC}^{1 \mathrm{x}}$ & 8 & .2 . & $I 4_{1} 22(f)$ \\
\hline$\ldots 2^{\mathrm{v}_{\mathrm{T}} 2 \mathrm{x}}$ & 16 & .2 & $I 4_{1} /$ and $(f)$ \\
\hline $\mathrm{v}_{D 4 x \mathrm{x}}$ & 16 & $\ldots 2$ & $I 4_{1} /$ amd $(g)$ \\
\hline $4 \ldots I_{2} P_{c 2} 1 x$ & 16 & .2 & $I 4_{1} / \operatorname{acd}(e) 0_{\frac{1}{4} \frac{3}{8}}$ \\
\hline .2. $F_{c} 2 x x$ & 16 &. .2 & $I 4_{1} / \operatorname{acd}(f) 00 \frac{1}{4}$ \\
\hline
\end{tabular}

Tetragonal, bivariant

\begin{tabular}{|c|c|c|c|}
\hline $\mathrm{P} 4 \mathrm{xxz}$ & 4 & ...m & $P \overline{4} 2 m(n)$ \\
\hline $.2{ }_{1} \cdot C I 1 z 2 x x$ & 4 & $\ldots m$ & $P \overline{4} 2_{1} m(e) O \frac{1}{2} O$ \\
\hline $\mathrm{P} 4 \mathrm{xz}$ & 4 &.$m_{0}$ & $P \overline{4} m 2(j),(k) \frac{1}{2} \frac{1}{2} O$ \\
\hline$\underbrace{}_{\text {P4xy }(z)}$ & $\begin{array}{l}4 \\
4\end{array}$ & $\begin{array}{l}m . . \\
1\end{array}$ & $\begin{array}{l}P 4 / m(j),(k) 00 \frac{1}{2} \\
P 4 \quad \text { (d) }\end{array}$ \\
\hline$\frac{\overline{4}^{1} \ldots P^{I 2 x y}}{\overline{4}^{1} c_{c^{I}}{ }^{I 2 x y}\{z\}}$ & 4 & $\begin{array}{l}m_{0} . \\
1\end{array}$ & $\begin{array}{l}P 4_{2} / m(j) \\
{ }^{P 4_{2}}(d)\end{array}$ \\
\hline 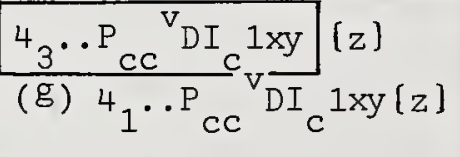 & $\begin{array}{l}4 \\
4\end{array}$ & $\begin{array}{l}1 \\
1\end{array}$ & $\begin{array}{l}P 4_{3}(a) \\
P 4_{1}(a)\end{array}$ \\
\hline$\frac{P 4 x 2 y}{P 4 x 2 y[z]}$ & $\begin{array}{l}8 \\
8\end{array}$ & $\begin{array}{l}m_{0} . \\
1\end{array}$ & $\begin{array}{l}P 4 / \mathrm{mmm}(p),(q) 00 \frac{1}{2} \\
P 4 \mathrm{~mm}(\mathrm{~g})\end{array}$ \\
\hline
\end{tabular}

(g) $100 / 010 / 00 \overline{1}$. 


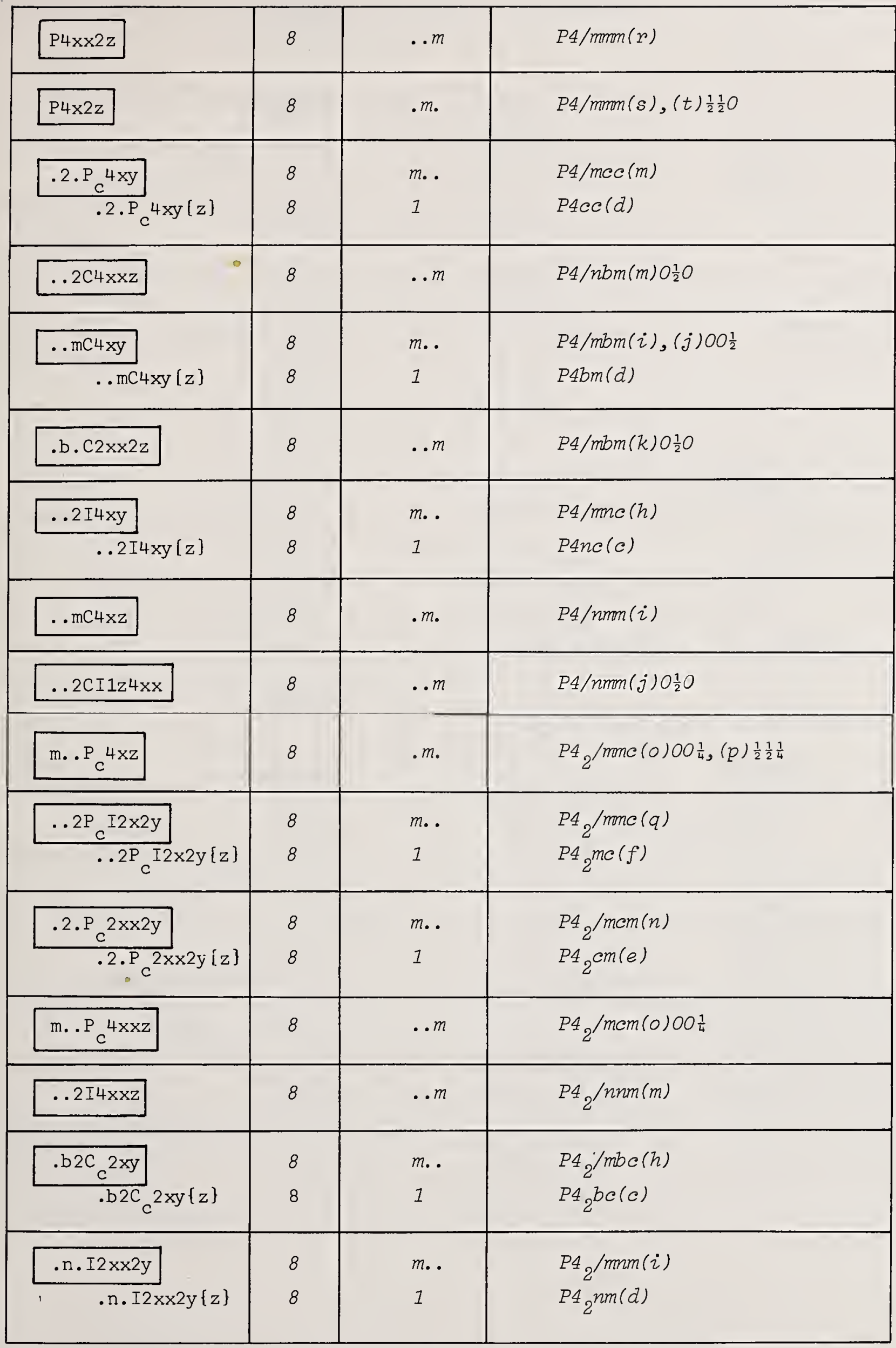




\begin{tabular}{|c|c|c|c|}
\hline .n. $12 x \times 2 z$ & 8 & ..m & ${ }^{P 4_{2}}{ }^{/ m m m}(j)$ \\
\hline .c cI $4 x z$ & 8 & . $m$ & $P 4_{2} / n m c(g)$ \\
\hline$\overline{4} . . F 2 x \times z$ & 8 & $\ldots m$ & $P 4_{2} / \operatorname{ncm}(i) \frac{1}{4} \frac{3}{4} \frac{1}{4}$ \\
\hline I $4 x z$ & 8 & $\cdot m$ & $I \overline{4} m 2(i)$ \\
\hline I $4 x \times z$ & 8 & $\ldots m$ & $I \overline{4} 2 m(i)$ \\
\hline I $4 x y$ & 8 & $m .$. & $I 4 / m(h)$ \\
\hline I $4 x y[z]$ & 8 & 1 & I4 (c) \\
\hline${ }_{4}{ }^{1} \ldots \mathrm{V}^{\mathrm{D} 2 \mathrm{xy}}[\mathrm{z}]$ & 8 & 1 & $I 4_{1}(b)$ \\
\hline$I 4 x 2 y$ & 16 & $m$. & $I 4 /$ morm (Z) \\
\hline $14 x 2 y[z]$ & 16 & 1 & $\operatorname{I4mm}(\mathrm{e})$ \\
\hline$I 4 x \times 2 z$ & 16 & $\ldots m$ & $I 4 / m m m(m)$ \\
\hline$I 4 \times 2 z$ & 16 & . $m$ & $I 4 / m m m(n)$ \\
\hline$\ldots m C_{c} 4 x y$ & 16 & $m .$. & $I 4 / m e m(k)$ \\
\hline$\ldots \mathrm{mC}_{c} 4 x y[z]$ & 16 & 1 & $I 4 \mathrm{~cm}(d)$ \\
\hline .b.c $4 x \times z$ & 16 & $\ldots m$ & $I 4 / \mathrm{mem}(Z) O \frac{1}{2} \frac{1}{4}$ \\
\hline $.2 .^{v_{D}} \times \mathrm{xz}$ & 16 &.$m$ & $I_{1} / \operatorname{lama}(h)$ \\
\hline$\ldots d^{\mathrm{V}} D 2 x 2 y$ & 16 & 1 & $I_{1} m d(c)$ \\
\hline.$b d F_{c} 2 x y[z]$ & 16 & 1 & $I 4_{1} c d(b)$ \\
\hline
\end{tabular}


The 4-pointers:

\begin{tabular}{|l|l|}
\hline P4xyz & $P \overline{4}(h)$ \\
\hline
\end{tabular}

The 8-pointers:

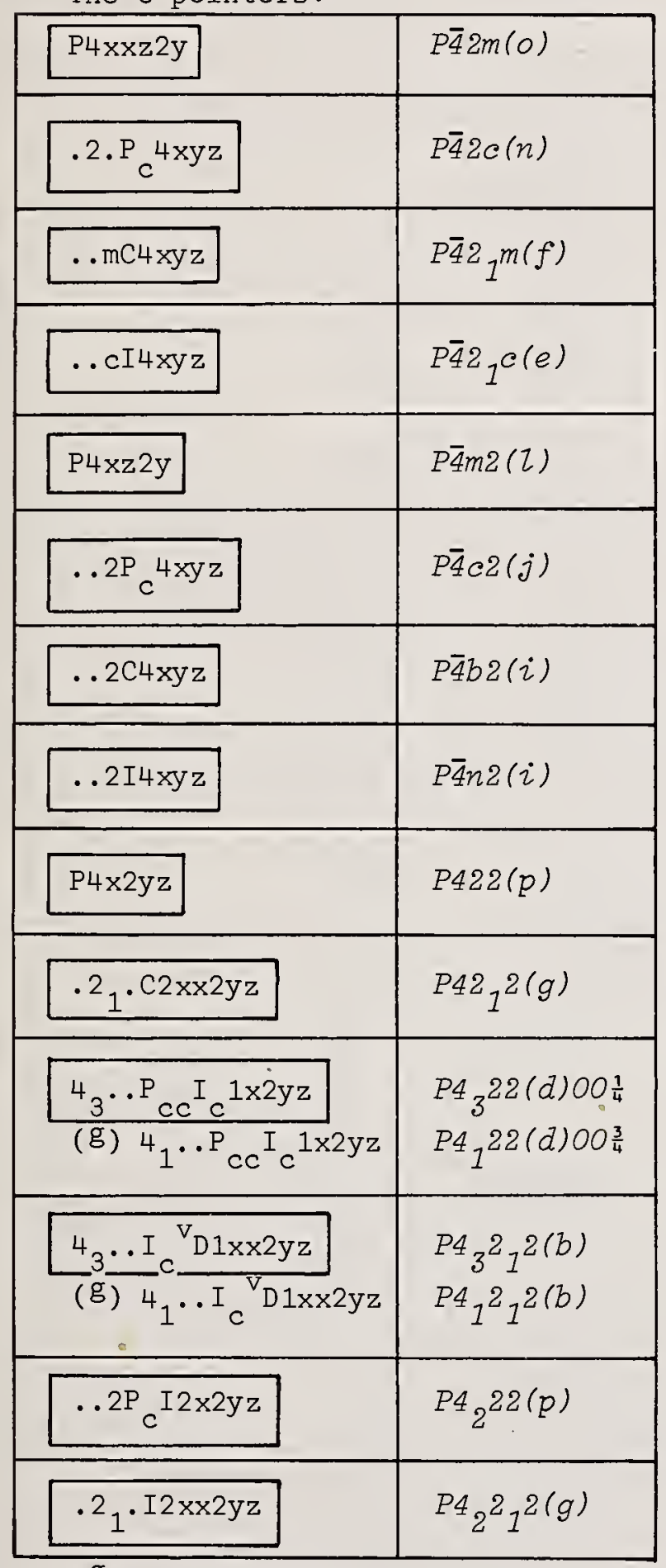

(g) $100 / 010 / 00 \overline{1}$.

\begin{tabular}{|l|l|}
\hline P4xy $2 z$ & $P 4 / m(\tau)$ \\
\hline $\mathrm{m} . \mathrm{P}_{\mathrm{c}} \mathrm{xxyz}$ & $P_{2} / \mathrm{m}(k) 00 \frac{1}{4}$ \\
\hline$\overline{1} \mathrm{C} 4 \mathrm{xyz}$ & $P 4 / n(g)$ \\
\hline $\mathrm{n} . \mathrm{I} 4 \mathrm{xyz}$ & $P_{4} / n(g)$ \\
\hline $\mathrm{I} 4 \mathrm{xyz}$ & $I \overline{4}(g)$ \\
\hline
\end{tabular}

The" 16-pointers:

\begin{tabular}{|c|c|}
\hline$P 4 x 2 y 2 z$ & $P 4 /$ morm $(u)$ \\
\hline$m . P_{c} 4 x 2 y z$ & $P 4 / \operatorname{mec}(n) 00 \frac{1}{4}$ \\
\hline$\ldots \mathrm{mC} 4 \times 2 \mathrm{yz}$ & $P 4 / n b m(n)$ \\
\hline ..cI4x $2 \mathrm{yz}$ & $P 4 / n n c(k)$ \\
\hline$\ldots \mathrm{mC} 4 \mathrm{xy} 2 \mathrm{z}$ & $P 4 / \mathrm{mbm}(\mathrm{Z})$ \\
\hline ..2I $4 x y 2 z$ & $P 4 / m c(i)$ \\
\hline$\ldots \mathrm{mC} 4 \mathrm{xz} 2 \mathrm{y}$ & $P 4 / n m m(k)$ \\
\hline.$c 2 c_{c} 4 x y z$ & $P 4 / n c e(g)$ \\
\hline m.. $P_{c} 4 x z 2 y$ & $P 4_{2} / m m c(r) 00 \frac{1}{4}$ \\
\hline m. P $P_{c}^{4 x x z 2 y}$ & $\mathrm{P}_{2}{ }_{2} / \mathrm{mcm}(p) 00 \frac{1}{4}$ \\
\hline $.22 c_{c} 4 x y z$ & $P 4_{2} / n b c(k)$ \\
\hline
\end{tabular}




\begin{tabular}{|c|c|}
\hline .. 2I $4 x x z 2 y$ & $P 4_{2} / n n m(n)$ \\
\hline$m \cdot 2 c_{c} 4 x y z$ & $P_{2} / m b c(i) 00 \frac{1}{4}$ \\
\hline .n. I $2 x \times 2 y 2 z$ & $P 4_{2} / m m(k)$ \\
\hline ..cI4xz2y & $P 4_{2} / n m c(h)$ \\
\hline$\cdots m 2 c_{c} 4 x y z$ & $P 4_{2} / n c m(j)$ \\
\hline$I 4 x z 2 y$ & $I \overline{4} m 2(j)$ \\
\hline $.2 C_{c} 4 x y z$ & $I \overline{4} c 2(i)$ \\
\hline$I 4 x x z 2 y$ & $I \overline{4} 2 m(j)$ \\
\hline .2. ${ }^{\mathrm{D}} \mathrm{D} 4 \mathrm{xyz}$ & $I \overline{4} 2 d(e)$ \\
\hline I $4 x 2 y z$ & $I 422(\mathrm{k})$ \\
\hline .2. ${ }^{\mathrm{V}} \mathrm{D} 2 \mathrm{xx} 2 \mathrm{yz}$ & $I 4_{1} 22(g)$ \\
\hline$I 4 x y 2 z$ & $I 4 / m(i)$ \\
\hline a. ${ }^{\mathrm{V}} \mathrm{D} 4 \mathrm{xyz}$ & $I 4_{1} / a(f)$ \\
\hline
\end{tabular}

The 32-pointers:

\begin{tabular}{|c|c|}
\hline I4x2y2z & $I 4 / \mathrm{mmm}(\mathrm{O})$ \\
\hline .b. $c_{c} 4 x 2 y z$ & $I 4 / \mathrm{mcm}(\mathrm{m}) 00 \frac{1}{4}$ \\
\hline .2. ${ }^{\mathrm{V}} \mathrm{D} 4 \mathrm{xz} 2 \mathrm{y}$ & $I 4_{1} / \operatorname{amd}(i)$ \\
\hline $.22 F_{c} 4 x y z$ & $I 4_{1} / \operatorname{acd}(g)$ \\
\hline
\end{tabular}


Occurrence of Lattice Complexes

Table 29

\begin{tabular}{|l|l|l|l|l|}
\hline Lattice & Transfor- & Multi- & Site & Occurrence: \\
complex & mation from & plicity & symmetry \\
in all its & standard \\
represen- & representa- \\
tations & $\begin{array}{l}\text { tion to } \\
\text { cell }\end{array}$ & & & \\
shift from standard representation & & \\
\hline
\end{tabular}

Orthorhombic, invariant

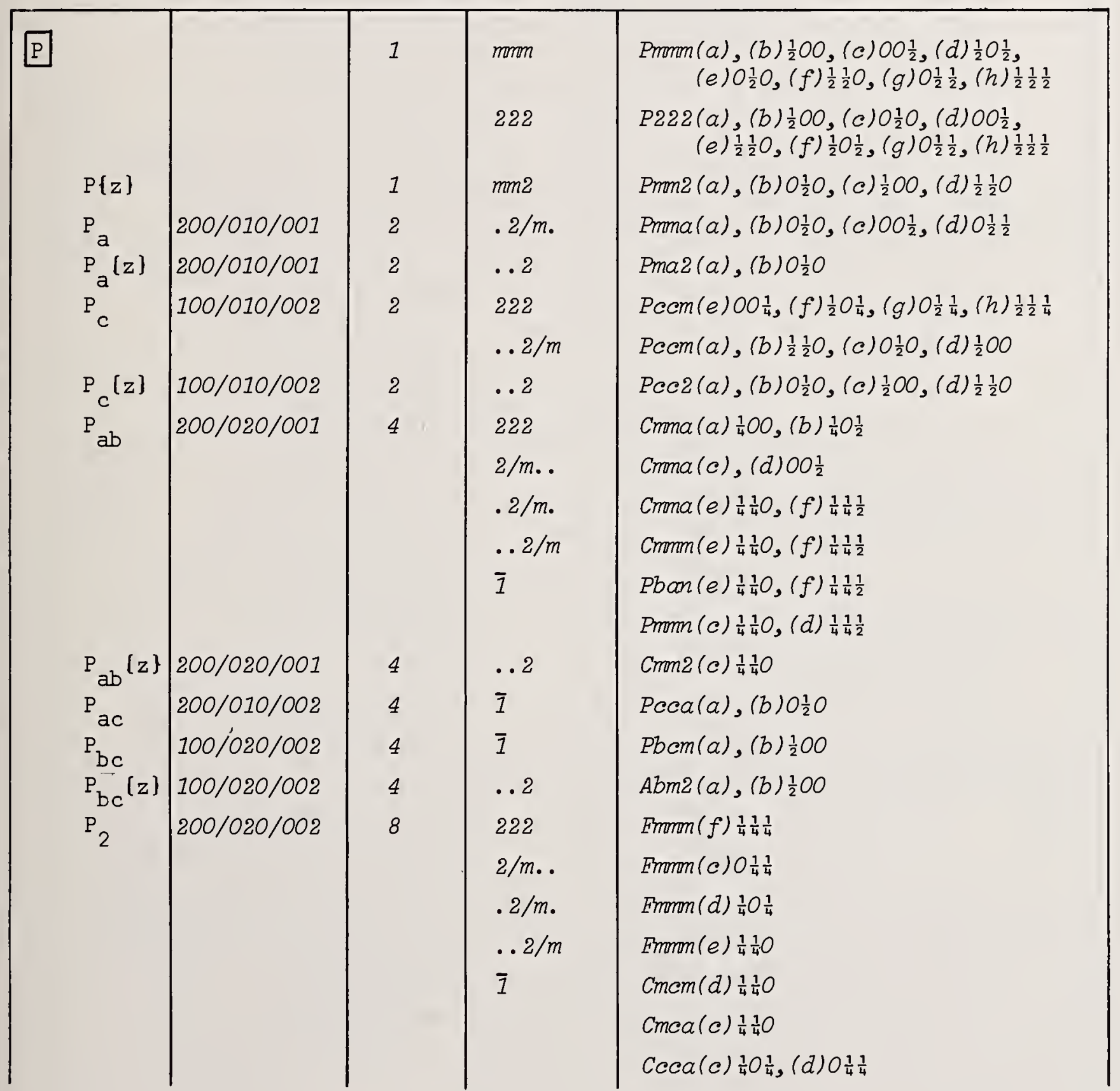




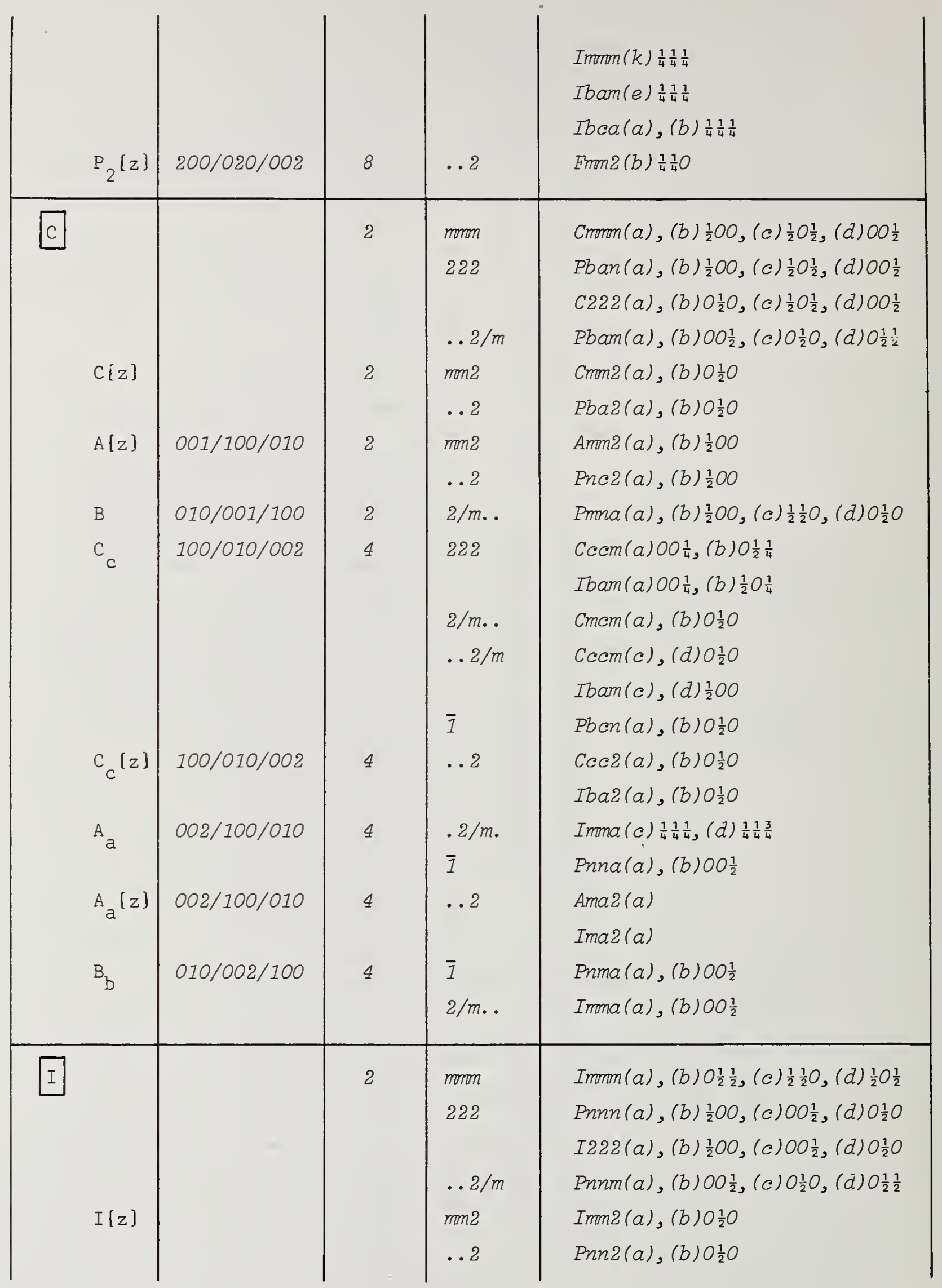




\begin{tabular}{|c|c|c|c|}
\hline $\mathrm{F}$ & 4 & $\begin{array}{l}m m m \\
222 \\
2 / m . \\
\ldots 2 / m \\
\overline{1} \\
m m 2 \\
\ldots 2\end{array}$ & 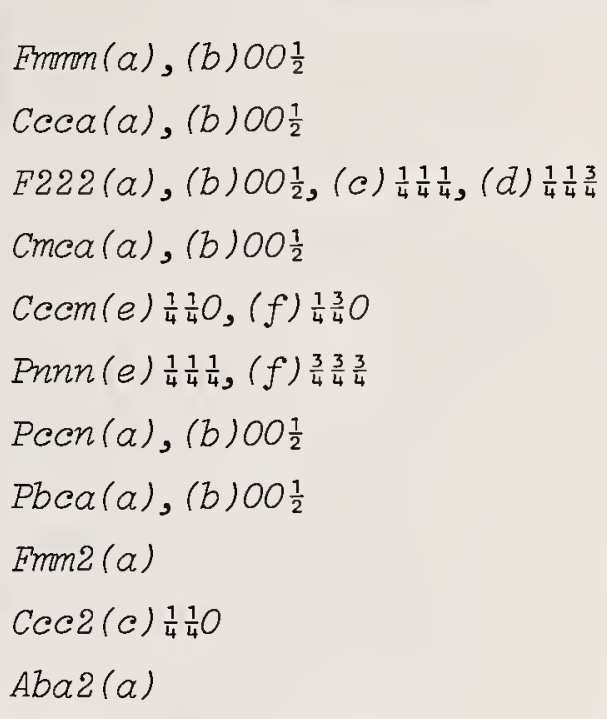 \\
\hline \begin{tabular}{|l}
$D$ \\
$D(z)$
\end{tabular} & $\begin{array}{l}8 \\
8\end{array}$ & $\begin{array}{l}222 \\
\ldots 2\end{array}$ & $\begin{array}{l}F d d d(a),(b) 00 \frac{1}{2} \\
F d d 2(a)\end{array}$ \\
\hline $\mathrm{T}$ & 16 & $\overline{1}$ & $F d d d(c),(d) \frac{1}{2} \frac{1}{2} \frac{1}{2}$ \\
\hline
\end{tabular}

Orthorhombic, univariant

\begin{tabular}{|c|c|c|c|c|c|}
\hline $\mathrm{P} 2 \mathrm{x}$ & & & 2 & $2 m m$ & $\operatorname{Prmman}(i),(j) 00 \frac{1}{2},(k) 0 \frac{1}{2} 0,(2) 0 \frac{1}{2} \frac{1}{2}$ \\
\hline & & & & $2 .$. & $P 222(i),(j) 00 \frac{1}{2},(k) 0 \frac{1}{2} O,(z) 0 \frac{1}{2} \frac{1}{2}$ \\
\hline & $P 2 x[z]$ & & 2 & $\cdot m$ & $\operatorname{Pmm} 2(e),(f) O \frac{1}{2} O$ \\
\hline & P $2 y$ & $001 / 100 / 010$ & 2 & $m 2 m$ & $\operatorname{Pnmm}(m),(n) 00 \frac{1}{2},(0) \frac{1}{2} 00,(p) \frac{1}{2} 0 \frac{1}{2}$ \\
\hline & & & & .2. & $P 222(m),(n) 00 \frac{1}{2},(0) \frac{1}{2} 00,(p) \frac{1}{2} 0 \frac{1}{2}$ \\
\hline & $P 2 y[z]$ & $001 / 100 / 010$ & 2 & $m$. . & Prrm2 $(g),(h) \frac{1}{2} 00$ \\
\hline & $\mathrm{P} 2 \mathrm{z}$ & $010 / 001 / 100$ & 2 & $m m 2$ & $\operatorname{Prmmm}(q),(r) O \frac{1}{2} O,(s) \frac{1}{2} O O,(t) \frac{1}{2} \frac{1}{2} O$ \\
\hline & & & & $\ldots 2$ & $P 222(q),(r) \frac{1}{2} 00,(s) 0 \frac{1}{2} O,(t) \frac{1}{2} \frac{1}{2} O$ \\
\hline & $(P 2 x)_{C}$ & $100 / 010 / 002$ & 4 & 2. . & $\operatorname{Pccm}(i) 00 \frac{1}{4},(j) 0 \frac{1}{2} \frac{1}{4}$ \\
\hline & $(\mathrm{P} 2 \mathrm{y})_{\mathrm{a}}$ & $002 / 100 / 010$ & 4 & .2 . & $\operatorname{Pmma}(g),(h) 00 \frac{1}{2}$ \\
\hline & $(\mathrm{P} 2 \mathrm{y})_{\mathrm{c}}$ & $001 / 100 / 020$ & 4 & .2 . & $\operatorname{Pccm}(k) 00 \frac{1}{4},(Z) \frac{1}{2} O \frac{1}{4}$ \\
\hline & $(\mathrm{P} 2 \mathrm{z})_{\mathrm{C}}$ & $010 / 001 / 200$ & 4 & $\ldots 2$ & $\operatorname{Pccm}(m),(n) \frac{1}{2} \frac{1}{2} O,(0) 0 \frac{1}{2} O,(p) \frac{1}{2} 00$ \\
\hline & $(P 2 x)_{a b}$ & $200 / 020 / 001$ & 8 & 2. & $\operatorname{Cmma}(h),(i) 00 \frac{1}{2}$ \\
\hline & $(P 2 y) a b$ & $002 / 200 / 010$ & 8 & .2 . & $C m m a(j) \frac{1}{4} O O,(k) \frac{1}{4} O \frac{1}{2}$ \\
\hline & $(\mathrm{P} 2 \mathrm{z})_{\mathrm{ab}}$ & $020 / 002 / 100$ & 8 & $\ldots 2$ & $C r m m(m) \frac{1}{4} \frac{1}{4} O$ \\
\hline & & & & & Crma (Z) $\frac{1}{4} 00$ \\
\hline
\end{tabular}




\begin{tabular}{|c|c|c|c|c|}
\hline $\begin{array}{l}(\mathrm{P} 2 \mathrm{x})_{2} \\
(\mathrm{P} 2 \mathrm{y})_{2} \\
(\mathrm{P} 2 \mathrm{z})_{2}\end{array}$ & $\begin{array}{l}200 / 020 / 002 \\
002 / 200 / 020 \\
020 / 002 / 200\end{array}$ & $\begin{array}{l}16 \\
16 \\
16\end{array}$ & $\begin{array}{l}2 . \\
.2 \\
. .2\end{array}$ & $\begin{array}{l}\text { Emrmm (Z) } \frac{1}{4} \frac{1}{4} \frac{1}{4} \\
\text { Emrrom (k) } \frac{1}{4} \frac{1}{4} \frac{1}{4} \\
\text { Emrrm }(j) \frac{1}{4} \frac{1}{4} \frac{1}{4}\end{array}$ \\
\hline 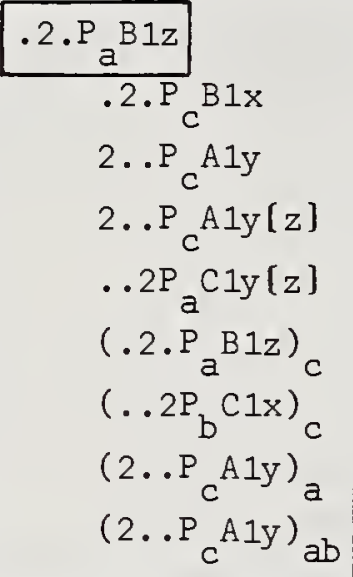 & $\begin{array}{l}00 \overline{1} / 010 / 100 \\
010 / 001 / 100 \\
010 / 001 / 100 \\
100 / 00 \overline{1} / 010 \\
100 / 010 / 002 \\
001 / 100 / 020 \\
020 / 001 / 100 \\
020 / 002 / 100\end{array}$ & $\begin{array}{l}2 \\
2 \\
2\end{array}$ & $\begin{array}{l}m m 2 \\
2 . . \\
.2 . \\
m . . \\
m_{0} . \\
. .2 \\
2 . . \\
.2 . \\
.2 .\end{array}$ & 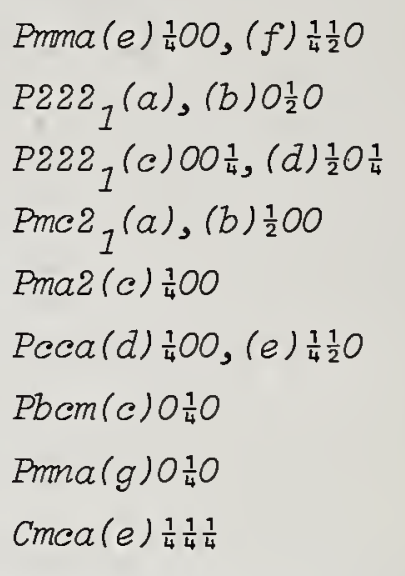 \\
\hline $\begin{array}{l}.2{ }_{1}{ }^{B I 1 y} \\
\left(2{ }_{1} \cdot C I 1 z\right)_{C}\end{array}$ & $\begin{array}{l}010 / 001 / 100 \\
100 / 010 / 002\end{array}$ & 4 & $\begin{array}{l}m m^{2} \\
\ldots 2 \\
m . . \\
\ldots 2\end{array}$ & $\begin{array}{l}\operatorname{Prmn}(a),(b) 0 \frac{1}{2} O \\
P 2_{1}{ }^{2}{ }_{1}^{2(a),(b)} 0 \frac{1}{2} O \\
\operatorname{Prn}_{2}{ }_{1}(a) \\
\operatorname{Pccn}(c) \frac{1}{4} \frac{1}{4} O,(d) \frac{1}{4} \frac{3}{4} O\end{array}$ \\
\hline $\begin{array}{l}\mathrm{C} 2 \mathrm{y}[\mathrm{z}] \\
\mathrm{B} 2 \mathrm{x} \\
\mathrm{A} 2 \mathrm{y}[\mathrm{z}] \\
(\mathrm{C} 2 \mathrm{x})_{\mathrm{C}}\end{array}$ & $\begin{array}{l}0 \overline{1} 0 / 100 / 001 \\
0 \overline{10} / 100 / 001 \\
100 / 00 \overline{1} / 010 \\
001 / 100 / 010 \\
100 / 010 / 002\end{array}$ & $\begin{array}{l}4 \\
4 \\
8\end{array}$ & $\begin{array}{l}2 m m \\
2 . . \\
. m_{\bullet} \\
m 2 m \\
.2 . \\
m . . \\
2 . . \\
m . . \\
2 . .\end{array}$ & $\begin{array}{l}\operatorname{Cmmm}(g),(h) 00 \frac{1}{2} \\
\operatorname{Pban}(g),(h) 00 \frac{1}{2} \\
\operatorname{C2} 22(e),(f) 00 \frac{1}{2} \\
\operatorname{Crmm} 2(d) \\
\operatorname{Crmm}(i),(j) 00 \frac{1}{2} \\
\operatorname{Pban}(i),(j) 00 \frac{1}{2} \\
\operatorname{C222}(g),(h) 00 \frac{1}{2} \\
\operatorname{Cmm} 2(e) \\
\operatorname{Pmna}(e),(f) 0 \frac{1}{2} 0 \\
\operatorname{Amm} 2(d),(e) \frac{1}{2} 00 \\
\operatorname{Cmcm}(e) \\
\operatorname{Cccm}(g) 00 \frac{1}{4} \\
\operatorname{Ibam}(f) 00 \frac{1}{4} \\
\operatorname{Cccm}(h) 00 \frac{1}{4} \\
\operatorname{Ibam}(g) 00 \frac{1}{4} \\
\operatorname{Imma}(f) \\
\operatorname{Imma}(g) \frac{1}{4} \frac{1}{4} \frac{1}{4}\end{array}$ \\
\hline
\end{tabular}




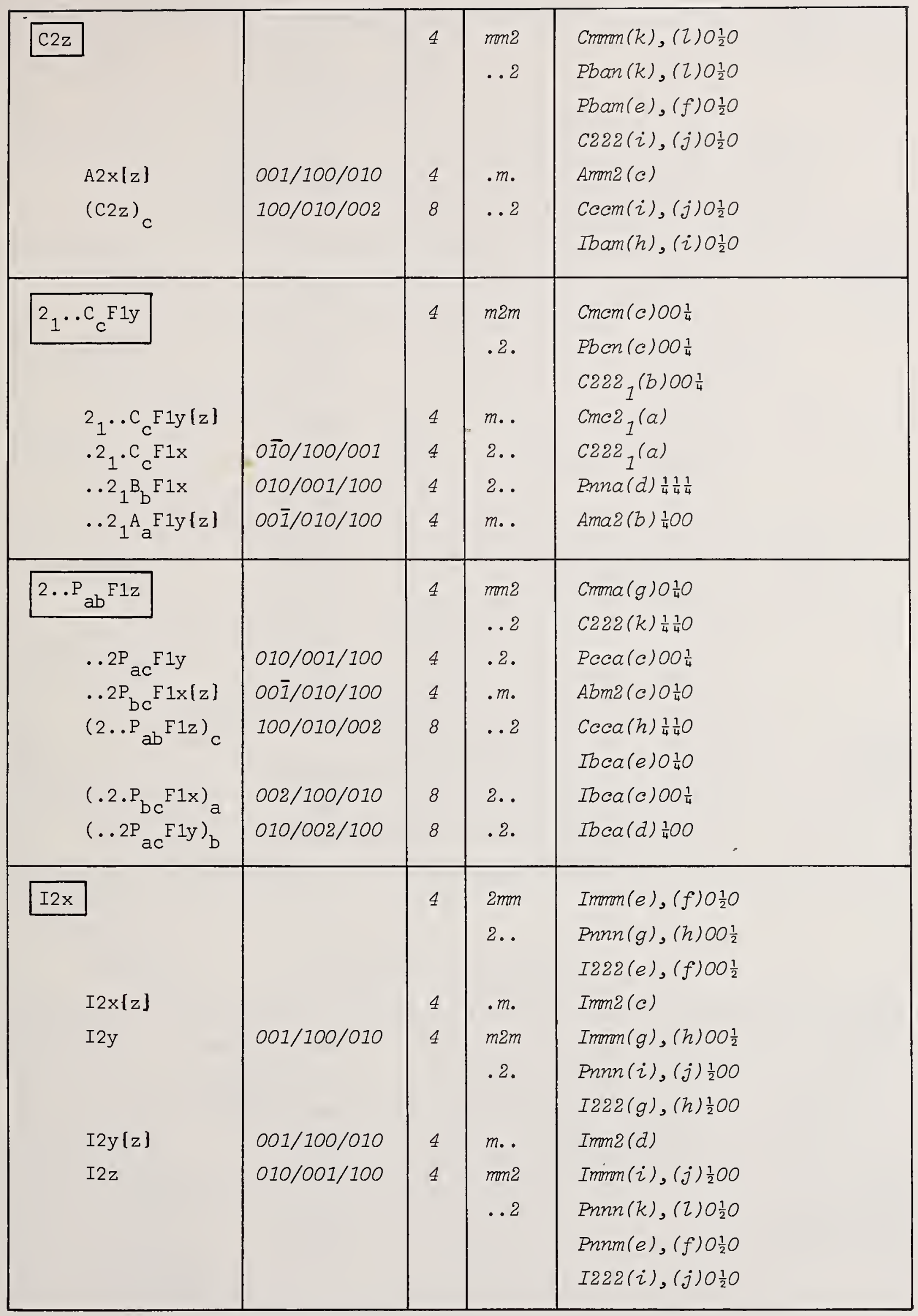




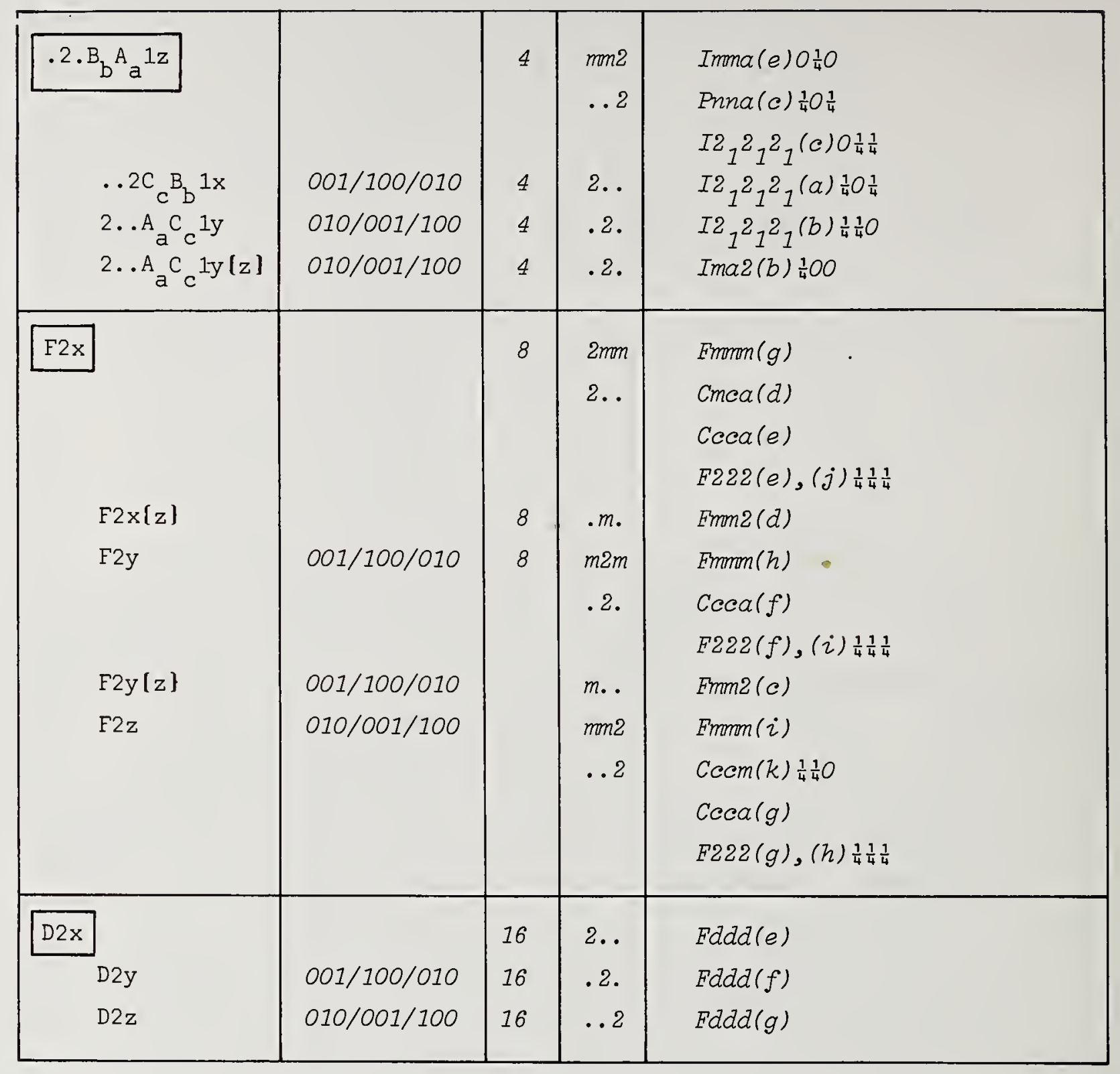

Orthorhombic, bivariant

\begin{tabular}{|c|c|c|c|c|}
\hline $\mathrm{P} 2 \mathrm{y} 2 \mathrm{z}$ & & 4 & m. . & $\operatorname{Prrman}(u),(v) \frac{1}{2} 00$ \\
\hline $\mathrm{P} 2 \mathrm{x} 2 \mathrm{z}$ & $001 / 100 / 010$ & 4 &.$m$ & $\operatorname{Prmmm}(w),(x) \bigcirc \frac{1}{2} O$ \\
\hline $\mathrm{P} 2 \mathrm{x} 2 \mathrm{y}$ & $010 / 001 / 100$ & 4 & $\ldots m$ & $\operatorname{Pntrmm}(y),(z) 00 \frac{1}{2}$ \\
\hline $\mathrm{P} 2 \mathrm{x} 2 \mathrm{y}[\mathrm{z}]$ & $010 / 001 / 100$ & 4 & 1 & $\operatorname{Prm} 2(i)$ \\
\hline $2 \ldots \mathrm{P}_{\mathrm{c}} 2 \mathrm{xy}$ & & 4 & $\ldots m$ & $\operatorname{Pccm}(q)$ \\
\hline $2 \ldots P c^{2 x y}[z]$ & & 4 & 1 & $\operatorname{Pcc} 2(e)$ \\
\hline
\end{tabular}




\begin{tabular}{|c|c|c|c|c|}
\hline$\frac{\frac{\mathrm{m} . \mathrm{P}_{a} 2 \mathrm{xz}}{\mathrm{m} . . \mathrm{P}_{\mathrm{a}} 2 x y\{z\}}}{}$ & $100 / 00 \overline{1} / 010$ & 4 & $\begin{array}{l}\cdot m \cdot \\
1\end{array}$ & $\begin{array}{l}\operatorname{Pmma}(i),(j) O \frac{1}{2} O \\
\operatorname{Pma2}(d)\end{array}$ \\
\hline$\frac{.2 . P_{a} B 1 z 2 y}{2 \ldots P_{c}{ }^{A} 1 y 2 x[z]}$ & $010 / 001 / 100$ & $\begin{array}{l}4 \\
4\end{array}$ & $\begin{array}{l}m_{0} . \\
1\end{array}$ & $\begin{array}{l}\operatorname{Pmma}(k) \frac{100}{4} 00 \\
\operatorname{Pmer}_{1}(c)\end{array}$ \\
\hline$\frac{.2 . \mathrm{B} 2 \mathrm{yz}}{2 \ldots \mathrm{A} 2 \mathrm{xy}[\mathrm{z}]}$ & $010 / 001 / 100$ & $\begin{array}{l}4 \\
4\end{array}$ & $\begin{array}{l}m_{0} . \\
1\end{array}$ & $\begin{array}{l}\operatorname{Prna}(h) \\
\operatorname{Pnc2}(c)\end{array}$ \\
\hline$\frac{b . . c 2 x y}{b \ldots c 2 x y[z]}$ & & $\begin{array}{l}4 \\
4\end{array}$ & $\begin{array}{l}. . m \\
1\end{array}$ & $\begin{array}{l}\operatorname{Pbam}(g),(h) 00 \frac{1}{2} \\
\operatorname{Pba2}(c)\end{array}$ \\
\hline$\frac{2 . \overline{1} P_{b c} A_{b} c_{c} F 1 x y}{.2 \overline{1} P_{a c}{ }^{B} a^{C} c^{F} 1 x y\{z\}}$ & $0 \overline{10 / 100 / 001}$ & $\begin{array}{l}4 \\
4\end{array}$ & $\begin{array}{l}. . m \\
1\end{array}$ & $\begin{array}{l}\operatorname{Pbcm}(d) 00 \frac{1}{4} \\
\operatorname{Pcar}_{1}(a)\end{array}$ \\
\hline$\frac{n . . I 2 x y}{n . . I 2 x y(z)}$ & & $\begin{array}{l}4 \\
4\end{array}$ & $\begin{array}{l}. . m \\
1\end{array}$ & $\begin{array}{l}\text { Pnnm (g) } \\
\text { Pnn2 (c) }\end{array}$ \\
\hline 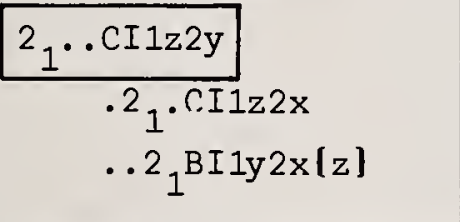 & $\begin{array}{l}0 \overline{10} / 100 / 001 \\
010 / 001 / 100\end{array}$ & $\begin{array}{l}4 \\
4 \\
4\end{array}$ & $\begin{array}{l}m . . \\
. m . \\
1\end{array}$ & $\begin{array}{l}\operatorname{Pmmn}(e) \\
\operatorname{Pmmn}(f) \\
\operatorname{Prnn} 2_{1}(b)\end{array}$ \\
\hline$\frac{\overline{1} \cdot{ }_{1} \mathrm{~B}_{b} \mathrm{~A}_{a} \mathrm{FI}_{a} 1 \mathrm{xz}}{\overline{1}{ }_{1} \cdot{ }{ }_{c}{ }_{\mathrm{A}} \mathrm{FI}_{\mathrm{a}} 1 \mathrm{xy}[\mathrm{z}]}$ & $100 / 00 \overline{1} / 010$ & $\begin{array}{l}4 \\
4\end{array}$ & $\begin{array}{l}. m \cdot \\
1\end{array}$ & $\begin{array}{l}\operatorname{Pnma}(c) 0 \frac{1}{4} O \\
\operatorname{Pna2}_{1}(a)\end{array}$ \\
\hline $\begin{array}{l}\frac{C 2 y 2 z}{C 2 x 2 z} \\
A 2 x 2 y\{z\}\end{array}$ & $\begin{array}{l}0 \overline{1} 0 / 100 / 001 \\
00 \overline{1} / 010 / 100\end{array}$ & $\begin{array}{l}8 \\
8\end{array}$ & $\begin{array}{l}m_{.} . \\
. m . \\
1\end{array}$ & $\begin{array}{l}\operatorname{Crmmm}(n) \\
\operatorname{Crmm}(o) \\
\operatorname{Amm2}(f)\end{array}$ \\
\hline$\underbrace{C 2 x 2 y}_{C 2 x 2 y[z]}$ & & $\begin{array}{l}8 \\
8\end{array}$ & $\begin{array}{l}. . m \\
1\end{array}$ & $\begin{array}{l}\operatorname{Cmmm}(p),(q) 00 \frac{1}{2} \\
\operatorname{Crm} 2(f)\end{array}$ \\
\hline$\frac{n_{c}^{. n \cdot c_{c} 2 y z}}{. \cdot n A_{a} 2 x y[z]}$ & $00 \overline{1} / 010 / 100$ & $\begin{array}{l}8 \\
8\end{array}$ & $\begin{array}{l}m_{0} . \\
1\end{array}$ & $\begin{array}{l}\operatorname{Cmem}(f) \\
\operatorname{Ama2}(c)\end{array}$ \\
\hline$\frac{2_{1} \ldots C_{C}{ }^{F 1 y 2 x}}{2_{1}{ } \cdot C_{C}{ }_{C} 1 y 2 x[z]}$ & & $\begin{array}{l}8 \\
8\end{array}$ & $\begin{array}{l}. . m \\
1\end{array}$ & $\begin{array}{l}\operatorname{Cmem}(g) 00 \frac{1}{4} \\
\operatorname{Cmer}_{1}(b)\end{array}$ \\
\hline
\end{tabular}




\begin{tabular}{|c|c|c|c|c|}
\hline$\frac{.2 . F 2 y z]}{.2 . F 2 x y[z]}$ & $00 \overline{1} / 010 / 100$ & $\begin{array}{l}8 \\
8\end{array}$ & $\begin{array}{l}m . \\
1\end{array}$ & $\begin{array}{l}\operatorname{Cmca}(f) \\
A b a 2(b)\end{array}$ \\
\hline$\frac{n \ldots C_{C} F 2 x y}{n \ldots C_{C} F 2 x y[z]}$ & & $\begin{array}{l}8 \\
8\end{array}$ & $\begin{array}{l}\ldots m \\
1\end{array}$ & $\begin{array}{l}\operatorname{Cccm}(z) \\
\operatorname{Ccc} 2(d)\end{array}$ \\
\hline $\begin{array}{l}\frac{{ }^{m \cdot P} a b^{2 y z}}{m \cdot \cdot P} P_{a b} 2 x z \\
\cdot m \cdot P_{b c} 2 x y[z]\end{array}$ & $\begin{array}{l}0 \overline{10} / 100 / 001 \\
00 \overline{1} / 010 / 100\end{array}$ & $\begin{array}{l}8 \\
8\end{array}$ & $\begin{array}{l}m . . \\
. m . \\
1\end{array}$ & $\begin{array}{l}\text { Crma }(m) \\
\text { Crma (n)O } \frac{1}{4} 0 \\
\text { Abm2 (d) }\end{array}$ \\
\hline $\begin{array}{l}I 2 y 2 z \\
I 2 x 2 z \\
I 2 x 2 y \\
I 2 x 2 y[z]\end{array}$ & $\begin{array}{l}001 / 100 / 010 \\
010 / 001 / 100 \\
010 / 001 / 100\end{array}$ & $\begin{array}{l}8 \\
8 \\
8 \\
8\end{array}$ & $\begin{array}{l}m . . \\
. m . \\
\ldots m \\
1\end{array}$ & $\begin{array}{l}\operatorname{Immm}(Z) \\
\operatorname{Immm}(m) \\
\operatorname{Irmmm}(n) \\
\operatorname{Imon2}(e)\end{array}$ \\
\hline$\frac{b \cdot c_{c}^{2 x y}}{b \ldots c_{c} 2 x y[z]}$ & & $\begin{array}{l}8 \\
8\end{array}$ & $\begin{array}{l}\ldots m \\
1\end{array}$ & $\begin{array}{l}\operatorname{Ibam}(j) \\
\operatorname{Iba} 2(c)\end{array}$ \\
\hline $\begin{array}{l}\frac{.2 \cdot b_{b} 2 y z}{2 \ldots A} a^{2 x z} \\
2 \ldots a_{a} 2 x y[z]\end{array}$ & $\begin{array}{l}0 \overline{1} 0 / 100 / 001 \\
010 / 001 / 100\end{array}$ & $\begin{array}{l}8 \\
8 \\
8\end{array}$ & $\begin{array}{l}m . . \\
. m . \\
1\end{array}$ & $\begin{array}{l}\operatorname{Imma}(h) \\
\operatorname{Imma}(i) \frac{1}{4} \frac{1}{4} \frac{1}{4} \\
\operatorname{Ima2}(c)\end{array}$ \\
\hline $\begin{array}{l}F 2 y 2 z \\
F 2 x 2 z \\
F 2 x 2 y \\
F 2 x 2 y[z]\end{array}$ & $\begin{array}{l}001 / 100 / 010 \\
010 / 001 / 100 \\
010 / 001 / 100\end{array}$ & $\begin{array}{l}16 \\
16 \\
16 \\
16\end{array}$ & $\begin{array}{l}m . . \\
. m . \\
\ldots m \\
1\end{array}$ & $\begin{array}{l}\text { Frmmm }(m) \\
\text { Entrmm }(n) \\
\text { Entrm }(0) \\
\text { Entm2 }(e)\end{array}$ \\
\hline d...D2xy $\{z\}$ & & 16 & 1 & $F d d 2(b)$ \\
\hline
\end{tabular}


The 4-pointers:

\begin{tabular}{|c|c|}
\hline $\mathrm{P} 2 \mathrm{x} 2 \mathrm{yz}$ & P222(u) \\
\hline 2.P $\mathrm{C}^{\mathrm{B} 1 \mathrm{x} 2 \mathrm{yz}}$ & $P 222_{1}(e)$ \\
\hline${ }^{2} \ldots \operatorname{CI} 1 \mathrm{z} 2 \mathrm{xy}$ & $P 2_{1}^{2} 1^{2(c)}$ \\
\hline${ }^{2}{ }^{2}{ }_{1} \cdot F A_{a} B_{b} C_{c} I_{a} I_{b} I_{c} 1 x y z$ & $\mathrm{P}_{1}{ }^{2} 1^{2}{ }_{1}^{(a)}$ \\
\hline
\end{tabular}

The 8-pointers:

\begin{tabular}{|c|c|}
\hline $\mathrm{P} 2 \mathrm{x} 2 \mathrm{y} 2 \mathrm{z}$ & $\operatorname{Pmmm}(\alpha)$ \\
\hline n... $2 x 2 y z$ & $\operatorname{Pnnn}(m)$ \\
\hline$\cdots \mathrm{mP} c c^{2 \mathrm{x} 2 \mathrm{yz}}$ & $\operatorname{Pccm}(r) 00 \frac{1}{4}$ \\
\hline b. . C $2 x 2 y z$ & $\operatorname{Pban}(m)$ \\
\hline $\mathrm{m} . . \mathrm{P} \mathrm{a}^{2 \mathrm{xz} 2 \mathrm{y}}$ & Prma (2) \\
\hline $2.2 A_{a} 2 x y z$ & Pnna(e) \\
\hline $.2 . \mathrm{B} 2 \mathrm{yz} 2 \mathrm{x}$ & $\operatorname{Prna}(i)$ \\
\hline $.22 \mathrm{P} a c^{2 x y z}$ & $\operatorname{Pcca}(f)$ \\
\hline b..C2xy $2 z$ & $\operatorname{Pbam}(i)$ \\
\hline c. $2 \mathrm{~F} 2 \mathrm{xyz}$ & $\operatorname{Pccn}(e)$ \\
\hline 2. $\mathrm{mP}_{\mathrm{bc}} 2 \mathrm{xyz}$ & $P b c m(e)$ \\
\hline n... $2 x y 2 z$ & $\operatorname{Pnnm}(h)$ \\
\hline${ }^{2}{ }_{1}$.CI $1 z 2 x 2 y$ & Prmm (g) \\
\hline
\end{tabular}

\begin{tabular}{|c|c|}
\hline $\mathrm{b} 2 \cdot \mathrm{c}^{2 \mathrm{xyz}}$ & $\operatorname{Pbcn}(d)$ \\
\hline bc.F $2 x y z$ & $P b c a(c)$ \\
\hline - $\mathrm{maB}_{\mathrm{b}} 2 x y z$ & Pnma(d) \\
\hline $\mathrm{C} 2 \mathrm{x} 2 \mathrm{yz}$ & $C 222(2)$ \\
\hline$\cdot{ }^{2}{ }_{1} \cdot C_{c} F 1 x 2 y z$ & $C 222_{1}(c)$ \\
\hline $\mathrm{I} 2 \mathrm{x} 2 \mathrm{yz}$ & I222(k) \\
\hline$\ldots 2 C_{c} B_{b} 1 x 2 y z$ & $I 2_{1}^{2} 1^{2}{ }^{(d)} \frac{1}{4} O \frac{1}{4}$ \\
\hline
\end{tabular}

The 16-pointers:

\begin{tabular}{|c|c|}
\hline$C 2 \times 2 y 2 z$ & $C m m n(r)$ \\
\hline .n. $C_{c} 2 y z 2 x$ & $\operatorname{Cmem}(h)$ \\
\hline . 2. F $2 y z 2 x$ & Cmea $(g)$ \\
\hline n... $c_{C} 2 \mathrm{x} 2 \mathrm{yz}$ & $\operatorname{Cccm}(\mathrm{m}) 00 \frac{7}{4}$ \\
\hline m... ${ }_{a b} 2 x 2 y z$ & Crma (0) $\frac{1}{4} 00$ \\
\hline c..F 2 x $2 y z$ & $\operatorname{Ccc} \alpha(i)$ \\
\hline$I 2 x 2 y 2 z$ & $\operatorname{Inmmm}(0)$ \\
\hline b...c $2 x 2 y z$ & $\operatorname{Ibam}(k) 00 \frac{1}{4}$ \\
\hline $22 \cdot P 22 x y z$ & $\operatorname{Ibca}(f)$ \\
\hline $.2 . B_{b} 2 y z 2 x$ & $\operatorname{Imma}(j)$ \\
\hline
\end{tabular}




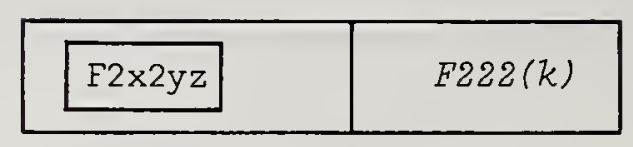

The 32-pointers:

\begin{tabular}{|l|l|}
\hline F2x2y2z & Frrm $(p)$ \\
\hline d..D2x2yz & Fddd $(h)$ \\
\hline
\end{tabular}


Table 30

\begin{tabular}{|l|l|l|l|}
\hline $\begin{array}{l}\text { Lattice complex } \\
\text { in all its } \\
\text { representations }\end{array}$ & $\begin{array}{l}\text { Multi- } \\
\text { plicity }\end{array}$ & $\begin{array}{l}\text { Site } \\
\text { symmetry } \\
\text { (oriented) }\end{array}$ & $\begin{array}{l}\text { Occurrence: } \\
\text { space group, Wyckoff letter, } \\
\text { shift from standard representation }\end{array}$ \\
\hline
\end{tabular}

Monoclinic, invariant

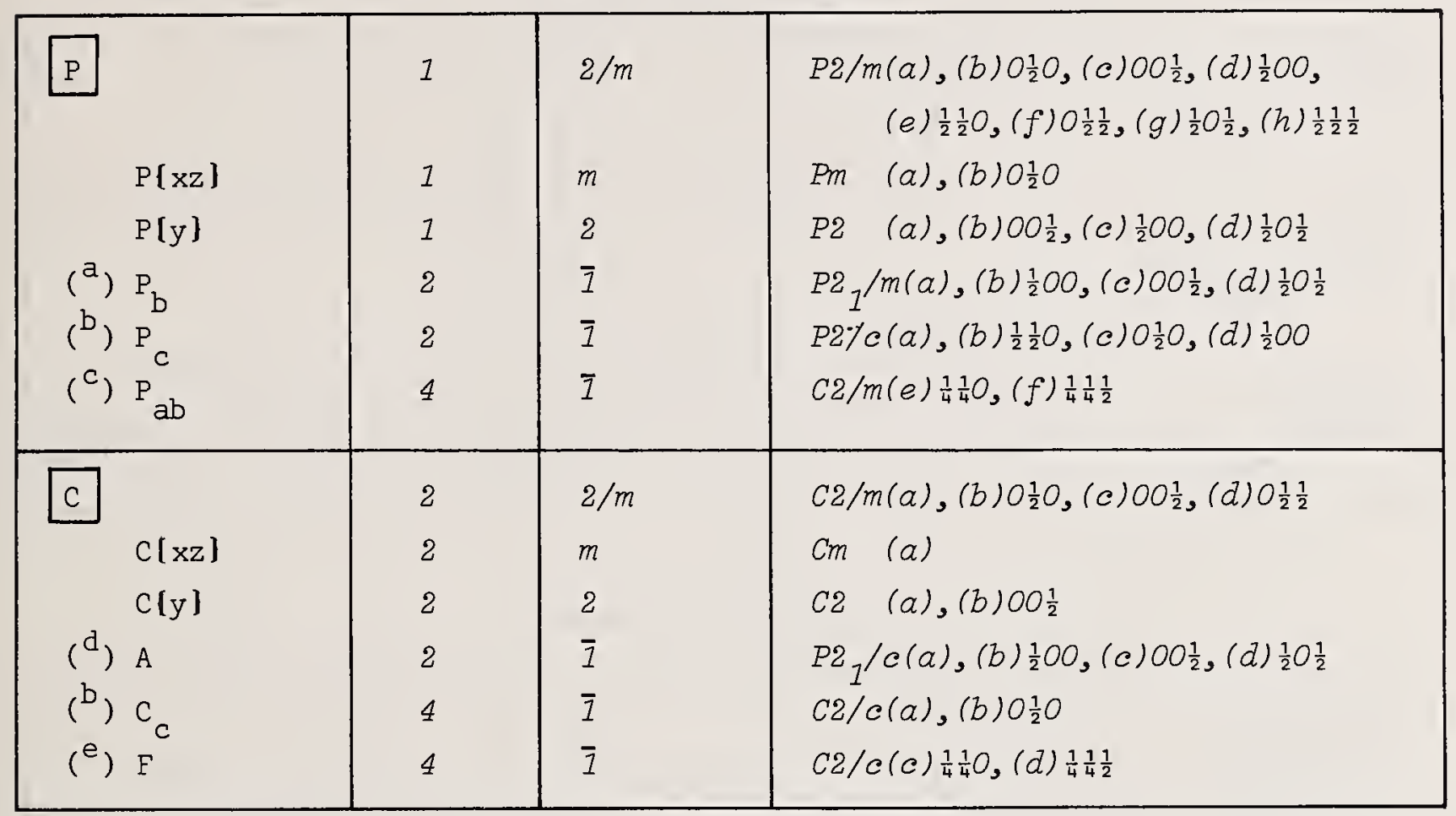

Monoclinic, univariant

\begin{tabular}{|c|c|c|c|}
\hline$P 2 y \underbrace{}_{P 2 y}\{x z\}$ & $\begin{array}{l}2 \\
2\end{array}$ & $\begin{array}{l}2 \\
1\end{array}$ & $\begin{array}{l}P 2 / m(i),(j) \frac{1}{2} 00,(k) O O \frac{1}{2},(l) \frac{1}{2} O \frac{1}{2} \\
P m \quad \text { (c) }\end{array}$ \\
\hline$\frac{{ }^{C P}{ }_{c}^{A} 1 y}{C P}{ }_{c}^{A} 1 y\{x z\}$ & $\begin{array}{l}2 \\
2\end{array}$ & $\begin{array}{l}2 \\
1\end{array}$ & $\begin{array}{l}P 2 / C(e) 00 \frac{1}{4},(f) \frac{1}{2} O \frac{1}{4} \\
P C \quad(a)\end{array}$ \\
\hline $\mathrm{C} 2 \mathrm{y}\{\mathrm{xz}\}$ & $\begin{array}{l}4 \\
4\end{array}$ & 2 & 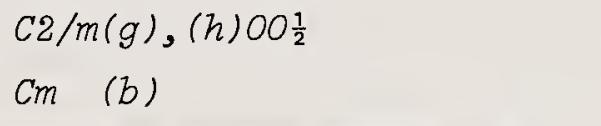 \\
\hline
\end{tabular}

( $\left.{ }^{a}\right) 100 / 020 / 001 .\left({ }^{b}\right) 100 / 010 / 002 \cdot\left({ }^{c}\right) 200 / 020 / 001 .\left({ }^{d}\right) 001 / 010 / 100 .\left({ }^{e}\right) 100 / 010 / 102$. From standard representation to cell. 


\begin{tabular}{|l|l|l|l|}
\hline$\overline{1} C_{C} F 1 y$ \\
\hline$\overline{1} C_{C} F 1 y[x z]$ & 4 & 1 & $C 2 / c(e) 00 \frac{1}{4}$ \\
\hline
\end{tabular}

Monoclinic, bivariant

\begin{tabular}{|c|c|c|c|}
\hline$\frac{P 2 x z}{P 2 x z[y]}$ & 2 & $m$ & $\begin{array}{l}P 2 / m(m),(n) 0 \frac{1}{2} O \\
P 2 \text { (e) }\end{array}$ \\
\hline 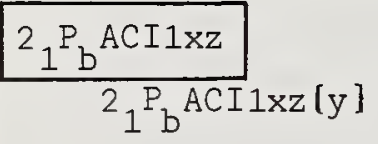 & 2 & $m$ & $\begin{array}{l}P 2_{1} / m(e) 0 \frac{1}{4} 0 \\
P 2_{1}(a)\end{array}$ \\
\hline$\frac{\mathrm{C} 2 \mathrm{xz}}{\mathrm{C} 2 \mathrm{xz}[\mathrm{y}]}$ & 4 & $m$ & $\begin{array}{l}c 2 / m(i) \\
c 2 \quad(c)\end{array}$ \\
\hline
\end{tabular}

Monoclinic, trivariant

\begin{tabular}{|l|l|l|l|}
\hline $\mathrm{P} 2 \mathrm{xz} 2 \mathrm{y}$ & 4 & 1 & $P 2 / m(0)$ \\
\hline $\mathrm{mP}{ }_{\mathrm{b}} 2 \mathrm{xyz}$ & 4 & 1 & $P 2{ }_{1} / m(f)$ \\
\hline $2 \mathrm{P}_{\mathrm{c}} 2 \mathrm{xyz}$ & 4 & 1 & $P 2 / c(g)$ \\
\hline $\mathrm{CA} 2 \mathrm{xyz}$ & 4 & 1 & $P_{1} / c(e)$ \\
\hline $\mathrm{C} 2 \mathrm{xz} 2 \mathrm{y}$ & 8 & 1 & $C 2 / m(j)$ \\
\hline $2{ }_{1}{ }_{c} \mathrm{~F} 2 \mathrm{xyz}$ & 8 & 1 & $C 2 / c(f)$ \\
\hline
\end{tabular}


Table 31

\begin{tabular}{|c|c|c|c|}
\hline $\begin{array}{l}\text { Lattice complex } \\
\text { in all its } \\
\text { representations }\end{array}$ & $\begin{array}{l}\text { Multi- } \\
\text { plicity }\end{array}$ & $\begin{array}{l}\text { Site } \\
\text { symmetry } \\
\text { (oriented) }\end{array}$ & $\begin{array}{l}\text { Occurrence: } \\
\text { space group, Wyckoff letter, } \\
\text { shift from standard representation }\end{array}$ \\
\hline
\end{tabular}

Anorthic (triclinic), invariant

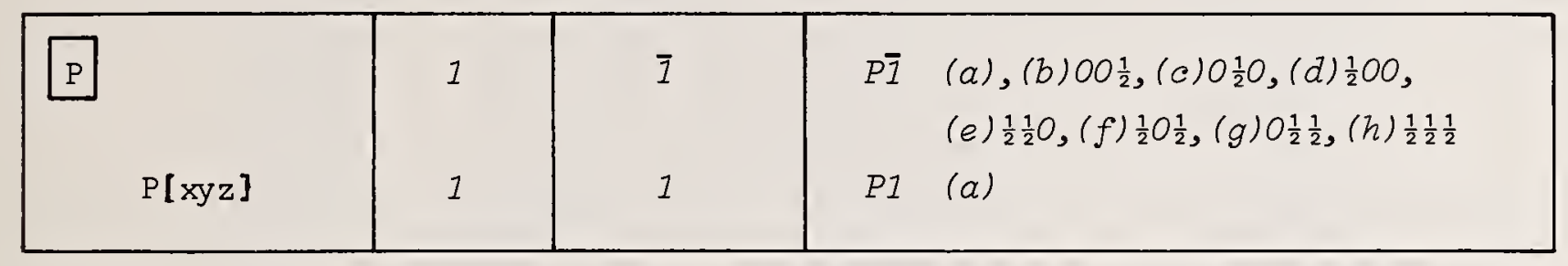

Anorthic (triclinic), trivariant

\begin{tabular}{|l|l|l|l|}
\hline P2xyz & 2 & 1 & $P \overline{1} \quad(i)$ \\
\hline
\end{tabular}



$\int_{0}$

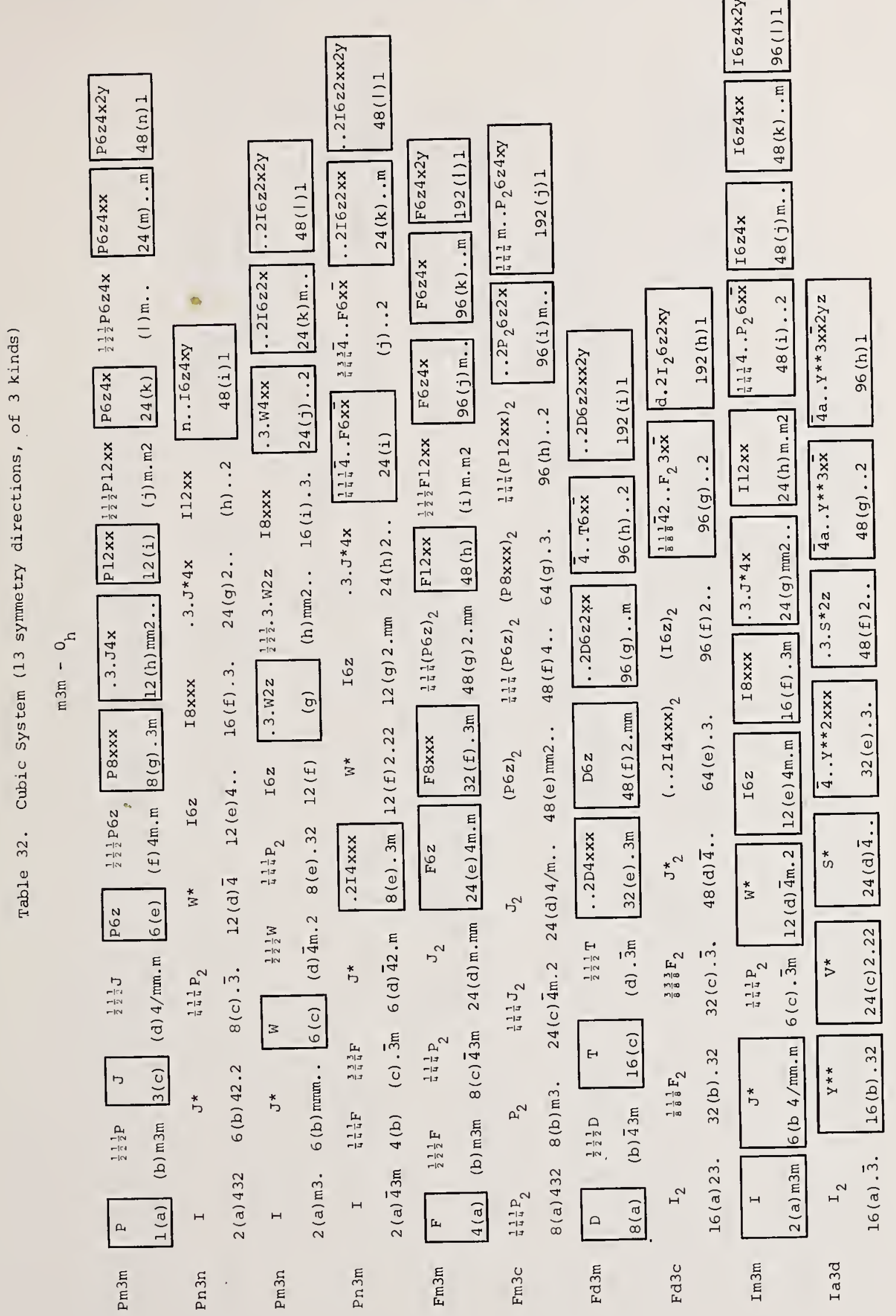




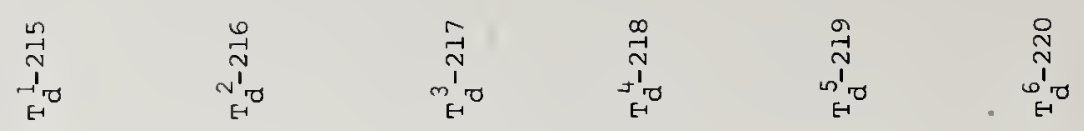

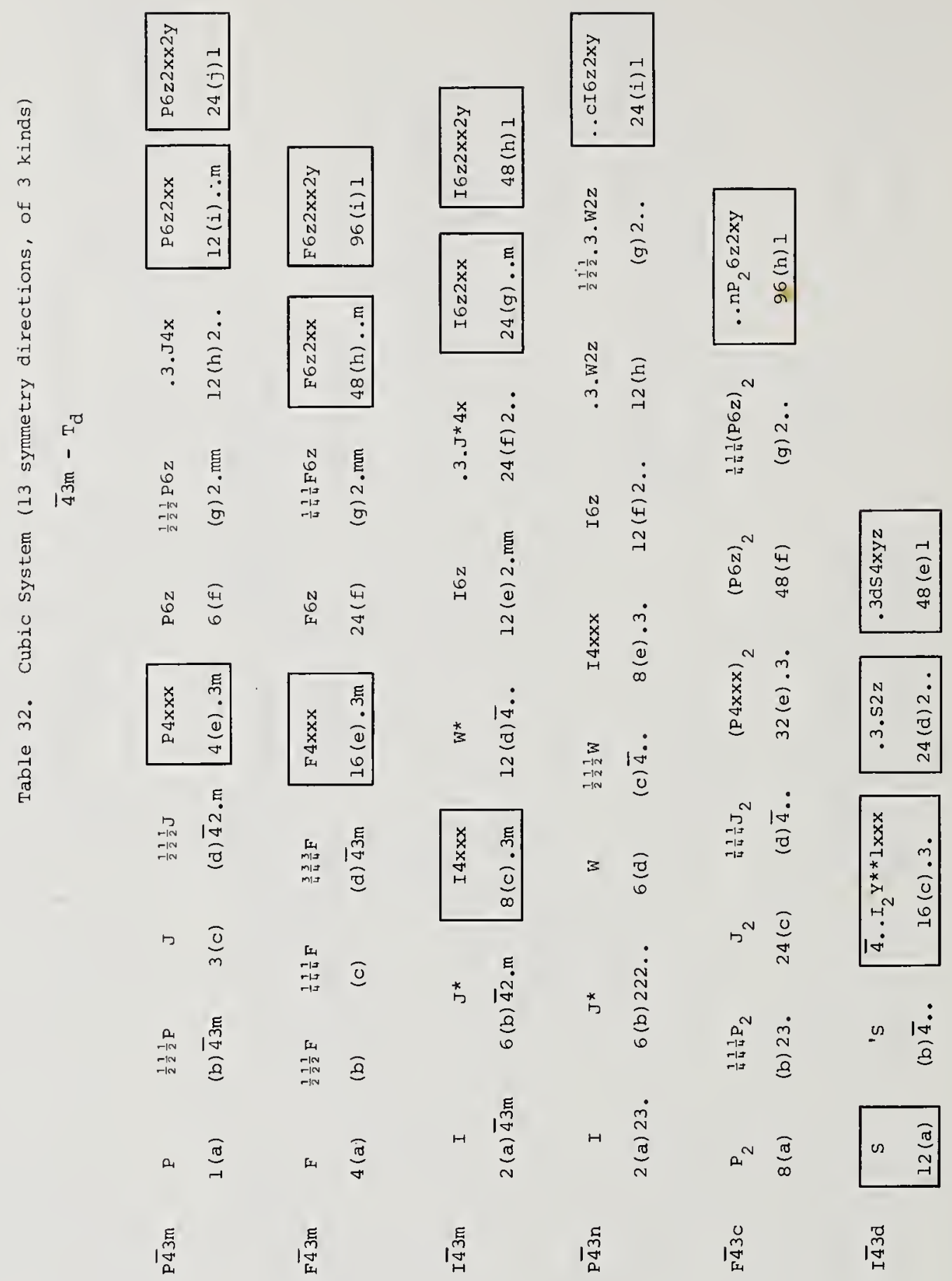




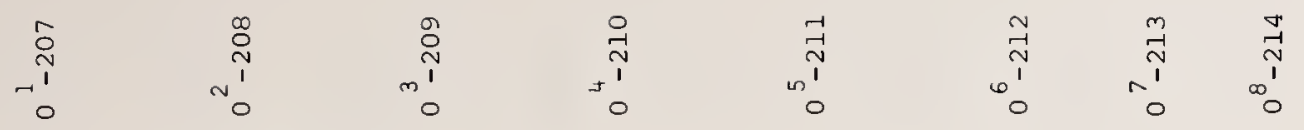

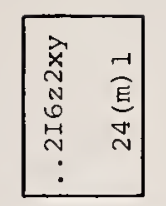

党

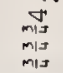

高

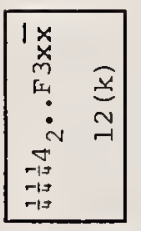

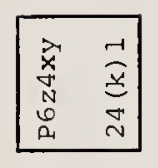

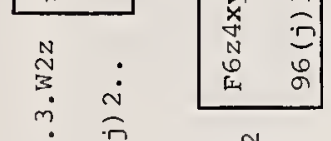

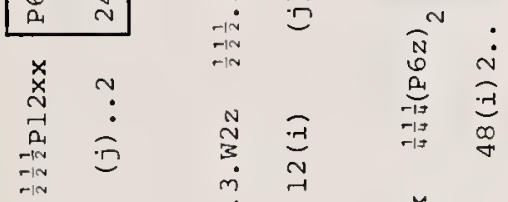

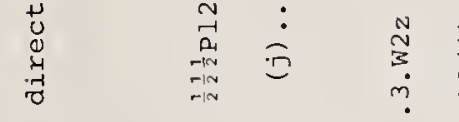

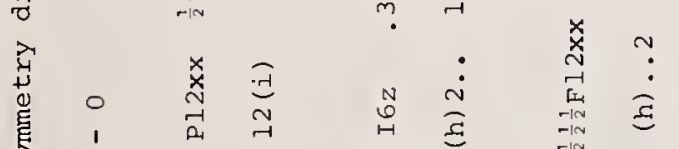

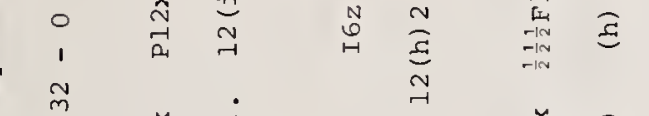

$\stackrel{m}{\rightleftharpoons} \stackrel{m}{\sigma}$

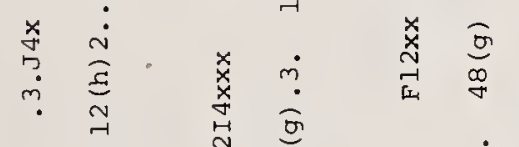

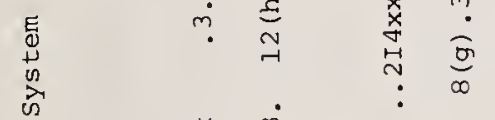

高

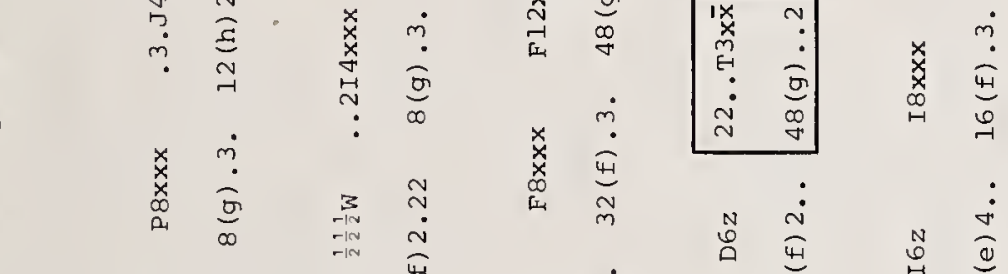

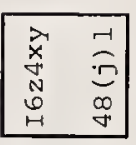

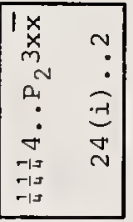

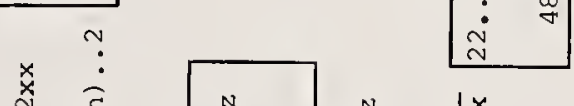

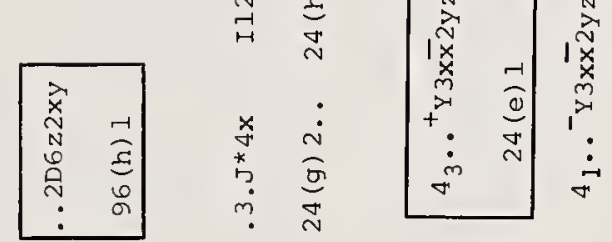

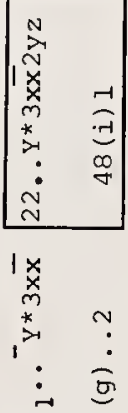

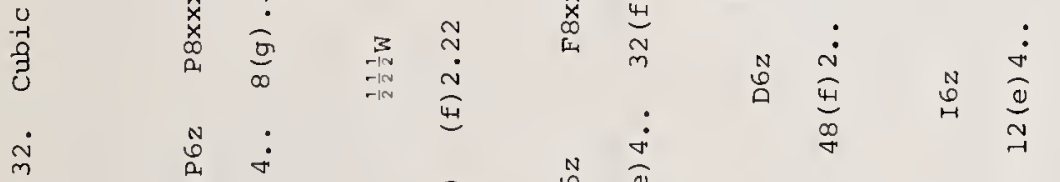

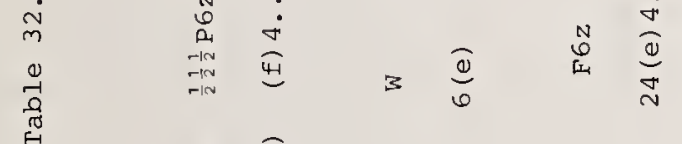

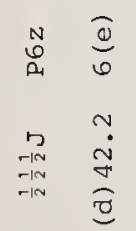

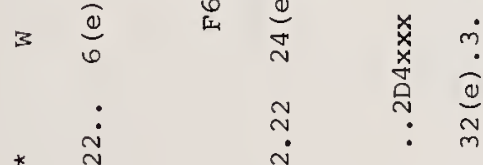

$\cdot 3$

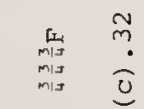

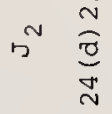

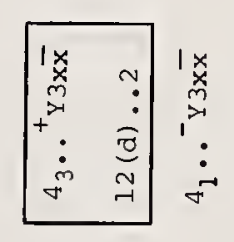

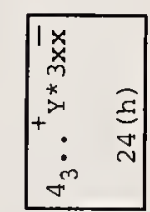

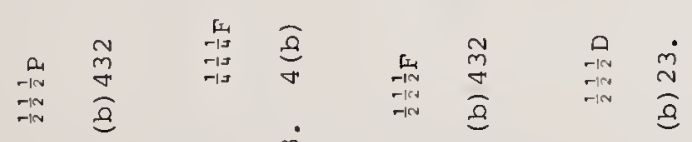

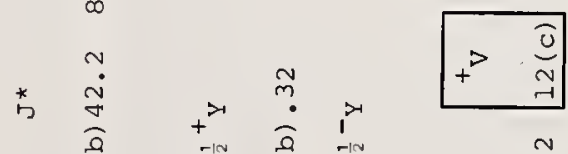

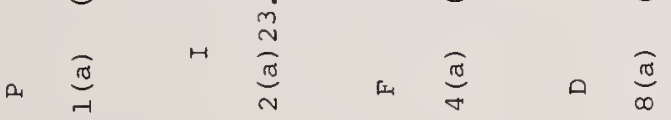

$+\underset{\substack{N \\ ָ}}{\stackrel{N}{\pi}}$

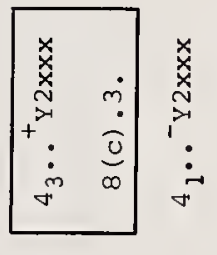

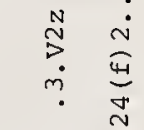

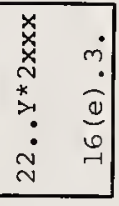

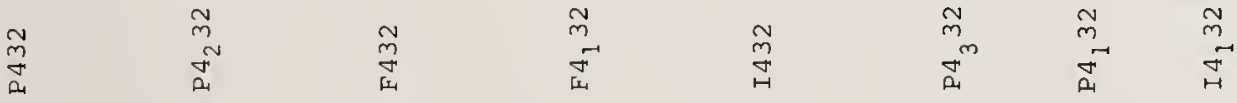




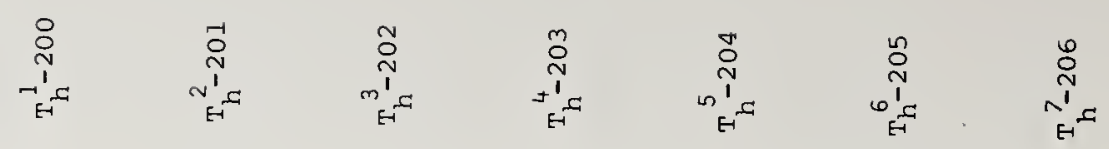

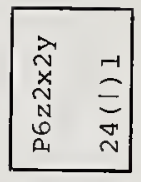

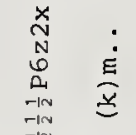
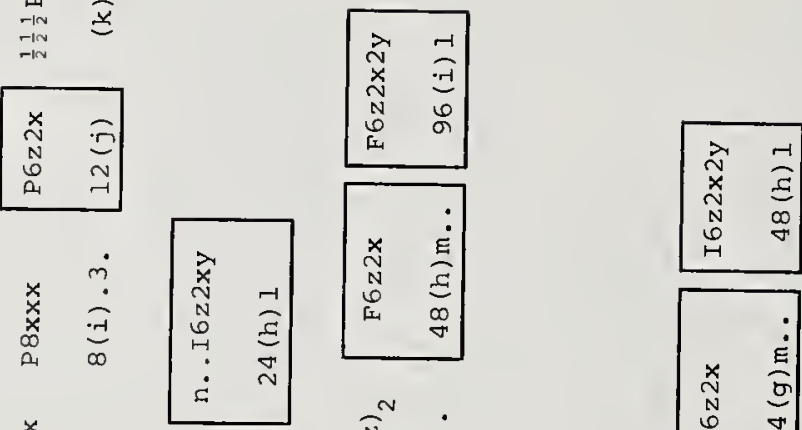

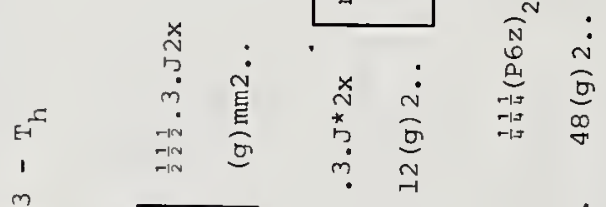

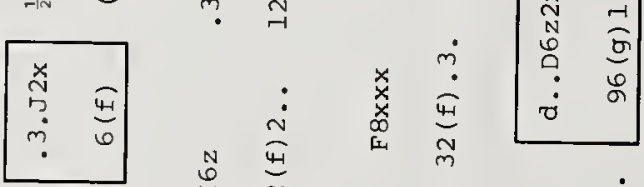

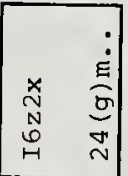

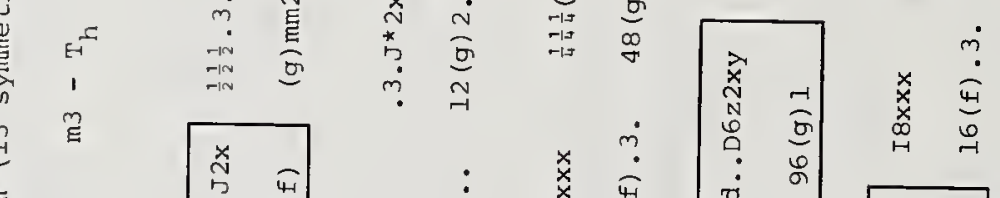

焉

लं

:

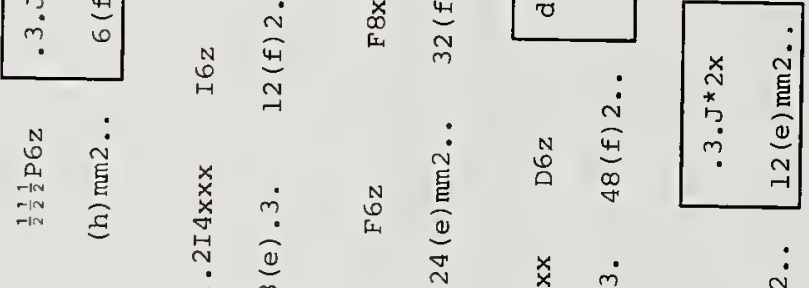

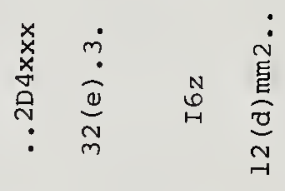

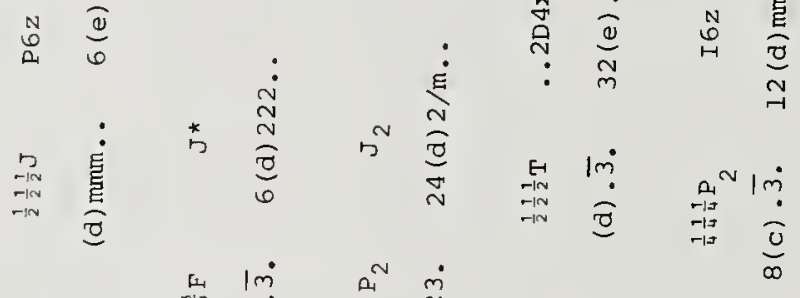

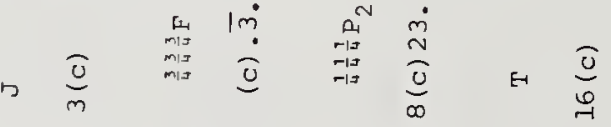

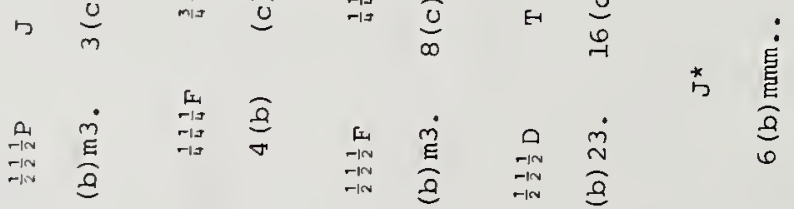

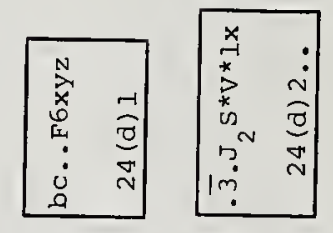

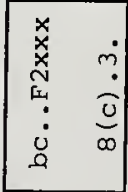

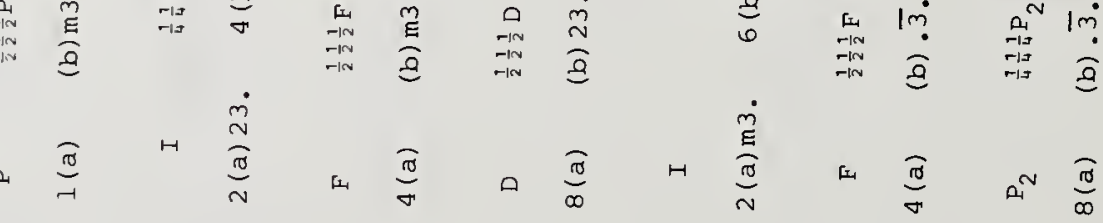

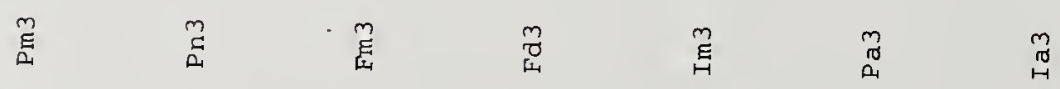


$\underset{-1}{\stackrel{2}{-1}}$

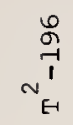

مै

$\infty$
$\stackrel{1}{-1}$
+1
-1

$\frac{9}{2}$

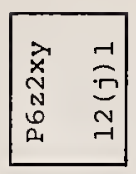

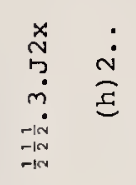

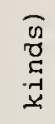

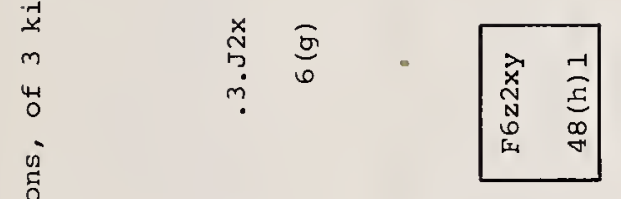

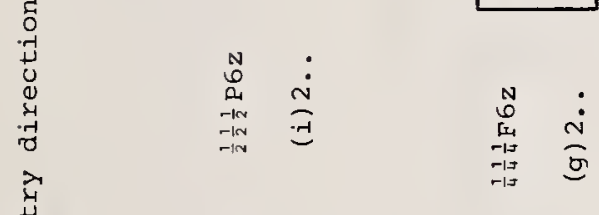

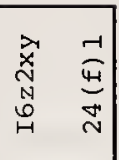

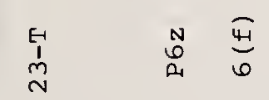

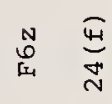

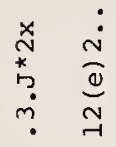

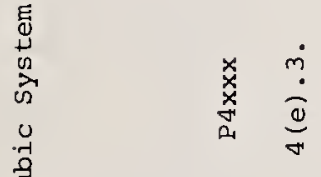

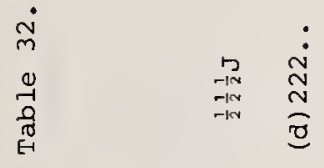

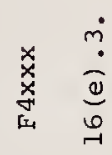

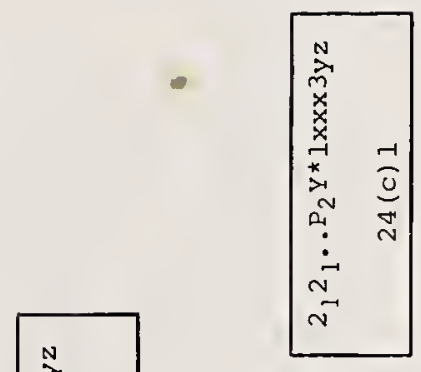

$\rightarrow \frac{\hat{m}}{3}$

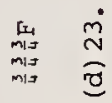

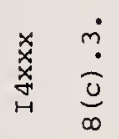

$\prod_{\substack{-10 \\-10}} 0$

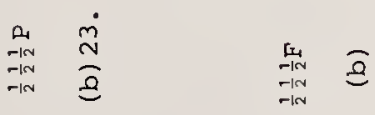

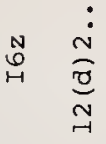

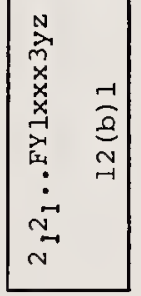

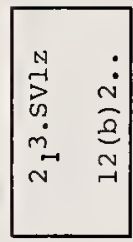

a. $\quad \underbrace{\pi}_{-1}$

4

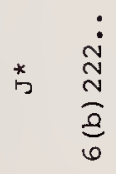

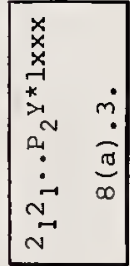

$\stackrel{m}{a}$

$\underset{\mathfrak{m}}{\mathfrak{m}}$

$\stackrel{m}{r}$

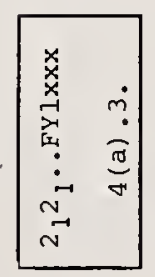

$\stackrel{m}{n}$

$\stackrel{m}{n}$ 


\section{5
$\frac{9}{1}$
$-\frac{5}{6}$}

๑ั

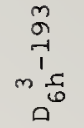

5
7
7
$+\frac{1}{6}$
0

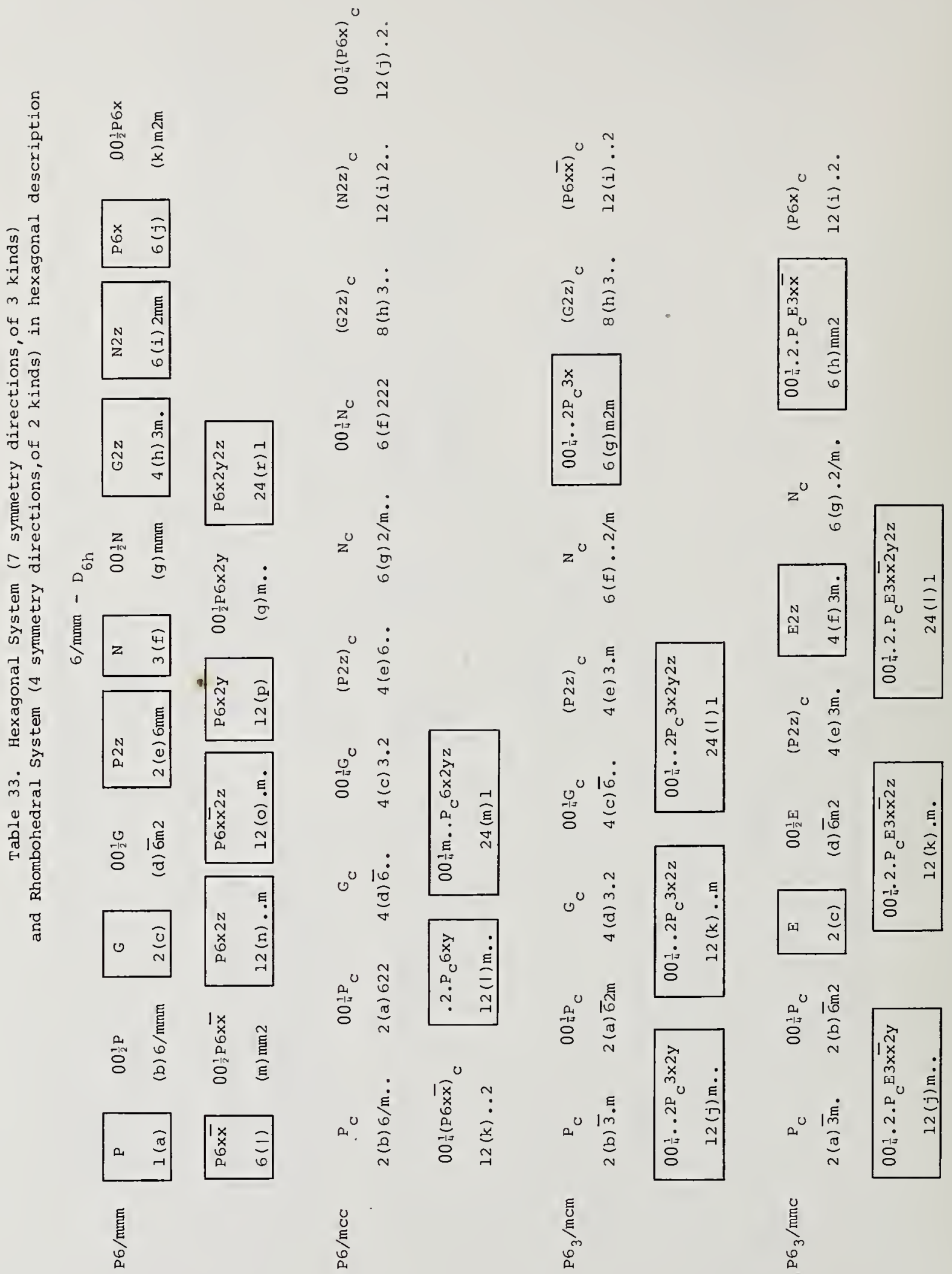


产

量

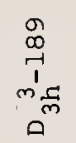

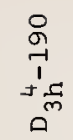
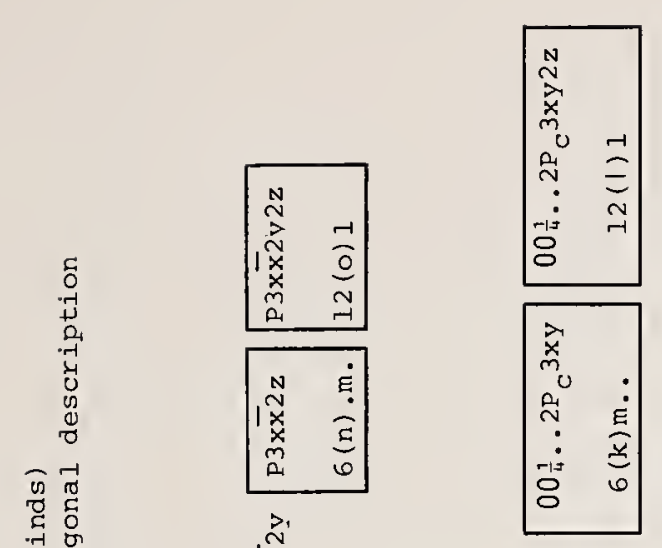

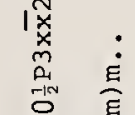

फ़

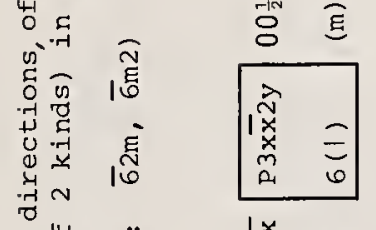

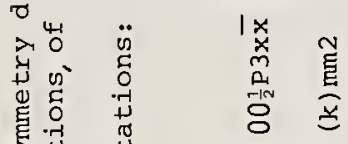

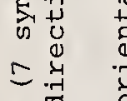

हु

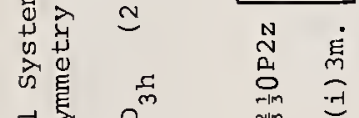

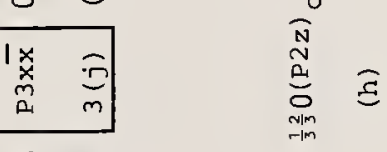

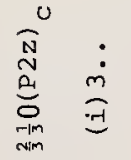
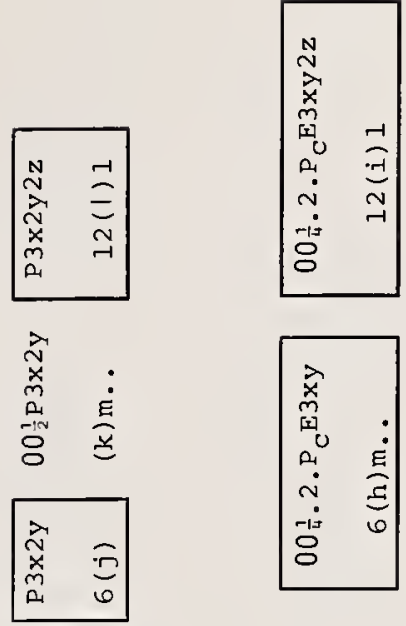

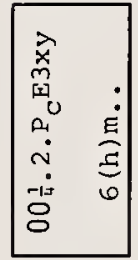

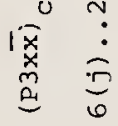

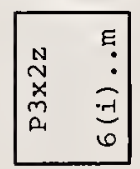

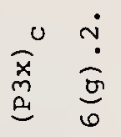

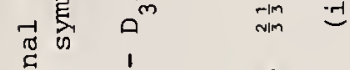

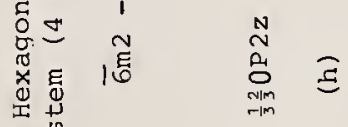

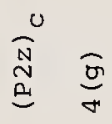

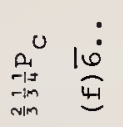

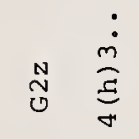

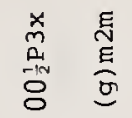

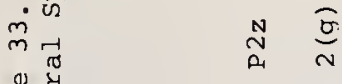

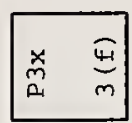

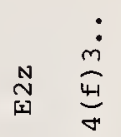

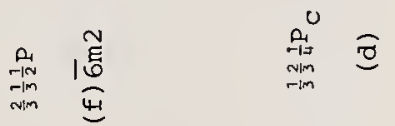

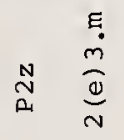

里

$\underset{\substack{\text { 品 } \\ \text { im }}}{\operatorname{lo}}$

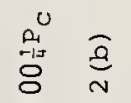

0
0
0
0
0

$m \frac{\widehat{U}}{\mathrm{~N}}$

$\underset{\substack{\text { ind } \\ \text { im }}}{\substack{0 \\ 0}}$

$\stackrel{8}{80}$

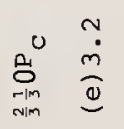

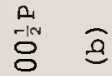

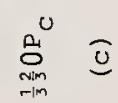

势

$\underset{\substack{0 \\ \stackrel{N}{N}}}{\stackrel{m}{0}}$

a. $\underset{\pi}{\pi}$

$a \frac{\pi}{2}$

a. $\underset{-}{\pi}$

$\operatorname{li}^{0} \underset{\frac{N}{N}}{\stackrel{N}{N}}$

|ี

论

蛋

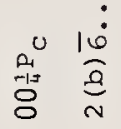

芯 


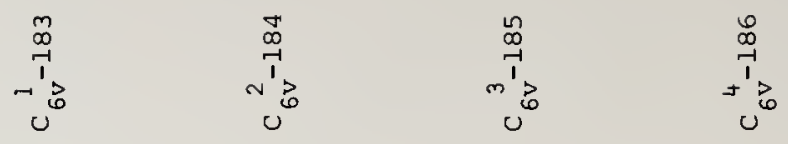

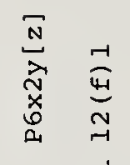

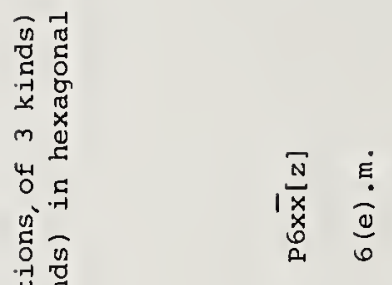

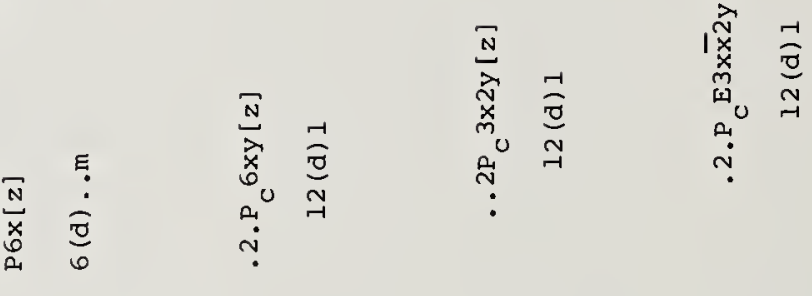

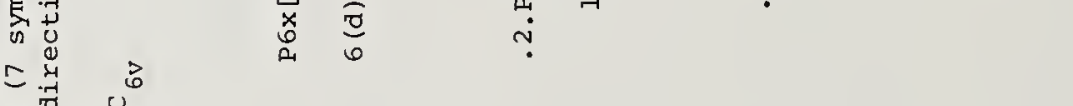

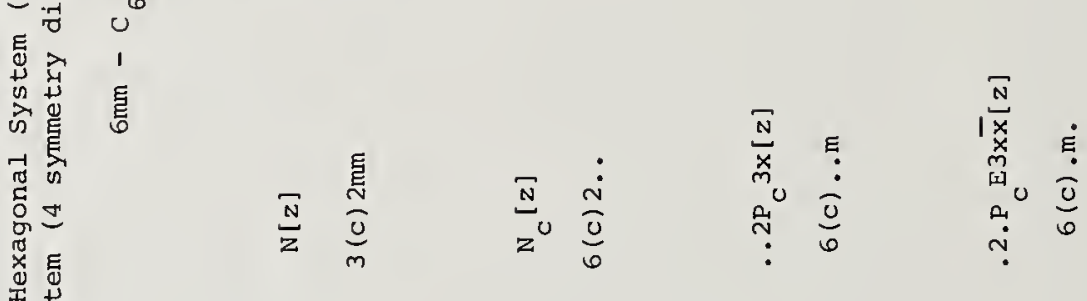

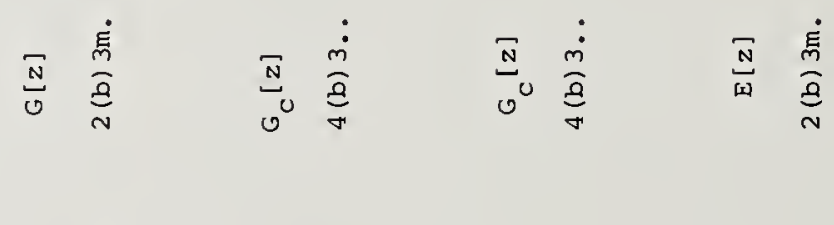

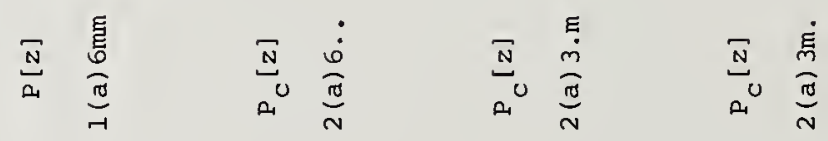

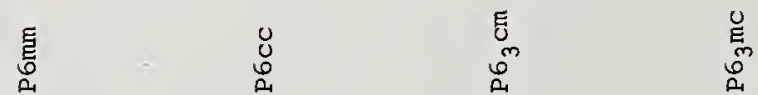




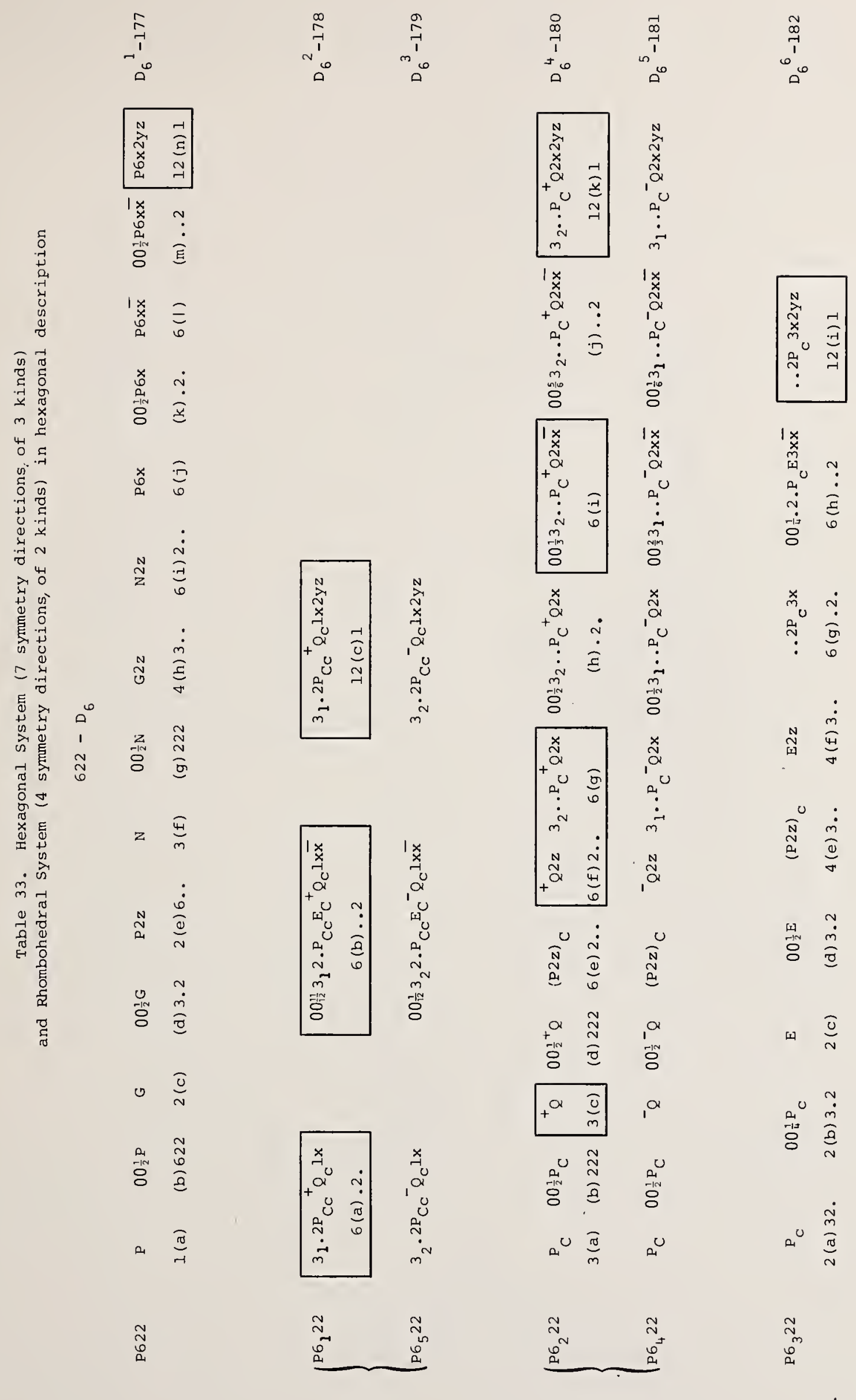




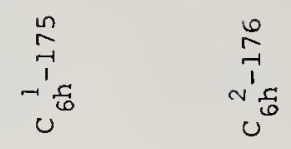

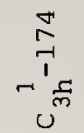
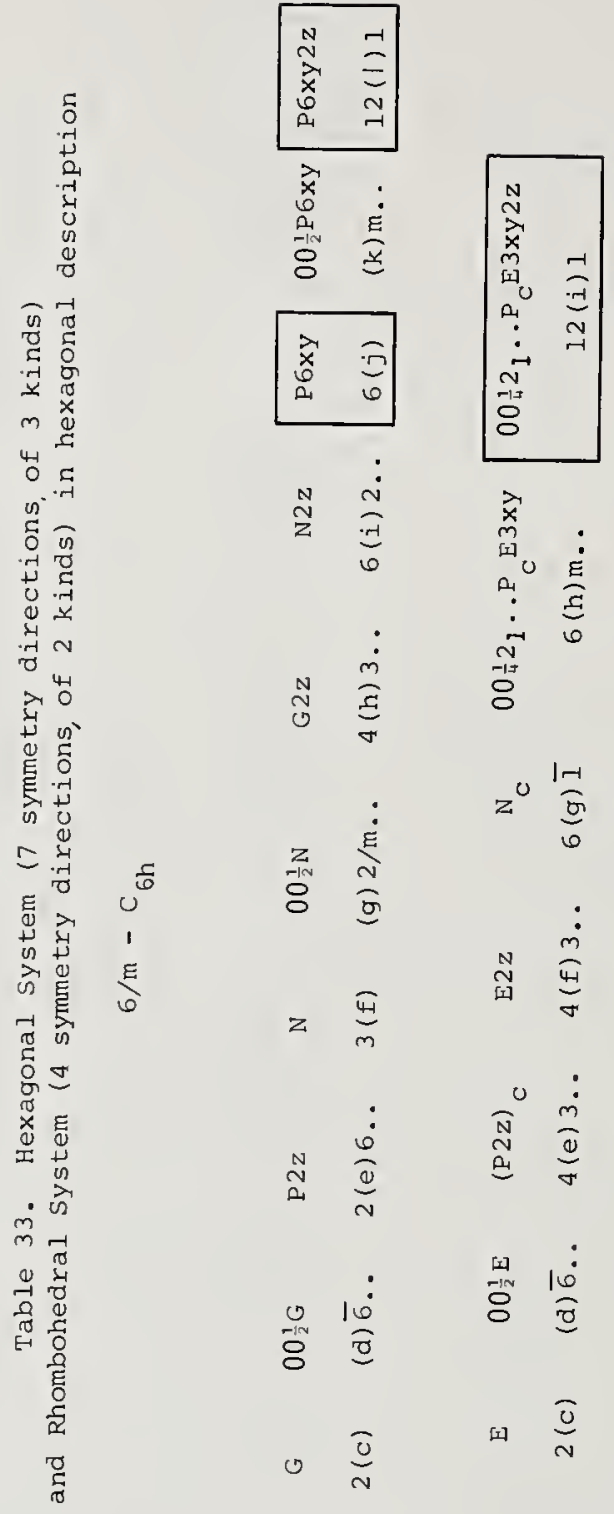

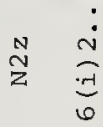

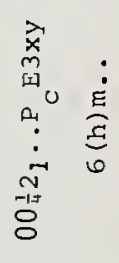

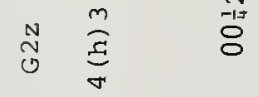

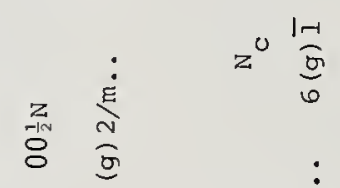

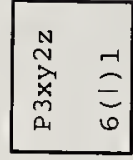

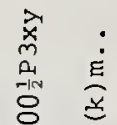

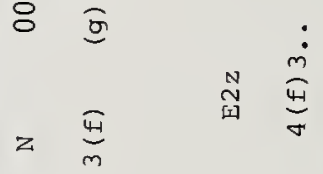

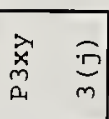

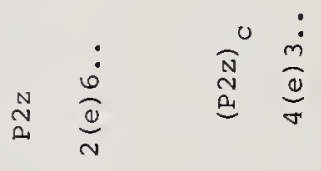

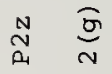

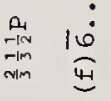

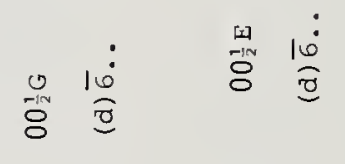

$\underset{\substack{-i m \\ N i m}}{\min }$

$0 \stackrel{0}{\mathrm{~J}}$

m

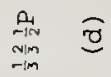

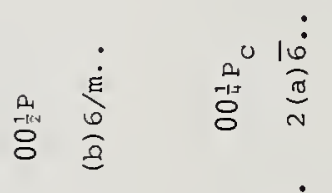

$\operatorname{sim}_{-i m} \hat{0}$

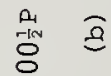

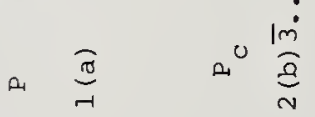

a.

$\underset{1}{\xi}$

$\underbrace{E}_{\substack{m \\ a_{1}}}$

$\log _{01}$ 


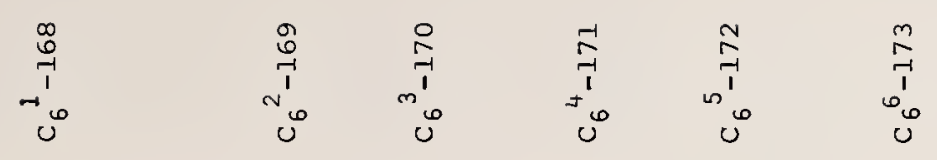

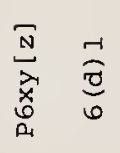

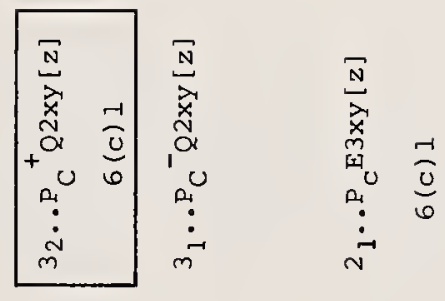

$\underset{0}{\frac{N}{N}} \underset{\sim}{\frac{n}{2}}$

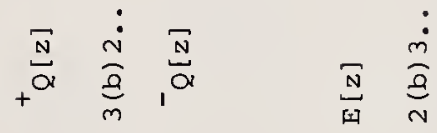

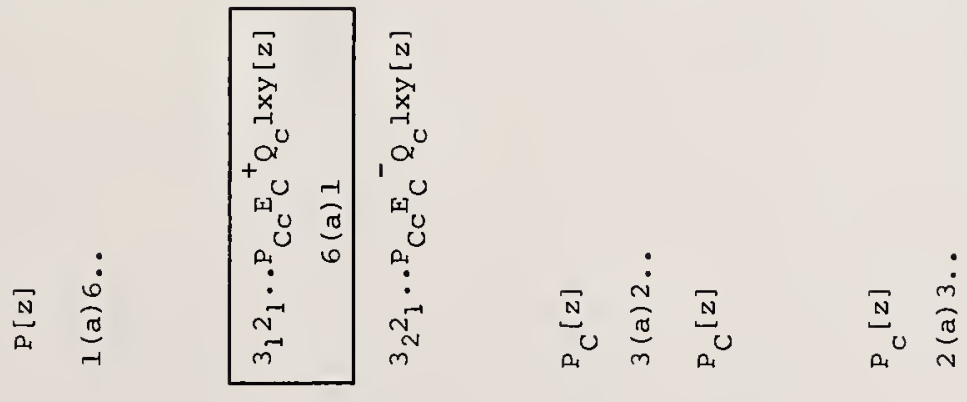

$\stackrel{0}{\circ}$

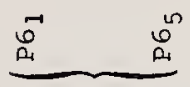

2
0
0

$\stackrel{m}{0}^{m}$ 


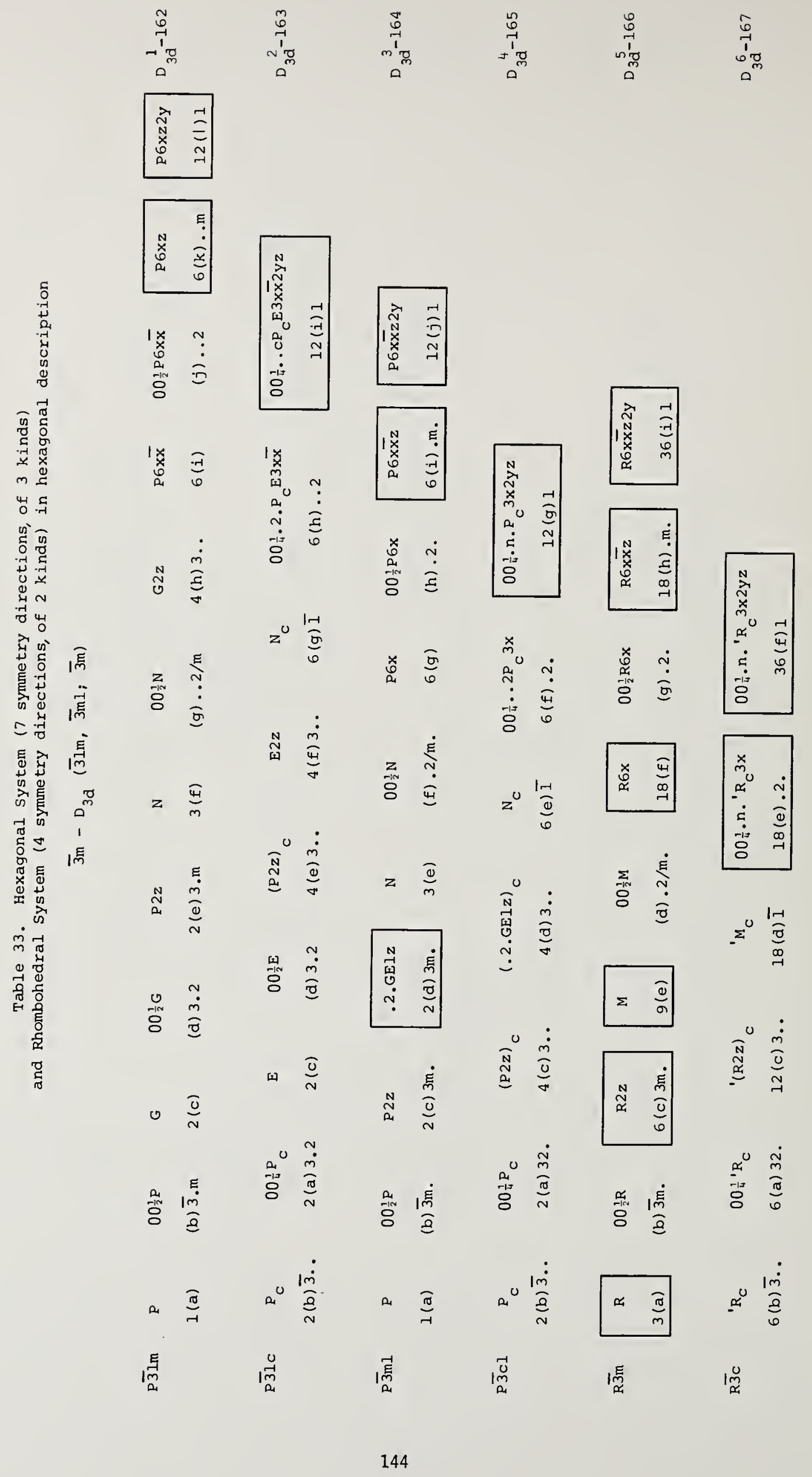




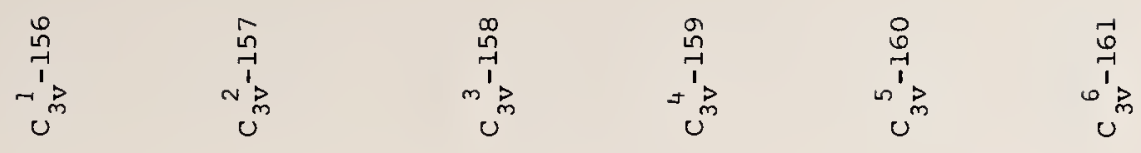

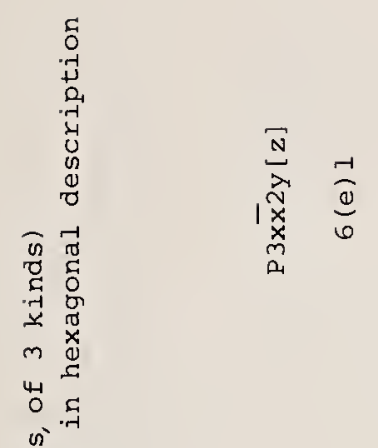

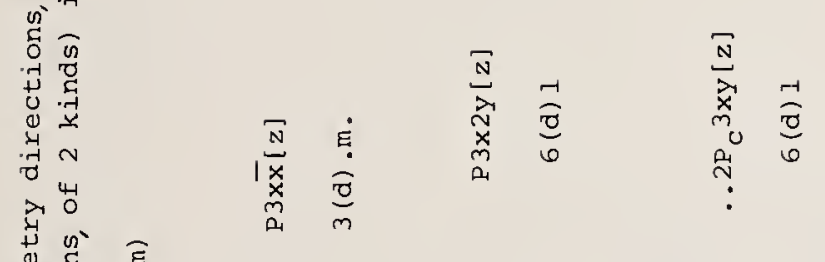

范

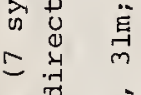

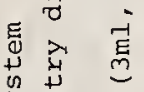

焉

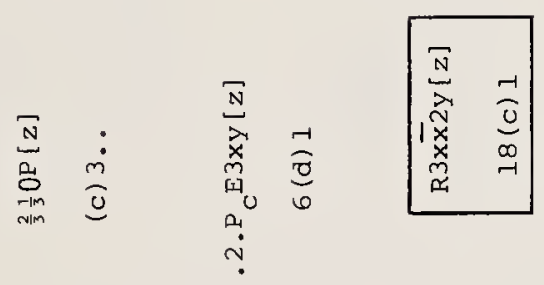

需 $匚$ E

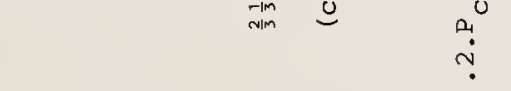

.

ले

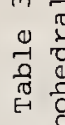

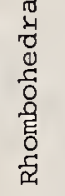

శ్ర

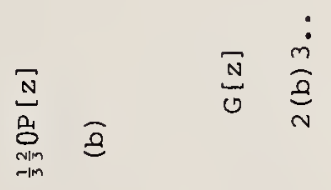

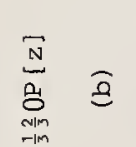

$\underset{\frac{N}{4}}{\stackrel{2}{3}}$

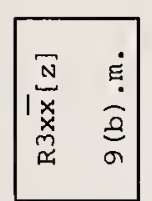

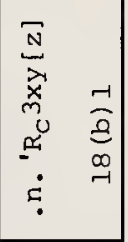

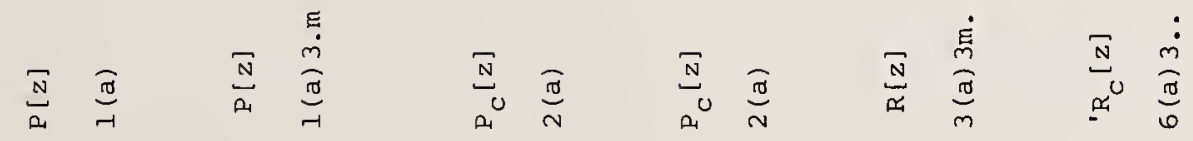

$\stackrel{్}{\mathrm{n}}$

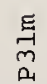

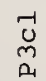

$\underset{0}{0}$

点

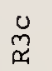


$\frac{a}{a}$

$\stackrel{\substack{0\\}}{N_{m}^{1}}$

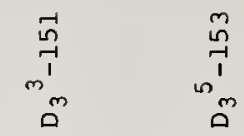

ח

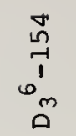

$\overbrace{\substack{n \\ n}}^{n}$

3
3
3
3
3
3

ब्वा

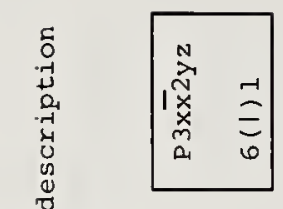

$\frac{0}{-7}$

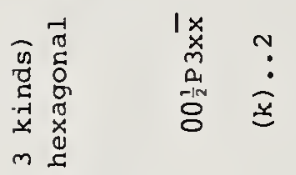

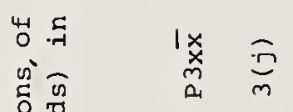

造

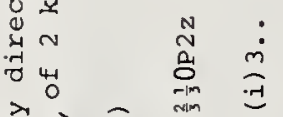

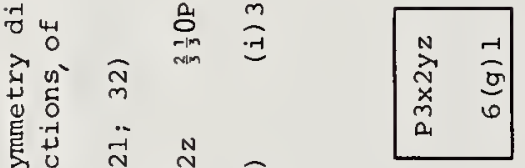

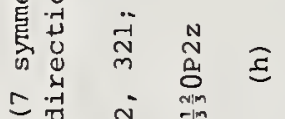

हु

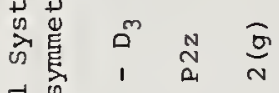

$\begin{array}{ll}\stackrel{x}{2} & \dot{\sim} \\ \substack{-1 \\ 0} & \stackrel{4}{4}\end{array}$

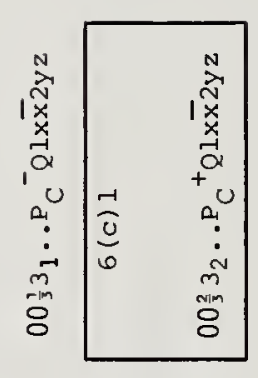

न

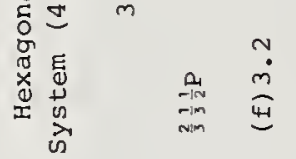

$\stackrel{x}{\stackrel{\infty}{a}}$

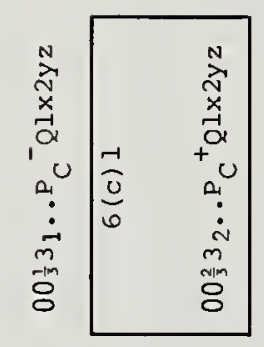

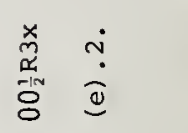

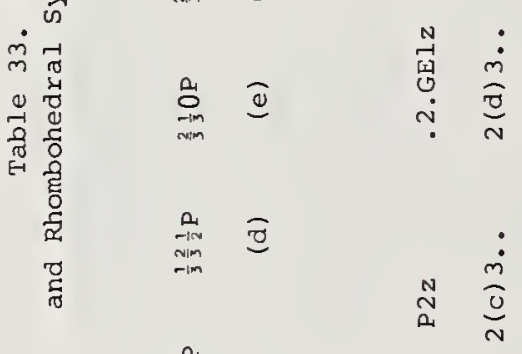

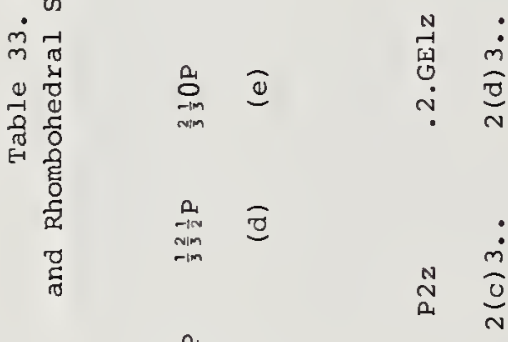

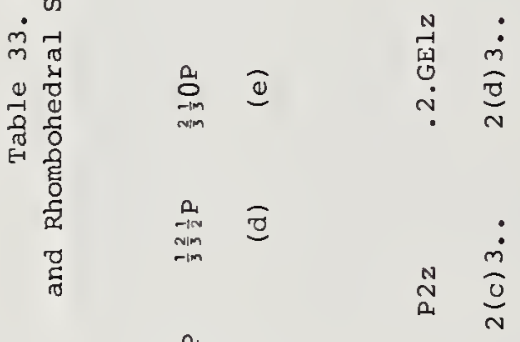

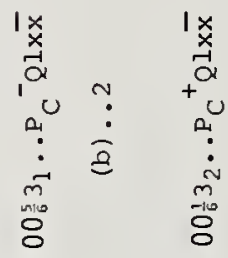

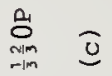

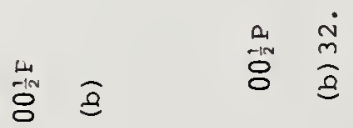

a. $\underbrace{\pi}_{-1}$ a $\frac{\pi}{\pi}$

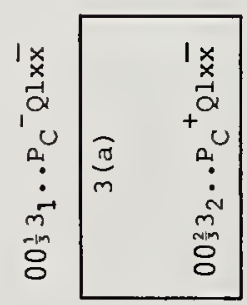

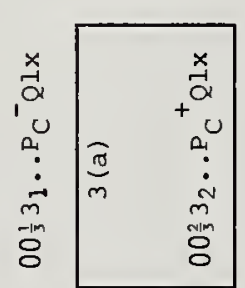

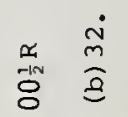

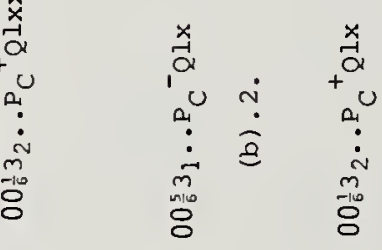

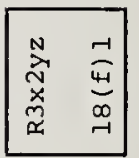

อิ

Tั7

ฮิ।

ก $\frac{\dot{m}}{0}$

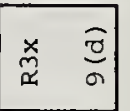

年
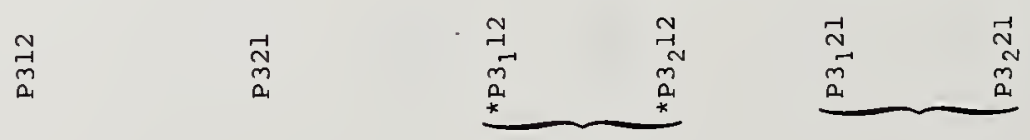

$\pi \frac{\pi}{m}$

ల్జ

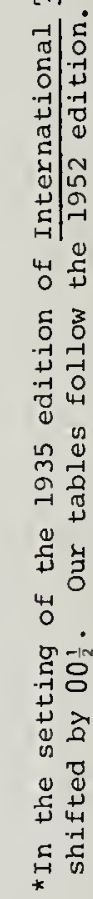




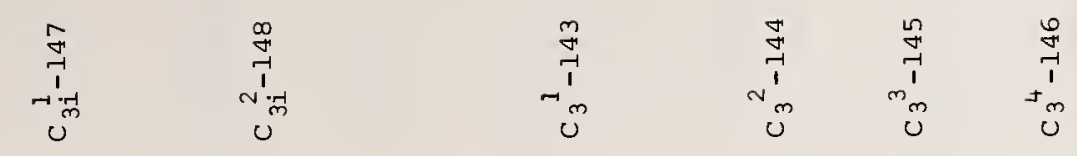

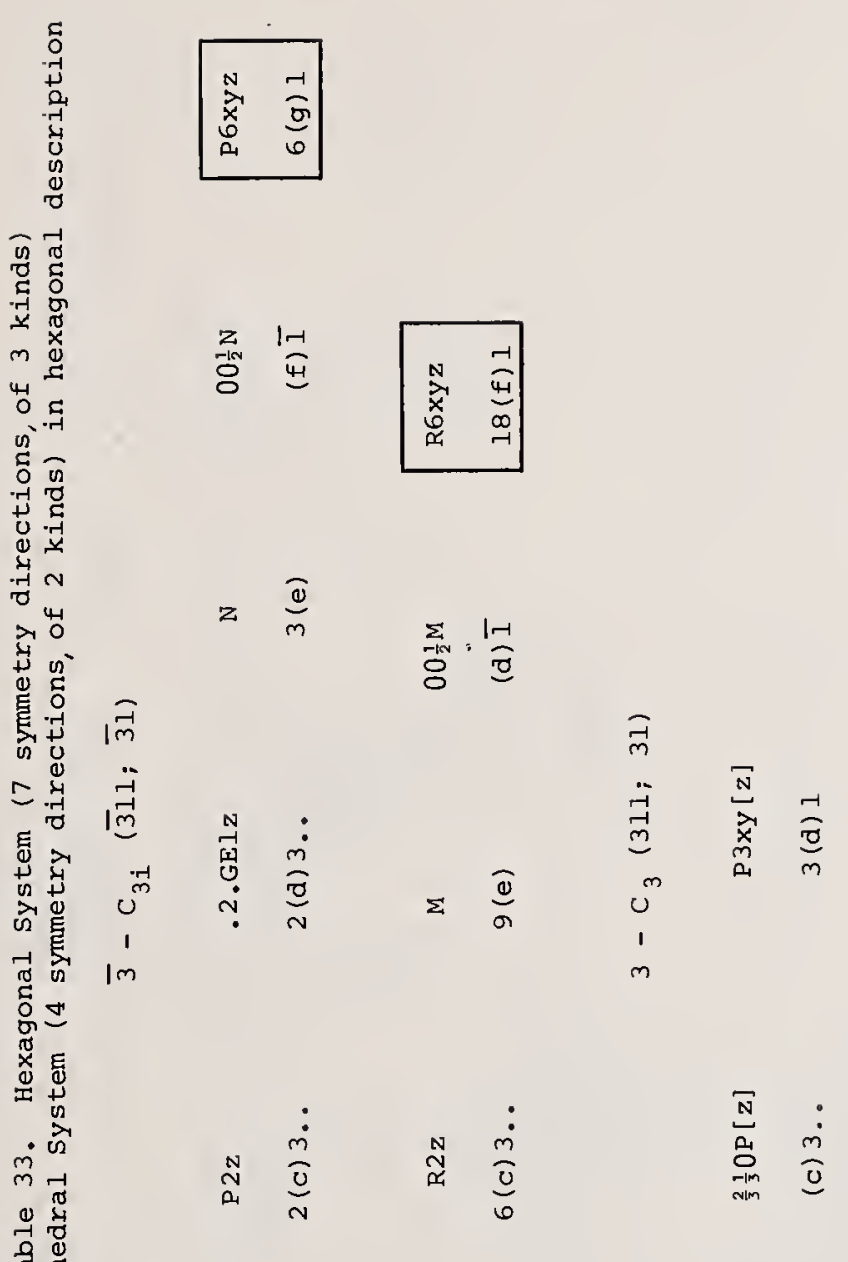

疍.

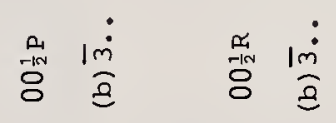
$\underset{\substack{\operatorname{sim} \\ \text { am }}}{\stackrel{\mathbb{N}}{a}} \mathfrak{a}$
a. $\underset{\frac{\pi}{\pi}}{\pi} \frac{\pi}{0}$

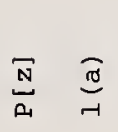

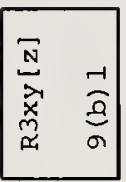

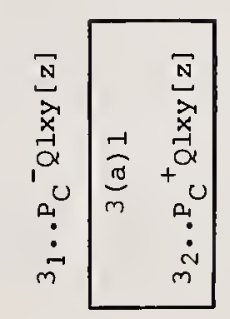
$\underset{\sim}{\stackrel{N}{*}} \frac{\dot{m}}{m}$

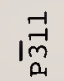
$\operatorname{lm}_{\mathscr{x}}$
$\underset{m}{71}$

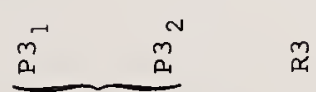




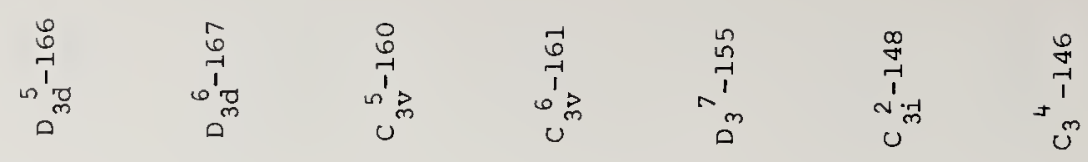

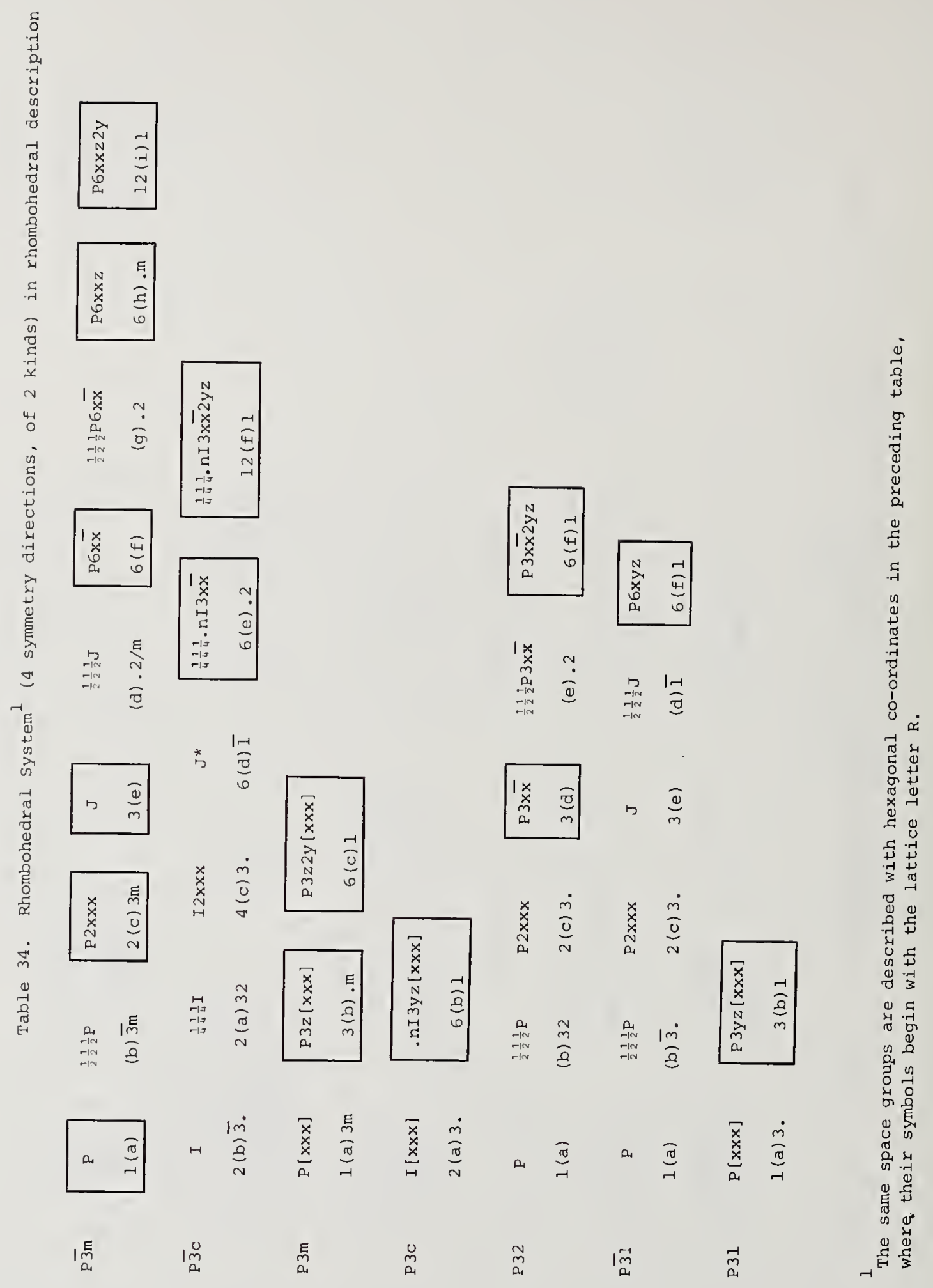


$\frac{\pi}{3}$

$\frac{\sqrt{2}}{\sqrt[3]{2}}$

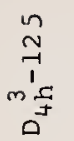

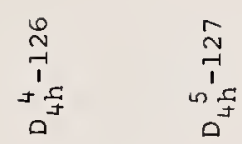

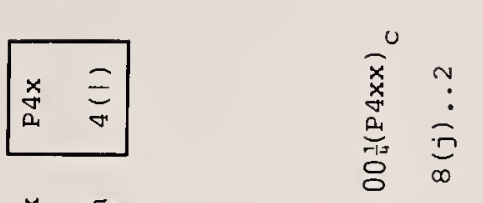

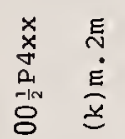

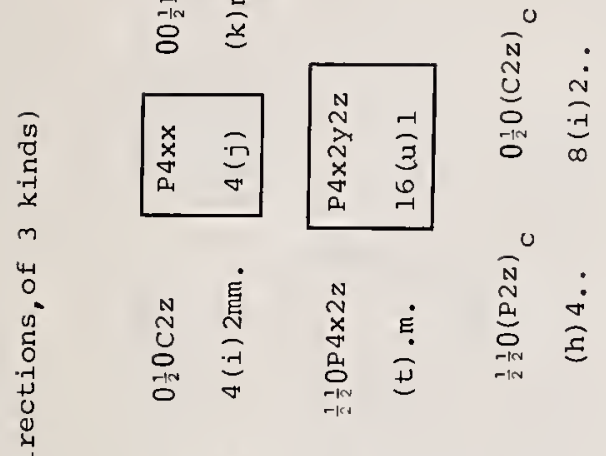

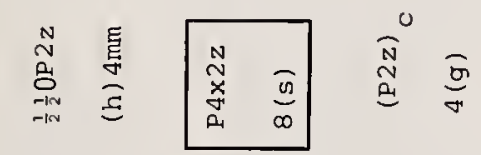

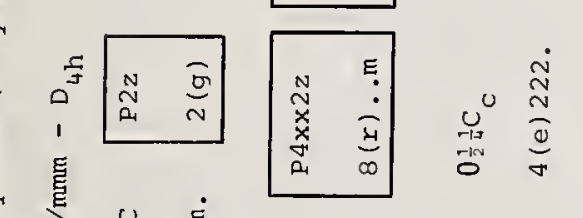

임

놓훙

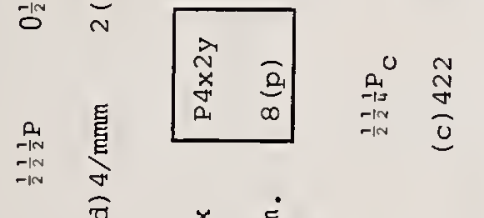

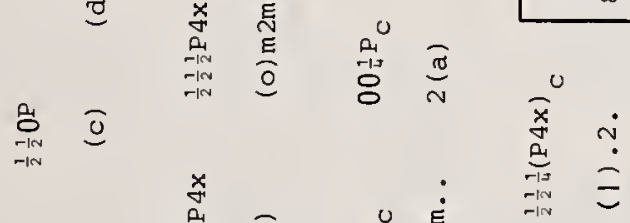

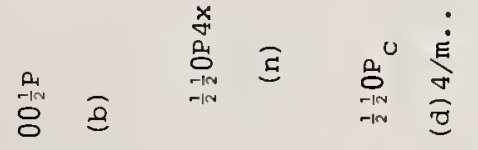

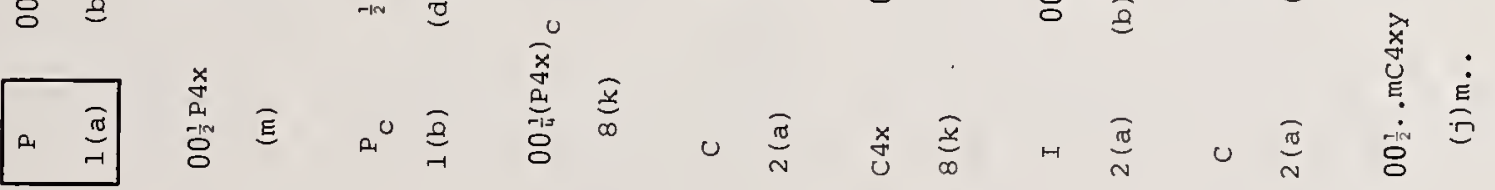

I
衰
部辛 


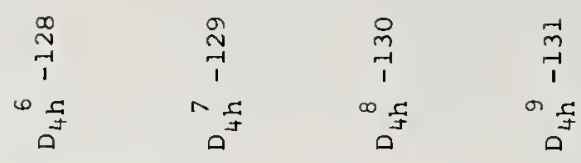

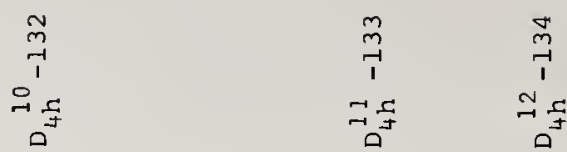

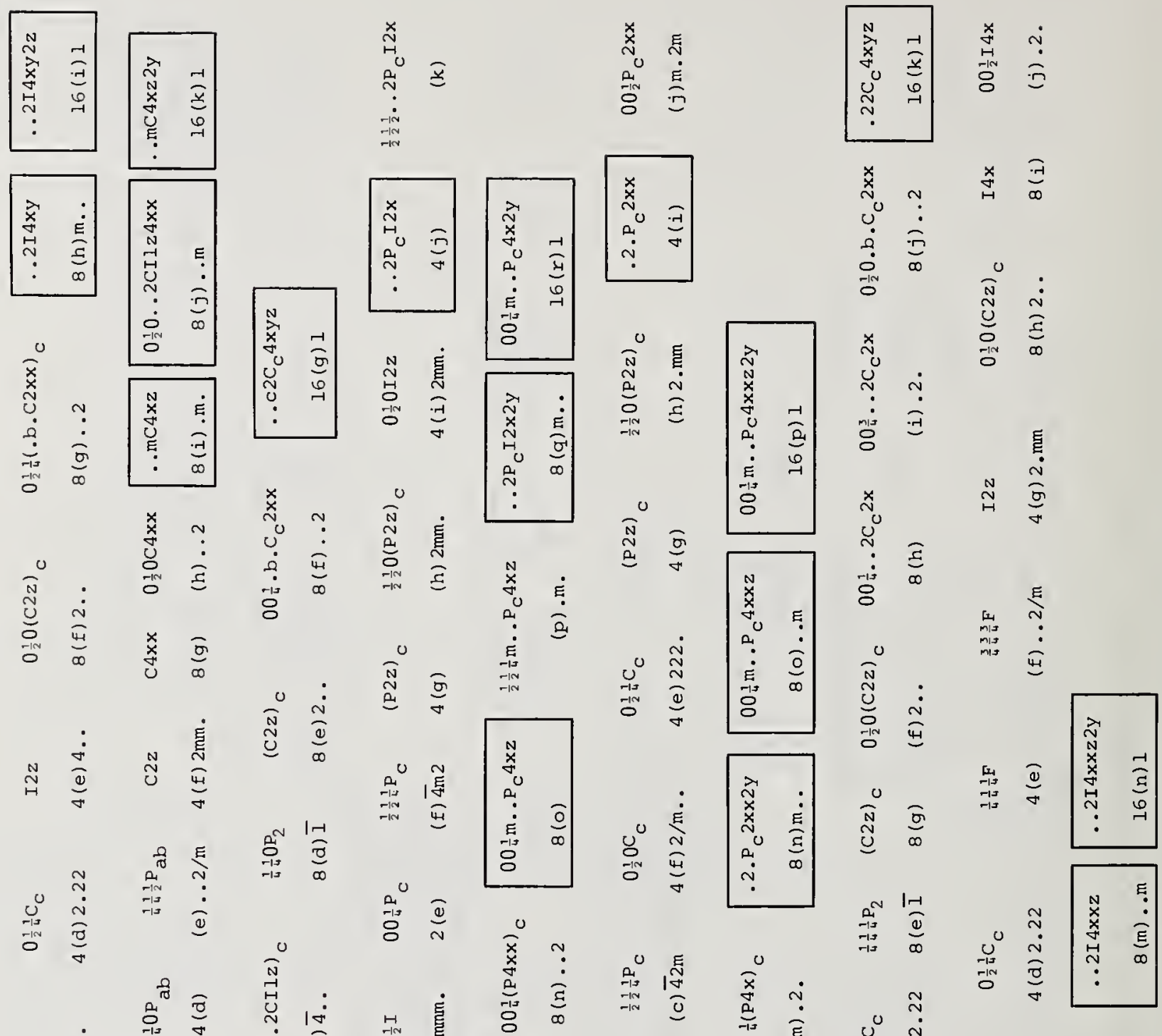

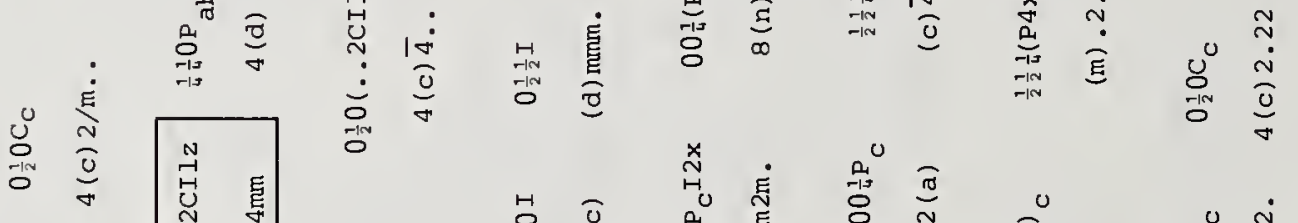

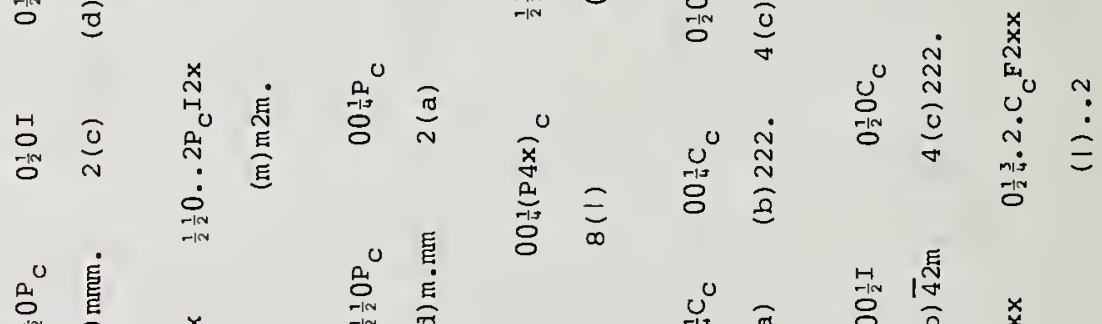

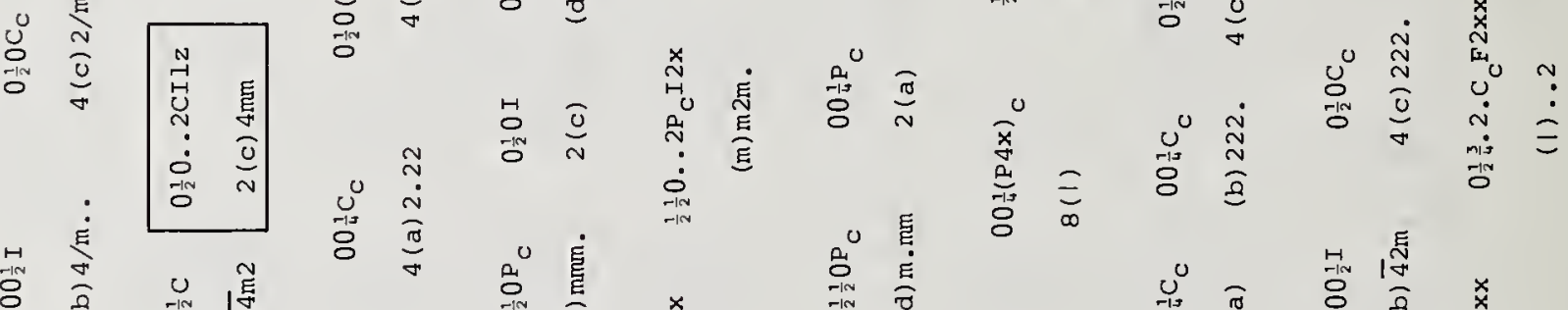

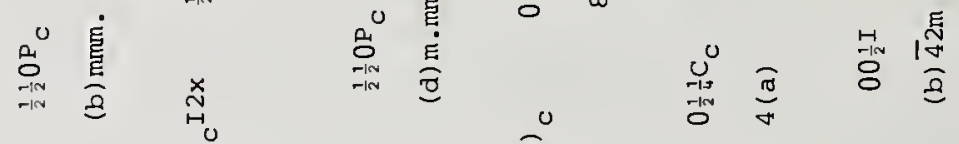

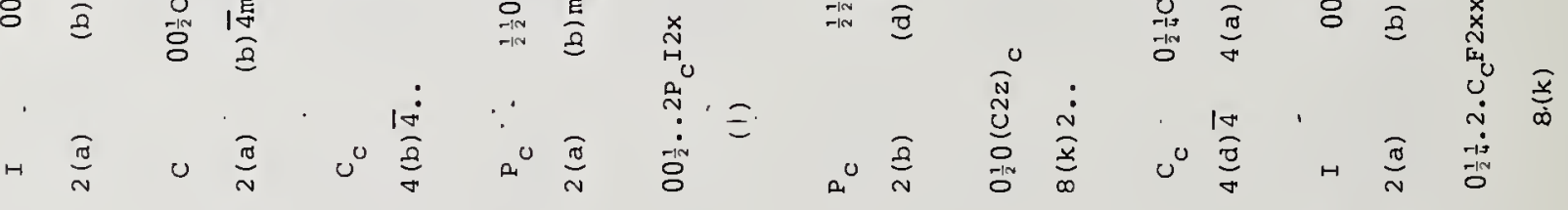

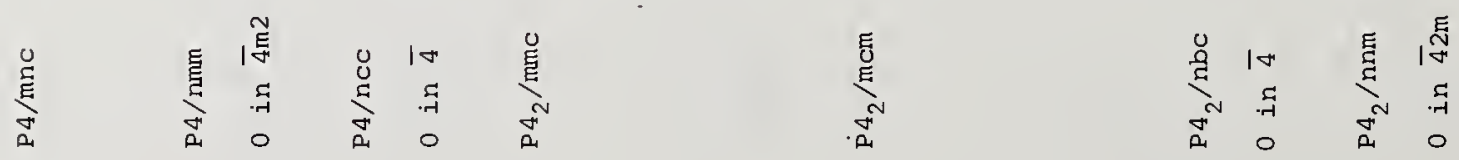




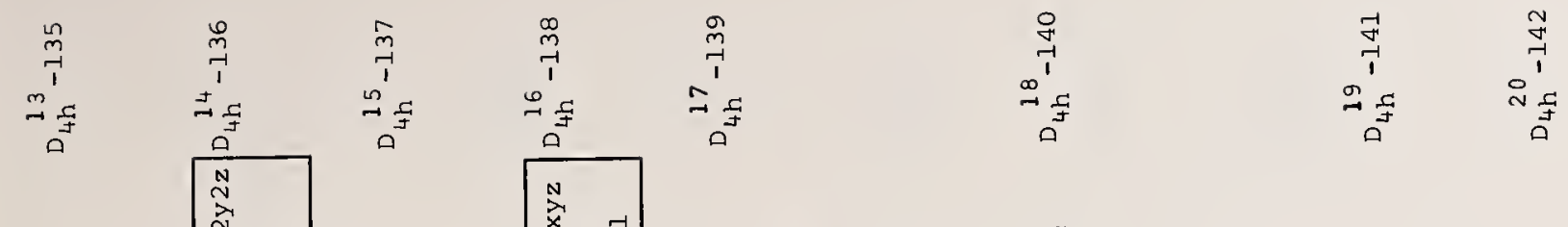

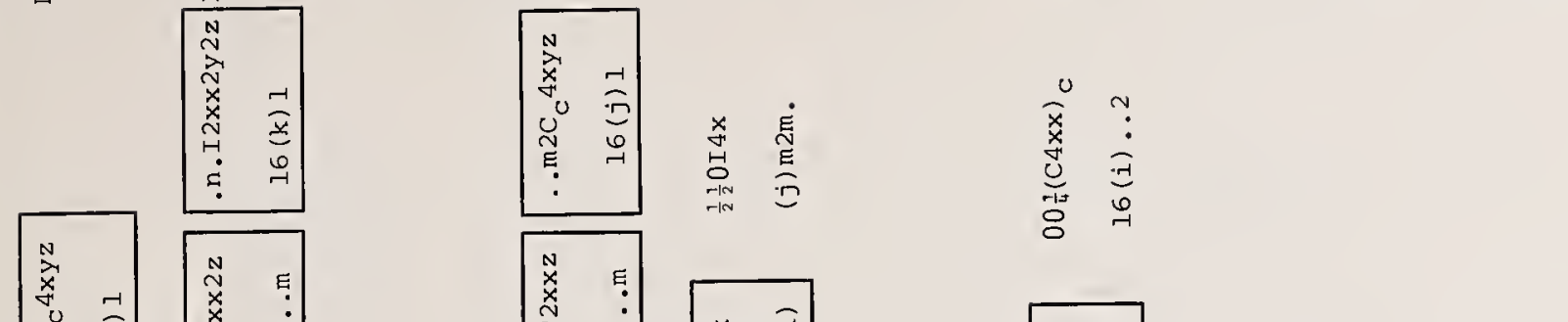

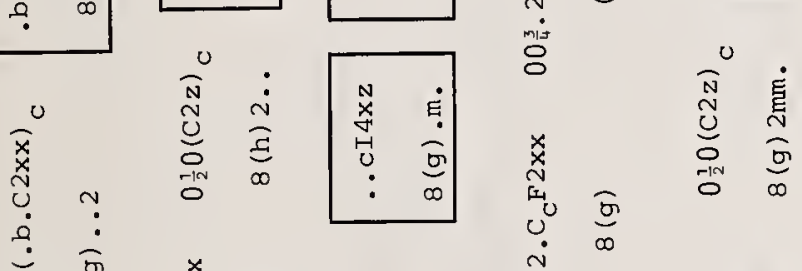

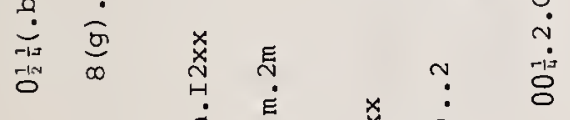

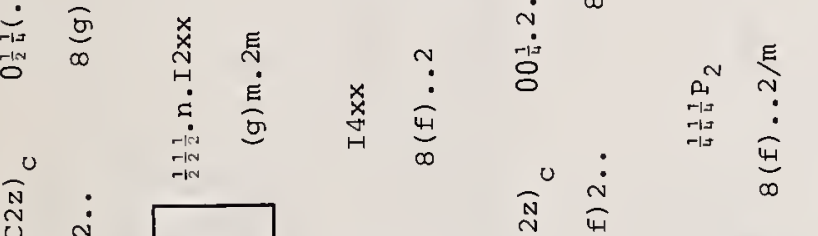

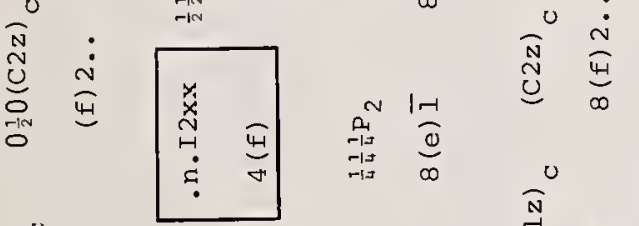

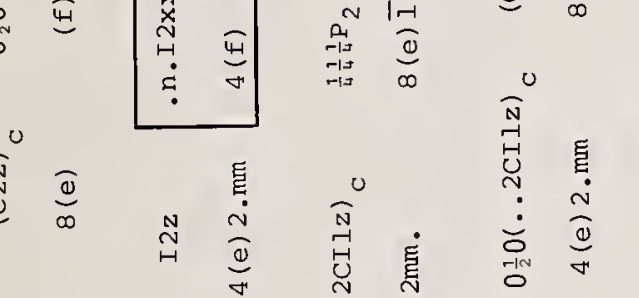

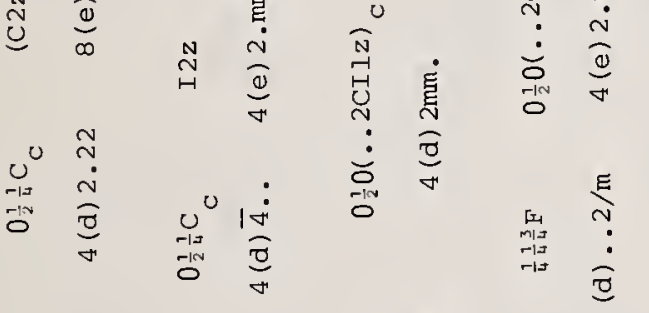

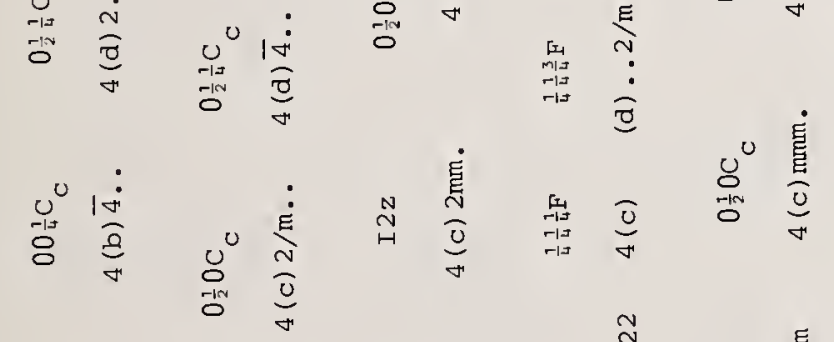

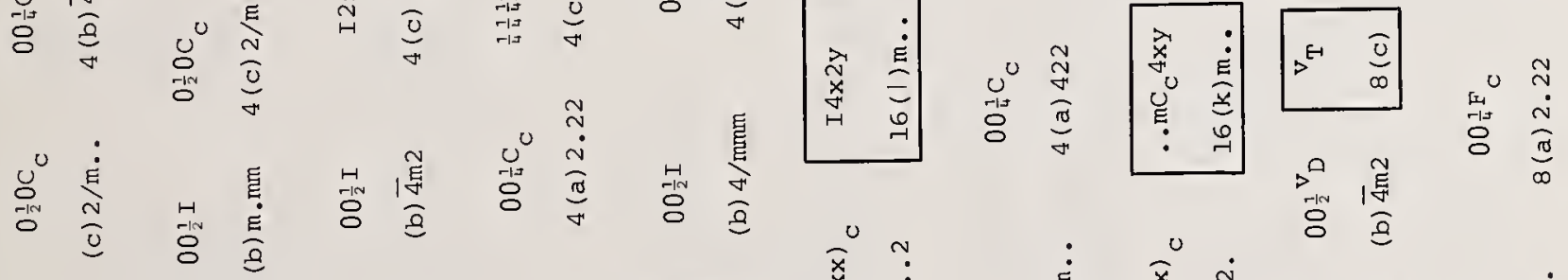

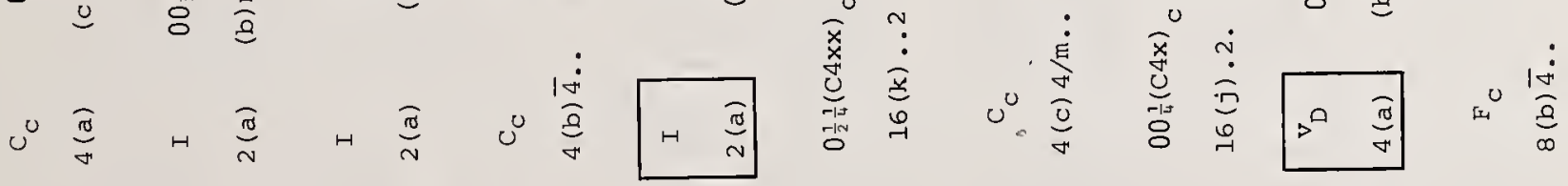

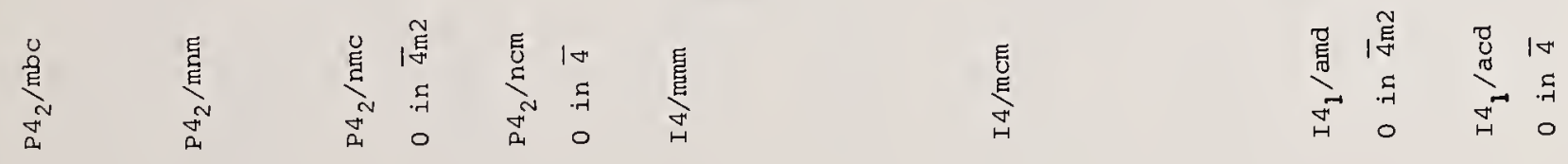




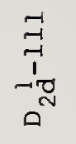

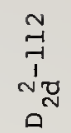

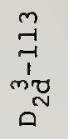

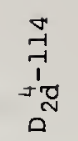

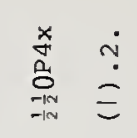

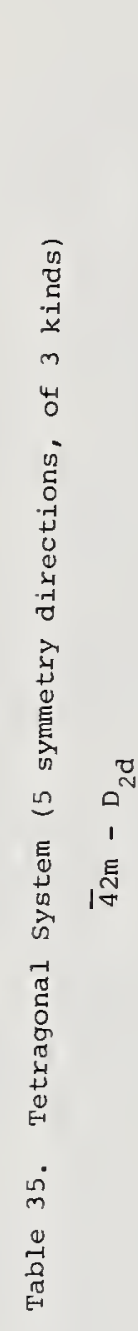

$\underset{\substack { x \\ \begin{subarray}{c}{0 \\-1{ x \\ \begin{subarray} { c } { 0 \\ - 1 } } \\{0} \\{0}\end{subarray}}{\substack{x \\ 0}}$

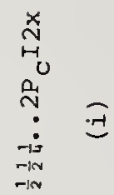

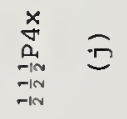

$\underset{0}{x} \stackrel{x}{\frac{\pi}{4}}$

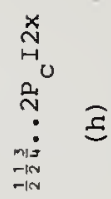

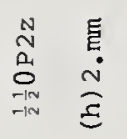

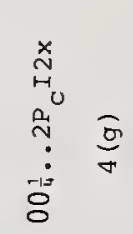

N

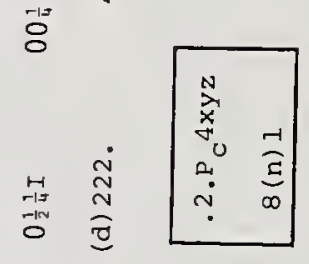

落

$\underset{-1 \times}{\stackrel{0}{N}}$

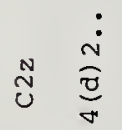

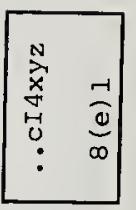

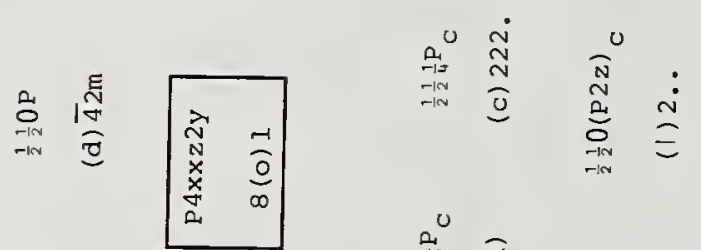

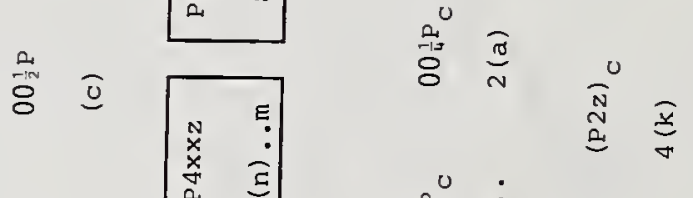

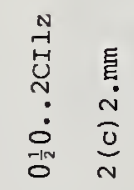

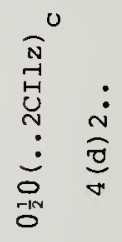

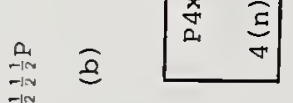

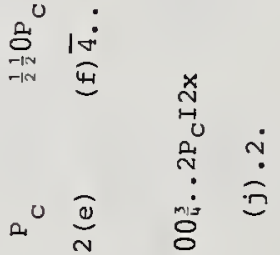

要 $\frac{10}{3}$

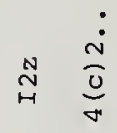

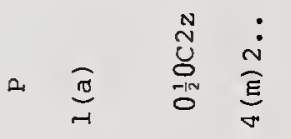

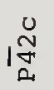

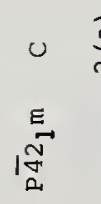

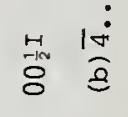

唇

$\underset{I}{E}$

音 


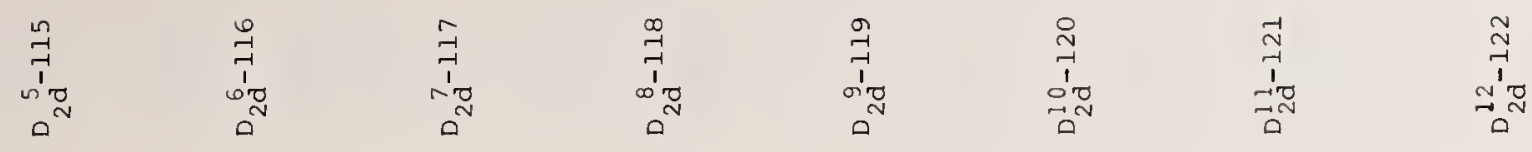
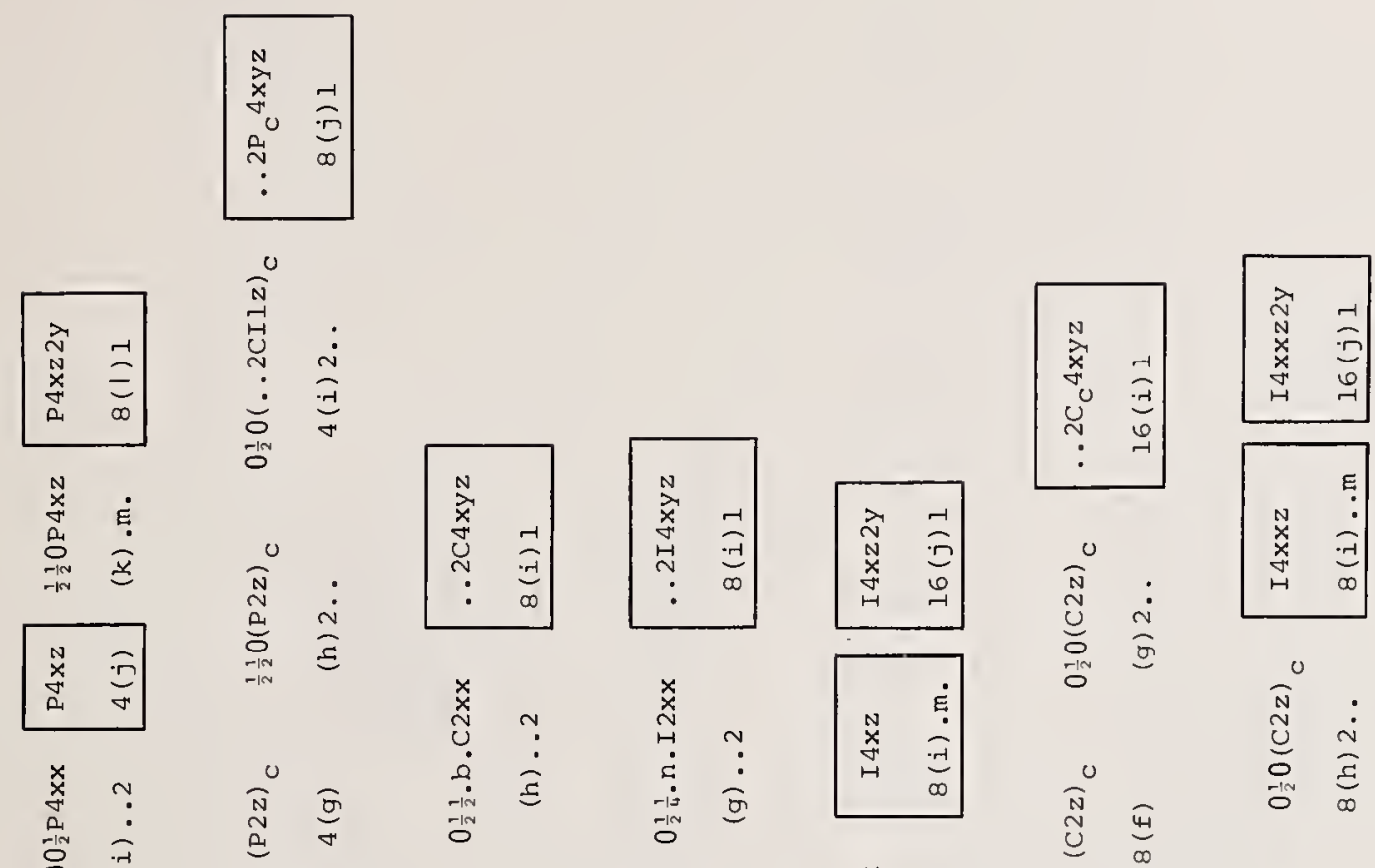

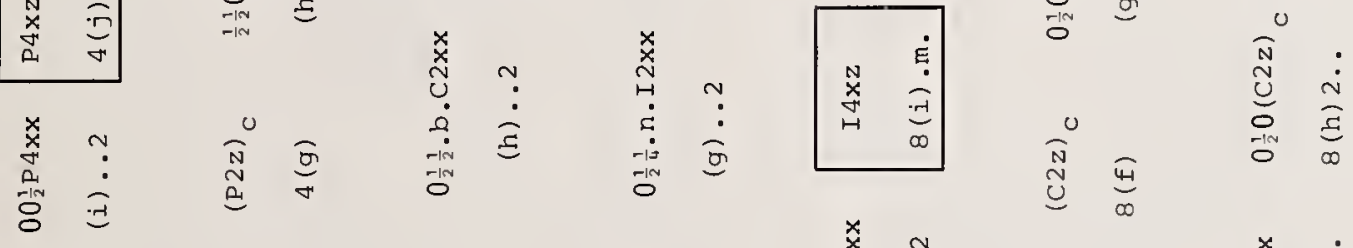

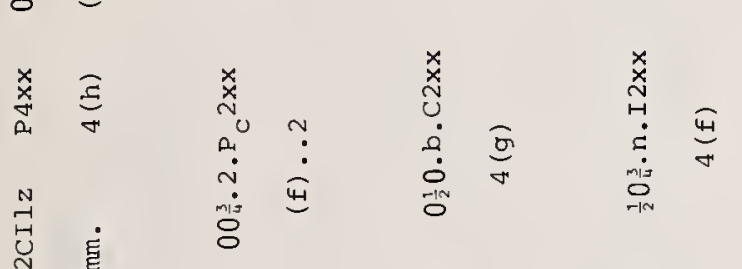

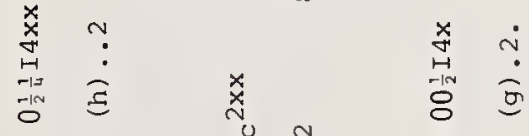

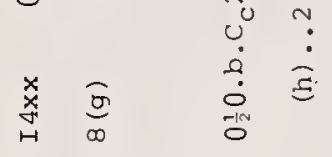

苦产

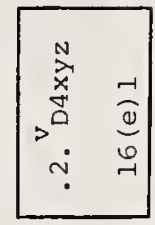

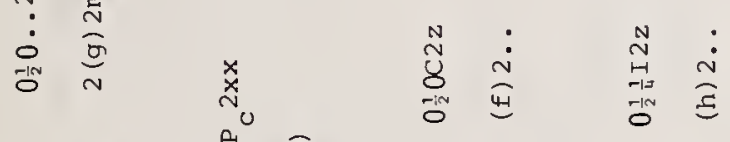

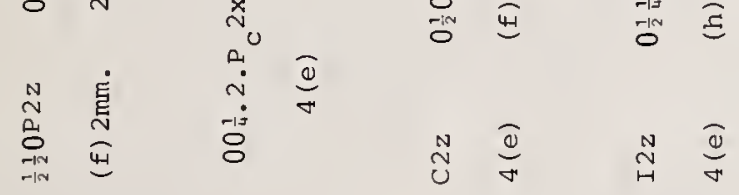

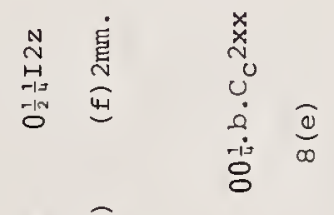

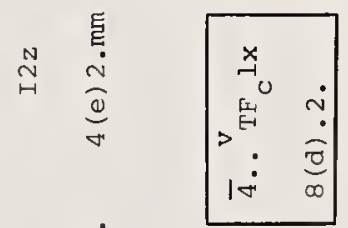

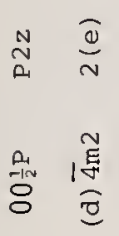

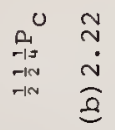

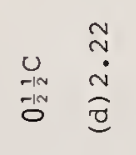

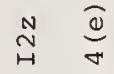

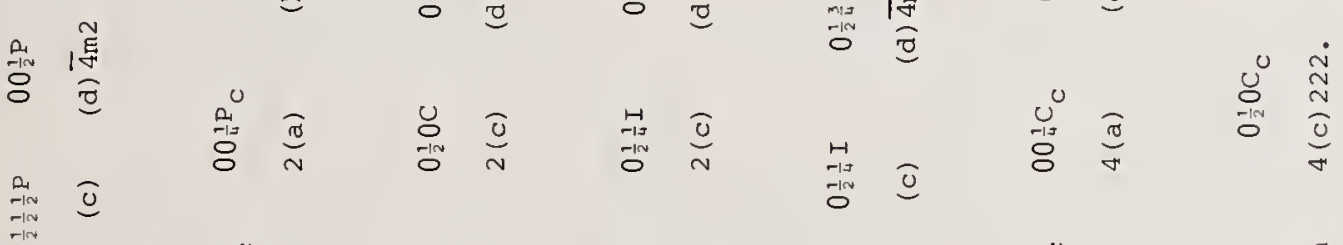

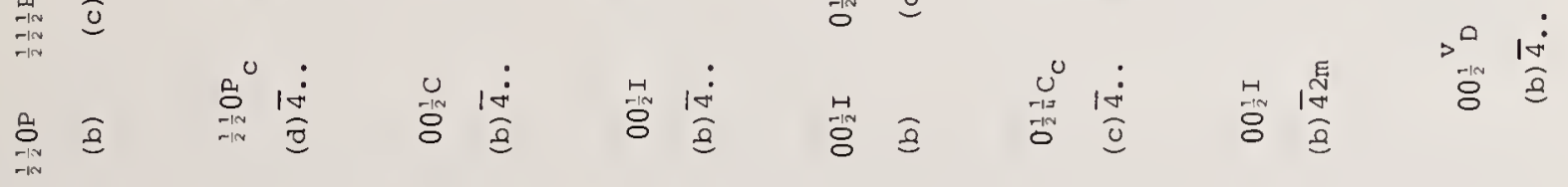

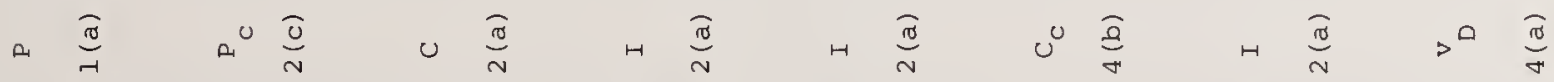

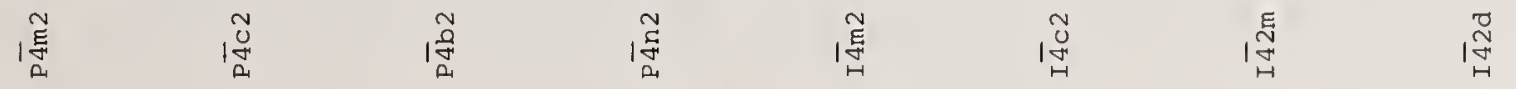




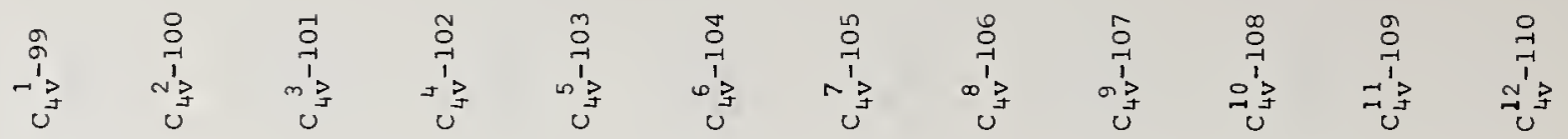

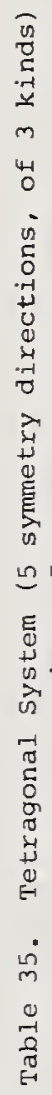

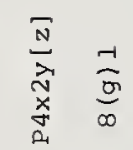

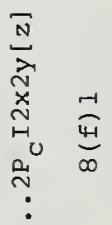

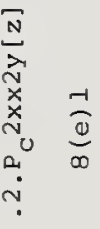

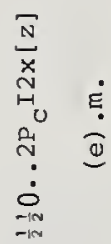

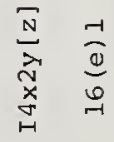

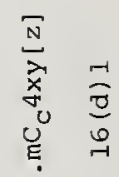

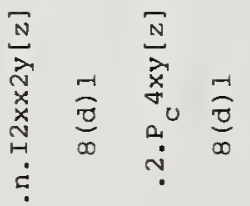

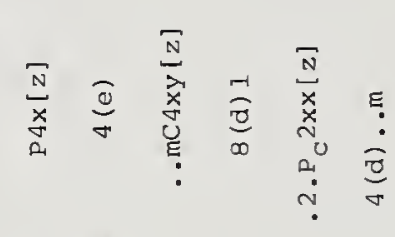

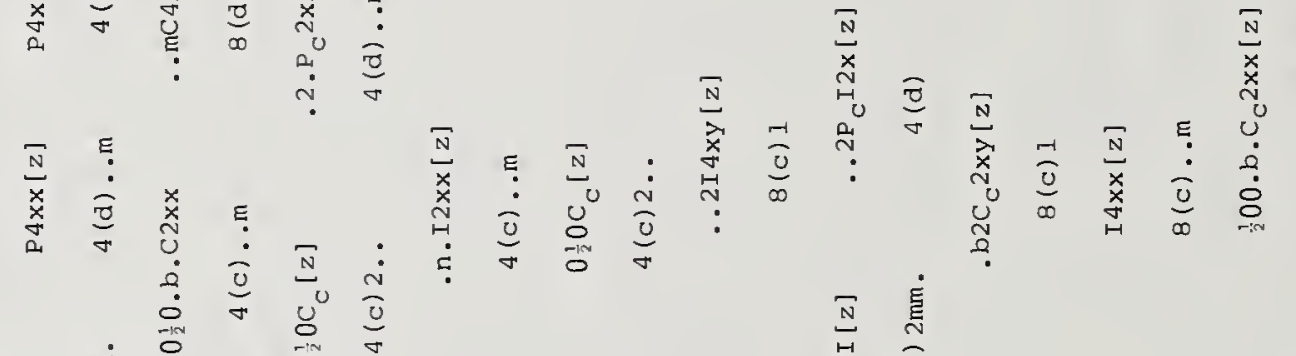

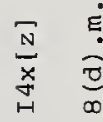

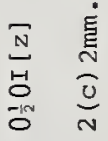

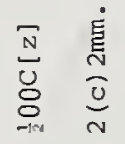

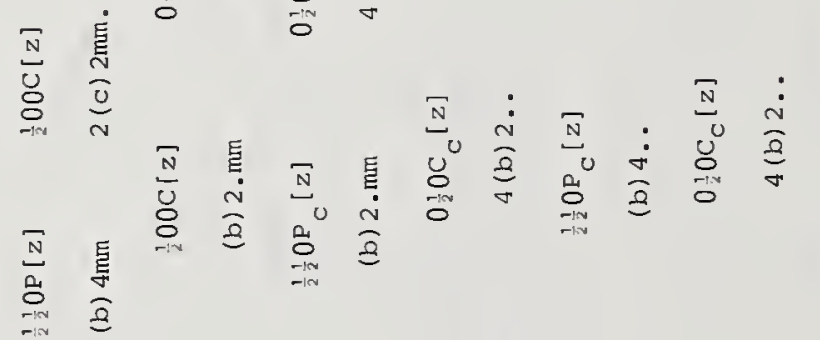

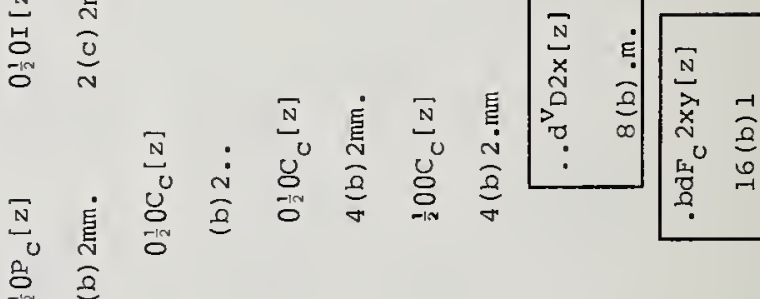

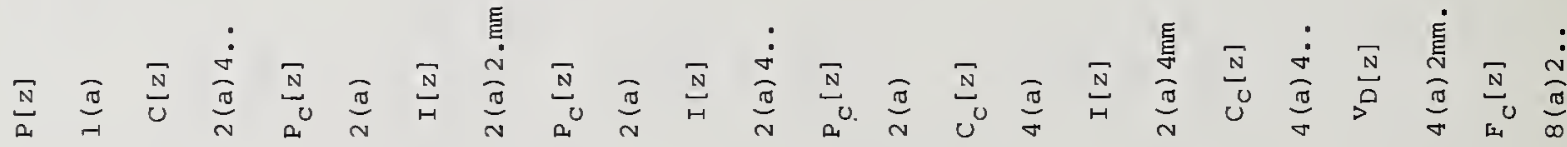

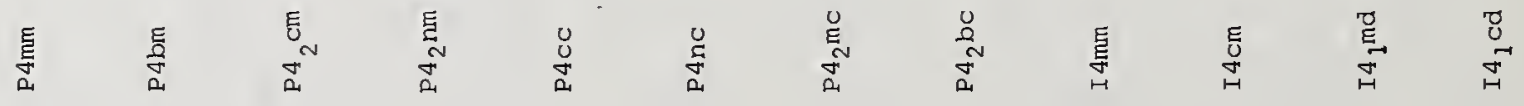




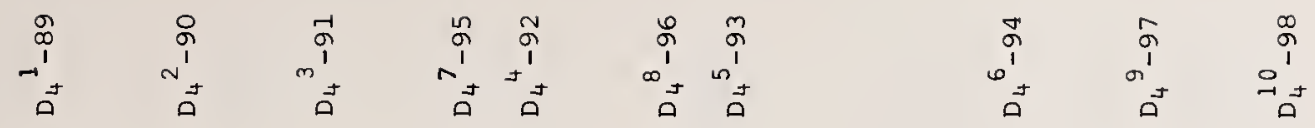

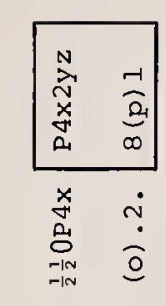

齐

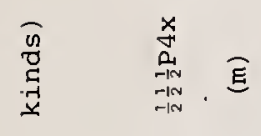

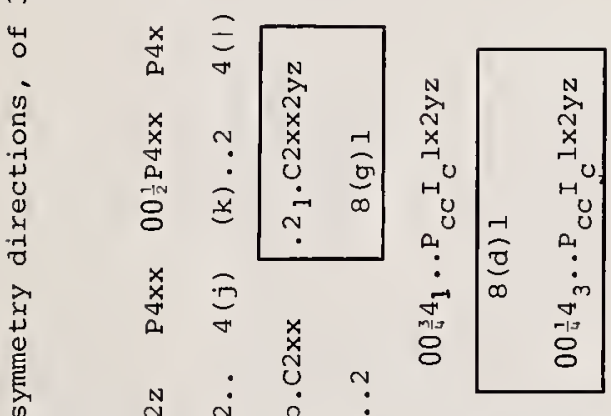

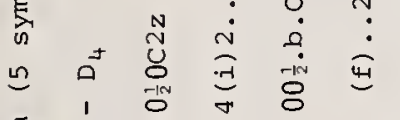

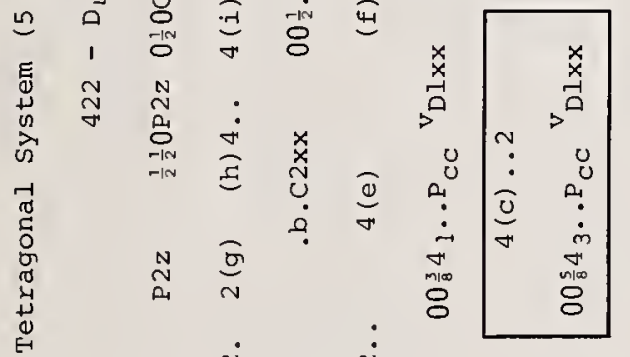

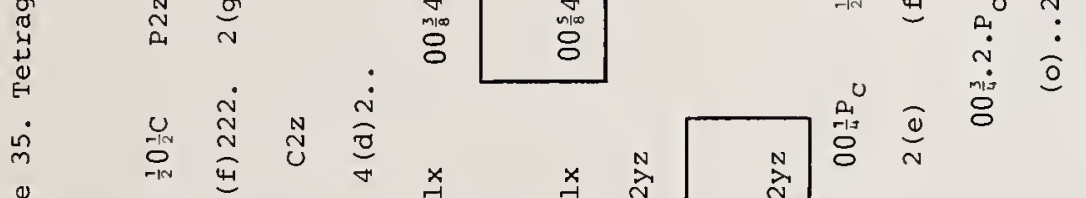

疍

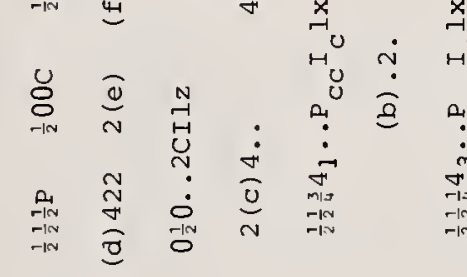

总 0

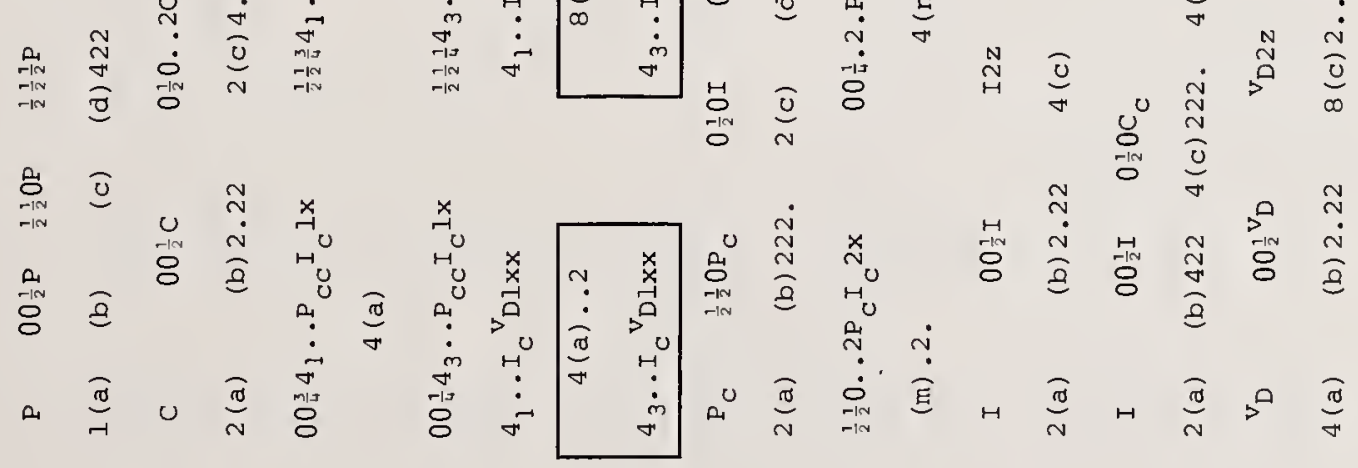

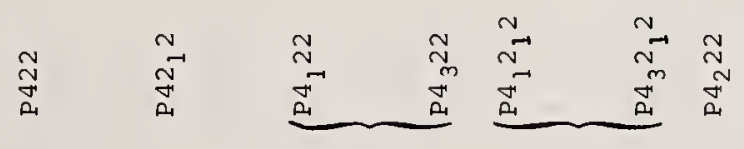

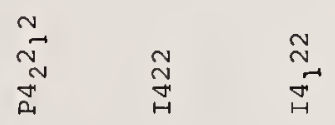




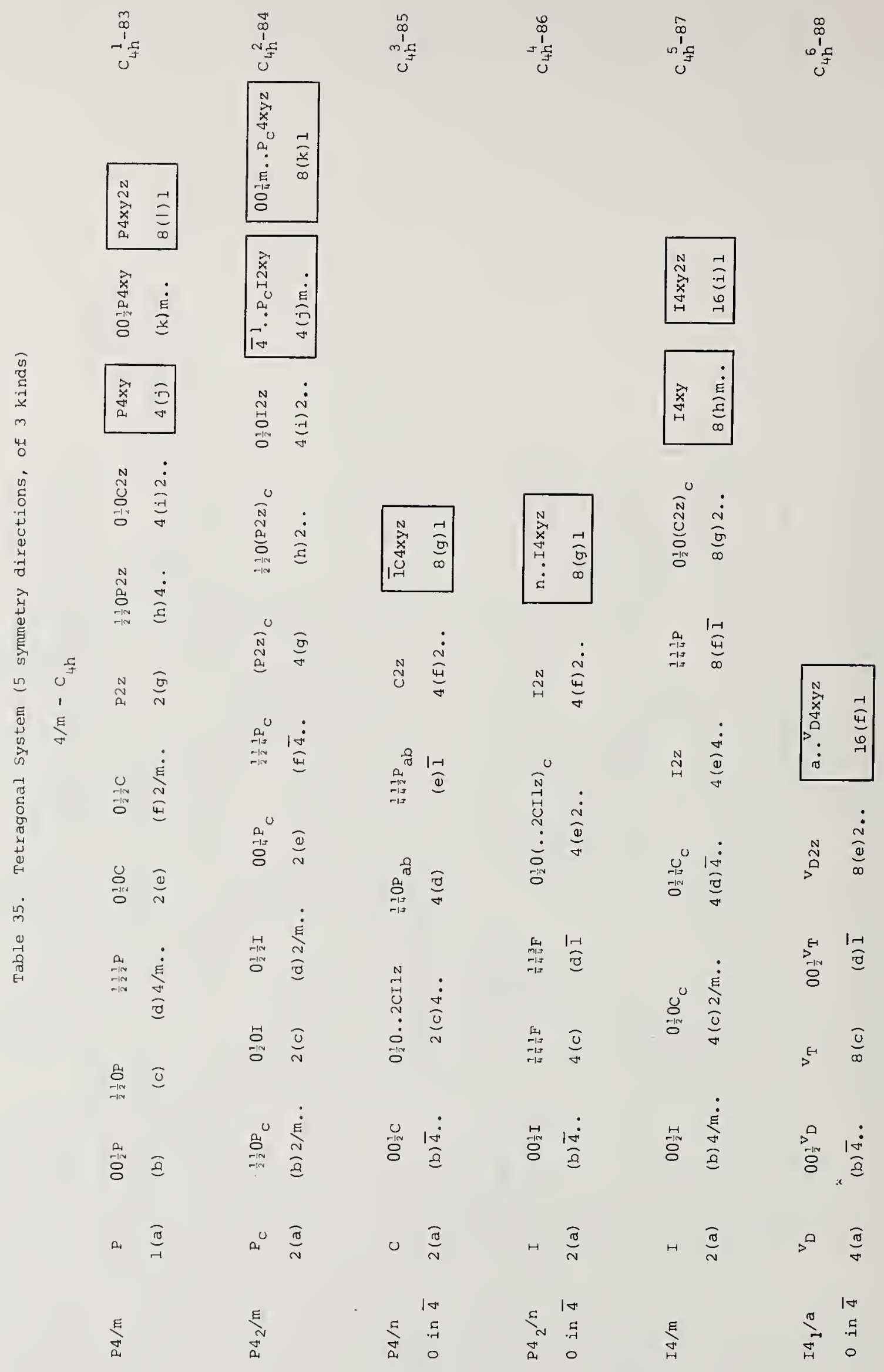




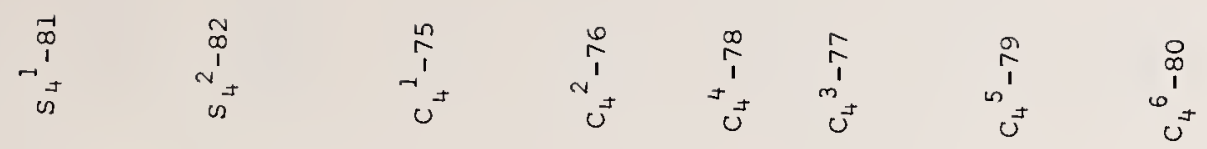

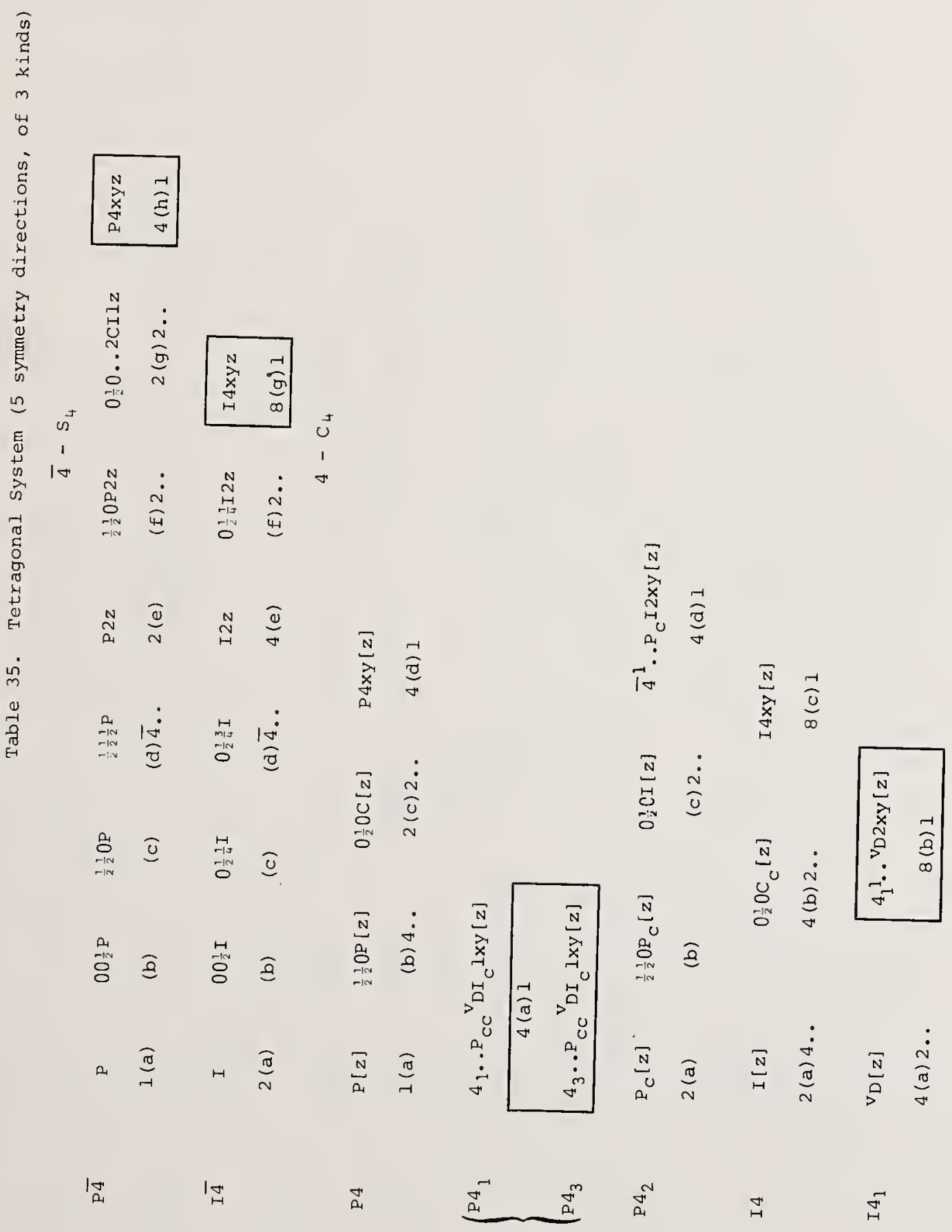




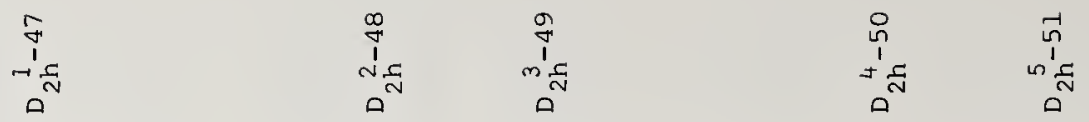

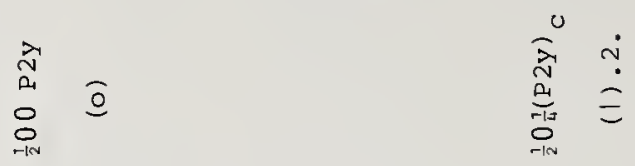

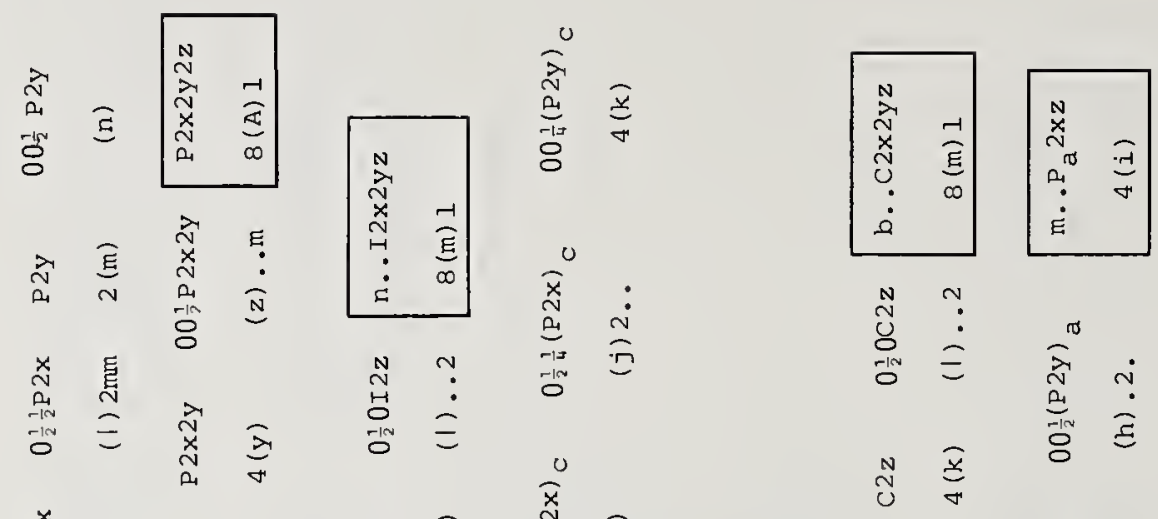

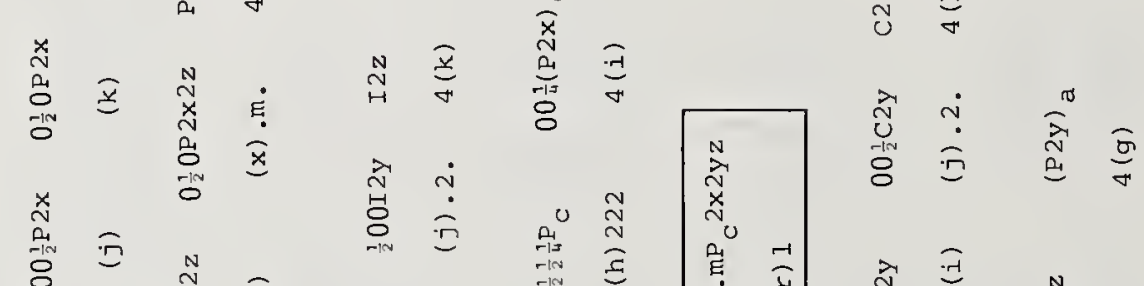

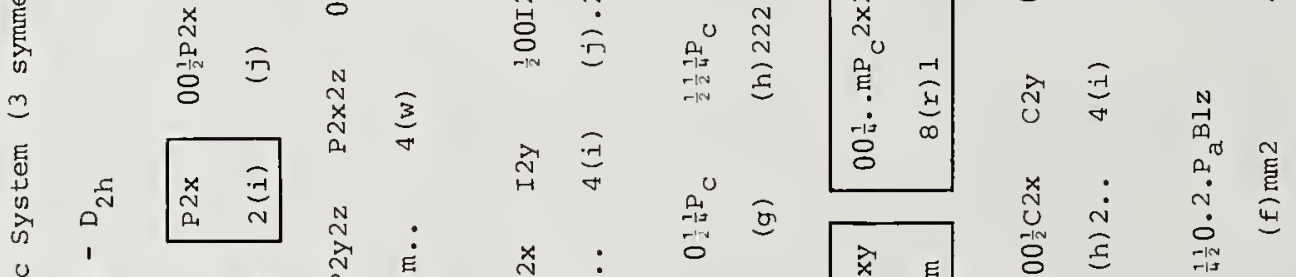

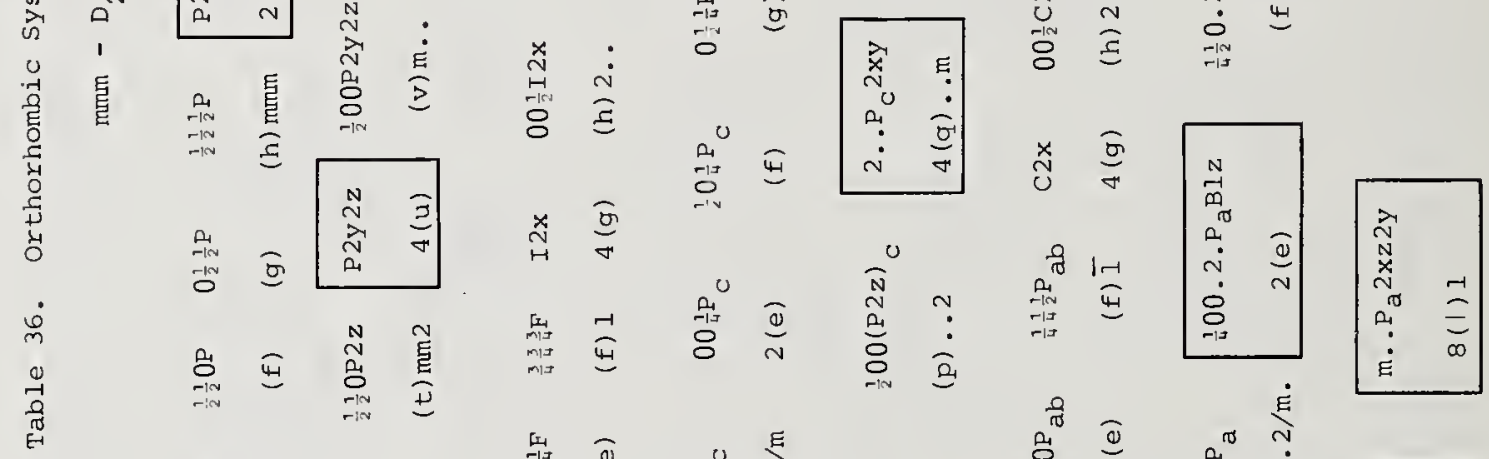

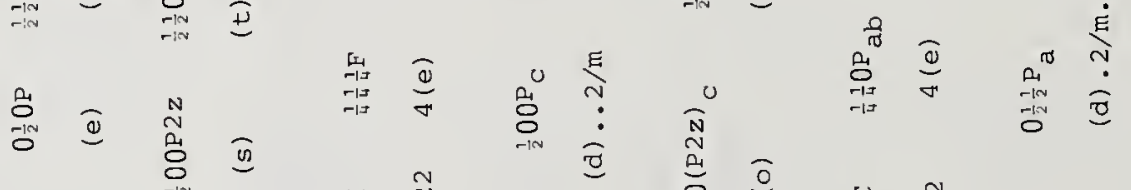

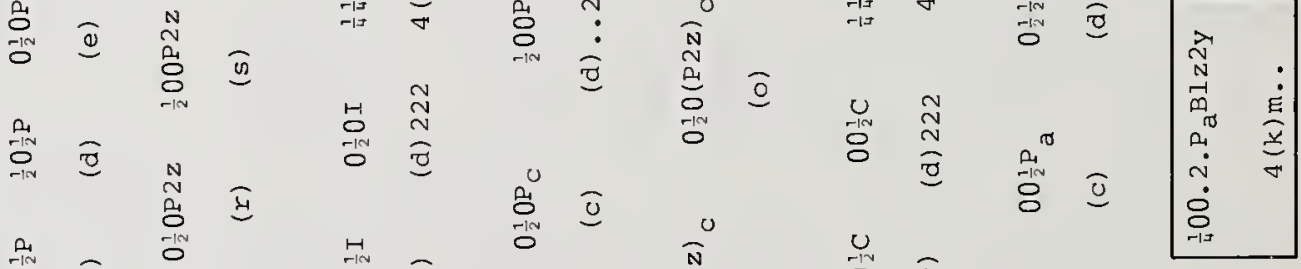

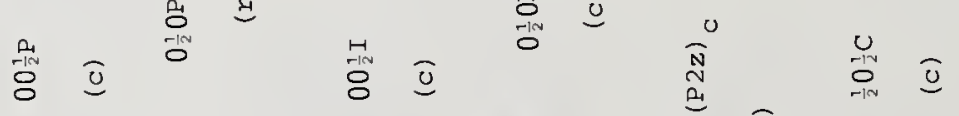

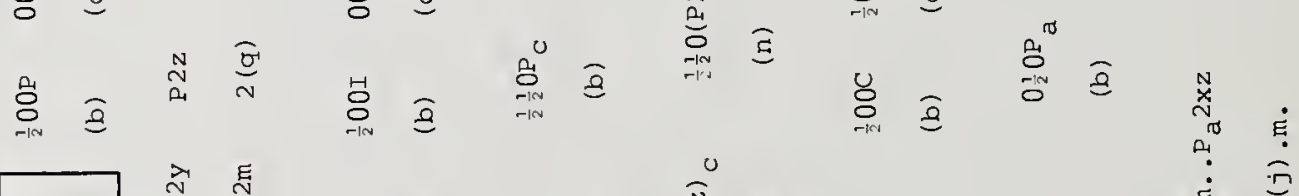

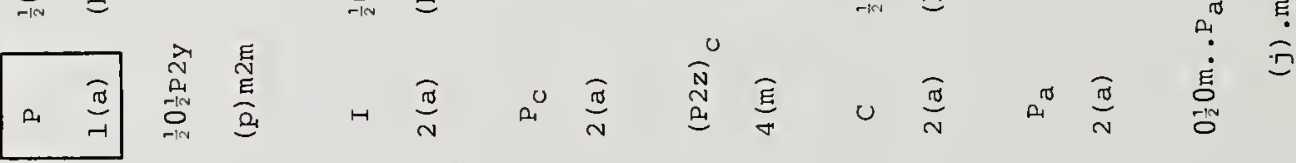

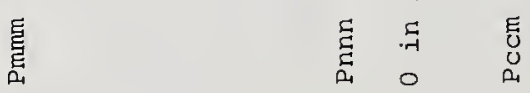

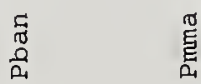




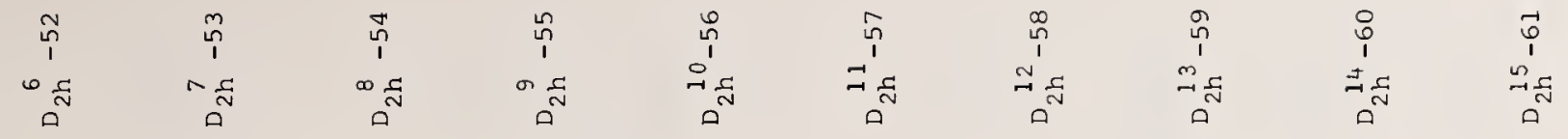
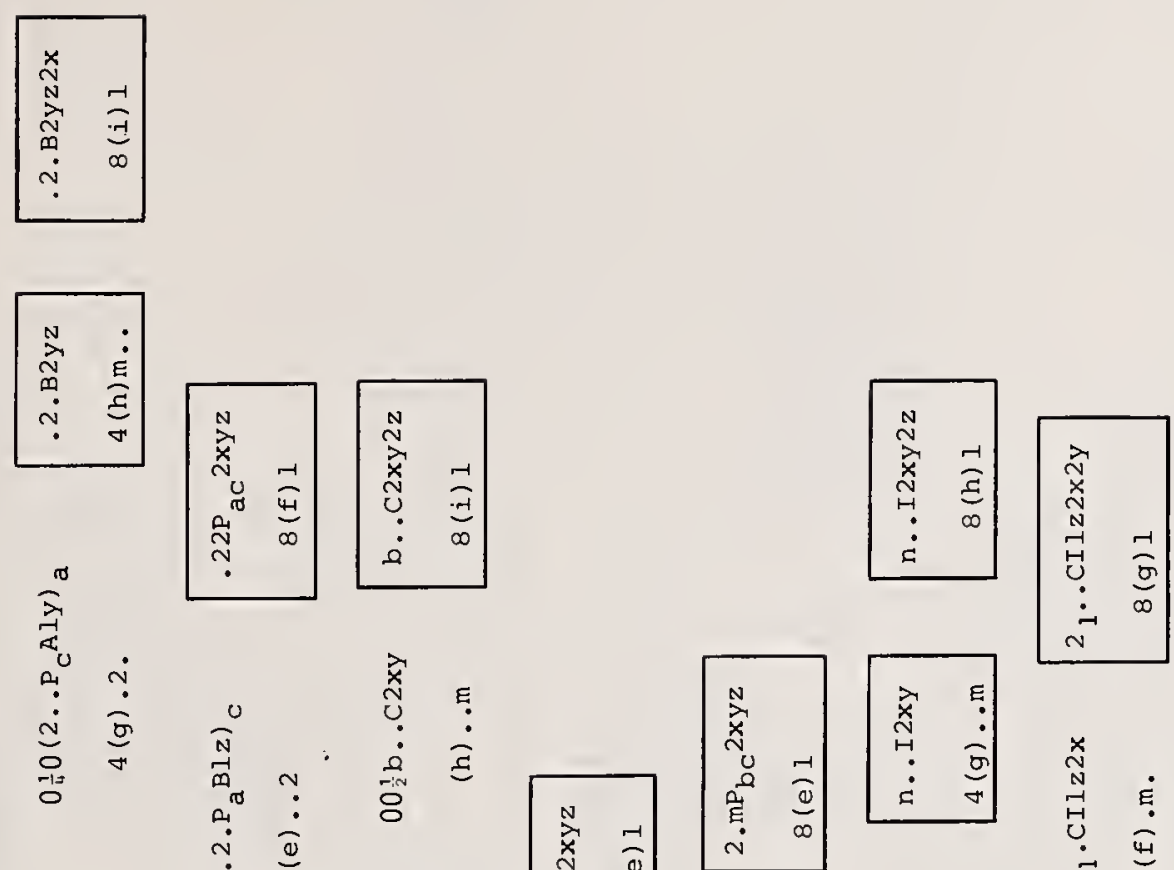

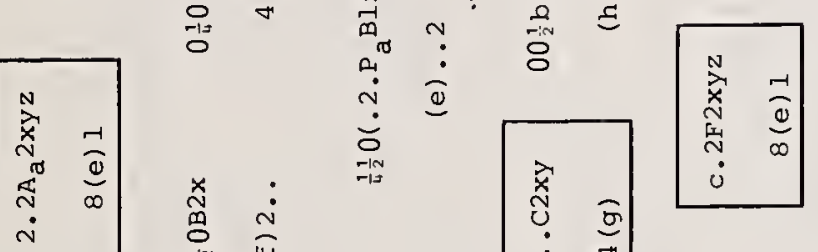
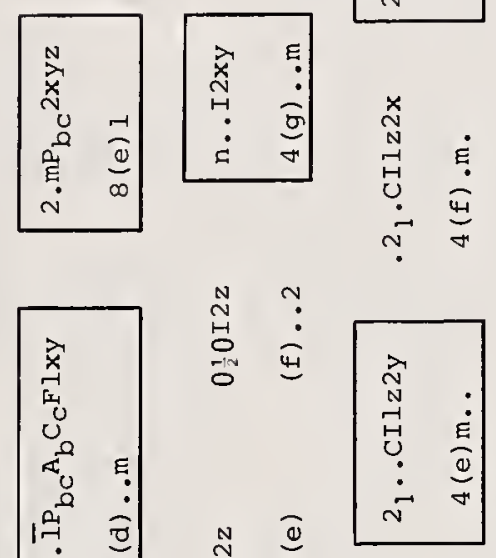

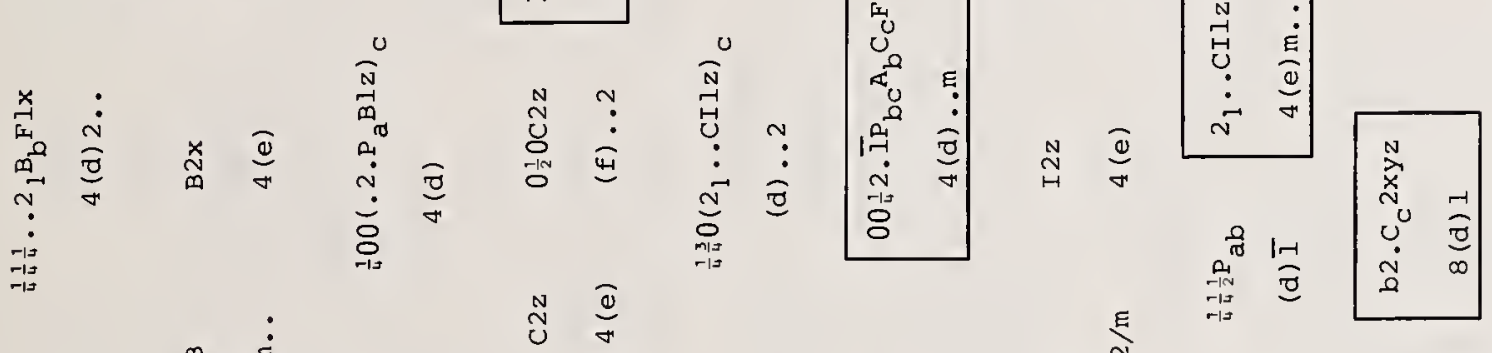

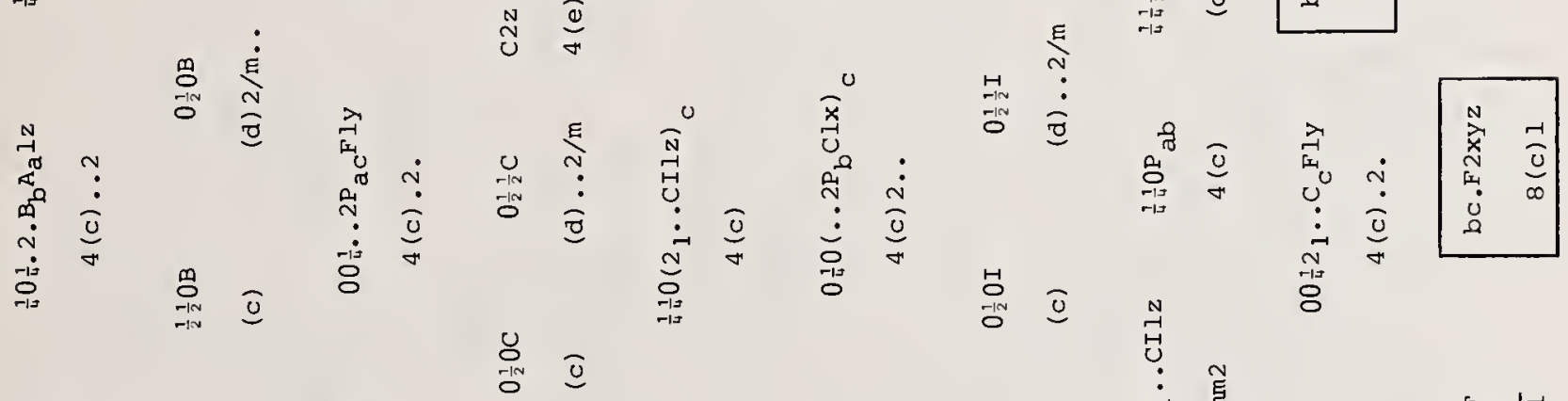

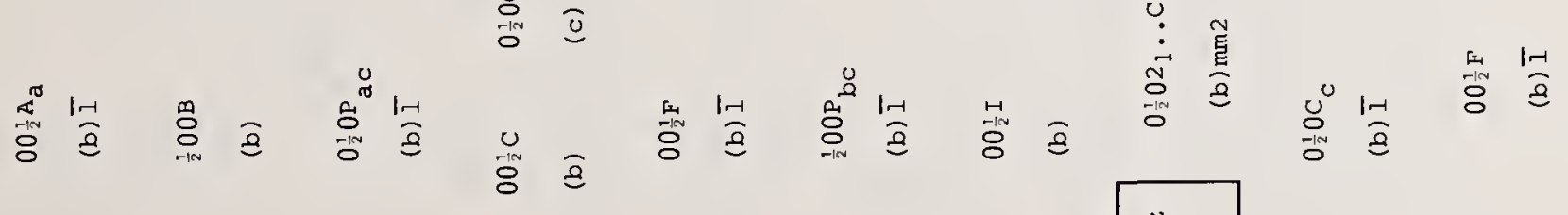

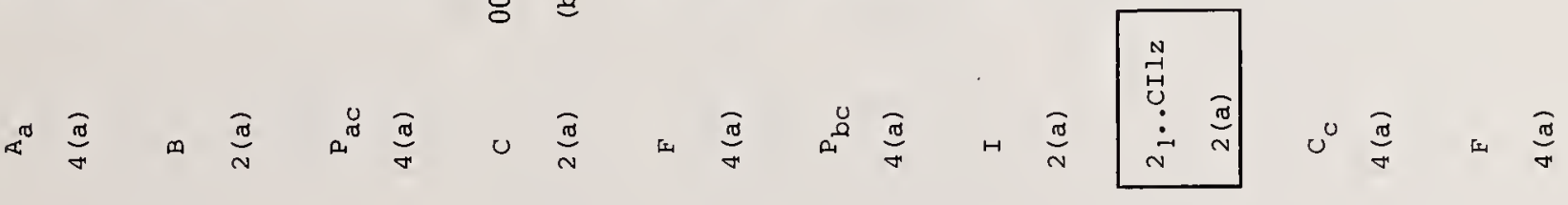

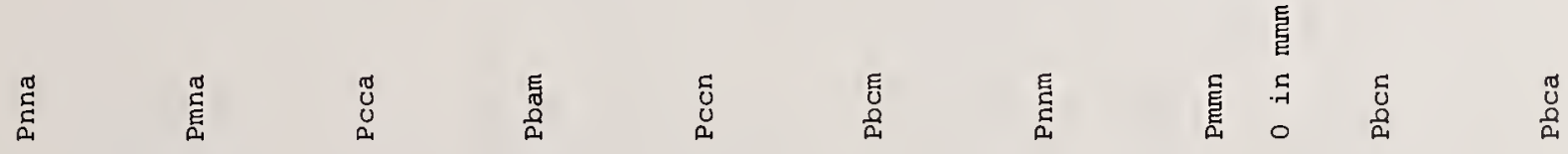




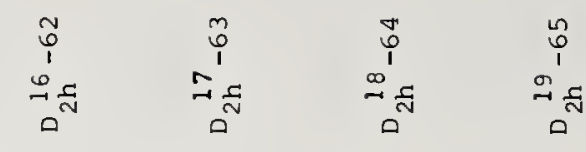

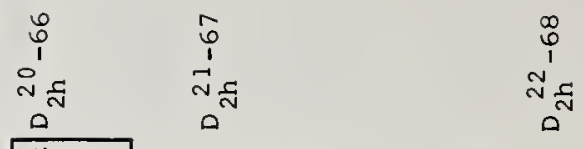

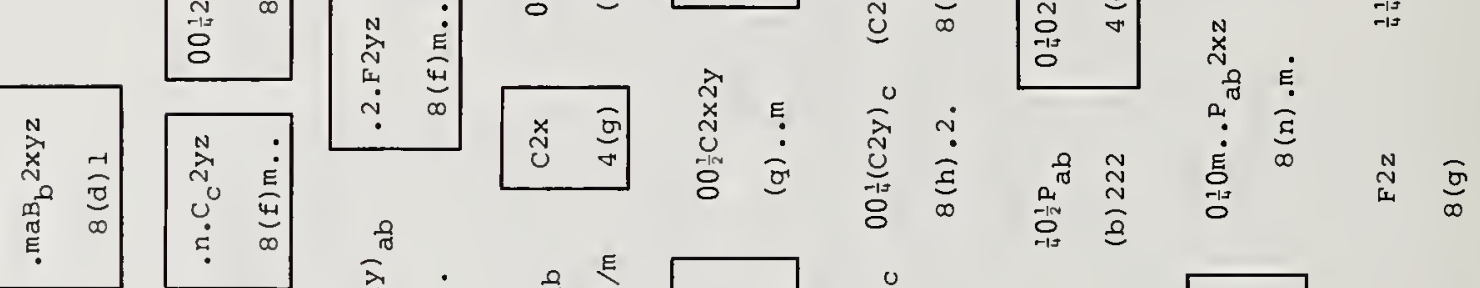

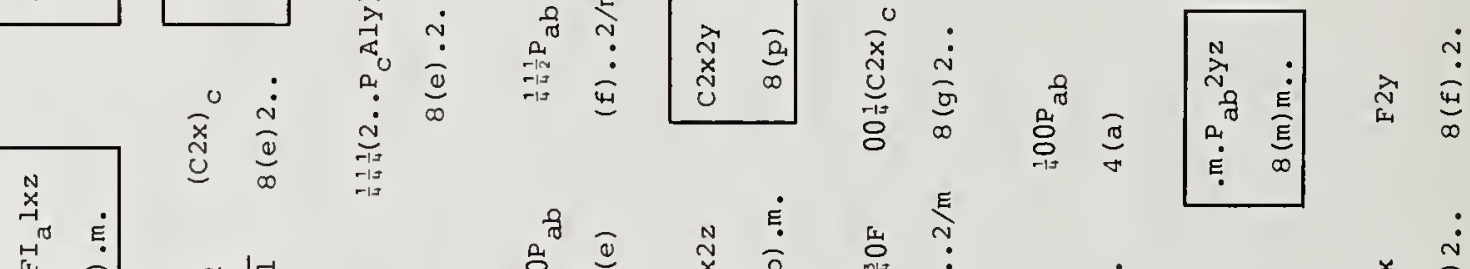

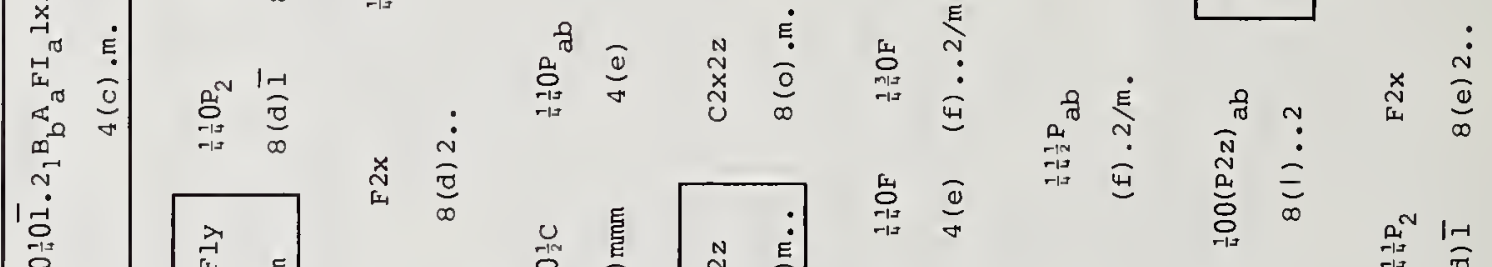

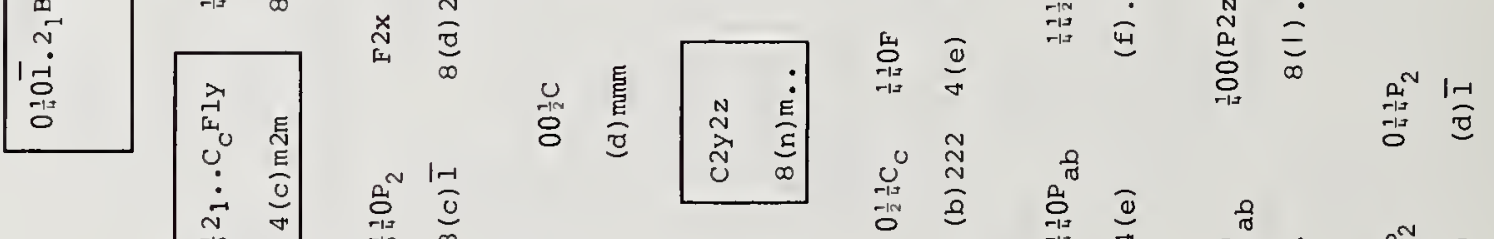

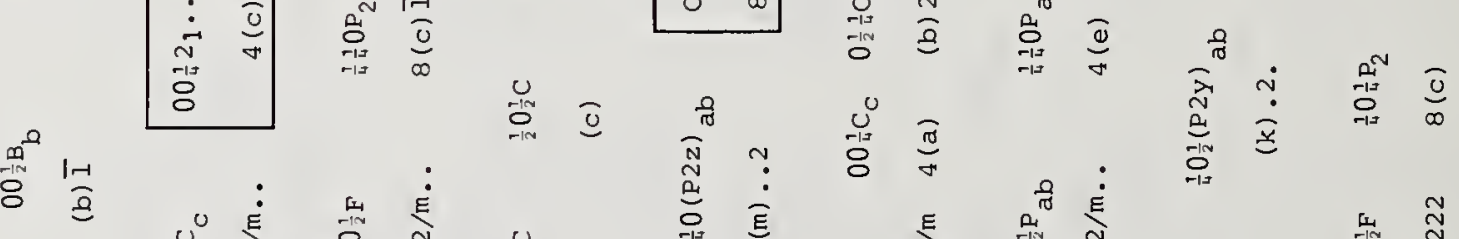

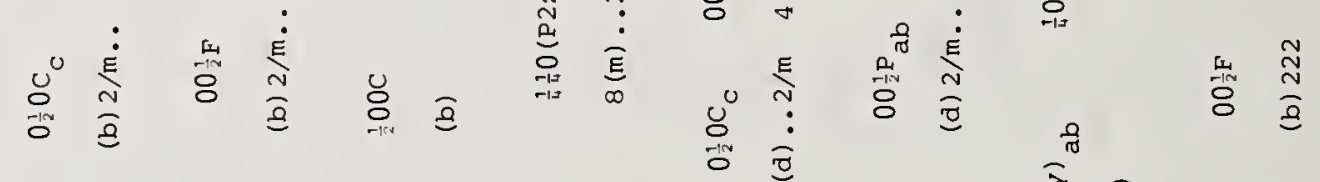

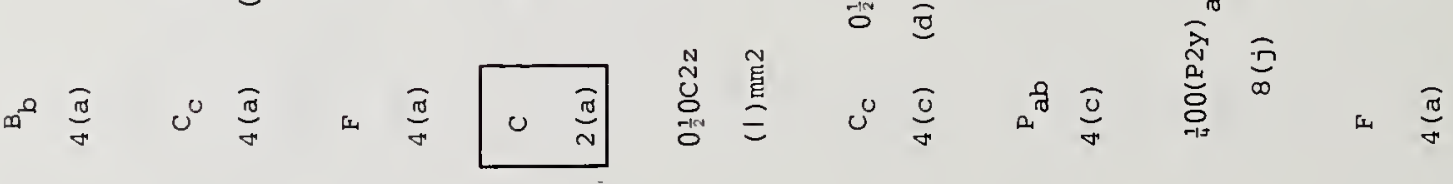

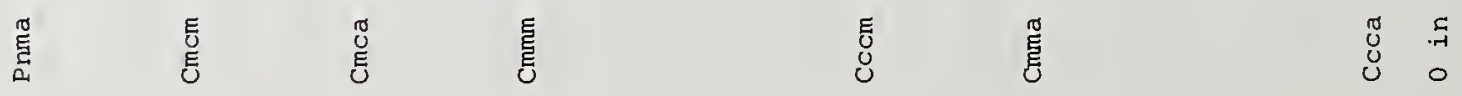




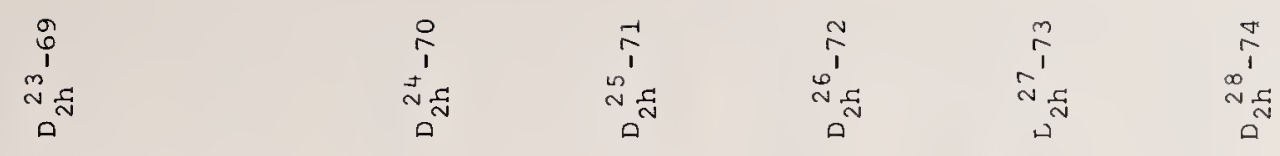

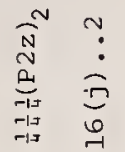

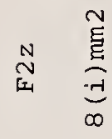
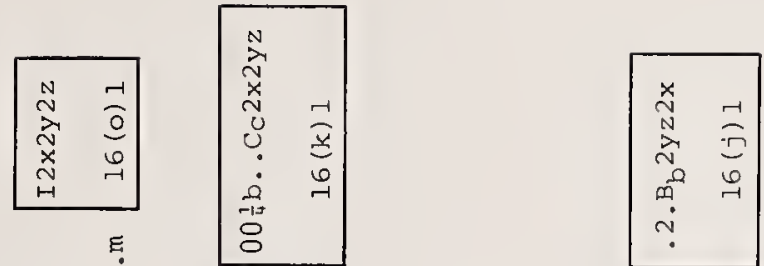

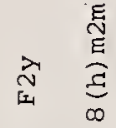

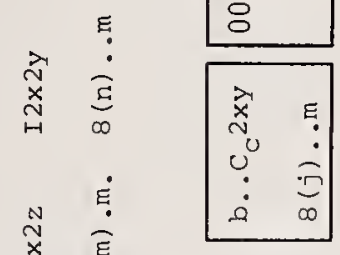

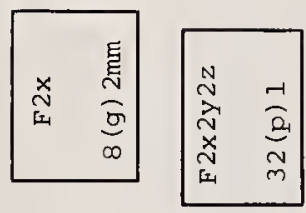

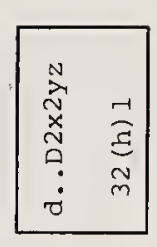

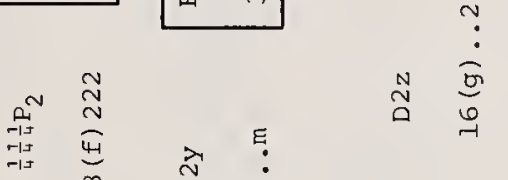

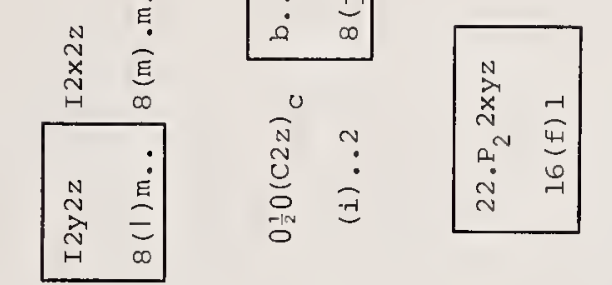

ㅊ

$\overbrace{\substack{\infty \\:}}^{-7}$

童

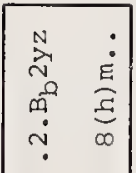

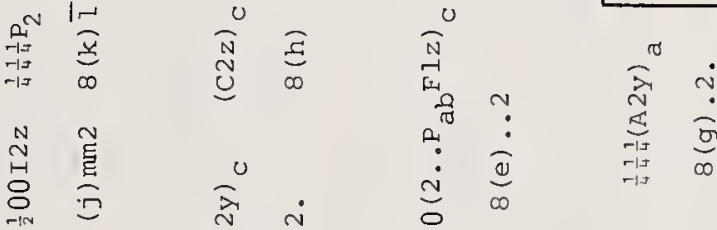

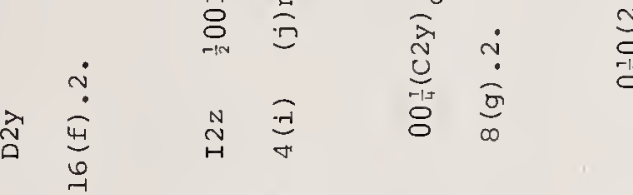

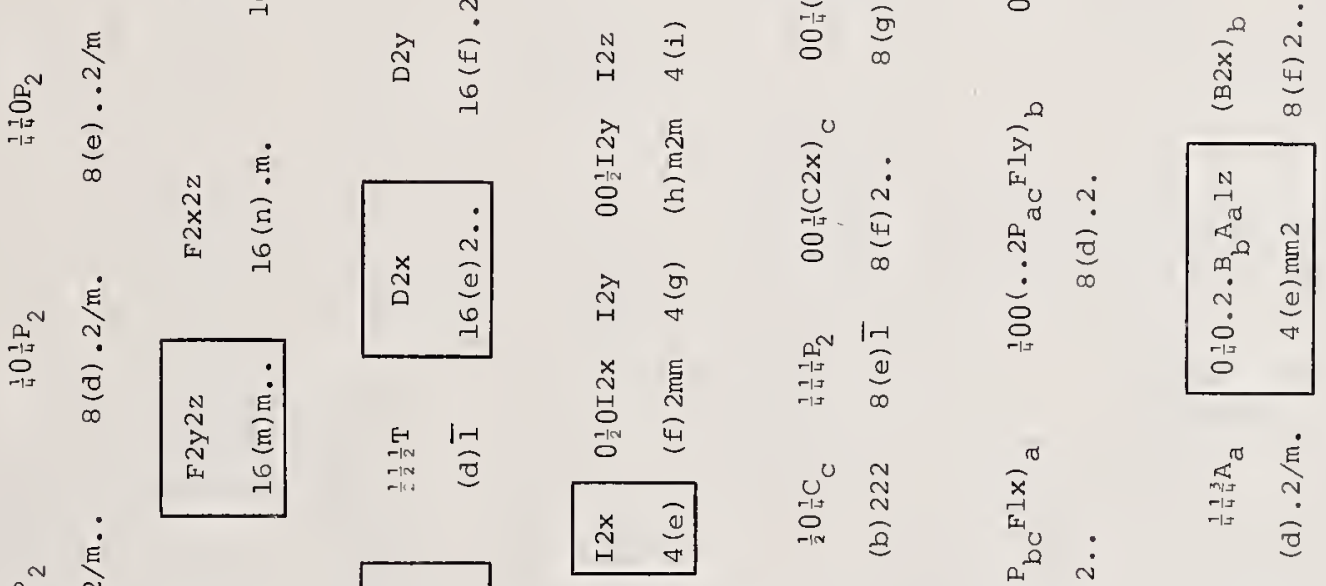

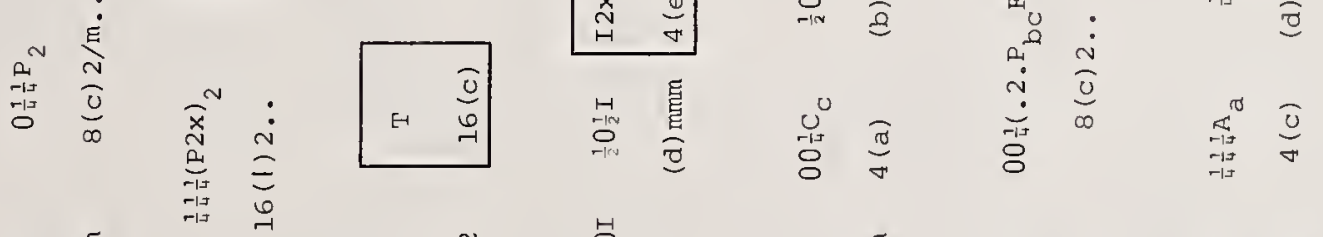

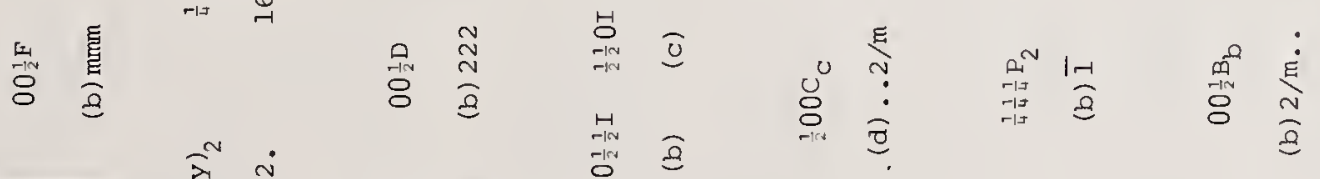

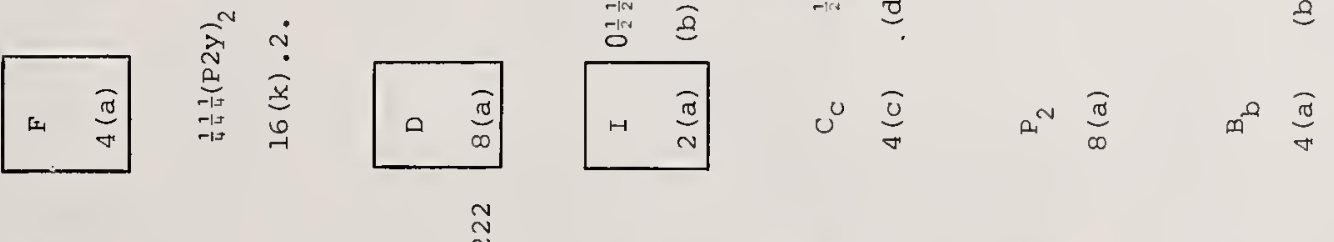

窰

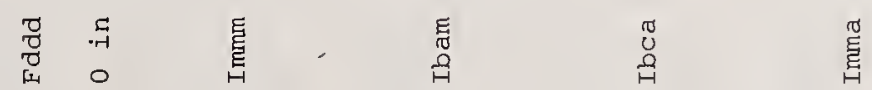




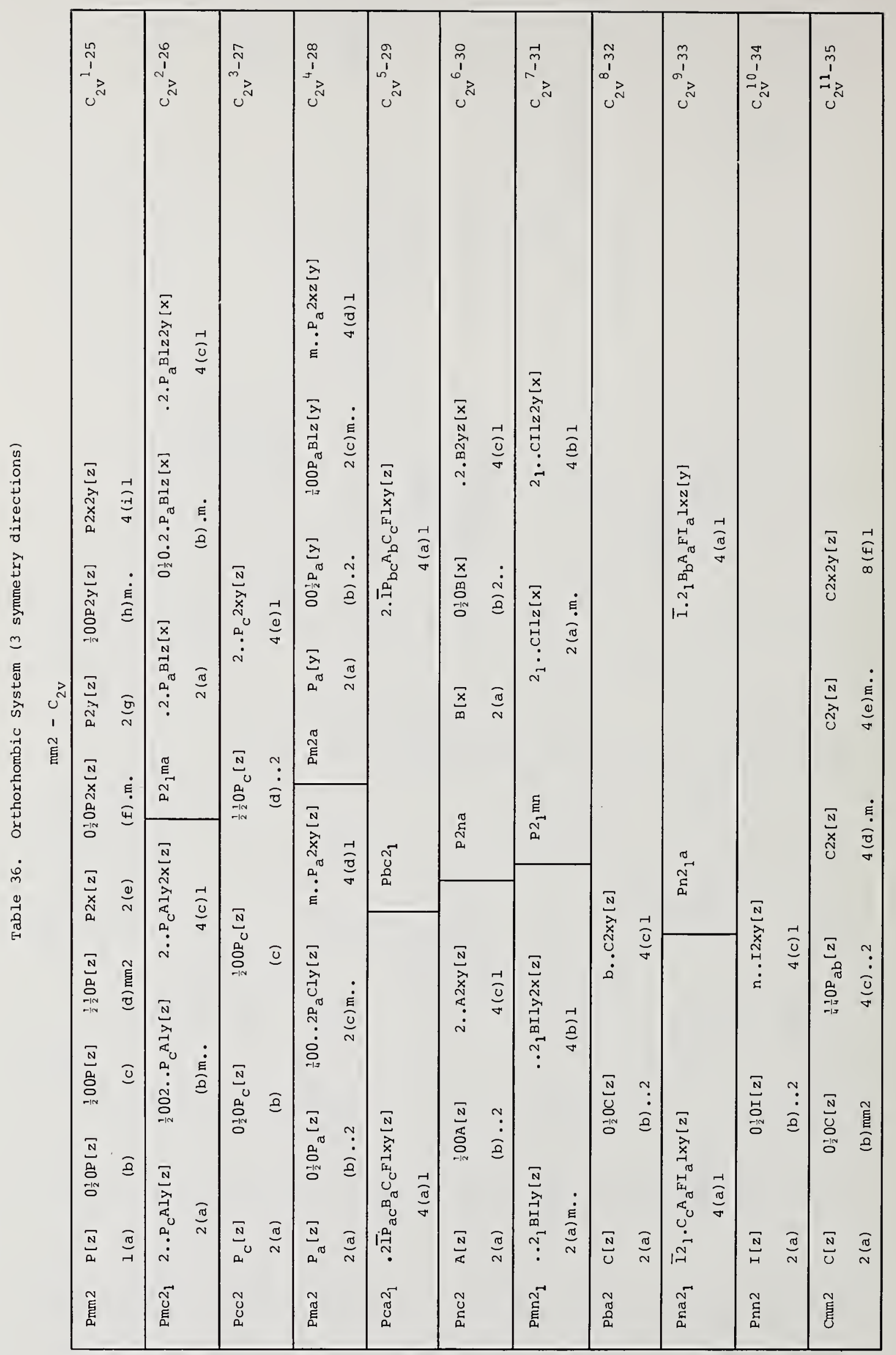




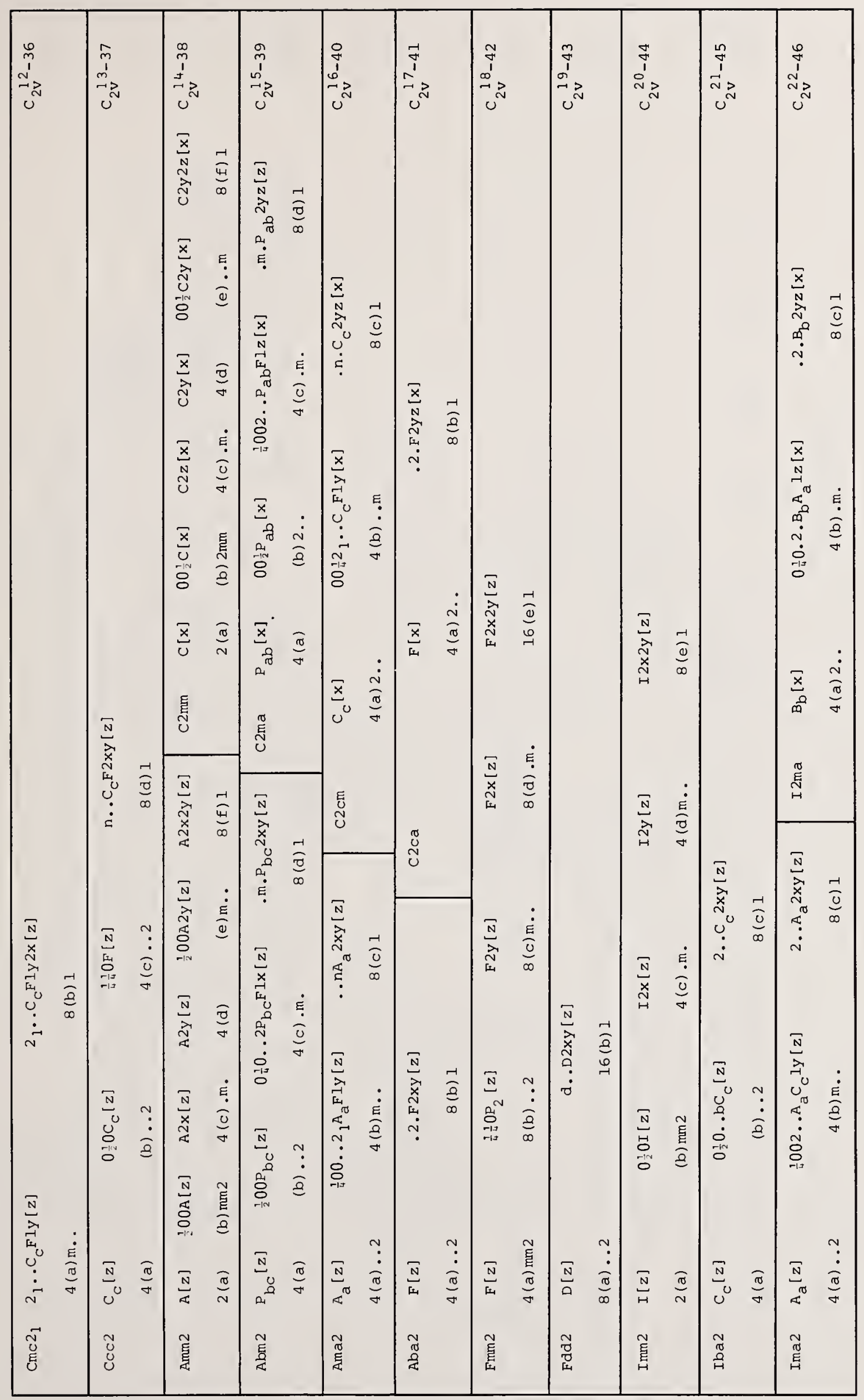




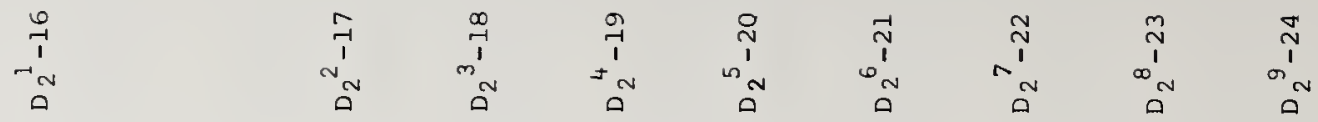

$\underset{\sim}{\stackrel{N}{N}}$

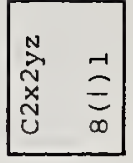

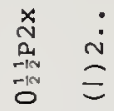

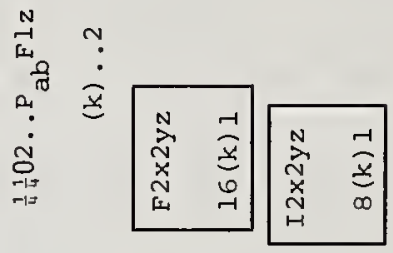

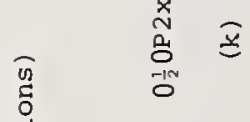

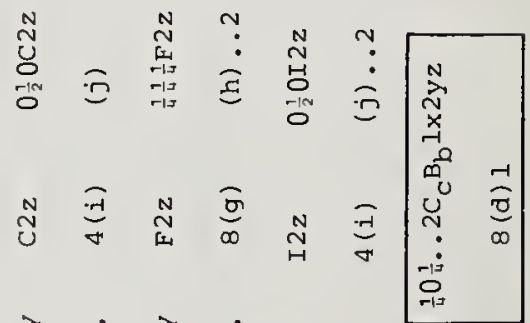

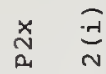

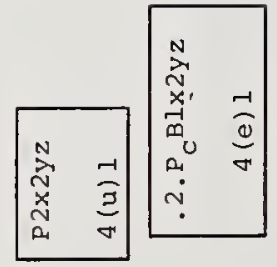

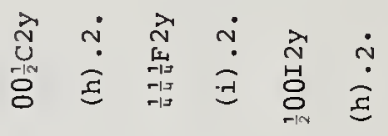

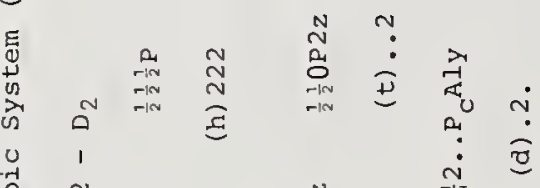

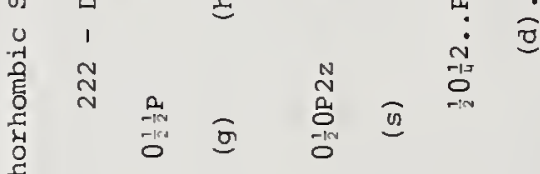

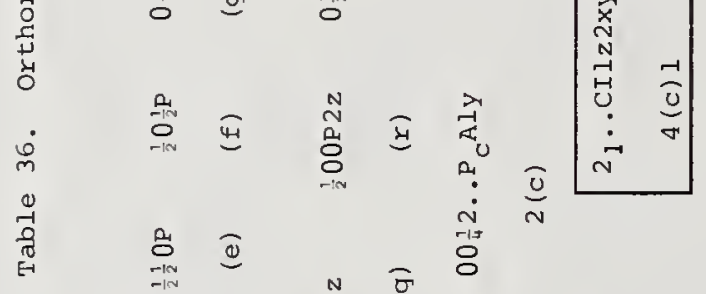

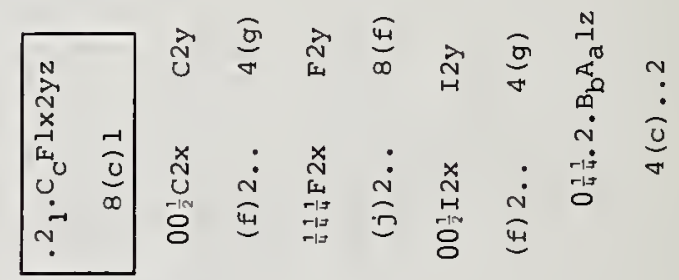

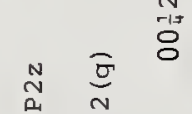

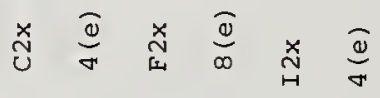

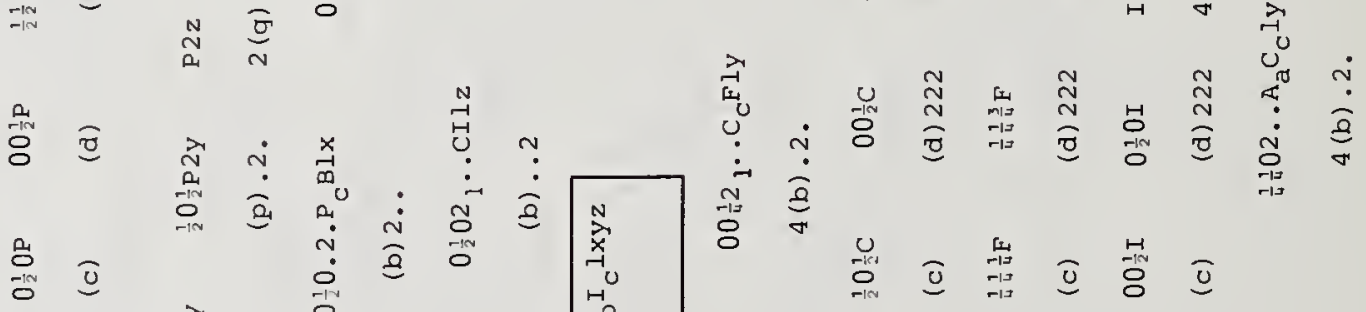

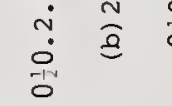

D.

$\underset{\sim}{N}$

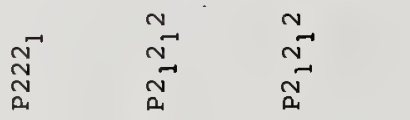

$\stackrel{\text { กิ }}{\text { ก }}$

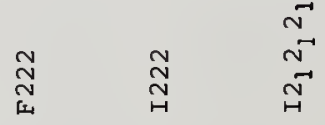




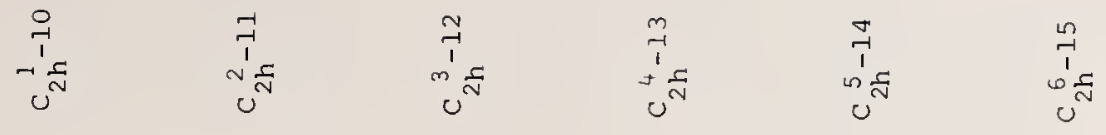

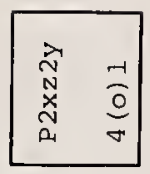

$\underset{\substack { N \\ \begin{subarray}{c}{\mathfrak{O} \\ 0{ N \\ \begin{subarray} { c } { \mathfrak { O } \\ 0 } }\end{subarray}}{\substack{0 \\ 0}}$

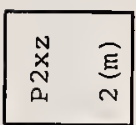

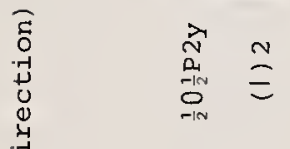

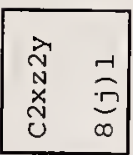

究

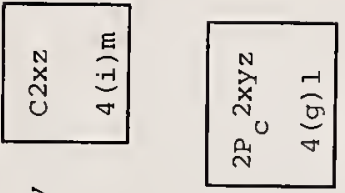

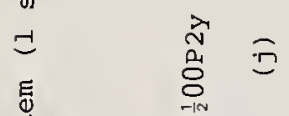

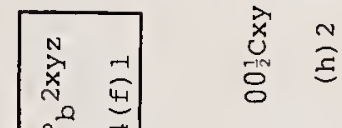

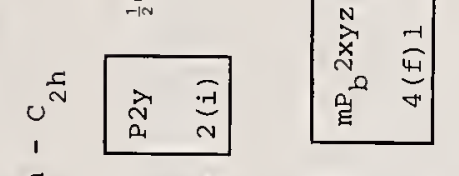

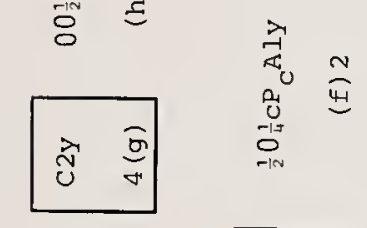

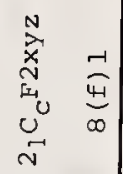

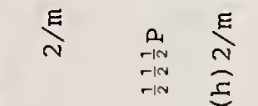

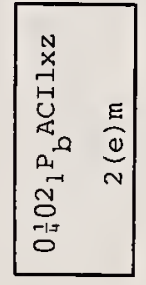

$\underset{\substack{-10 \\ \text {-ind }}}{\substack{0 \\ 0}}$

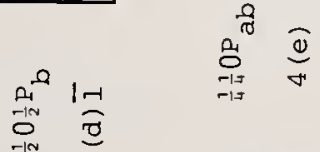

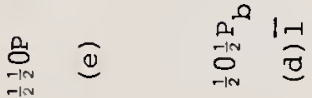

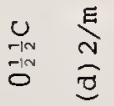

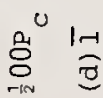

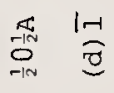

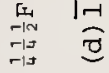

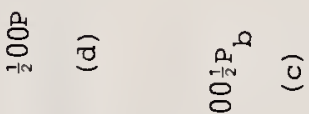

$\underset{\substack{0 \\ \text { oid }}}{0}$

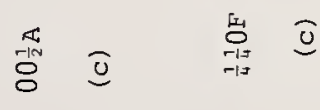

잉

$\underset{0}{0} 0$

a $0 \quad \delta_{-i N}^{0} \hat{a}$

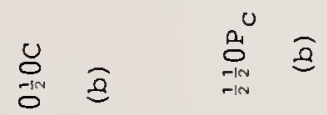

$\underset{-11}{\mathbb{8}}$

0
0
0
0

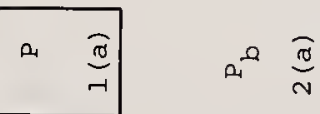

\begin{tabular}{ll|}
0 & $\pi$ \\
\hline & N \\
\hline
\end{tabular}

a.

$\varangle \frac{\pi}{N}$

$0 \underset{\sigma}{0}$

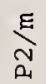

$\underset{\substack{n \\ \text { a }}}{E}$

$\underset{\text { ป }}{E}$

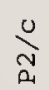

$\frac{\tilde{a}^{2}}{0}$

ปे 


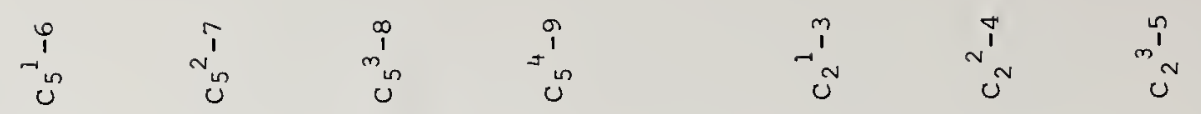

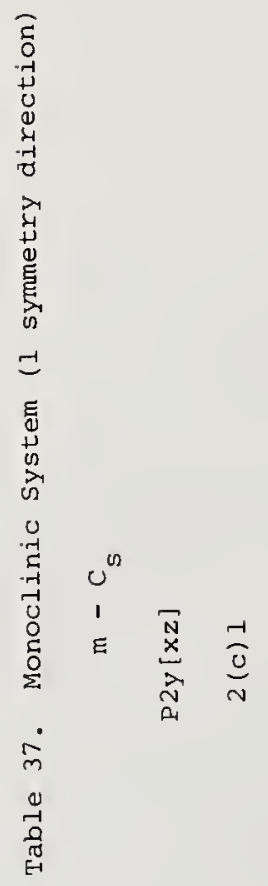

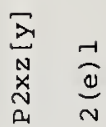

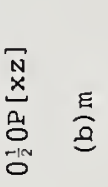

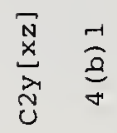

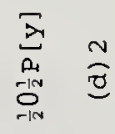

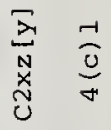

$\underset{-1 \times}{\mathbb{1}_{1}} 0$

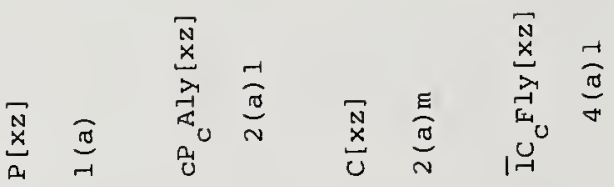

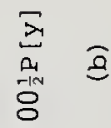

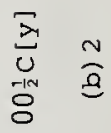

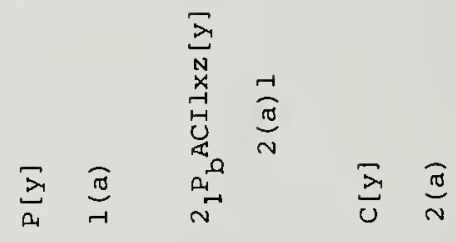

E

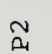

$\vec{n}$

ชี 


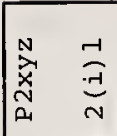

$$
\text { ב⿱ }
$$

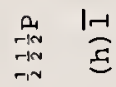

究

巳

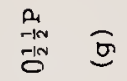

है

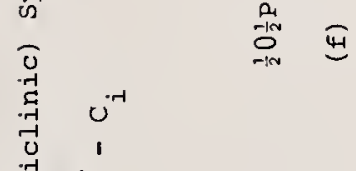

$\underset{\substack{-1 N \\ \text { IN }}}{\Delta}$

苟

$\stackrel{\infty}{m}$

拳

$\stackrel{9}{7}$

$\underset{\substack{0 \\ 0}}{\substack{1 \\ 0}}$

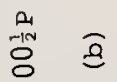

$2 \quad \stackrel{\pi}{-1}$

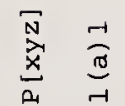

$\mid \vec{a}$

$\vec{A}$ 
APPENDIX

Subgroup Relations

Tables 39-43 


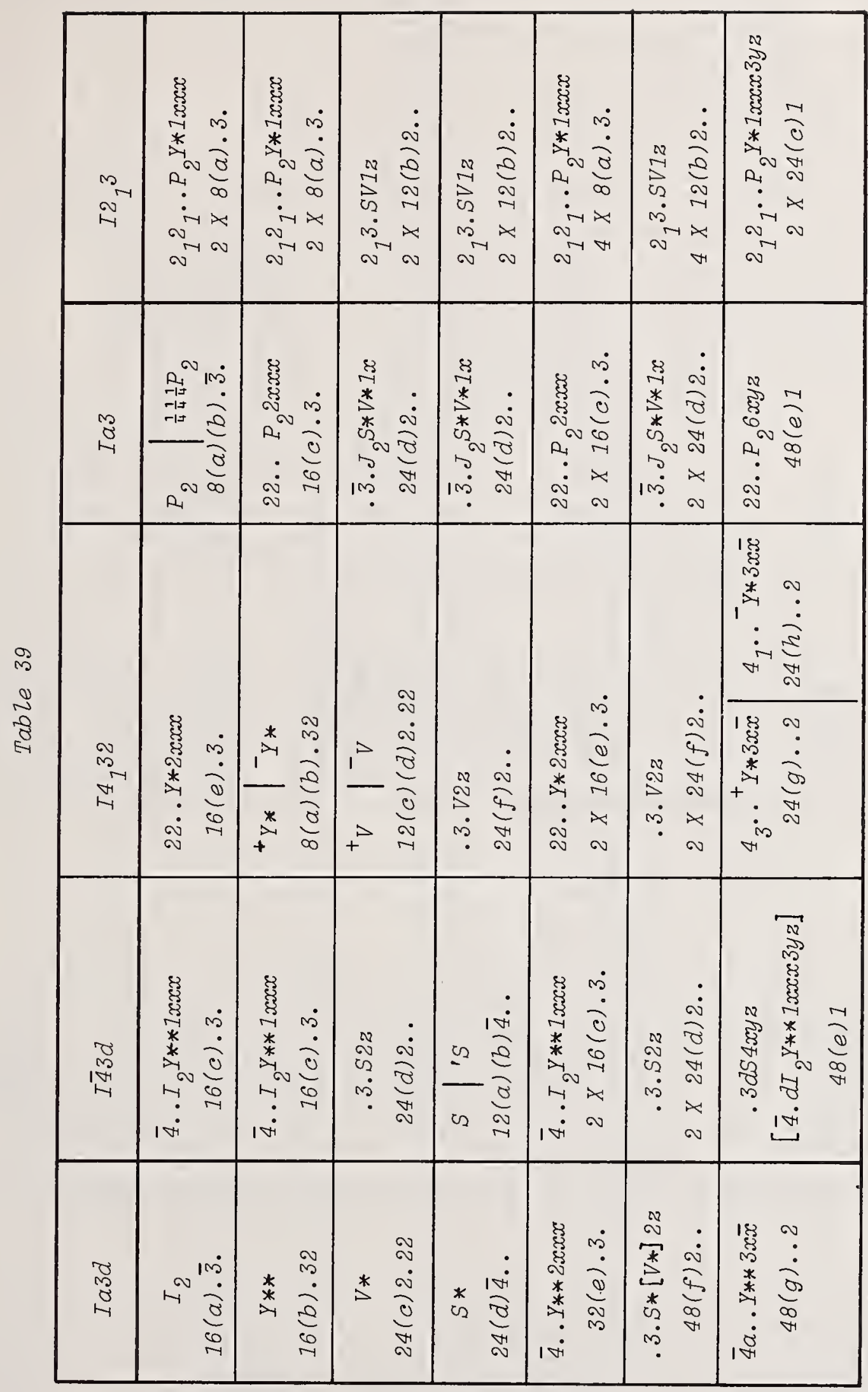




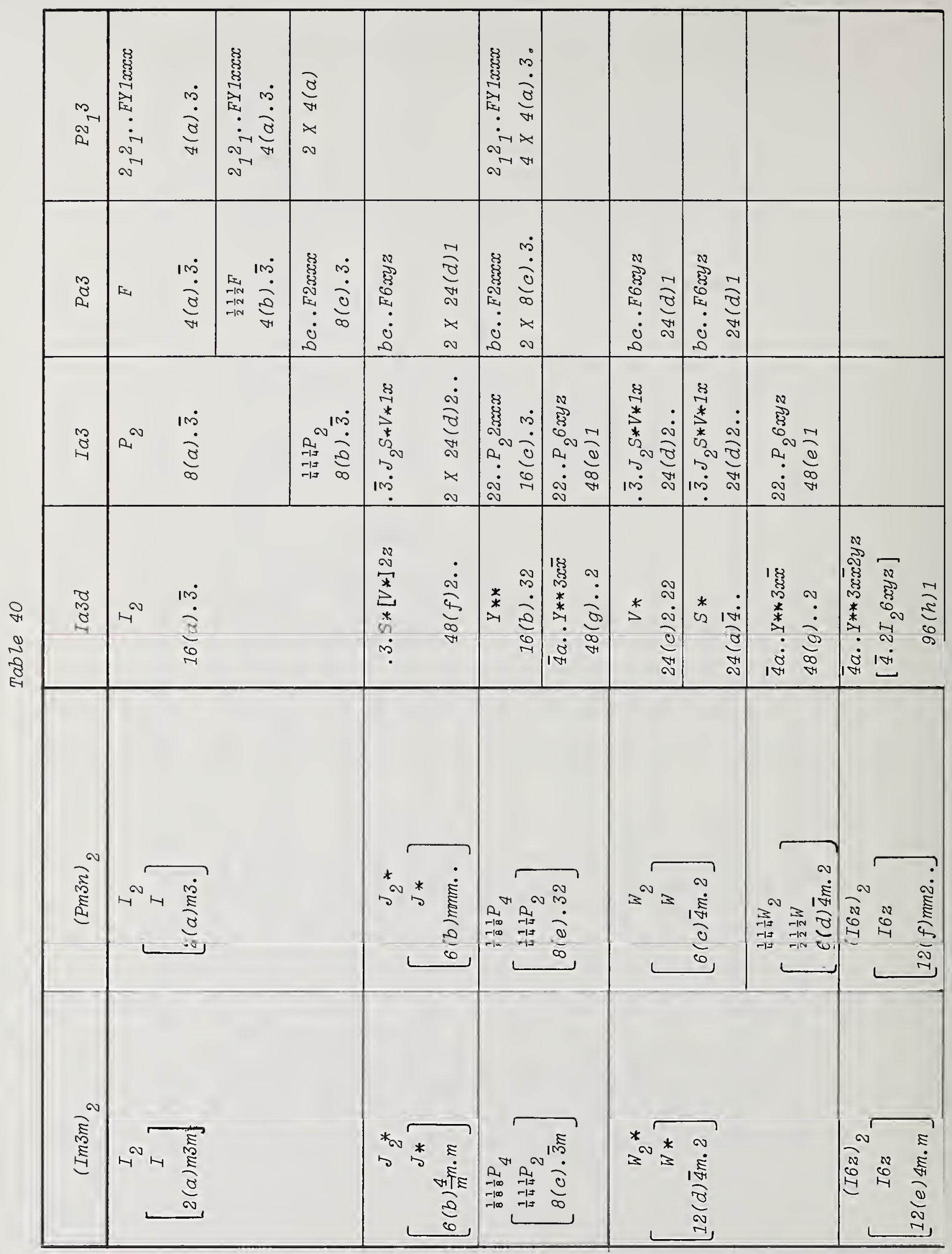




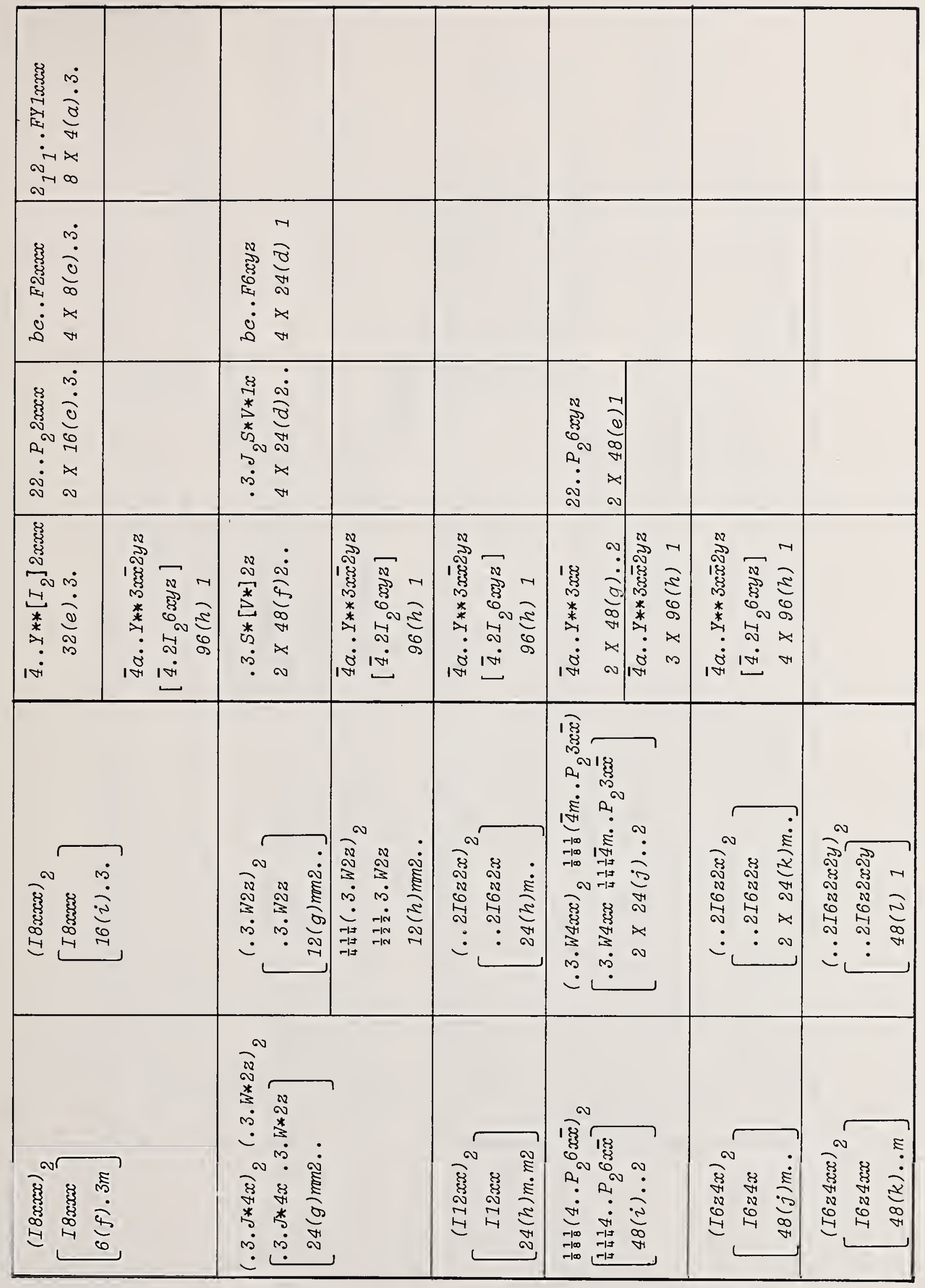




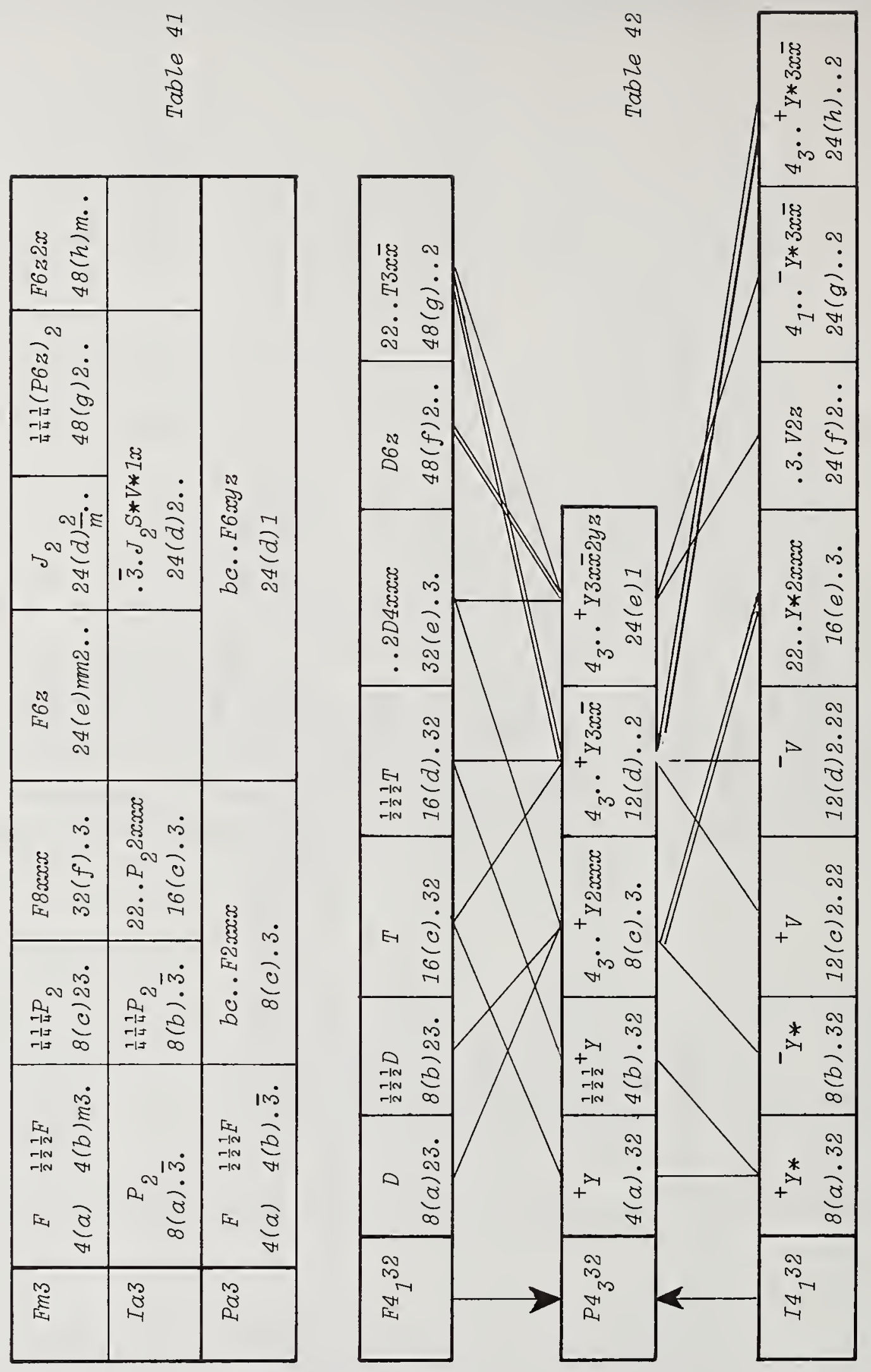




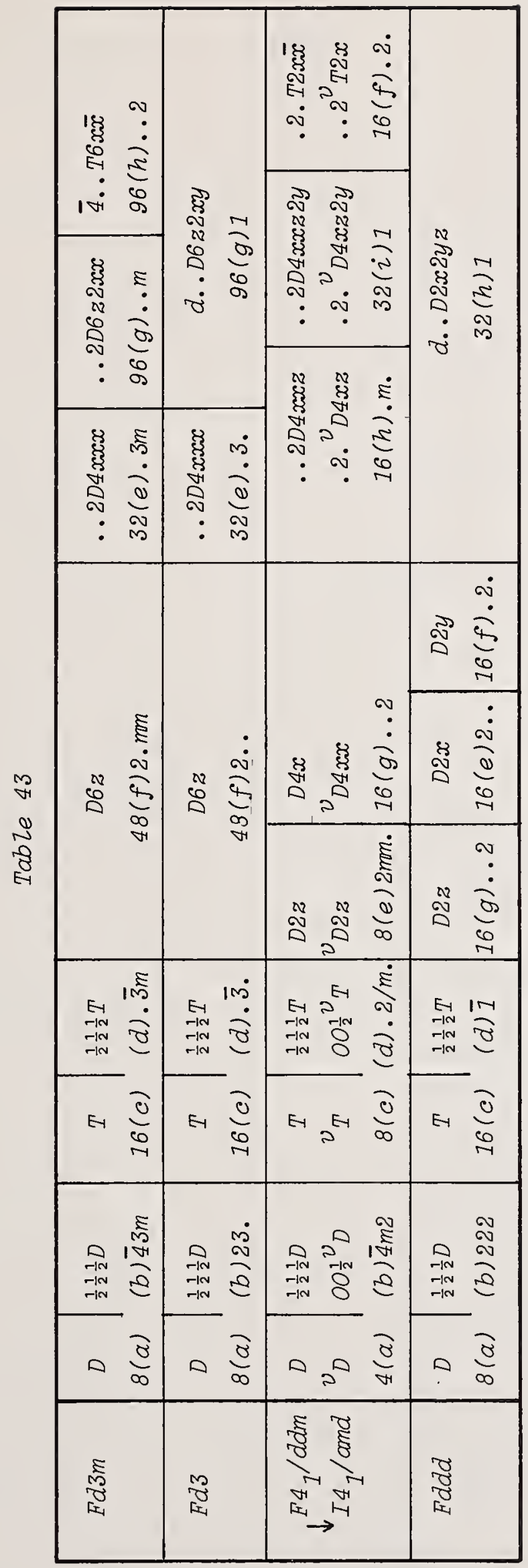



Anorthic system, 12

Asymmorphic, 23

Axis

a) ternating --, 3

co-ordinate --, 4

Neben --, 3

Zwischen --, 3

B1ickrichtungen, 3

Complex, 1

1attice -- (LC), 11, 85-131

Weissenberg complex, 18, 69-83

Configuration, 11

Co-ordinates

condensed description of, 8

Crystal

- class, 8

- form, 1, 6

- symmetry, 3

Degradation

cubic --, 9, 64

hexagonal --, 10,66

Distribution symmetry, 17

Dome (=dihedron), 7
Face complex, 1

Form, crystal form, 1, 4

General --, 4

special --, 4

Hauptgitter, 18

Hemisymmorphic, 23

High symmetry point (HSP), 3, 17

Isogonal polyhedron, 6

Lattice, 1

- complex, 1, 11

- symmetry, 3

Lattice complex (L.C.)

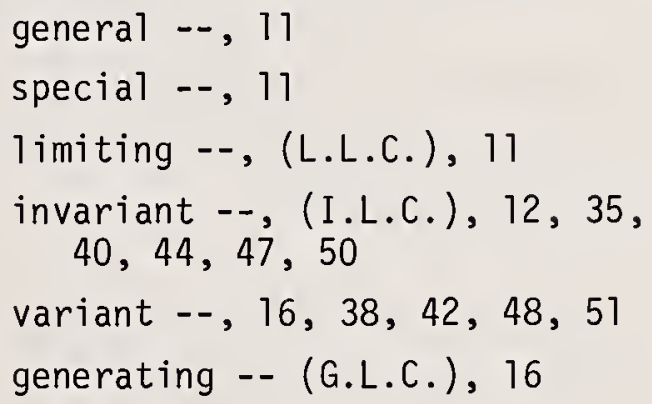

Limiting site set, 4

Multiplicity

- of a lattice complex,,$^{\circ} 11$

- of a site set, 8

Nodes, 1 
Parallelohedron (=pinacoid), 7

N-pointer, 3,6

iN-punkter

N- punktner, 3

Position, 2, 11

point --, 2, 11

Prime

prime sign on Wyckoff 1etter, 19

Punktlage, 2

References, 26

Representation

- of a lattice complex, 11

standard --, 11

alternate --, 12

shifted --, 14

congruent --, 14

enantiomorphous --, 14

Rhombicuboctahedron, 8

Shift vector, 14

Site

- set, $3,6,29,55$

- symmetry, 3, 11, 16, 62

general site set, 4

special site set, 4

generating site set, 5
Space groups, 133-167

characteristic -- of a L.C., 11,12

Sphenoid (=dihedron), 7

Splitting, 5, 54

partial --, 5

complete --, 5

Subgroup relations

- in point groups, 9, 34

- in space groups, 23, 169-173

Symbols

Hermann-Mauguin -- , 3

oriented --, 4

Symmetry

- direction, 3

- element, 3

- operation, 17

Symmorphic, 23

Synonyms of G.L.C., 17

Transformation of co-ordinates,

4 - and 3 - index symbols, 9

Triclinic (=anorthic) system, 12

Truncations, 8

Weber four-index symbols, 9

Snub cube, 8 
p. 17, Sn. 6.4, line $\overline{4}$ : in front of space group insert characteristic

p.133, $\operatorname{Im} 3 \mathrm{~m}\left(\mathrm{O}_{\mathrm{h}}{ }^{9}\right)$, second line of entry, in $6(\mathrm{c}) \cdot \overline{3} \mathrm{~m}$ : replace 6 with 8

$\mathrm{p} .138, \mathrm{P}_{3} / \mathrm{mcm}\left(\mathrm{D}_{6 \mathrm{~h}}{ }^{3}\right)$, position $4(\mathrm{e}) 3 . \mathrm{m}$ is correctly given. [Note erratum in I.T. (1965, p.303): instead of 4 (e) 6 read 4(3) $3 \mathrm{~m}$ ]

p.150, $\mathrm{P} 4 / \mathrm{nmm}\left(\mathrm{D}_{4 \mathrm{~h}}{ }^{7}\right)$, first line of entry, above $(\mathrm{h}) \ldots 2$ : replace $0 \frac{1}{2} 0$ with $00 \frac{1}{2}$

p. 150, $\mathrm{P} 4 / \mathrm{ncc}\left(\mathrm{D}_{4 \mathrm{~h}}{ }^{8}\right)$, second line of entry, after $4(\mathrm{c})$ : replace $\overline{4}$.. with 4 ..

p.154, $\mathrm{P} 4 \mathrm{bm}\left(\mathrm{C}_{4 \mathrm{v}}{ }^{2}\right)$, first line of entry, above $4(\mathrm{c}) \ldots \mathrm{m}$ : replace $0 \frac{1}{2} 0 . \mathrm{b} \cdot \mathrm{C} 2 \mathrm{xx}$ with $0 \frac{1}{2} 0 \cdot \mathrm{b} \cdot \mathrm{C} 2 \mathrm{xx}[\mathrm{z}]$

P.155, $\mathrm{P}_{2}{ }_{2}{ }_{1} 2\left(\mathrm{D}_{4}{ }^{6}\right)$, second line of entry:

replace $4(\mathrm{c})$ with $4(\mathrm{c}) 2$.. replace (d) $2 \ldots$ with 4 (d) 2 ..

p.158, Pban $\left(D_{2 h}{ }^{4}\right)$, under Pban add choice of origin: 0 in 222

p.165, C2/c $\left(\mathrm{C}_{2 \mathrm{~h}}{ }^{6}\right)$, second line of entry:

$$
\begin{array}{lll}
\text { replace (b) with } & \text { (b)i } 1 \\
\text { replace } & \text { (c) with } & \text { (c) }
\end{array}
$$

-Remark.- Some of the conventional position letters used by Wyckoff (1930) have occasionally been interchanged in Vol. I of the I.T. (1952 and reprintings). The original Wyckoff letters have been used in the present lattice-complex tables. Agreement with the I.T. can be achieved by means of the following changes.

p.105, under $\mathrm{P}$, at $\mathrm{P}_{c}, 2, \overline{4} 2 \mathrm{~m}, \mathrm{P}_{4} / \mathrm{mcm}$ :

$$
\frac{\text { replace }}{\text { replace (a) with (b) with }} \text { (d) }
$$

[Note that (b) should have read (c)]

$\mathrm{p} .105$, under $\mathrm{P}$, at $\mathrm{P}_{c}, 2, \mathrm{~m} . \mathrm{mm}, \mathrm{P}_{2} / \mathrm{mcm}$ :

$$
\begin{array}{lll}
\text { replace } & \text { (b) with (a) } \\
\text { replace } & \text { (d) with } & \text { (c) }
\end{array}
$$

p.108, under $I$, at $F_{c}, 8, \overline{4} \ldots, I^{1}{ }_{1} /$ acd:

on the next line, at $2.22,14 / \mathrm{replace}$ : replace (a) with (b)

$\mathrm{p} .150, \mathrm{P}_{2} / \mathrm{mcm}\left(\mathrm{D}_{4 \mathrm{~h}}{ }^{10}\right)$ : interchange (a) and (b); 1ikewise (c) and (d)

$\mathrm{p} .151, \mathrm{I}_{1} / \mathrm{acd}\left(\mathrm{D}_{4 \mathrm{~h}}{ }^{20}\right)$ : interchange (a) and (b)

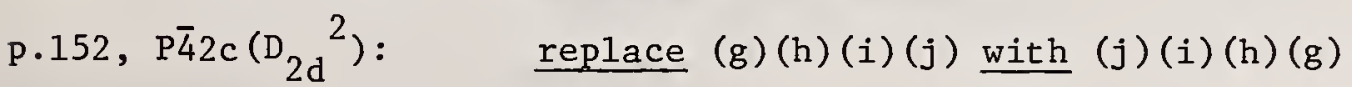

Wyckoff, R.W.G. (1930). The analytical expression of the results of the theory of space groups. Second edition. Carnegie Institution of Washington. 
FORM NBS-114A (1.71)

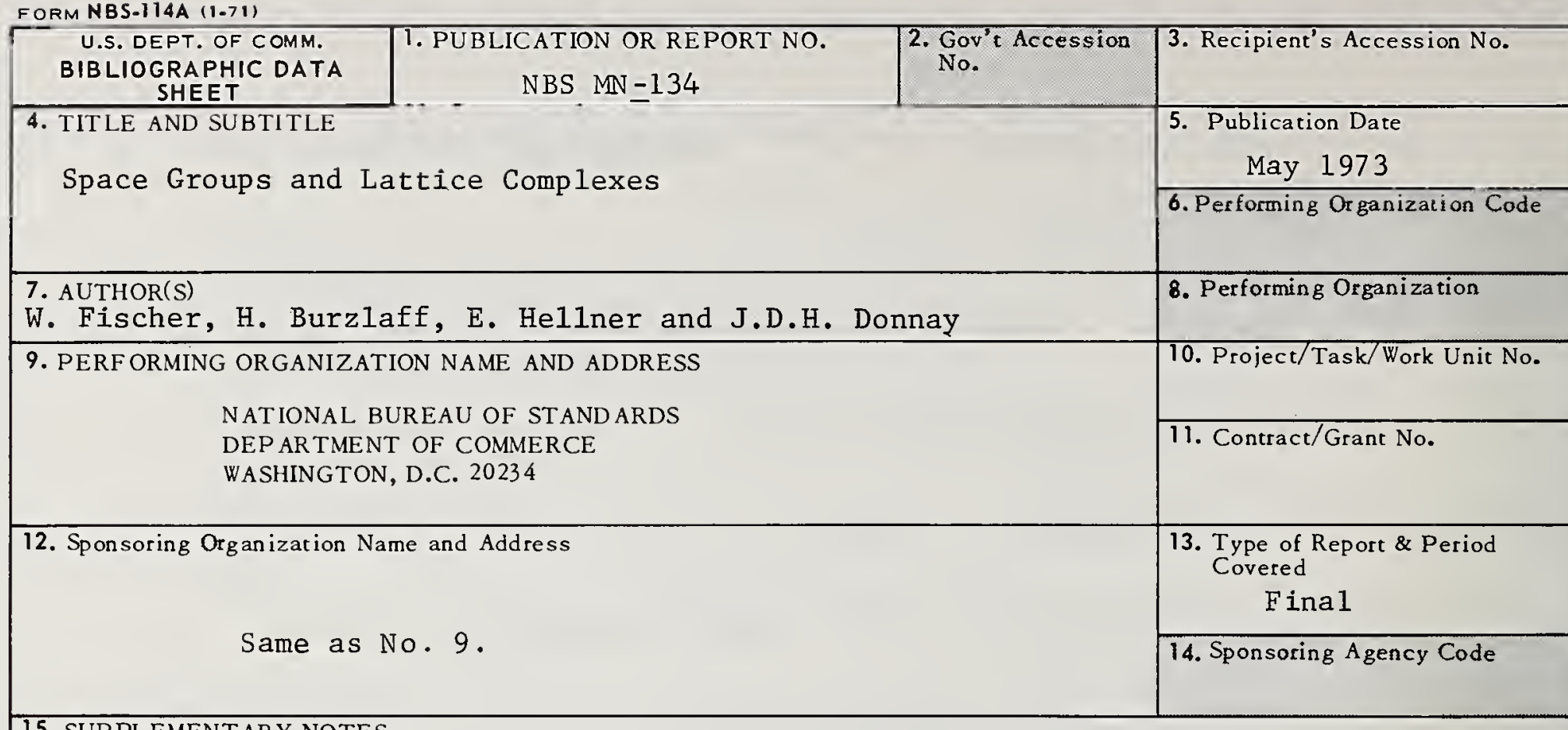

15. SUPPLEMENTARY NOTES

16. ABSTRACT ( $A$ 200-word or less factual summary of most significant information. If document includes a significant bibliography or literature survey, mention it here.)

The lattice complex is to the space group what the site set is to the point groupan assemblage of symmetry-related equivalent points. The symbolism introduced by Carl Hermann has been revised and extended. A total of 402 lattice complexes are derived from 67 Weissenberg complexes. The Tables list site sets and lattice complexes in standard and alternate representations. They answer the following questions: What are the co-ordinates of the points in a given lattice complex? In which space groups can a given lattice complex occur? What are the lattice complexes that can occur in a given space group? The higher the symmetry of the crystal structures is, the more useful the lattice-complex approach should be on the road to the ultimate goal of their classification.

17. KEY WORDS (Alphabetical order, separated by semicolons) Crystallography; crystal point groups; crystal structure; lattice complexes; site sets; space groups.

\section{AVAILABILITY STATEMENT}

$X$ UNL IMITED.

FOR OFFICIAL DISTRIBUTION. DO NOT RELEASE TO NTIS.

\begin{tabular}{|l|c|}
\hline $\begin{array}{c}\text { 19. SECURITY CLASS } \\
\text { (THIS REPORT) }\end{array}$ & 21. NO. OF PAGES \\
UNCL ASSIFIED & 184 \\
\hline $\begin{array}{l}\text { 20. SECUR ITY CLASS } \\
\text { (THIS PAGE) }\end{array}$ & 22. Price \\
UNCL ASSIFIED & $\$ 4.10$ \\
\hline
\end{tabular}

USCOMM-DC $66244-P 71$ 


(i)

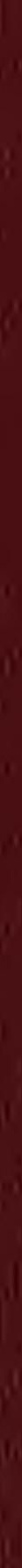

जlu 